\title{
CFD as Applied to the Design Of Short Takeoff and Landing Vehicles Using Circulation Control
}

\author{
A Thesis \\ Presented to the Faculty of \\ California Polytechnic State University, San Luis Obispo
}

In Partial Fulfillment of the

Requirements for the Degree of

Master of Science in

Aerospace Engineering

By

Tyler Matthew Ball

June 2008 
(0) 2008

Tyler Matthew Ball

ALL RIGHTS RESERVED 


\section{Approval Page}

Title: CFD as Applied to the Design of Short Takeoff and Landing Vehicles Using Circulation Control

Author: Tyler Matthew Ball

Date Submitted: June 2008

Dr. David D. Marshall

Advisor/Committee Chair

Signature

Dr. Jin Tso

Committee Member

Signature

Dr. Robert A. McDonald

Committee Member

Signature

Dr. Kim Shollenberger

Committee Member

Signature 


\section{Abstract \\ Title: CFD as Applied to the Design of Short Takeoff and Landing Vehicles Using Circulation Control}

\section{Author: Tyler Matthew Ball}

The ability to predict the distance required for an aircraft to takeoff is an essential component of aircraft design. It involves aspects related to each of the major aircraft systems: aerodynamics, propulsion, configuration, structures, and stability and control. For an aircraft designed for short takeoffs and landings (STOL), designing the aircraft to provide a short takeoff distance, or more precisely the balanced field length (BFL), often leads to the use of a powered lift technique such as circulation control (CC). Although $\mathrm{CC}$ has been around for many years, it has never been used on a production aircraft. This is in part due to the lack of knowledge as to how well CC can actually perform as a high lift device. This research provides a solution to this problem. By utilizing high fidelity computational fluid dynamics (CFD) aerodynamic data, a four-dimensional design space which was populated and modeled using a Monte Carlo approach, and a Gaussian Processes regression technique, an effective aerodynamic model for $\mathrm{CC}$ was produced which was then used in a BFL simulation. Three separate models were created of increasing quality which were then used in the BFL performance calculations. A comprehensive gridding methodology was provided as well as computational and grid dependence error analysis. Specific consideration was given to the effect of resolving the turbulent boundary layer in both the gridding and solving processes. Finally, additional turbulence model validation work was performed, both to match previously performed experimental data and to provide a comparison of different models' abilities to predict separation. 


\section{Acknowledgments}

There are a number of people which need to be mentioned for their support throughout my time spent here at Cal Poly. First, I want to thank my wife Amanda who has spent countless nights waiting for me to finish my work and often fell asleep in the process because I took too long. I want to apologize to my daughter Lillian for the many hours which I spent sitting at a computer instead of helping her litter toys around the apartment. I want to say thanks to my parents Mark and Darlene for supporting me in my five years at Cal Poly as well as the Navy and the Department of Naval Research for supporting me over the first summer of research on CCW. I would also like to say thanks to the rest of my family who continually asked me "so what are you doing?" and "so when will you be finished." It's finished now.

I would like to thank Dr. Marshall for the years of guidance and direction which he provided on this project. Thanks to Scott Turner for his companionship in a building without windows for an entire summer. This project would have been much different without him and the GPs. I am grateful for the other two guys who spent their days in the ATL along with Scott and me: Brian Saponas and Robert Perry. Many hours of gridding, coding, solving, and analysis was performed alongside these people and everyone was always willing to help out.

Thanks to Cal Poly for the 26 computers and the 90 plus cores on which this research were performed. Of those 26 computers, I am proud to say I crashed all but three, which were the three Windows machines which I used to write this report. And finally, I'd like to thank SSH, which provided the means for me to crash those 23 computers in five different buildings without having to leave my chair in the ATL. 


\section{Table of Contents}

List of Tables................................................................................. viii

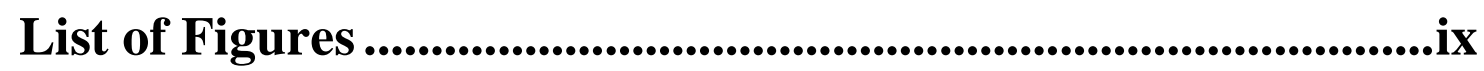

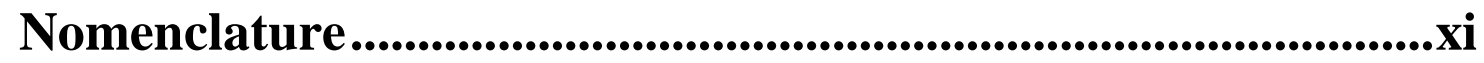

1 Introduction .......................................................................1

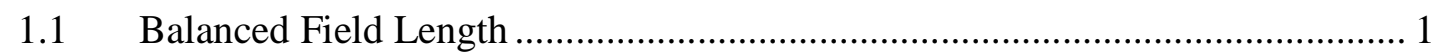

1.2 Problems with BFL Calculations for Powered Lift Aircraft.............................. 4

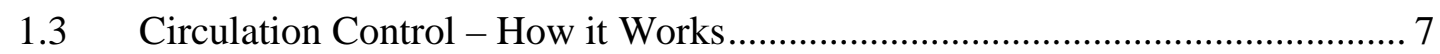

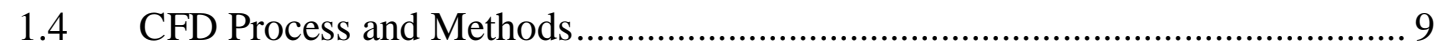

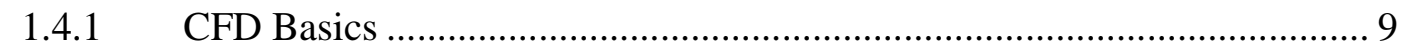

1.4.2 Computational use of Governing Equations ........................................ 11

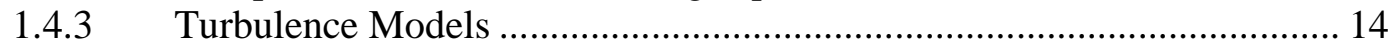

1.5 Current Research on Circulation Control .................................................. 18

1.5.1 Circulation Control and Turbulence Models .......................................... 18

2 Research of de la Montoya ....................................................29

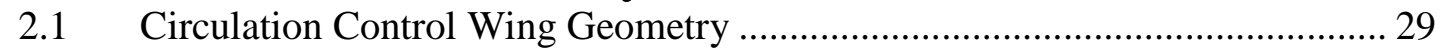

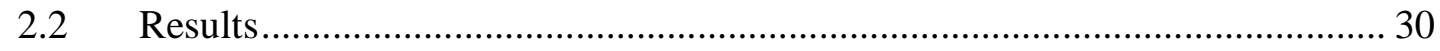

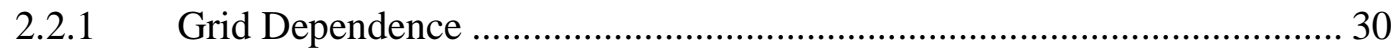

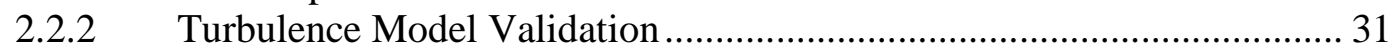

2.2.3 Three-Dimensional Analysis ............................................................ 32

3 Turbulence Model Validation ...............................................34

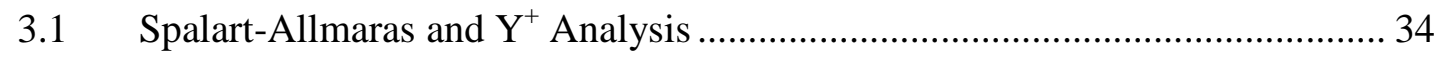

3.2 Turbulence Model Separation Verification .................................................... 35

4 Design of Experiments .............................................................41

5 Modeling and Simulation ....................................................44

5.1 Metamodeling and Gaussian Processes .......................................................... 44

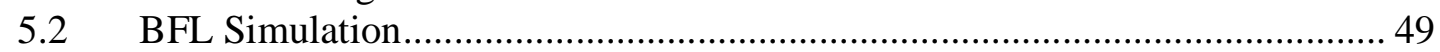

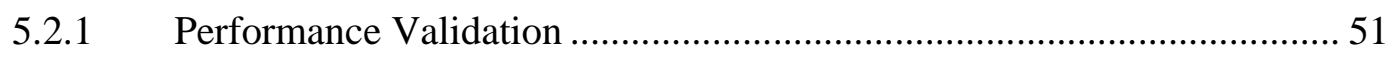

6 Preliminary Model ..................................................................55

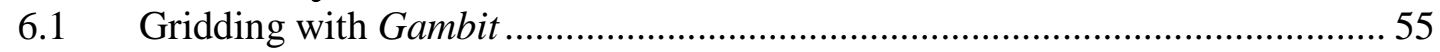

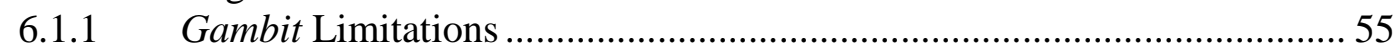

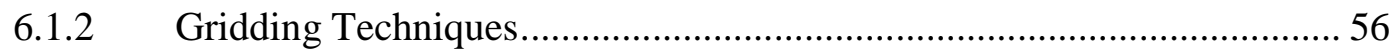

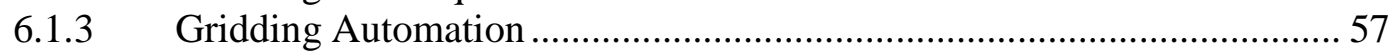

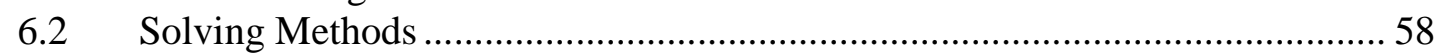

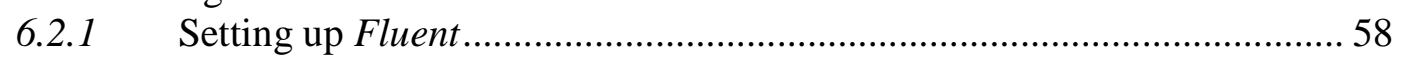

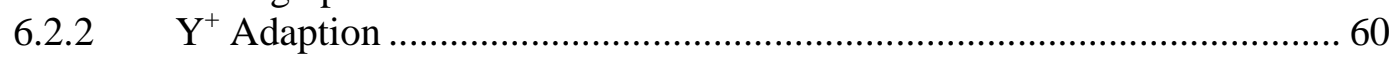

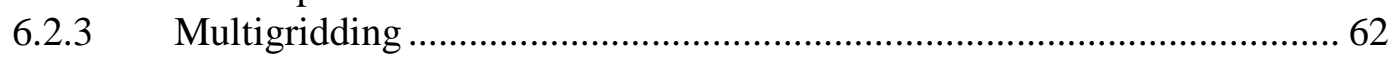

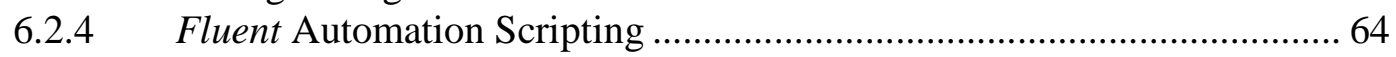

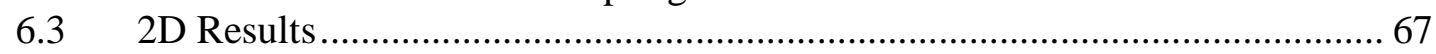

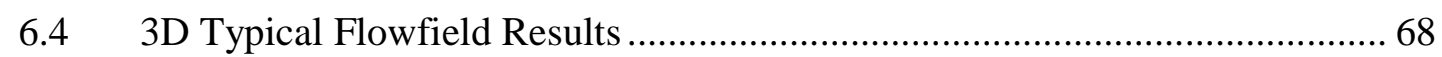

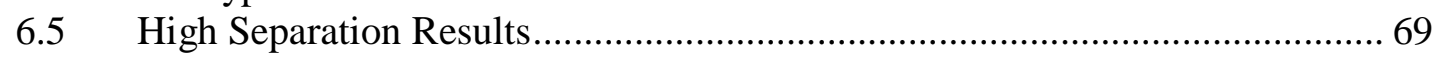

6.6 Preliminary Performance of CCW STOL Aircraft ........................................ 71 
7 Interim Model .....................................................................78

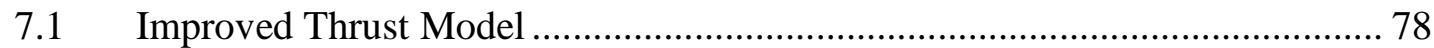

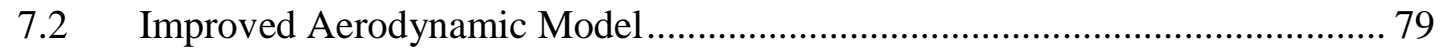

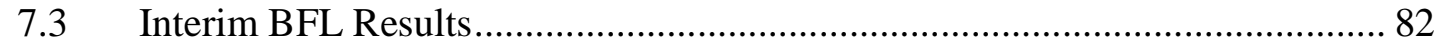

8 Final Model ...........................................................................85

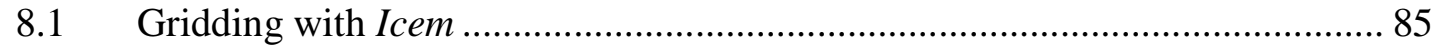

8.2 Solution Comparisons and Grid Improvements............................................ 93

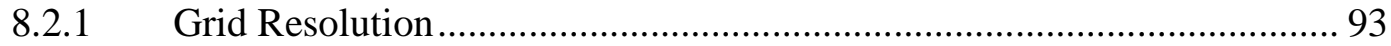

8.2.2 Flowfield Comparisons and the Boundary Layer ................................. 95

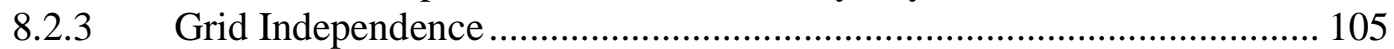

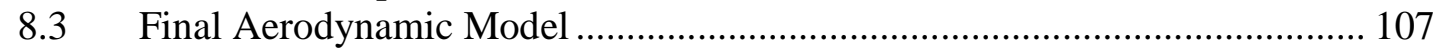

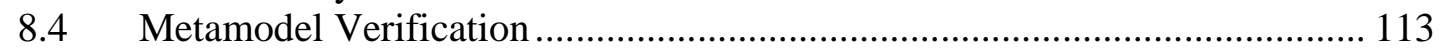

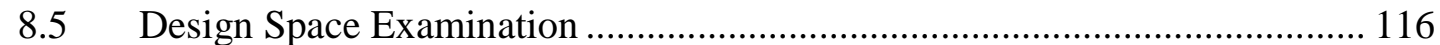

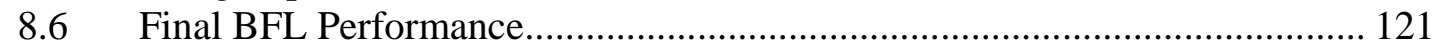

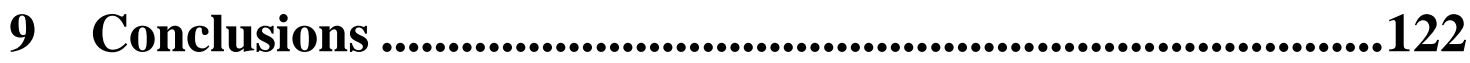

10 Future Work ...................................................................125

References .............................................................................................126

Appendix A - Mesh-Sender Script ..........................................128

Appendix B - Fluent-Setup-Run Script Documentation .......134

Appendix C - Example Template File .......................................145

Appendix D - Modified Rotation Calculations ........................151 


\section{List of Tables}

Table 3-1: Turbulence Model Results for Separated Case ............................................. 38

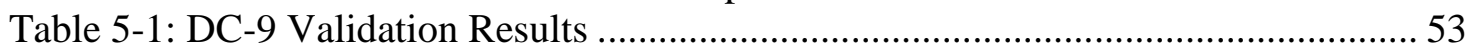

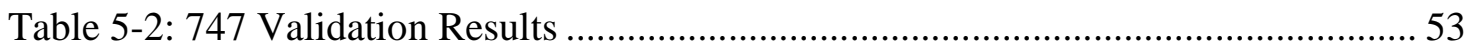

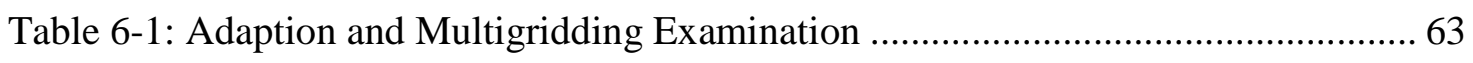

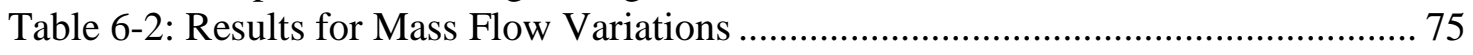

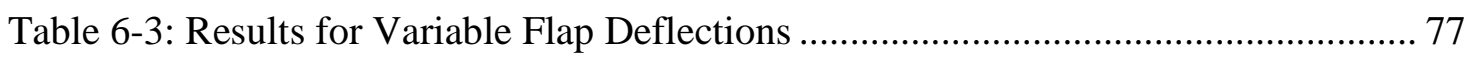

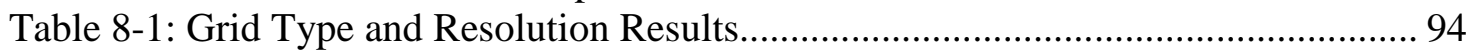

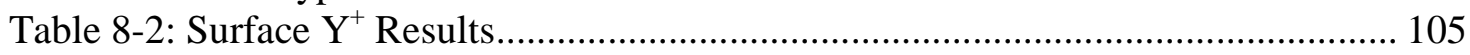

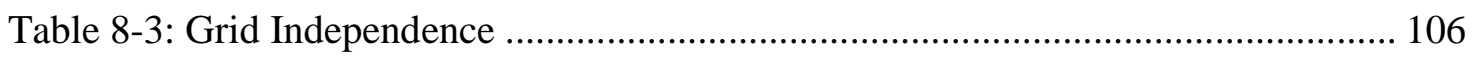




\section{List of Figures}

Figure 1-1: McDonnell Douglas C-17 (left) and Boeing Y-C 14 (right)......................... 1

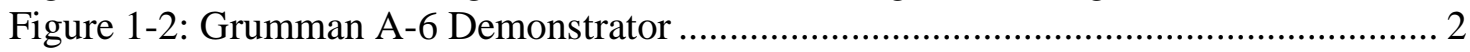

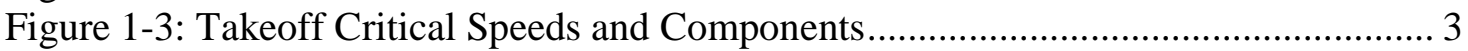

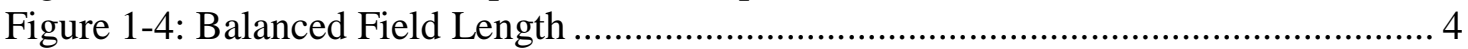

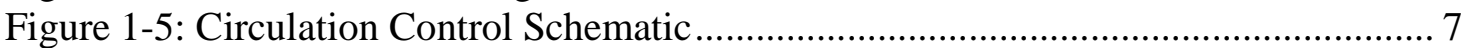

Figure 1-6: CC Trailing Edge Devices used on the A6 Demonstrator ............................... 8

Figure 1-7: Zones for a Typical Boundary Layer .............................................................. 15

Figure 1-8: CC Airfoil with Various Trailing Edges and Variable Slot Heights.............. 19

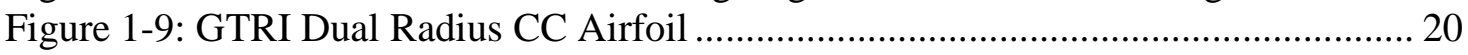

Figure 1-10: Mostly Linear Relationship between Angle of Attack and Lift.................. 21

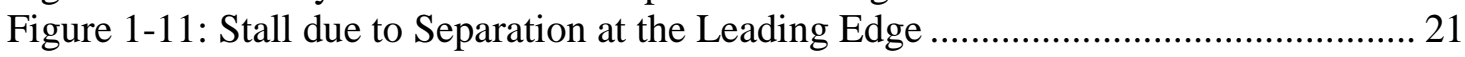

Figure 1-12: Fluent Results Compared to Experimental .............................................. 22

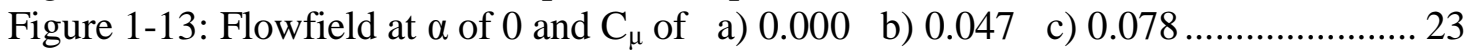

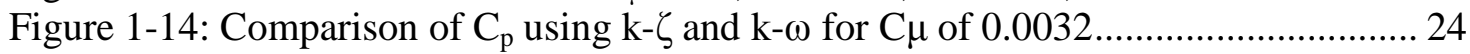

Figure 1-15: Coanda Surface Streamlines for $C \mu$ of 0.0032 of left: $k-\zeta$ and right: $k-\omega \ldots 25$

Figure 1-16: Differing Flow Results for left: Laminar and right: Turbulent jet flows ..... 25

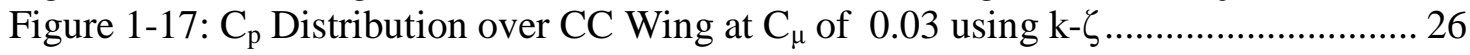

Figure 1-18: Non-Physical Separation at $C_{\mu}$ of 0.1 using left: $k-\zeta$ and right: $k-\omega$ SST $\ldots 27$

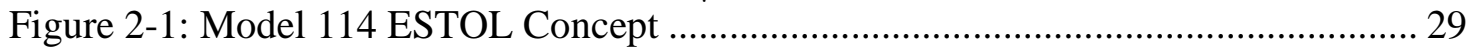

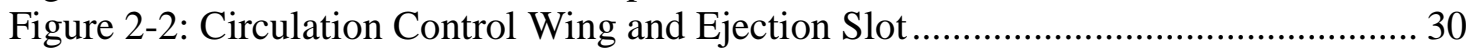

Figure 2-3: Montoya's Spalart-Allmaras Validation ................................................... 31

Figure 2-4: Lift Variation vs. Angle of Attack at Various Blowing Coefficients ............ 32

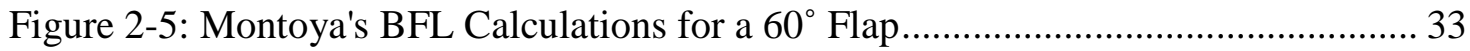

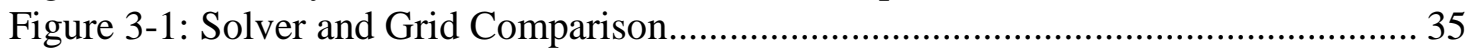

Figure 3-2: Fine Grid with $\mathrm{Y}^{+}$near 1 using Boundary Layer Cells .................................. 36

Figure 3-3: K- $\varepsilon$ Solution with Enhanced Wall Treatment (velocity streamlines m/s)...... 37

Figure 3-4: Periodic Behavior Due to Vortex Shedding with Coarse Grid ...................... 37

Figure 3-5: Vortex Shedding in Coarse SA Solution (velocity streamlines, $\mathrm{m} / \mathrm{s}$ ) ............ 38

Figure 3-6: Trailing Edge Separation Comparison (velocity streamlines, m/s) ............... 40

Figure 5-1: Complex Behavior modeled by Gaussian Process........................................ 46

Figure 5-2: Illustration of the use of Length Scale in Gaussian Processes ........................ 47

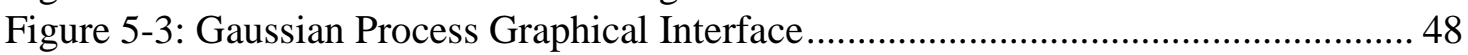

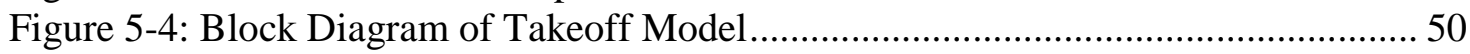

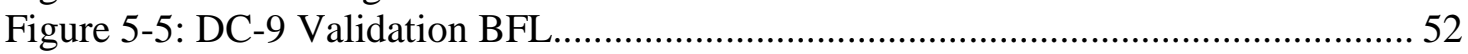

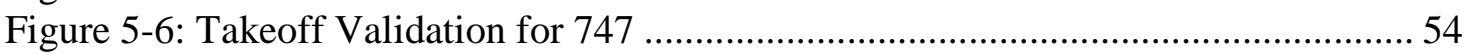

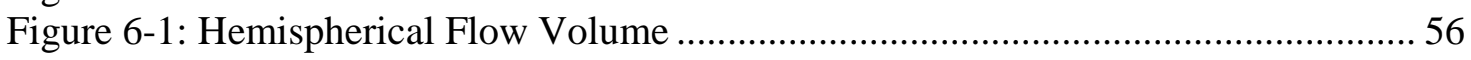

Figure 6-2: Example of a Grid Generated over the Circulation Control Wing ................. 58

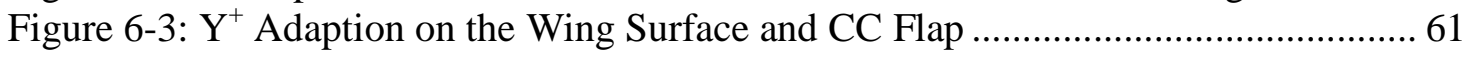

Figure 6-4: Typical CC Streamlines in 2-D, colored by Mach number...............................6 68

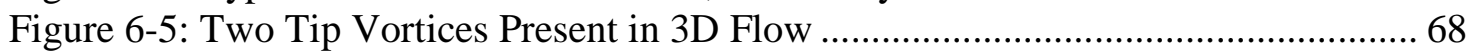

Figure 6-6: 3D Streamlines and Contours Illustrating Severe Separation ......................... 70

Figure 6-7: 2D Representation of Figure 6-6 (velocity streamlines, $\mathrm{m} / \mathrm{s}$ ) ….................... 70

Figure 6-8: STOL Transport used in CCW BFL Model ................................................ 71

Figure 6-9: BFLs for STOL Concept with varying Mass Flow and Flap Deflection ....... 72 
Figure 6-10: L/D of a Takeoff with Variations in Mass Flow Rate................................. 74

Figure 6-11: Variations in a) $C_{L}$ and b) $C_{D}$ over Takeoff with Varying Mass Flow ........ 74

Figure 6-12: L/D throughout Takeoff with Flap Variations ................................................ 76

Figure 6-13: Variations in a) $C_{L}$ and b) $C_{D}$ during Takeoff with Flap Variations............. 76

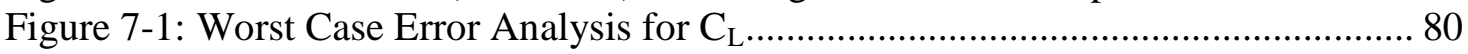

Figure 7-2: Metamodel for CL for the Preliminary Results ......................................... 81

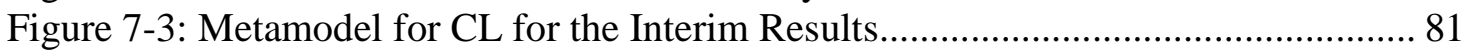

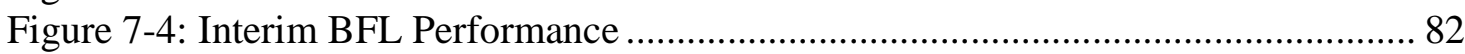

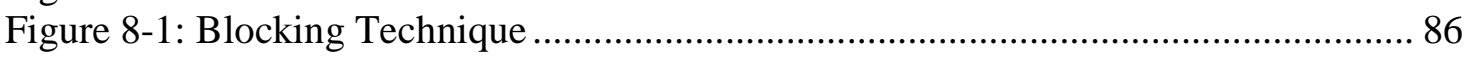

Figure 8-2: Composite Unstructured and Structured Grid............................................ 87

Figure 8-3: Boundary Layer and Tetrahedral Growth Parameters .................................. 87

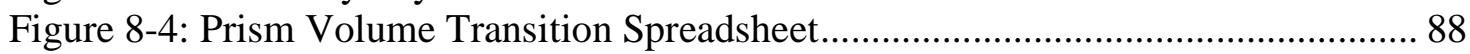

Figure 8-5: Boundary Layer Cells Turning Around Jet Slot ............................................. 89

Figure 8-6: Refinement and Boundary Layer Cells at Flap Junction .............................. 90

Figure 8-7: Cut-plane Displaying Hex-Core Conversion ............................................... 92

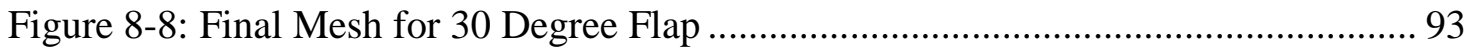

Figure 8-9: $\mathrm{C}_{\mathrm{p}}$ Contours for left: Gambit Mesh and right: Icem Mesh ........................... 96

Figure 8-10: $\mathrm{C}_{\mathrm{p}}$ Contours for top: Gambit Solutions and bottom: Icem solution .............. 97

Figure 8-11: Velocity Contours of top: Gambit and bottom: Icem Solutions (m/s) ......... 98

Figure 8-12: Velocity Slices (m/s) of the wake for top 3: Gambit, bottom 3: Icem ......... 99

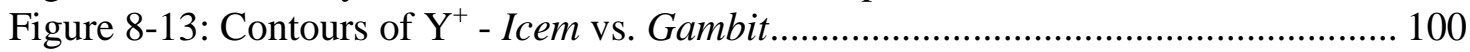

Figure 8-14: Histogram of surface $\mathrm{Y}^{+}$for refined Icem Solution .................................. 101

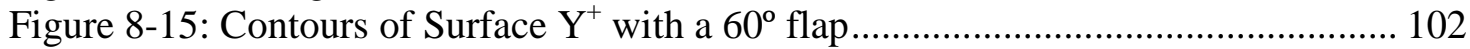

Figure 8-16: Histogram of First Attempt to Achieve Good Y+ Values .......................... 103

Figure 8-17: Histogram of Second Attempt to Achieve Good Y+ Values ...................... 104

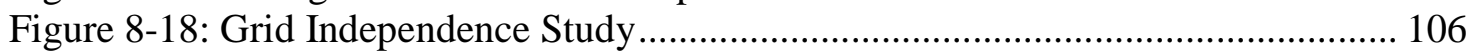

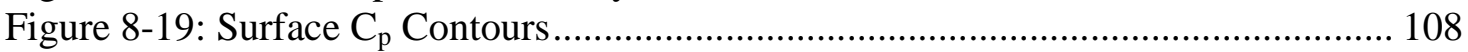

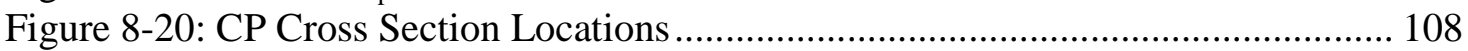

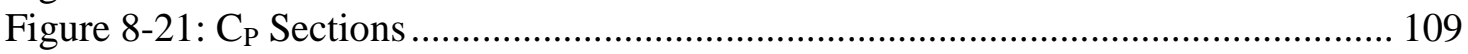

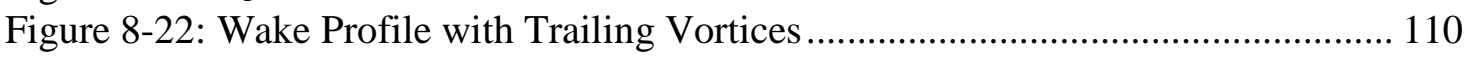

Figure 8-23: Error Analysis for Final Metamodel ......................................................... 111

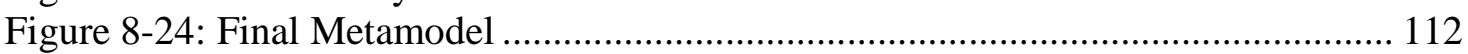

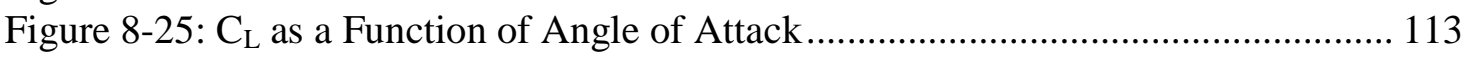

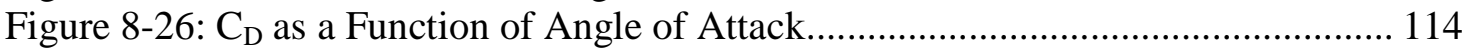

Figure 8-27: Non-Linear Relationship of $C_{D}$ and Angle of Attack ............................ 115

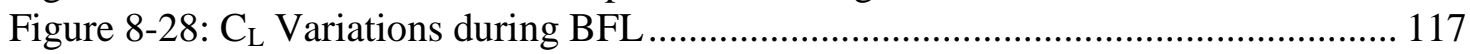

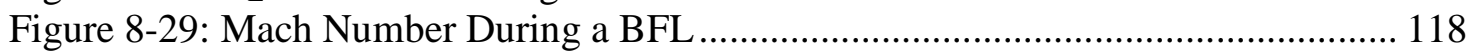

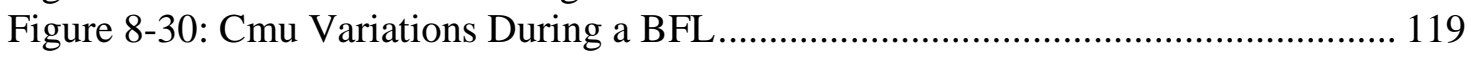

Figure 8-31: BFL (ft) vs. Mass Flow Rate (kg/s) for all Three Models with $60^{\circ}$ Flap ... 121 


\section{Nomenclature}

\begin{tabular}{|c|c|c|c|}
\hline A & $=$ area & & \\
\hline $\mathrm{a}$ & $=$ speed of sound & Subscr & \\
\hline AoA & $=$ angle-of-attack & & \\
\hline BFL & $=$ balanced field length & 1 & $=$ decision or critical speed \\
\hline BPR & $=$ bypass ratio & 2 & $=$ safety speed \\
\hline$c$ & $=$ chord length & AV & $=$ available \\
\hline$C_{D}$ & $\begin{aligned}= & \text { drag coefficient, } \\
& C_{D}=D /\left(1 / 2 \rho V^{2} S_{r e f}\right)\end{aligned}$ & $\begin{array}{l}\text { climb } \\
\text { cruise }\end{array}$ & $\begin{array}{l}=\text { climb segment } \\
=\text { cruise }\end{array}$ \\
\hline$C_{L}$ & $\begin{aligned}= & \text { lift coefficient, } \\
& C_{L}=L /\left(1 / 2 \rho V^{2} S_{r e f}\right)\end{aligned}$ & $\begin{array}{l}\mathrm{i} \\
\mathrm{i}, \mathrm{j}\end{array}$ & $\begin{array}{l}=\text { induced } \\
=\text { indexing }\end{array}$ \\
\hline$C_{P}$ & $\begin{aligned}= & \text { pressure coefficient } \\
& C_{\mathrm{P}}=\mathrm{p}-\mathrm{p}_{\infty} /\left(1 / 2 \rho \mathrm{V}^{2}\right)\end{aligned}$ & $\begin{array}{l}\text { jet } \\
\text { L }\end{array}$ & $\begin{array}{l}=\text { ejection slot } \\
=\text { landing }\end{array}$ \\
\hline$C_{\mu}$ & $\begin{aligned}= & \text { blowing coefficient } \\
& \mathrm{C}_{\mu}=\dot{m} V_{j e t} /\left(1 / 2 \rho \mathrm{V}^{2} \mathrm{~S}_{\mathrm{ref}}\right)\end{aligned}$ & $\begin{array}{l}\text { lo } \\
\max \end{array}$ & $\begin{array}{l}=\text { lift off } \\
=\text { maximum }\end{array}$ \\
\hline $\mathrm{D}$ & $=$ drag & $\mathrm{mc}$ & $=$ minimum control \\
\hline $\mathrm{F}$ & $=$ body forces & $\mathrm{mu}$ & $=$ minimum unstick \\
\hline $\mathrm{g}$ & $=$ gravity constant & $\mathrm{r}$ & $=$ rotation \\
\hline$h$ & $=$ jet slot height & ref & $=$ reference \\
\hline I & $=$ unit tensor & SL & $=$ sea level \\
\hline $\mathrm{L}$ & $=$ lift & stall & $=$ stall condition \\
\hline $\mathrm{L} / \mathrm{D}$ & $=$ lift to drag ratio & TO & $=$ take-off \\
\hline $\mathrm{M}$ & $=$ Mach number & $\infty$ & $=$ freestream \\
\hline$\dot{m}$ & $=$ mass flow rate & & \\
\hline $\mathrm{p}$ & $=$ static pressure & & \\
\hline$R$ & $=$ universal gas constant & & \\
\hline$S_{r e f}$ & $=$ wing reference area & & \\
\hline $\mathrm{T}$ & $=$ static thrust & & \\
\hline$T$ & $=$ temperature & & \\
\hline $\mathrm{t}$ & $=$ time & & \\
\hline TOGW & $=$ take-off gross weight & & \\
\hline $\mathrm{V}, \mathrm{V}$ & $=$ velocity & & \\
\hline $\mathrm{W}$ & $=$ weight & & \\
\hline $\mathrm{x}$ & $=$ longitudinal position & & \\
\hline $\mathrm{x} / \mathrm{c}$ & $=$ chord-wise position & & \\
\hline $\mathrm{y}$ & $=$ lateral position & & \\
\hline $\mathrm{Z}$ & $=$ elevation position & & \\
\hline$\mu$ & $=$ molecular viscosity & & \\
\hline$\mu_{\text {brake }}$ & $=$ braking coefficient & & \\
\hline$v$ & $=$ kinematic viscosity & & \\
\hline$\rho$ & $=$ density & & \\
\hline
\end{tabular}




\section{Introduction}

\subsection{Balanced Field Length}

Takeoff is frequently a primary constraint on an aircraft during the initial design phase, especially in the case of short takeoff and landing designs. These designs require large amounts of lift which are often unattainable to even the most sophisticated flap and slat system. McDonnell Douglas' C-17 utilizes externally blown flaps (EBF) to get its short takeoff while one of Boeing's earlier designs, the YC-14, employed upper surface blowing (USB). These aircraft are pictured in Figure 1-1.

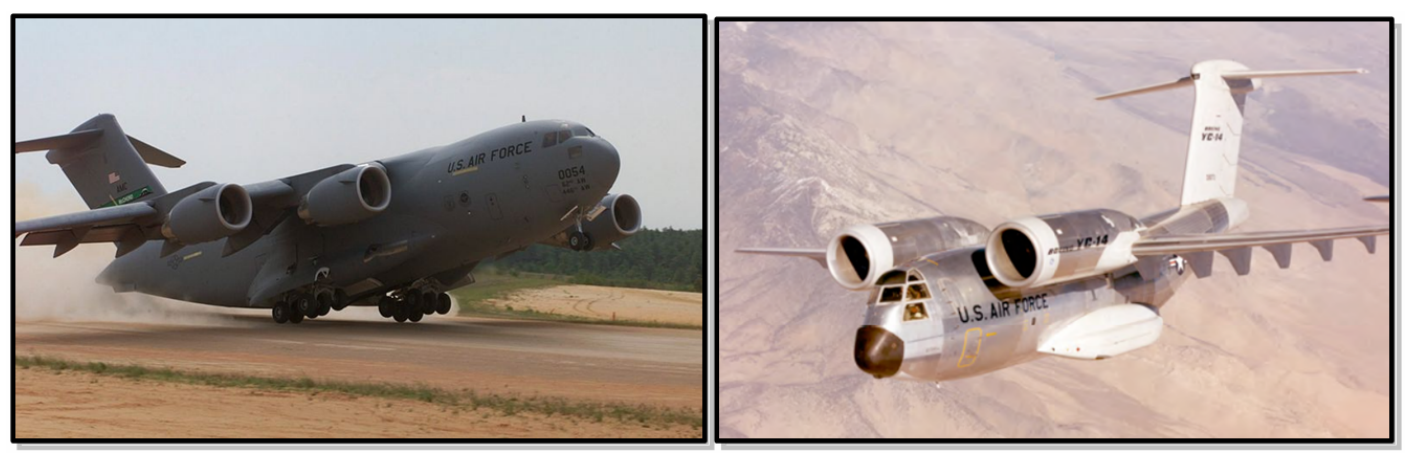

Figure 1-1: McDonnell Douglas C-17 ${ }^{1}$ (left) and Boeing Y-C $14^{2}$ (right)

Although EBF and USB can generate similar amounts of lift, other details including survivability, maintenance, and cruise performance differ greatly between the two. A third high lift technique which, like USB, utilizes a Coanda effect to generate lift is circulation control. It works by ejecting a thin sheet of air about a rounded trailing edge which in turn induces supercirculation and provides artificial camber without requiring enormous and mechanically complicated flap systems. Although no production aircraft have been built using circulation control, in 1979 the Navy's modified A-6, as seen in 
Figure $1-2^{3}$, successfully demonstrated the system and achieved approach speeds $35 \%$ slower and landing rolls $37 \%$ shorter $^{4}$ than the standard A- 6 .

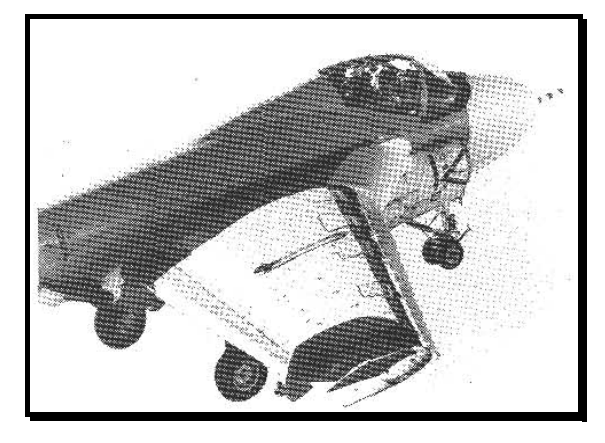

Figure 1-2: Grumman A-6 Demonstrator ${ }^{3}$

Each of the previously mentioned aircraft were specifically designed or modified to takeoff in extremely short distances and their designs revolved heavily around those requirements. In order to properly understand how to predict the takeoff performance of a short takeoff and landing (STOL) aircraft, it is necessary to clearly understand the details and components of a takeoff and balanced field length (BFL). Takeoff performance can best be analyzed by decomposing the takeoff run into smaller segments. The major segments include the ground roll, the transition phase, and the climb phase. The separation points between these segments are usually determined by critical velocities. These velocities are closely coupled with the lift of the aircraft and are either estimated as a function of the stall speed or can be calculated explicitly if enough information is known about the aircraft. A summary of the different speeds and their locations can be seen in Figure 1-3 


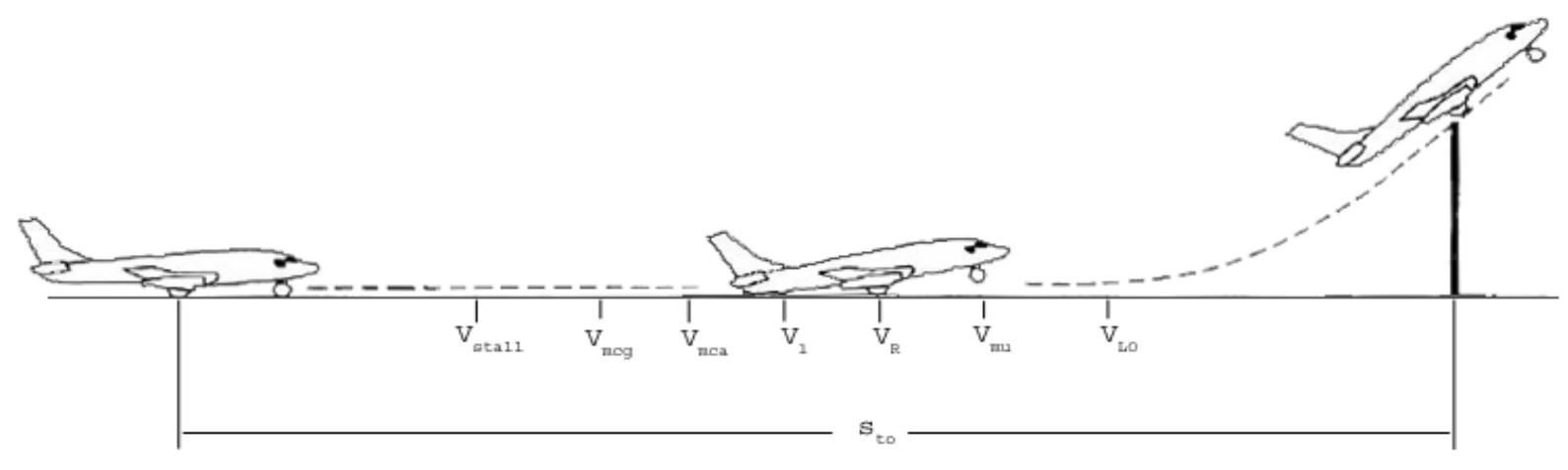

Figure 1-3: Takeoff Critical Speeds and Components ${ }^{5}$

All of the critical speeds during the takeoff run have been defined by the FAA ${ }^{6}$ in order to allow for minimum standards to be achieved by certified aircraft. The regulations therein detail information on most any circumstance including required pilot skill level, braking coefficients for aborted takeoffs, one-engine inoperative (OEI) requirements, and much more. The most critical point in the takeoff run is defined as $\mathrm{V}_{1}$ by most authors and is called the decision or critical speed. This speed denotes the maximum velocity at which if an engine failed, the pilot still has time to abort the takeoff and stop safely. This is where the term "Balanced Field Length" originates. The takeoff distance is said to be "balanced" if the braking distance is equal to the takeoff distance for an engine out scenario, as seen in Figure 1-4 from Torenbeek ${ }^{7}$. There is no set relationship as to how the decision speed is related to the stall speed. However, the decision speed is bounded on the lower end by the minimum control speed, $\mathrm{V}_{\mathrm{mc}}$, which is the minimum speed required for yaw control in the case of engine failure and on the upper end by the rotation speed, $\mathrm{V}_{\mathrm{r}}$. The speed at which rotation occurs is defined as being greater than $1.05 \mathrm{~V}_{\mathrm{mc}}$ and less than the liftoff speed". The "minimum unstick speed", or $\mathrm{V}_{\mathrm{mu}}$ can be defined as "the airspeed at and above which it can be demonstrated 
by means of flight tests that the aircraft can safely leave the ground and continue the takeoff."7

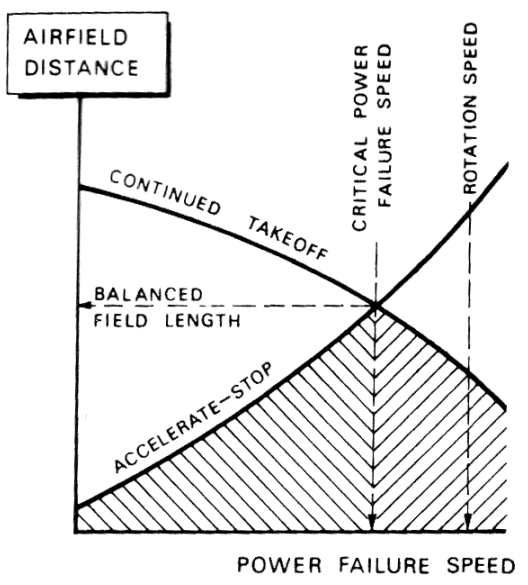

Figure 1-4: Balanced Field Length ${ }^{7}$

This velocity can also be estimated as the speed at which the fuselage tail can strike the ground prior to liftoff. Once the aircraft has reached this point, liftoff then occurs at $\mathrm{V}_{\text {lo }}$ which is usually around $1.1 \mathrm{~V}_{\text {stall. }}$. The final critical speed occurs upon clearing the 35 or 50 foot obstacle and is defined as the safety speed $V_{2}$. Most authors define the minimum $\mathrm{V}_{2}$ to be $1.2 \mathrm{~V}_{\text {stall }}$. With all of the details of a takeoff and balanced field length clearly defined, it is necessary to discuss how they are calculated in preliminary aircraft design and the limitations of those calculations with respect to any means of powered lift.

\subsection{Problems with BFL Calculations for Powered Lift Aircraft}

For preliminary design there is a serious lack in fidelity for balanced field length calculations. The traditional design texts rely on the balanced field length equation from Torenbeek's design text ${ }^{7}$, which is an equation based on the compilation of aircraft flight data. Equation(1) is useful when the aircraft falls within the range of the aircraft used to 
calibrate the equation; however, it becomes inaccurate when moving into the STOL transport design space.

$$
B F L=\frac{0.863}{1+2.3 * \Delta \gamma_{2}}\left\{\frac{W_{T O} / S}{\rho g C_{L_{2}}}+h_{t o}\right\}\left(\frac{1}{\bar{T} / W_{T O}-\mu^{\prime}}+2.7\right)+\frac{\Delta S_{t o}}{\sqrt{\sigma}}
$$

There are very few data points for which to calibrate the equation and many powered lift methods have complex lift and drag profiles across the length of the takeoff trajectory that are not captured with the simple equation. In short, a more complex method is required for balanced field length calculations on aircraft where that performance is a driving design factor.

One specific problem is the dependence of the balanced field length on the climb $C_{L}$. In the Torenbeek ${ }^{7}$ equation, the $C_{L}$ for climb is calculated from the $C_{L, m a x}$, which means essentially the equation is based on a constant lift characteristic number. This presents a significant problem when dealing with powered lift aircraft and particularly CCW aircraft. A common approach for measuring the amount of blowing from an ejection slot has been developed in the form of $\mathrm{C}_{\mu}$. The mass flow rate is coupled together with the jet velocity and nondimensionalized by the freestream dynamic pressure and wing area as shown in $\mathrm{Eq}(2)$.

$$
C_{\mu}=\frac{\dot{m} V_{j e t}}{q_{\infty} S}
$$

More details as to how the blowing coefficient affects $\mathrm{CC}$ performance will be provided in the following sections. The blowing coefficient is a function of the freestream dynamic pressure and consequently is a function of the freestream Mach 
number. At low Mach numbers, the blowing coefficient is high for a constant mass flow and at high Mach numbers the blowing coefficient is low. Since the lift coefficient is strongly based on the blowing coefficient, the lift coefficient becomes strongly coupled with the Mach number, which produces high $C_{L}$ and $C_{D}$ at low Mach numbers and lower values at takeoff speeds. This contrasts with traditional aircraft that have relatively constant lift coefficients over the ground run. Fortunately this does not completely invalidate the equation because at low Mach numbers where these $C_{L}$ and $C_{D}$ variation are greatest, the dynamic pressures are also low which result in forces of less magnitude. However, the Torenbeek ${ }^{7}$ equation is not equipped to deal with any kind of variation in $\mathrm{C}_{\mathrm{L}}$.

Another substantial problem with the equation is the choice of a $\mathrm{C}_{\mathrm{L}, \max }$ for a powered lift aircraft. As mentioned above, the dependency of the lift coefficient on more than angle of attack complicates the actual lift and drag forces on the aircraft. Since the Torenbeek ${ }^{7}$ equation is a function of only a single $\mathrm{C}_{\mathrm{L}}$ its lift model is at the mercy of that number, which for a powered-lift aircraft is not a simple calculation. For a traditional aircraft the maximum lift coefficient is based solely on angle of attack, which provides a buffer when rotating an aircraft for takeoff. For a powered lift aircraft that reference point needs to be a function of the freestream Mach number and the amount of blowing, both of which strongly affect the actual maximum $\mathrm{C}_{\mathrm{L}}$. The rotation velocity seems like a logical place to define the $\mathrm{C}_{\mathrm{L}, \max }$ for powered lift aircraft, but in traditional preliminary design the rotation velocity is defined by a percentage of the stall velocity, which is calculated from $\mathrm{C}_{\mathrm{Lmax}}$. Thus, this circular logic makes it difficult to calculate a $\mathrm{C}_{\mathrm{Lmax}}$ for a 
powered lift aircraft in preliminary design. A solution to this particular problem is discussed in a later section.

\subsection{Circulation Control - How it Works}

The idea of using pneumatic devices to augment airfoils has been around since the $1930 s^{8}$. Most of these early designs consisted of either jet flaps or blown flaps which utilized a sheet of air ejected on a flap or at a given angle. The term "circulation control" came by extending the performance of those previous designs by ejecting the flow over a rounded trailing edge as can be seen in Figure 1-5 from reference 8.

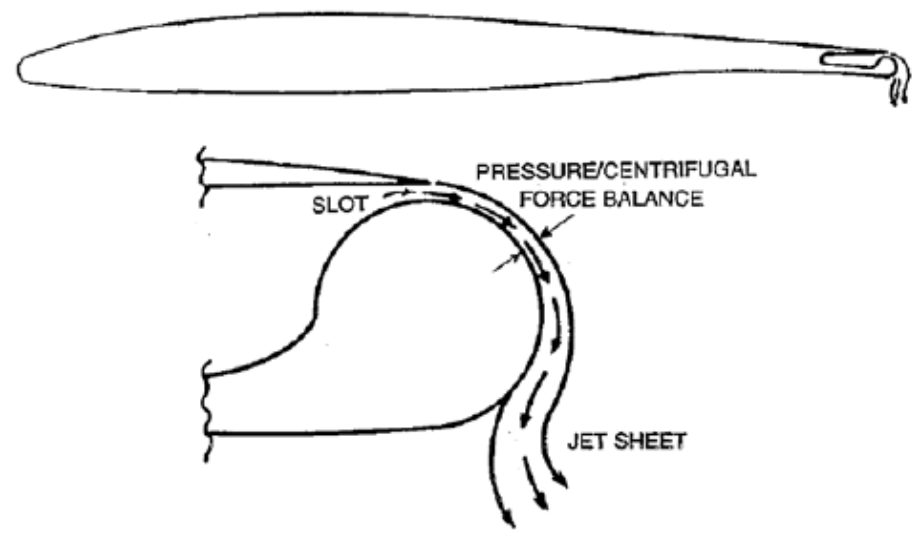

Figure 1-5: Circulation Control Schematic ${ }^{8}$

The Coanda effect holds the sheet of ejected air to the rounded surfaces and that sheet in turn entrains the external flow around it and directs it downward. The downward deflection can be thought of as a pneumatic flap which effectively increases the camber and lift of the airfoil or wing. Some of the early benefits of CC included the ability to achieve high lift with little flaps or even a fixed trailing edge and the ability to increase lift without a change in angle of attack. Both of these characteristics prove to be desirable in the design of a STOL transport for obvious reasons. 
A great deal of effort has gone into the design of the trailing edge of CC airfoils. Although it is well established that a rounded trailing edge performs well, it is not well known how large of a radius or even if it should be circular. In addition to the difficulty of deciding on the shape of the trailing edge, another problem is determining how to best create that shape. Some of the common methods of how this has been achieved are illustrated in Figure 1-69.

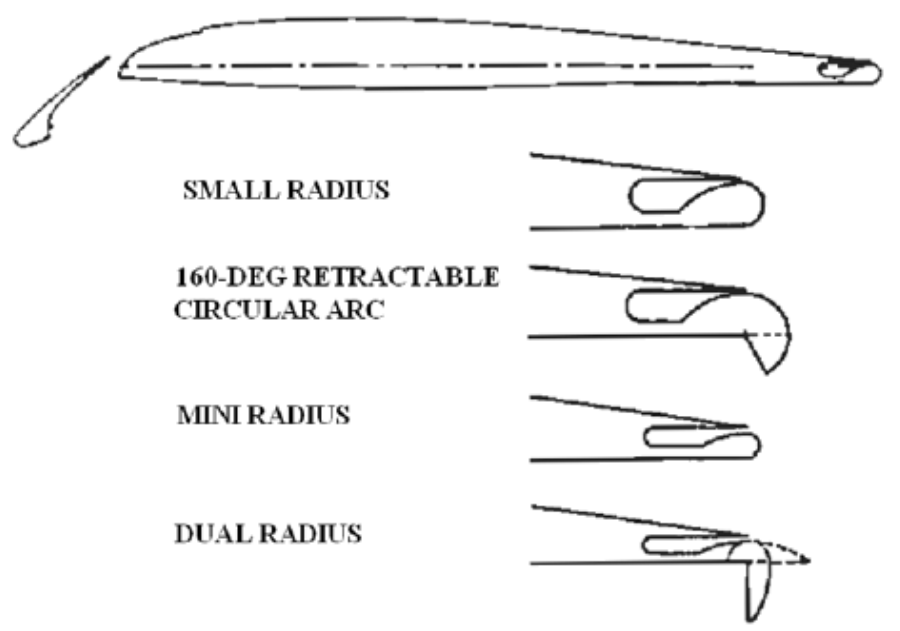

Figure 1-6: CC Trailing Edge Devices used on the A6 Demonstrator?

A fixed rounded trailing edge adds a significant amount of drag during cruise while the mechanical systems required to create the Coanda surface can be complicated and can therefore eliminate the benefit of the mechanically simpler pneumatic flap. The Navy's DTNSRDC and Grumman took two approaches ${ }^{9}$ to the drag problem in the 1980s. One method was to have a fixed rounded trailing edge of $0.009 \mathrm{c}$ which was found to have good lifting capabilities and better drag results than some of the larger trailing edges. The other solution was to have a simple CCW flap with a curved upper surface and sharp trailing edge. When the flap is deflected, it exposes the smaller to the two radii of which the flow would be tangentially ejected. After passing over the first radius, the 
flow then travels past the lager radius created by the flap upper surface. This dual radius combination provides a large Coanda surface to facilitate the $\mathrm{CC}$ and allows for a sharp trailing edge for cruise flight. For these reasons, the dual radius trailing edge was used for this study.

\subsection{CFD Process and Methods}

Before the current CFD research is presented, it is important to first describe what the overall CFD process aims to accomplish and how it is performed. This section will briefly overview these principles as well as how the governing equations along with turbulence models are used to numerically solve fluid flow. The process in performing CFD is in actuality very similar to performing a physical experiment. A problem must be adapted from the real world application to a simplified version which can be tested, a prototype model must be fabricated, and the fluid flow over the model must be accurately simulated.

\subsubsection{CFD Basics}

The physical model for CFD cases most often is generated via CAD and then imported into meshing software. The quality of the CAD model is of key importance to the results of the CFD solution. The major physical features under consideration must be present in order to produce the required flow effects. However, it is essential that care is taken to eliminate cracks, holes, and excessively complicated geometry which are of little consequence to the desired flow features. If this step is neglected, the quality of the mesh and therefore the final CFD results will be greatly reduced or even not reach steady state. The analogy to experimental aerodynamics is the common practice of using clay to smooth sharp corners and other features in order to clean up the flow. 
Many simplifications must be performed in CFD as well as experimental work in order to translate a real world problem into a test situation. In addition to the geometry simplifications just mentioned, boundary conditions are another tool which can be used to simplify the solving process in CFD. For example, most airplane wings are symmetrical which means that fluid flow over one half is identical to the flow over the other. Within a CFD model, a symmetry boundary condition can be specified which in turn reduces the amount of computation required by half. This same technique is performed in wind tunnel tests which use only half of a wing. Another simplification which is relevant to circulation control involves how the ejection slot is modeled. In the real world, a complicated ducting system would be required to channel the flow from a source engine to the ejection slot. To model the aircraft engine and ducting system would require an immensely complicated model and many hours of computational time. An accurate simplification for the real world model is simply to define the ejection slot face as a mass flow inlet which can be adjusted to eject specified amounts of fluid flow into the flow field at a desired temperature and mass flow rate in $\mathrm{kg} / \mathrm{s}$. Simplifications like these, as in experimental results, if applied correctly can greatly reduce the amount of work required to obtain a solution.

Out of all of the many complicated steps required to obtain a CFD solution, the most important by far is obtaining a quality mesh. Poor mesh quality can lead to results which are skewed due to errors introduced by the grid (i.e. grid dependent), or cause a normally stable solution to diverge. Great care must be taken during the gridding process in order to limit the amount of cell skewness and large volume transitions. Where ever possible, hexahedral cells should be used in place of tetrahedrals in order to provide cells 
that have the majority of cell faces normal to the freestream direction. This reduces the amount of round off error which is introduced into the system from the fluid flux calculations through the faces of each cell. Additional meshing difficulties include providing enough resolution in areas of interest in order to capture important flow features like turbulence and shear layers. In the case of circulation control, a significant shear layer is present between the high speed flow ejected over the trailing edge which needs to be resolved as well as the standard wall effects present on the other wing surfaces. Areas like these require high refinement and much care must be taken to ensure that the important flow features are captured using an efficient number of cells. A grid independence study should be performed in order to determine the proper number of cells required to obtain accurate results.

\subsubsection{Computational use of Governing Equations}

Although CFD is a complicated process involving many hours and often many computers, it is based off of the same physical principles which are applied in ordinary physics and chemistry. These basic principles are all contained in what are called the governing equations which for simple fluid flow without chemical reactions or electromagnetic effects include the conservation of mass, momentum, and energy. Conservation of mass in three dimensions is listed as $\mathrm{Eq}(3)$.

$$
\frac{\partial \rho}{\partial t}+\frac{\partial(\rho u)}{\partial x}+\frac{\partial(\rho v)}{\partial y}+\frac{\partial(\rho w)}{\partial w}=0
$$

Three more equations come from the conservation of momentum which is also known as the Navier-Stokes equation which is expressed in its condensed form in Eq(4) . 


$$
\frac{D V}{D t}=-\frac{1}{\rho} \nabla p+g+\frac{1}{\rho} \nabla \cdot \tau_{i j}
$$

The Navier-Stokes equations apply in three dimensions and in Cartesian coordinates with compressibility effects included are given as $\mathrm{Eq}(5)$ through $\mathrm{Eq}(7)$.

$$
\begin{aligned}
& \frac{\partial(\rho u)}{\partial t}+\frac{\partial\left(\rho u^{2}\right)}{\partial x}+\frac{\partial(\rho u v)}{\partial y}+\frac{\partial(\rho u w)}{\partial z}=\frac{-\partial p}{\partial x}+\frac{\partial \tau_{x x}}{\partial x}+\frac{\partial \tau_{x y}}{\partial y}+\frac{\partial \tau_{x z}}{\partial z}+S_{x} \\
& \frac{\partial(\rho v)}{\partial t}+\frac{\partial(\rho u v)}{\partial x}+\frac{\partial\left(\rho v^{2}\right)}{\partial y}+\frac{\partial(\rho v w)}{\partial z}=\frac{-\partial p}{\partial y}+\frac{\partial \tau_{y x}}{\partial x}+\frac{\partial \tau_{y y}}{\partial y}+\frac{\partial \tau_{y z}}{\partial z}+S_{y} \\
& \frac{\partial(\rho w)}{\partial t}+\frac{\partial(\rho u w)}{\partial x}+\frac{\partial(\rho v w)}{\partial y}+\frac{\partial\left(\rho w^{2}\right)}{\partial z}=\frac{-\partial p}{\partial z}+\frac{\partial \tau_{z x}}{\partial x}+\frac{\partial \tau_{z y}}{\partial y}+\frac{\partial \tau_{z z}}{\partial z}+S_{z}
\end{aligned}
$$

Finally, the conservation of energy is listed as $\mathrm{Eq}(8)$.

$$
\begin{aligned}
& \frac{\partial(\rho E)}{\partial t}+\frac{\partial(\rho u E)}{\partial x}+\frac{\partial(\rho v E)}{\partial y}+\frac{\partial(\rho w E)}{\partial z}=-\left[\frac{\partial(u p)}{\partial x}+\frac{\partial(v p)}{\partial y}+\frac{\partial(w p)}{\partial z}\right] \\
& +\left[\frac{\partial\left(u \tau_{x x}\right)}{\partial x}+\frac{\partial\left(u \tau_{x y}\right)}{\partial y}+\frac{\partial\left(u \tau_{x z}\right)}{\partial z}\right]+\left[\frac{\partial\left(v \tau_{y x}\right)}{\partial x}+\frac{\partial\left(v \tau_{y y}\right)}{\partial y}+\frac{\partial\left(v \tau_{y z}\right)}{\partial z}\right] \\
& +\left[\frac{\partial\left(w \tau_{z x}\right)}{\partial x}+\frac{\partial\left(w \tau_{z y}\right)}{\partial y}+\frac{\partial\left(w \tau_{z z}\right)}{\partial z}\right]+k\left[\frac{\partial^{2} T}{\partial x^{2}}+\frac{\partial^{2} T}{\partial y^{2}}+\frac{\partial^{2} T}{\partial z^{2}}\right]+e+\frac{1}{2}\left(u^{2}+v^{2}+w^{2}\right)
\end{aligned}
$$

Within the five governing equations there are eight unknowns, which are:

$$
\rho, u, v, w, p, T, e, \mu
$$

Since there are more unknowns than equations, additional equations are required to obtain a solution. For external aerodynamics, the equations that are often used are the equations of state for both thermodynamics and gas, and a description of the molecular viscosity. The perfect gas relationship is shown in $\mathrm{Eq}(9)$. 


$$
P=\rho R T
$$

A relationship between the thermodynamic variables adds a seventh equation. For a perfect gas with a constant specific heat that relationship is given as $\mathrm{Eq}(10)$.

$$
e=C_{v} T
$$

The final equation required to define the system is a representation of the molecular viscosity, which for incompressible flows with small temperature gradients is given as $\operatorname{Eq}(11)$.

$$
\mu=\text { constant }
$$

With the use of these eight equations in their discritized form, the fluid flow is able to be solved in each computational cell. Because there are no analytical relationships for the fluid properties throughout a flowfield of any complexity, these computations must be performed numerically. The initial flow in each cell is normally initialized with the freestream properties. This condition is erroneous in many places in the flowfield and requires several hundreds and many times thousands of iterations for the effects of the different boundary conditions to propagate throughout the entire flowfield. The period of time while the flow properties are still changing is called the transient phase and when the properties throughout the flowfield are constant, the flow is said to have converged or reached steady state. Since wall effects contribute the vast majority of the turbulence throughout the flow, it is important to overview the different methods for resolving this turbulence. 


\subsubsection{Turbulence Models}

Although the Navier-Stokes equations are fundamental and rigorous, they are also non-linear, non-unique, complex, and difficult to solve ${ }^{10}$. Several methods have been used in an attempt to model the Navier-Stokes equations, including Direct Numerical Simulation (DNS) which solves them without approximating turbulence in either time or space. However, DNS methods require enormous amounts of computation and are impractical for most flows. As a result, several turbulence models have been developed which attempt to model the complex turbulent flow.

The turbulence found in a flowfield often originates in the boundary layer which contains the interactions between a wall and the freestream flow. In essence, the boundary layer smoothes the discontinuity between the no-slip wall surface and the fast moving freestream flow. It is very important to ensure that the boundary layer is being resolved so that the turbulent flow can be adequately predicted. One of the dimensionless parameters used to measure a boundary layer height is $\mathrm{y}^{+}$which is defined in $\mathrm{Eq}(12)$.

$$
y^{+}=\frac{y\left(\tau_{w} / \rho_{w}\right)^{1 / 2}}{v_{w}}
$$

Four regions have been defined within the boundary layer in an attempt to model its behavior: the linear viscous sublayer, the buffer layer, the fully turbulent log-law region, and the outer region ${ }^{11}$ as can be seen in Figure 1-7. For many turbulent boundary layers, the inner region has been shown to have similar characteristics which are captured by the logarithmic "law-of-the-wall." 


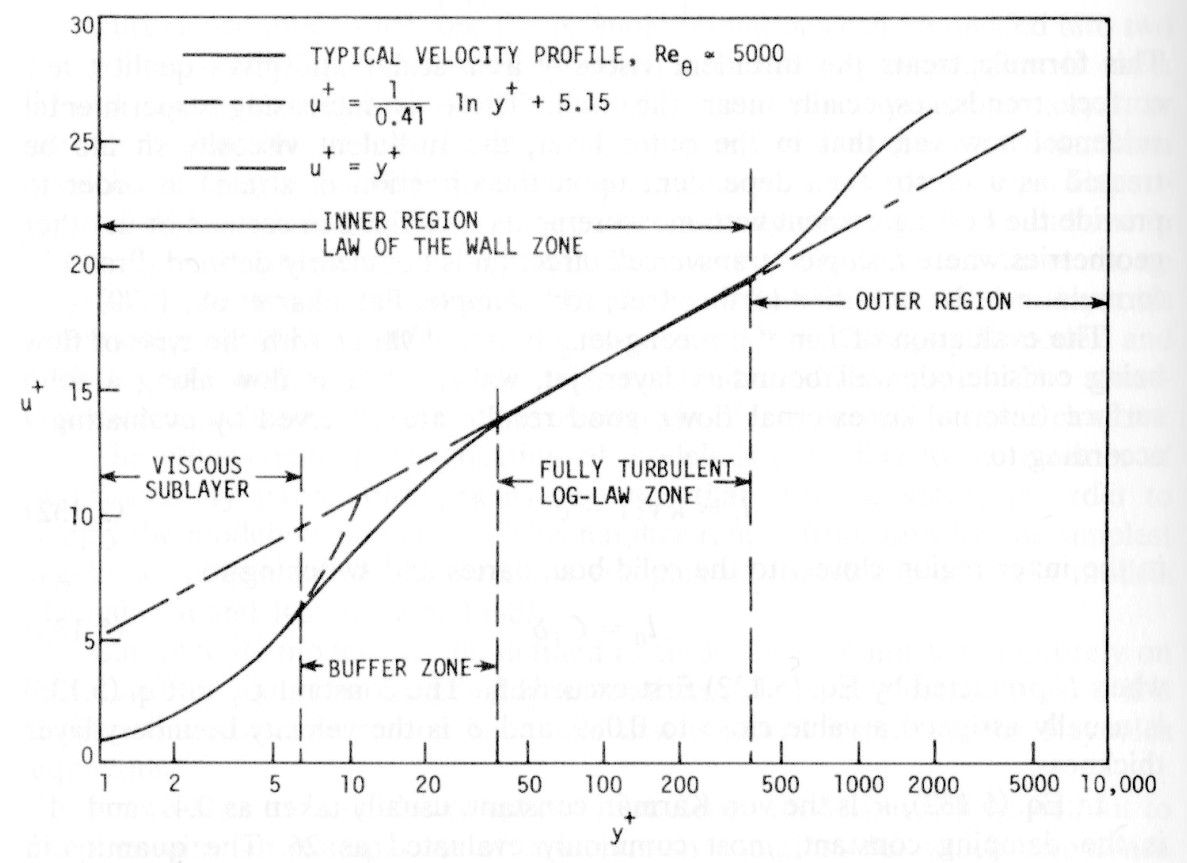

Figure 1-7: Zones for a Typical Boundary Layer ${ }^{11}$

The wall function approach to resolving the boundary layer is to use a turbulence model to predict the outer region and have the inner region inserted with its use of a form of the law-of-the-wall ${ }^{11}$. With this approach, a relatively coarse grid can be used to approximately capture the boundary layer as long as the first cell off of the wall surface lies between $30<y^{+}<200$. For a majority of this study, the wall function approach was used along with the Spalart-Allmaras one-equation turbulence model.

A turbulence model can be defined as a semi-empirical equation relating the fluctuating correlation to the mean flow variables with various constants provided from experimental investigations ${ }^{12}$. The way that the equation is expressed defines the type of turbulence model. For example, if an algebraic equation is used, it is known as a zeroequation model, while if partial differential equations (PDEs) are used, it is known as a one or two-equation model depending on how many PDEs are used. Spalart-Allmaras is 
a one-equation turbulence model which solves a transport equation, which is based off the Navier-Stokes equations, for a working variable $\bar{v}$ which is related to the eddy viscocity $^{12}$. The governing equation for this model is derived by using empiricism, dimensional analysis, Galilean invariance, and selected dependence on the molecular viscosity. One form of the Spalart-Allmaras model as taken from reference 12 is given as $\operatorname{Eq}(13)$.

$$
\begin{aligned}
& \frac{d v}{d t}=\left(\frac{1+c_{b 2}}{\sigma}\right)[\nabla(v+\bar{v}) \cdot \nabla \bar{v}]+\frac{1}{\sigma}(v+\bar{v}) \nabla^{2} \bar{v} \\
& +c_{b 1}\left(1-f_{t 2}\right) \bar{v} \bar{S}-\left[c_{w 1} f_{w}-\frac{c_{b 1}}{\kappa^{2}} f_{t 2}\right]\left[\frac{\bar{v}}{d}\right]^{2}+f_{t 1}(\Delta q)^{2}
\end{aligned}
$$

The reader should consult reference 12 for details on the specific values used for the empirical constants and functions or for further derivation of the turbulence model into computational space. Since the transport equation is a PDE, it requires initial and boundary conditions. The initial condition for $\bar{v}$ is specified to be zero up to $v_{\infty} / 10$. The boundary conditions ${ }^{12}$ are as follows:

1. At the inflow, $\bar{v}=\bar{v}_{\infty}$

2. At a solid surface, $\bar{v}=0$ and

3. At the outflow, extrapolation is used.

As stated, the majority of this study employed Spalart-Allmaras as the turbulence model along with the wall function approach. However, it was stated by Hoffmann ${ }^{12}$ that:

"Complex flowfields which include massively separated flows...the lower order turbulence models, that is the zero, half-, or one-equation models, become very complicated and often ambiguous. 
Two-equation models are developed to better represent the physics of turbulence in these types of flowfields."

Section 3.2 will deal with this type of highly separated flow and will also provide a comparison between Spalart-Allmaras and two different two-equation models: k- $\varepsilon$ and k$\omega$ SST. These two-equation models employ two transport equations which attempt to model the kinetic energy and dissipation of turbulence. The k- $\varepsilon$ model is expressed in $\mathrm{Eq}(14)$ and $\mathrm{Eq}(15)$.

$$
\begin{gathered}
\rho \frac{d k}{d t}=\frac{\partial}{\partial x_{j}}\left[\left(\mu+\frac{\mu_{t}}{\sigma_{k}}\right) \frac{\partial k}{\partial x_{j}}\right]+P_{k}-\rho \varepsilon \\
\rho \frac{d \varepsilon}{d t}=\frac{\partial}{\partial x_{j}}\left[\left(\mu+\frac{\mu_{t}}{\sigma_{\varepsilon}}\right) \frac{\partial \varepsilon}{\partial x_{j}}\right]+c_{\varepsilon 1} P_{k} \frac{\varepsilon}{k}-c_{\varepsilon 2} \rho \frac{\varepsilon^{2}}{k}
\end{gathered}
$$

The $\mathrm{k}-\omega$ SST model is a combination of the $\mathrm{k}-\varepsilon$ and baseline $\mathrm{k}-\omega$ models. This model incorporates the superior performance of the $\mathrm{k}-\omega$ model within the laminar sublayer and logarithmic regions of the boundary layer with the superior performance of k- $\varepsilon$ in the outer portion and wake region of the boundary layer. Therefore, the k- $\omega$ SST uses $\mathrm{Eq}(14)$ and $\mathrm{Eq}(15)$ in the outer wake region and $\mathrm{Eq}(16)$ and $\mathrm{Eq}(17)$ in the sublayer and $\log$ layer of the boundary layer.

$$
\begin{gathered}
\frac{\partial}{\partial t}(\bar{\rho} k)+\frac{\partial}{\partial x_{j}}\left(\bar{\rho}_{j} k\right)=\frac{\partial}{\partial x_{j}}\left[\left(\mu+\sigma_{k 1} \mu_{t}\right) \frac{\partial k}{\partial x_{j}}\right]+P_{k}-\beta^{*} \bar{\rho} k \omega \\
\frac{\partial}{\partial t}(\bar{\rho} \omega)+\frac{\partial}{\partial x_{j}}\left(\bar{\rho}_{j} \omega\right)=\frac{\partial}{\partial x_{j}}\left[\left(\mu+\sigma_{\omega 1} \mu_{t}\right) \frac{\partial \omega}{\partial x_{j}}\right]+\alpha_{1} \frac{\omega}{k} P_{k}-\beta^{*} \bar{\rho} \omega^{2}
\end{gathered}
$$


Again, for more information about the formulation of these turbulence models, see reference 12. The next section presents some of the current research with $\mathrm{CC}$ including the results with various turbulence models.

\subsection{Current Research on Circulation Control}

This thesis work began because of the author's interest in CFD and as a continuation of previous research performed by Juliana de la Montoya ${ }^{13}$. Montoya's work consisted of a detailed literature search about the history of circulation control, as well as past and present methods of performing numerical analysis. In addition, Montoya performed her own analysis as applied to a STOL transport. Some of the major highlights of Montoya's background research ${ }^{13}$ which apply to this study are included here as a reference along with additional resources relating to turbulence model performance of circulation control.

\subsubsection{Circulation Control and Turbulence Models}

In order for a CC airfoil to perform efficiently, it is important that the ejection slot height be a proper height. A study ${ }^{14}$ was performed which compared wind tunnel results and a CC airfoil with interchangeable trailing edge geometries and variable slot heights. An example of this airfoil can be seen in Figure $1-8^{14}$. Some of the major conclusions consisted of greater lift due to higher mass flow through the slot and at higher angles of attack. Also, it was found that lift decreased as Mach number increased. One other feature that was discovered was that jet slot height-to-chord ratios (h/c) above 0.0020 were much less efficient than their smaller counterparts. This piece of information was used later in the design of the CCW geometry which will be discussed in a later section. 


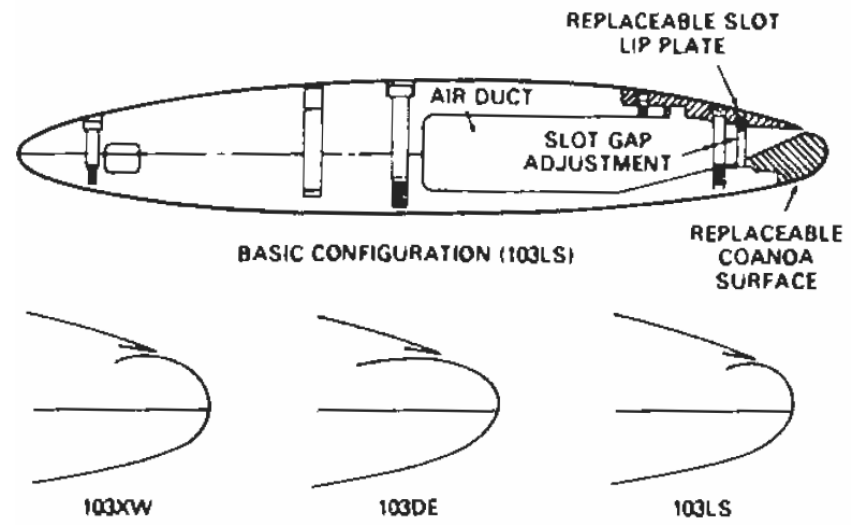

Figure 1-8: CC Airfoil with Various Trailing Edges and Variable Slot Heights ${ }^{14}$

One of the drawbacks found about a circulation control wing was that generating large amounts of lift has the tendency to have flow separate at only moderate angles of attack $^{9}$. Also, the blunt trailing edge left by the Coanda surface was found to cause additional drag during cruise conditions. To solve these drag problems, supercritical airfoils have been used because of their large leading edge radius and their reduced drag at transonic speeds. Also, because CC produces such large amounts of lift at little to no angles of attack, the separation issues can be solved by limiting the angle of attack. The drag problem associated with the blunt trailing edge was reduced significantly on the A6 Demonstrator by using a dual radius CC flap instead of a rounded trailing edge as was pictured in Figure 1-6.

A study ${ }^{15}$ of a particular supercritical airfoil created by GTRI was performed in order to evaluate the performance of the dual radius $\mathrm{CC}$ flap. This airfoil, which is pictured in Figure 1-9, was found to perform better than a fixed rounded edge airfoil due to the dual radius flap's ability to rotate. The GTRI airfoil was able to deflect more air downward and also reduce the amount of drag at cruise by rotating the flap to the desired 
position. Because of these advantages, an airfoil geometry based off of the GTRI airfoil was used in this study.

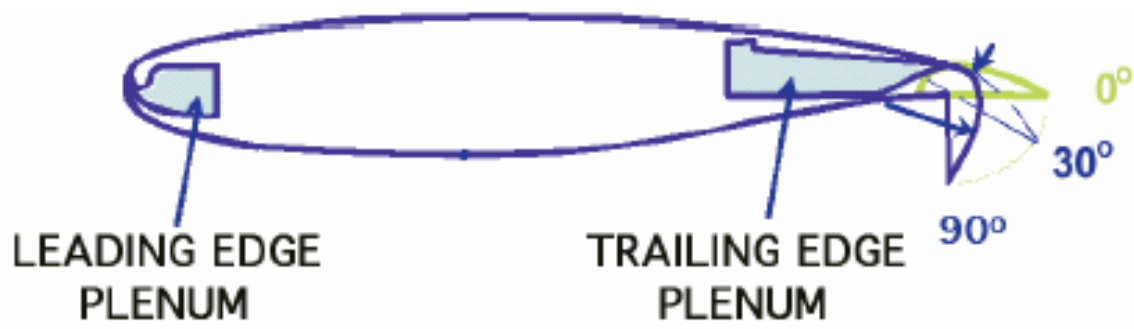

Figure 1-9: GTRI Dual Radius CC Airfoil ${ }^{15}$

A considerable amount of research has focused on which CFD solvers and turbulence models can best predict the flow phenomenon produced by circulation control. One of these studies ${ }^{16}$ was performed on the GTRI CC airfoil using and unsteady viscous flow RANS solver and the Baldwin-Lomax and Spalart-Allmaras (SA) turbulence models. These models were found to work well and produced results that showed a linear increase in lift with angle of attack, as seen in Figure 1-10 from reference 16, and also that $\mathrm{CC}$ airfoils tend to stall because of leading edge separation, as seen in Figure 1-11 from the same reference. Because of this separation effect, the GTRI study ${ }^{16}$ as well as others employed the use of leading edge blowing. In addition, results published in this paper giving the relationship between $C_{\mu}$ and $C_{1}$ were later used in this study for turbulence model validation for the GTRI airfoil which will be detailed in section 3 . 


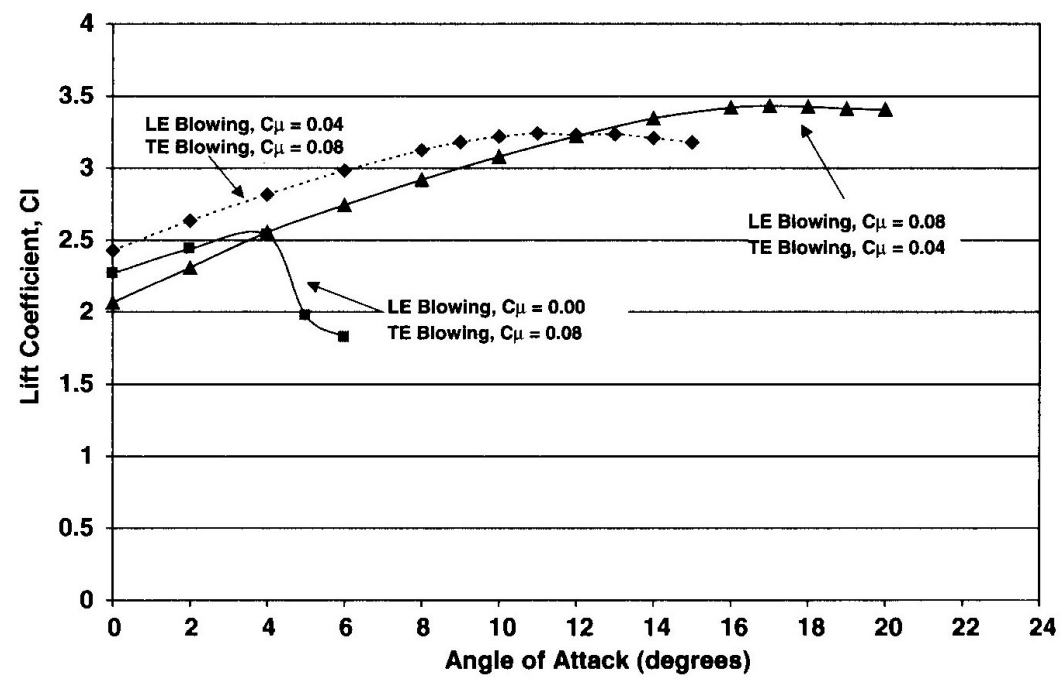

Figure 1-10: Mostly Linear Relationship between Angle of Attack and Lift ${ }^{16}$

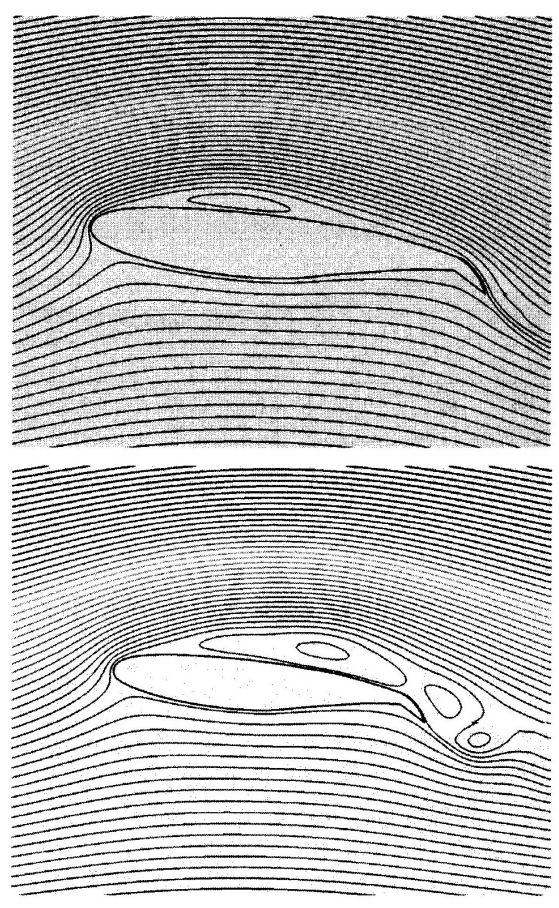

Figure 1-11: Stall due to Separation at the Leading Edge ${ }^{16}$

Another study ${ }^{17}$ specifically compared the results of CFD using Fluent to results obtained using the FUN2D solver and experimental results ${ }^{18}$. A coupled-implicit solver was used with second order upwind node-based discretization for the flowfield and 
turbulence equations. The Spalart-Allmaras turbulence model was used in order to best match the previously performed CFD results ${ }^{18}$. The General Aviation Circulation Control (GACC) airfoil was used for this study. A simple sweep was performed of a two dimensional design space which included angle of attack and blowing coefficient $\left(\mathrm{C}_{\mu}\right)$. Fifteen different cases were run with at five blowing coefficients and three flap deflections. These results can be seen in Figure 1-12 from reference 17.

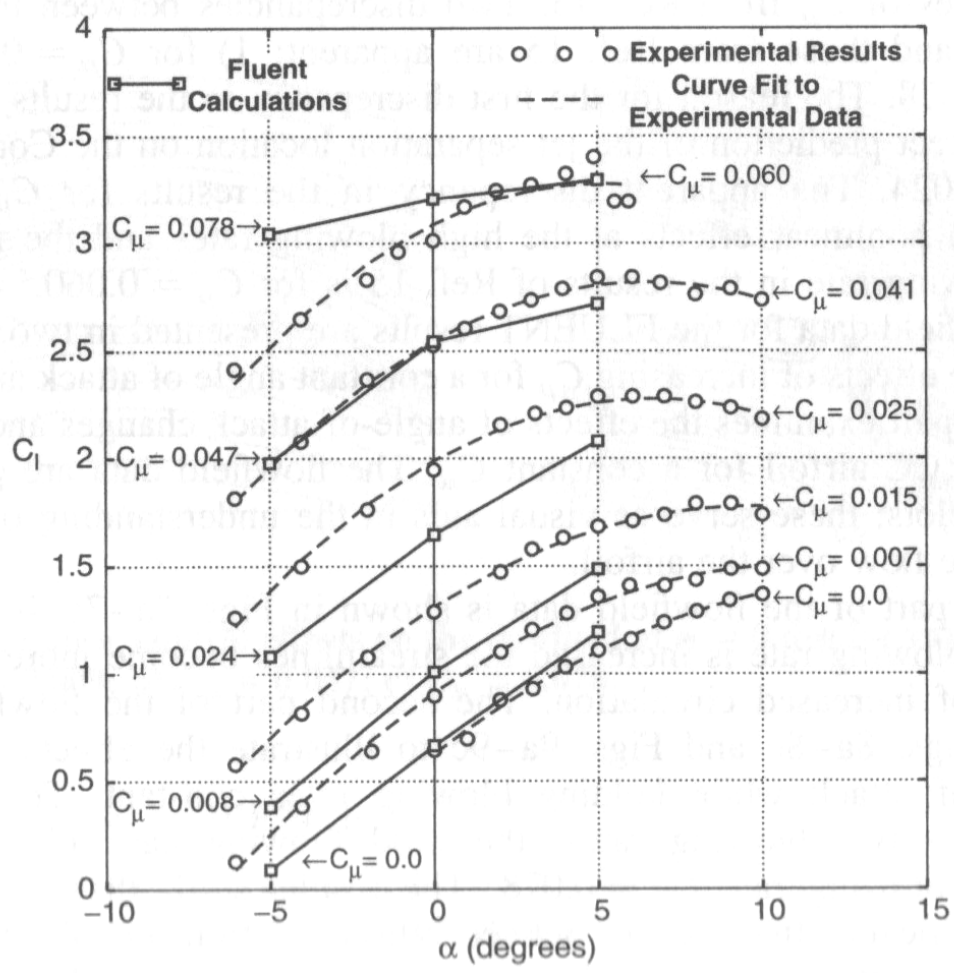

Figure 1-12: Fluent Results Compared to Experimental ${ }^{17}$

These results show that at some blowing coefficients Fluent predicted the $\mathrm{C}_{1}$ better than others. The only two cases where there were large discrepancies from the experimental data were the blowing coefficients of 0.024 and 0.078 . The paper explains that for the lower of the two, the separation point on the trailing edge was poorly 
predicted and caused the poor $\mathrm{C}_{1}$ results. For the higher blowing coefficient, the problem is attributed to the non-linear effects at high blowing rates. Several flowfields from this study at zero degrees angle of attack can be seen in Figure 1-13 from reference 17. The left pictures from the figure show how drastically the stagnation points shift at increasing blowing coefficients while the pictures on the right depict the growing recirculation region behind the trailing edge.
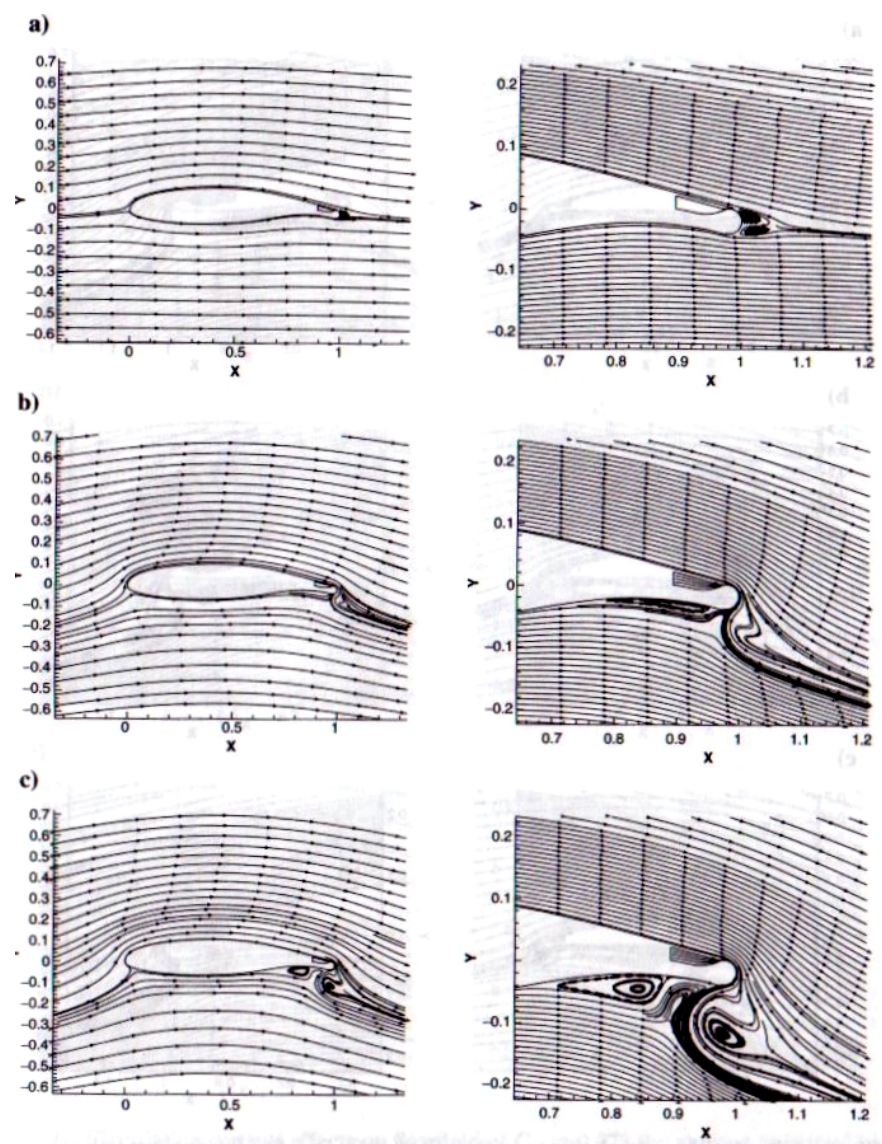

Figure 1-13: Flowfield at $\alpha$ of 0 and $C_{\mu}$ of a) 0.000 b) 0.047 c) $0.078^{17}$

Another study ${ }^{19}$ showed the specific use of two turbulence models, k- $\omega$ and k- $\zeta$, as applied to the $103 \mathrm{RE}(103 \mathrm{XW})$ airfoil which was tested at transonic speeds experimentally by Abramson and Rogers ${ }^{20}$. Although only the two previously mentioned turbulence models were used in this study, the Spalart-Allmaras model, 
specifically the version which includes curvature effects (SARC), was said to be the only model used in the 2004 NASA/ONR workshop that "performed well for all jet momentum coefficients."19 Regardless of this fact, the two previously mentioned turbulence models were used because of their abilities to correctly predict wall-bounded shear flows and the growth of all free shear layers including jets, wakes, and mixing layers. The numerical solutions for this study were performed using CFL3D with grids of varying density which had as many as 70,563 cells. The results of this study showed reasonably good predictions from both turbulence models with a slightly better performance out of the k- $\zeta$ model. These results can be seen in Figure 1-14 from ref. 19.

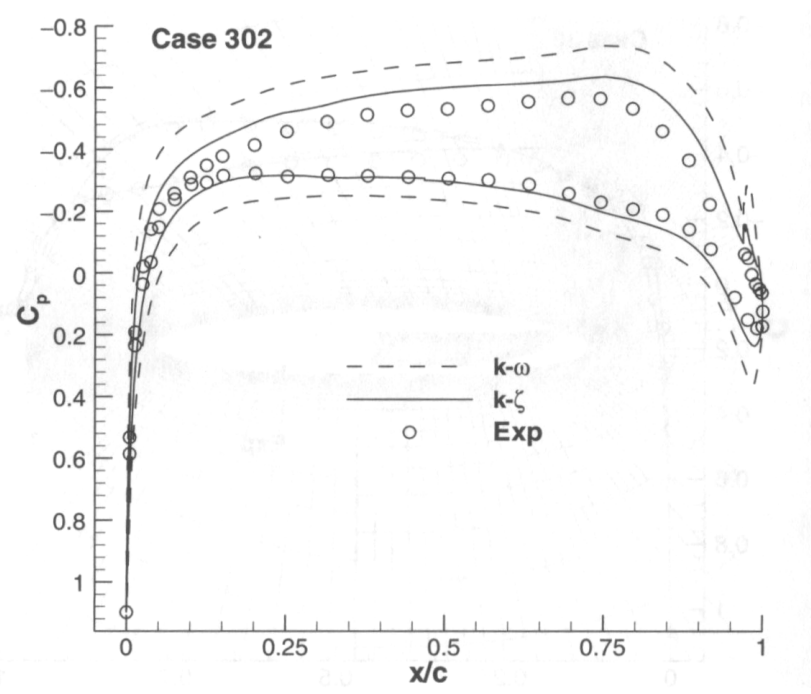

Figure 1-14: Comparison of $C_{p}$ using $k-\zeta$ and $k-\omega$ for $C \mu$ of $0.0032^{19}$

From the figure it can be seen that both models over predicted $C_{p}$ values from the experimental results with the k- $\omega$ model with the slightly worse prediction. The reason for the higher lift predicted by the $\mathrm{k}-\omega$ model was explained as a result of a later prediction of the separation point from the Coanda surface of the high speed jet flow which can be seen in Figure 1-15 from ref.19. 

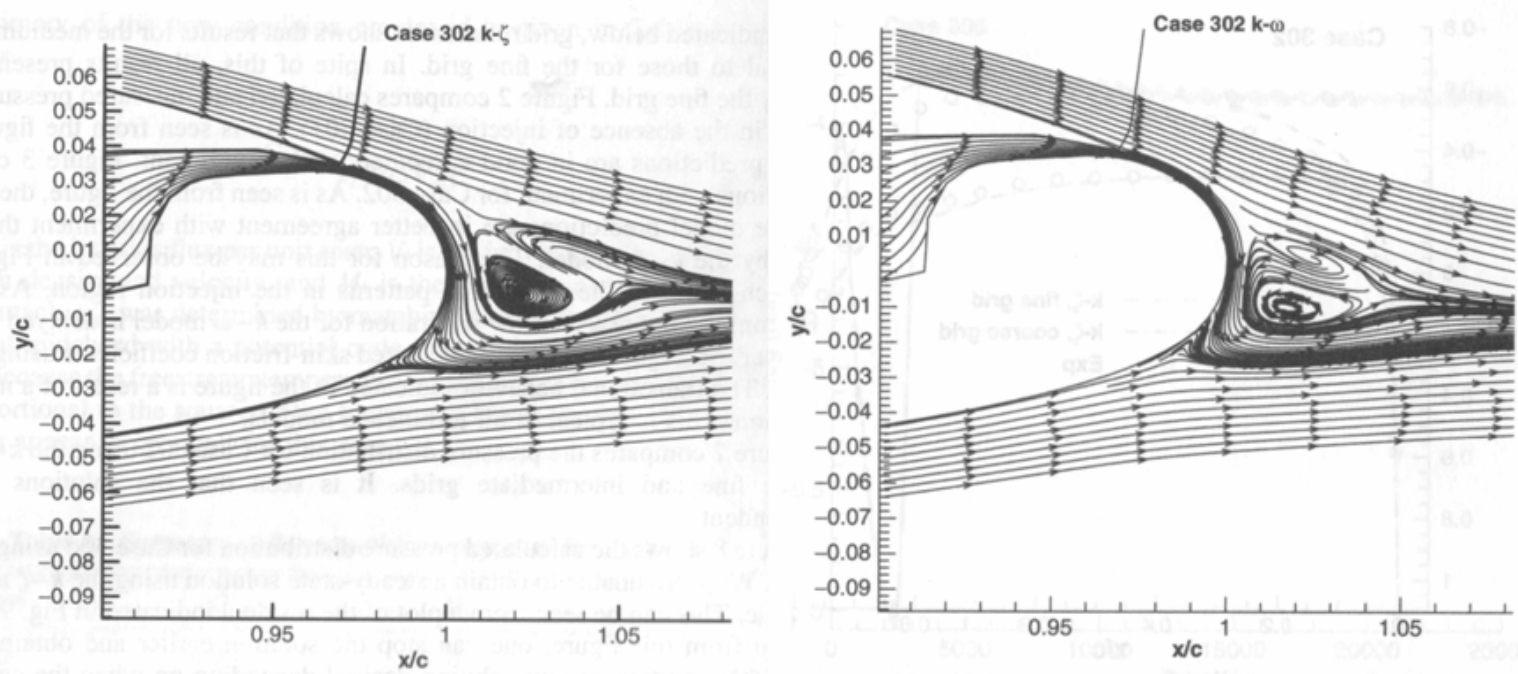

Figure 1-15: Coanda Surface Streamlines for $C \mu$ of 0.0032 of left: $k-\zeta$ and right: $k-\omega^{19}$

Additional results from this study showed how the user could specify laminar, transitional, or turbulent flow for different blocks within CFL3D. By specifying the jet flow as fully laminar and fully turbulent, entirely different separation points and recirculation regions were predicted as can be seen in Figure 1-16 from ref. 19. The study suggests that the reason high blowing coefficients are not predicted well by turbulence models is because the flow is not fully turbulent throughout and would be modeled better by use of transitional flows.
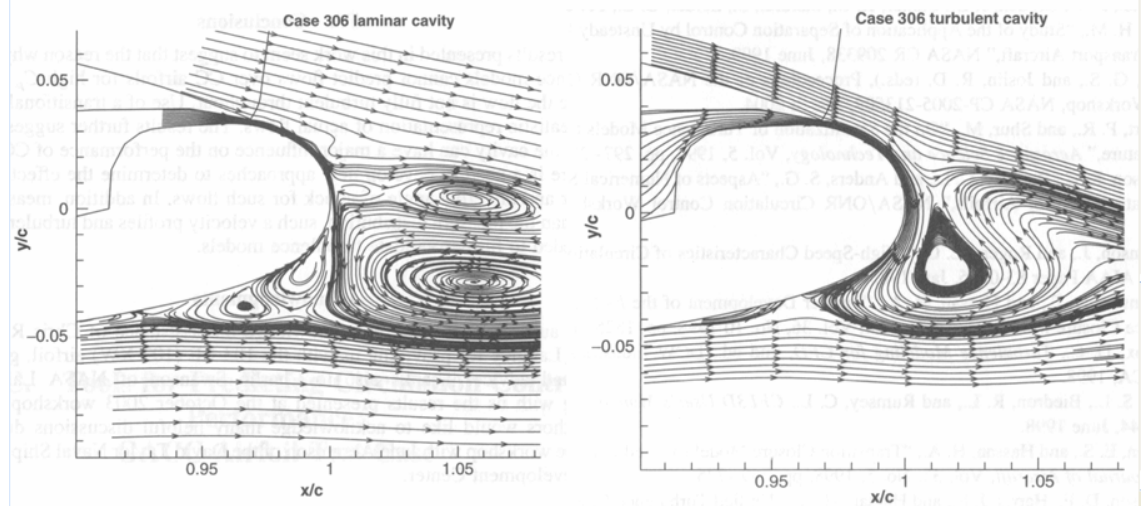

Figure 1-16: Differing Flow Results for left: Laminar and right: Turbulent jet flows ${ }^{19}$ 
One of the only studies which to date has performed three-dimensional CFD for a CC wing was that of ref. 21. The goals of that research were to extend the knowledge base of how specific turbulence models, namely k- $\zeta$ and Menter's shear stress transport (SST), performed on a three dimensional wing and to compare that to wind tunnel testing in an effort to bring the technology to more aircraft. It has been well established that CFD solutions of CC "depend greatly on turbulence models, grid densities, and numerical parameters. ${ }^{, 21}$ This research used what the authors deemed capable turbulence models for $\mathrm{CC}$ as well as testing for grid dependence in order to develop accurate performance predictions for their 3-D wing at two blowing coefficients: $C_{\mu}=0.03$ and $C_{\mu}=0.1$. Aside from the overall lift coefficient, the major comparisons between the computational and experimental results included $\mathrm{C}_{\mathrm{P}}$ distributions, separation points, and selected velocity profiles. At the lower blowing coefficient, it was found that the $k-\zeta$ model did a reasonable job predicting the flow characteristics with the $\mathrm{C}_{\mathrm{P}}$ distribution mostly matching that of the experimental run as can be seen in Figure 1-17 from reference 21. A slight over prediction of the pressure at the suction peak resulted in a slightly higher lift prediction over the experimental results.

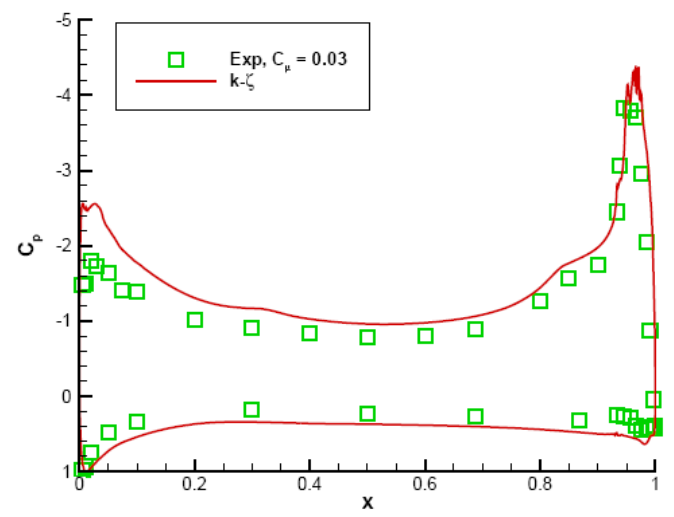

Figure 1-17: $C_{p}$ Distribution over CC Wing at $C_{\mu}$ of 0.03 using $k-\zeta^{21}$ 
The velocity profiles at various angles around the Coanda surface were shown to agree at most angles except for those near the separation point. The discrepancy may have been caused because the actual separation point was predicted to occur at a higher angle than was shown experimentally. Although the lower mass flow case tended to mostly agree with the experimental values, the higher mass flow case was not as agreeable. Both of the turbulence models used for this case predicted reasonable $C_{P}$ distributions up to about $80 \%$ of the lower surface but neither was able to accurately predict the upper surface. The more interesting result was the prediction of the separation point on the lower surface at $80 \%$ and $75 \%$ chord for the $\mathrm{k}-\zeta$ and SST models respectively which can be seen in Figure 1-18 from reference 21. In an attempt to show grid independence, three different grids were generated. It was shown that the results in the form of $\mathrm{C}_{\mathrm{P}}$ distribution more closely matched the experimental results with the grid with the more refined region near the jet slot.
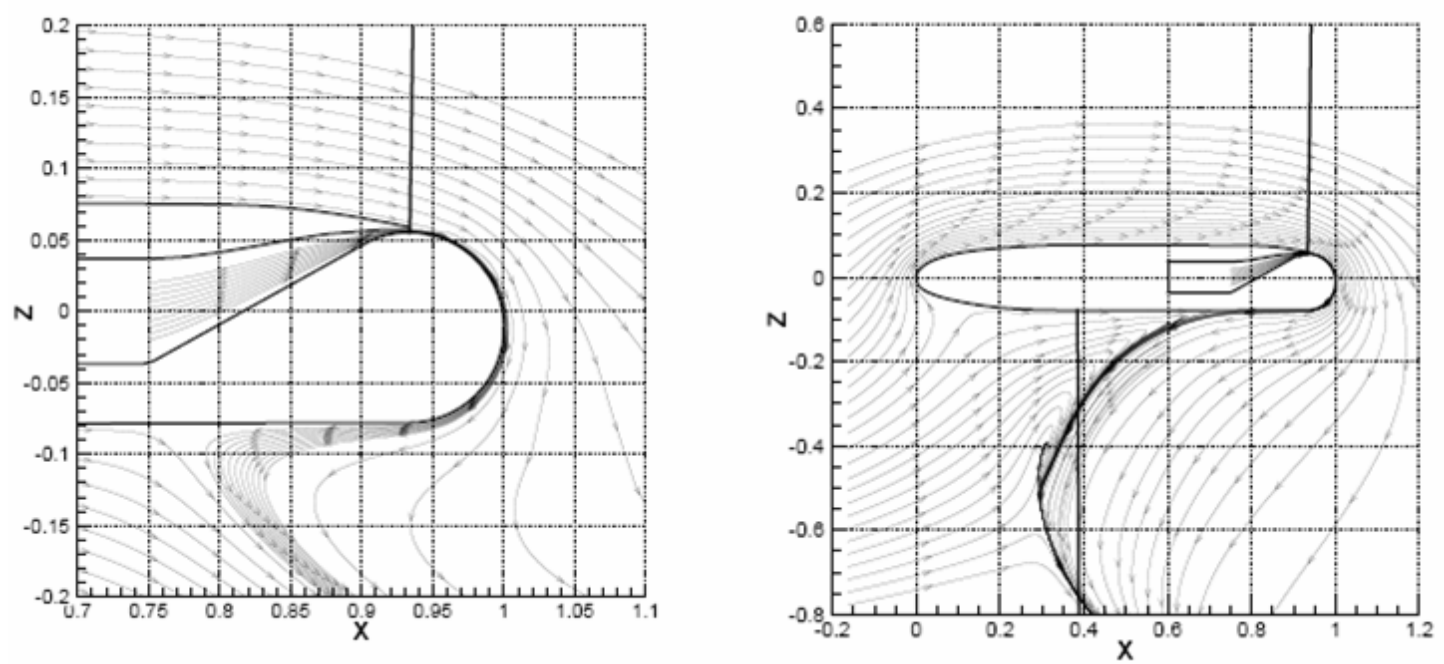

Figure 1-18: Non-Physical Separation at $C_{\mu}$ of 0.1 using left: $k-\zeta$ and right: $k-\omega S S T{ }^{21}$ 
Many other papers have been published on circulation control and have investigated different airfoil designs and uses of turbulence models and their applications. However, the preceding summary was deemed sufficient and for further detail, please consult the sited references. 


\section{Research of de la Montoya}

Since this study was a continuation of previous research ${ }^{13}$, it is important to show the previous results as well as some of the details which have carried over to the current research. Specifically, the geometry of the circulation control wing and its origins will be explained as well as the final results of de la Montoya ${ }^{13}$.

\subsection{Circulation Control Wing Geometry}

The basic CC wing geometry for this study was based the Model 114 ESTOL vehicle concept created by the California Polytechnic State University, San Luis Obispo in conjunction with NASA Ames ESTOL sector as an ESTOL study reference vehicle during the summer of $2004^{22}$, the final configuration which can be seen in Figure 2-1 from reference 13 . The airfoil for the $\mathrm{CC}$ wing used the previously mentioned GTRI dual radius flap with the airfoil based on the NASA SC(2)-0414.

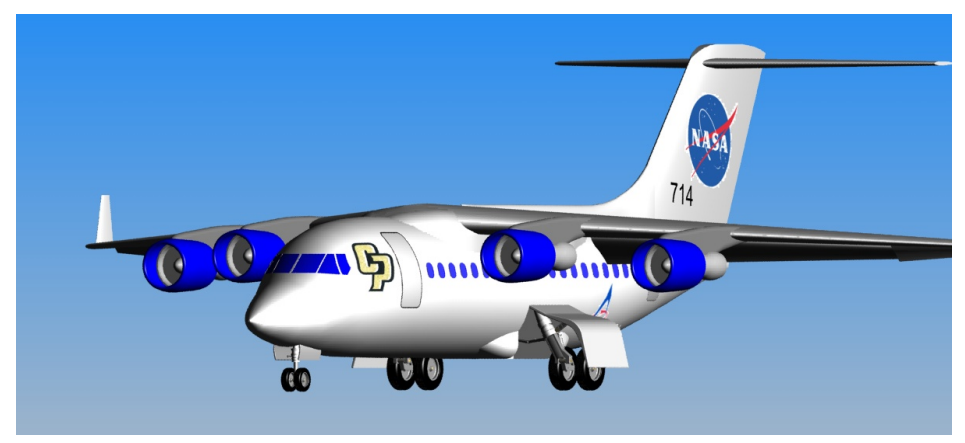

Figure 2-1: Model 114 ESTOL Concept ${ }^{22}$

A dual radius flap located at roughly $0.9 \mathrm{c}$ was added to that airfoil and given a slot height to chord ratio of 0.0016 which is below the 0.002 upper limit where the jet flow then becomes inefficient as was previously discussed ${ }^{14}$. Due to symmetry, only half of the wing was required for the CFD model. The half wing, as seen in Figure 2-2 had a reference area of $36.153 \mathrm{~m}^{2}$ with $9.63^{\circ}$ of leading edge sweep and a dual radius flap 
which extended $80 \%$ of the span with an area of $0.04459 \mathrm{~m}^{2}$. A detail of the dual radius flap can also be seen in Figure 2-2. The inboard section of the wing had a 1.829 meter section without the dual radius flap to account for the region of the wing which would intersect the fuselage.
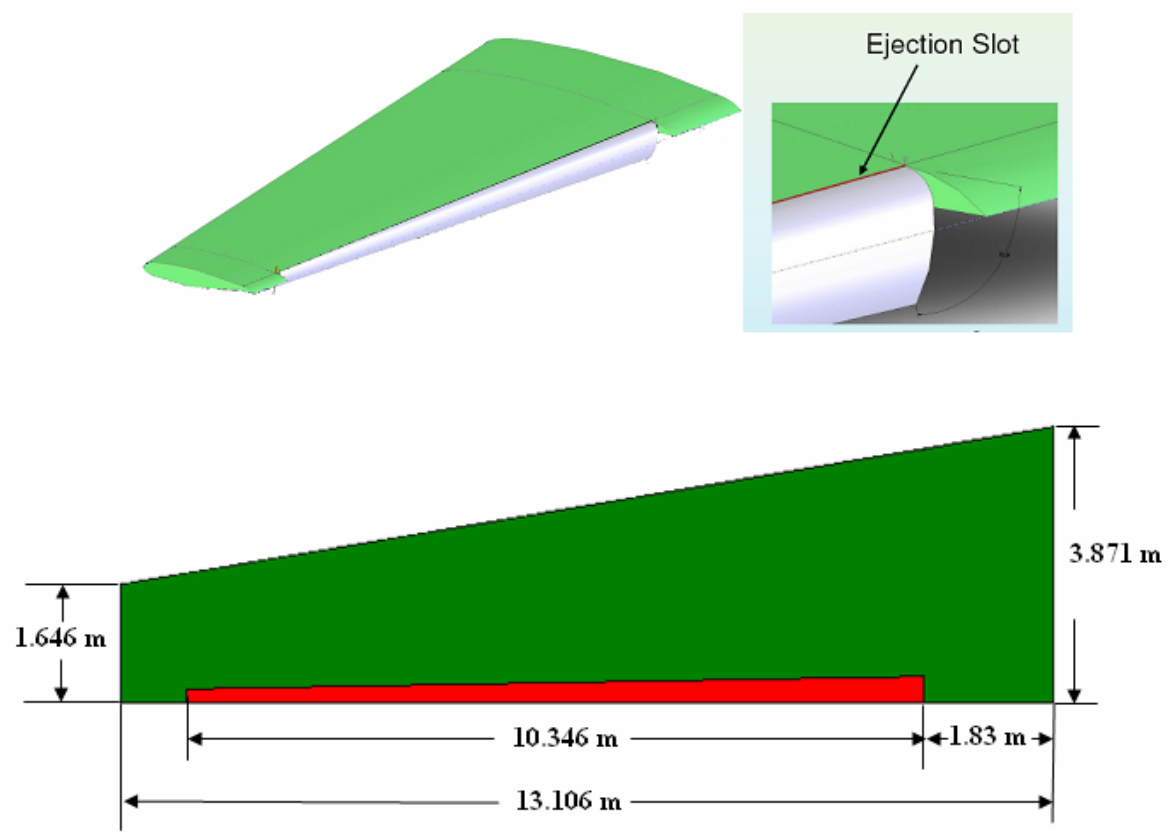

Figure 2-2: Circulation Control Wing and Ejection Slot

\section{$2.2 \quad$ Results}

One of the primary goals of this research was to improve upon the results previously obtained by de la Montoya ${ }^{13}$. A brief summary of those results will be included here so that comparisons can be made.

\subsubsection{Grid Dependence}

In order to determine whether or not grid independence was achieved, Montoya performed several tests with different types of grid adaption within Fluent. Two different flap deflections, zero and $90^{\circ}$, were tested with no adaption and those results were 
compared to cases of pressure adaption and shear stress adaption. It was found that the largest discrepancy was a $6.2 \%$ decrease in $C_{D}$ from the non-adaption to the pressure adaption cases. In addition, it was mentioned that a $\mathrm{y}^{+}$investigation was undertaken and it was found that the boundary layer may not have been resolved near the jet slot. Grid dependence for the current study addresses both the issues of grid density and boundary layer resolution and will be discussed in section 8.2.

\subsubsection{Turbulence Model Validation}

In order to determine which turbulence model would be used in her study, Montoya tested the Spalart-Allmaras model against 2D wind tunnel test results provided by Georgia Tech Research Institute (GTRI). These results can be seen in Figure 2-3 with GTRI's wind tunnel and computational results. The green data points clearly show that Montoya achieved results which agreed very well with those from GTRI.

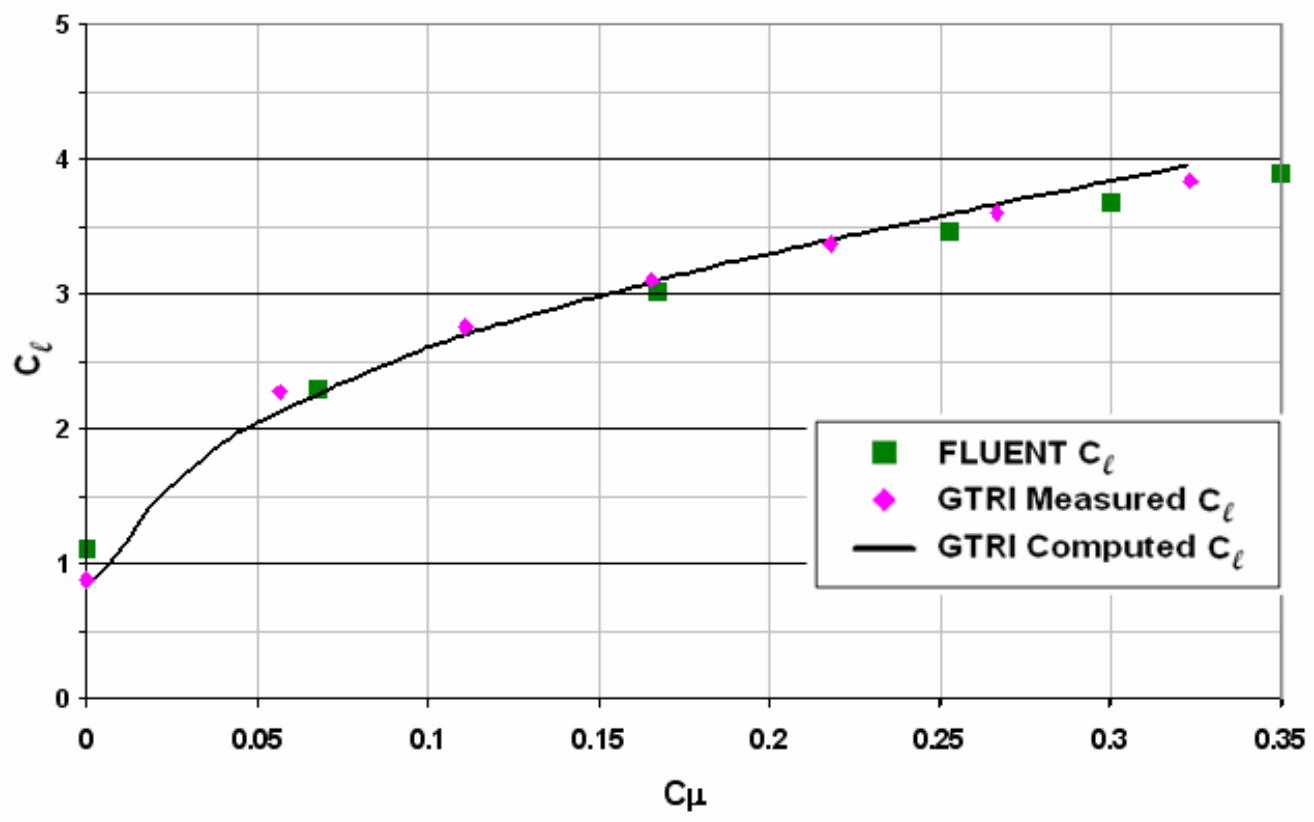

Figure 2-3: Montoya's Spalart-Allmaras Validation $^{13}$ 
This same type of validation was performed for the current study with additional comparisons with the k- $\omega$ turbulence model as well as a grid density comparison.

\subsubsection{Three-Dimensional Analysis}

To understand how the three-dimensional CCW performed, Montoya performed a full factorial sweep over design variables which included angle of attack, blowing coefficient, and four flap deflections: zero, 30,60 , and $90^{\circ}$. This amounted to about 120 3-D cases that were run to define the design space. A plot of how the lift varied with angle of attack and blowing coefficient with the $60^{\circ}$ flap can be seen in Figure $2-4^{13}$.

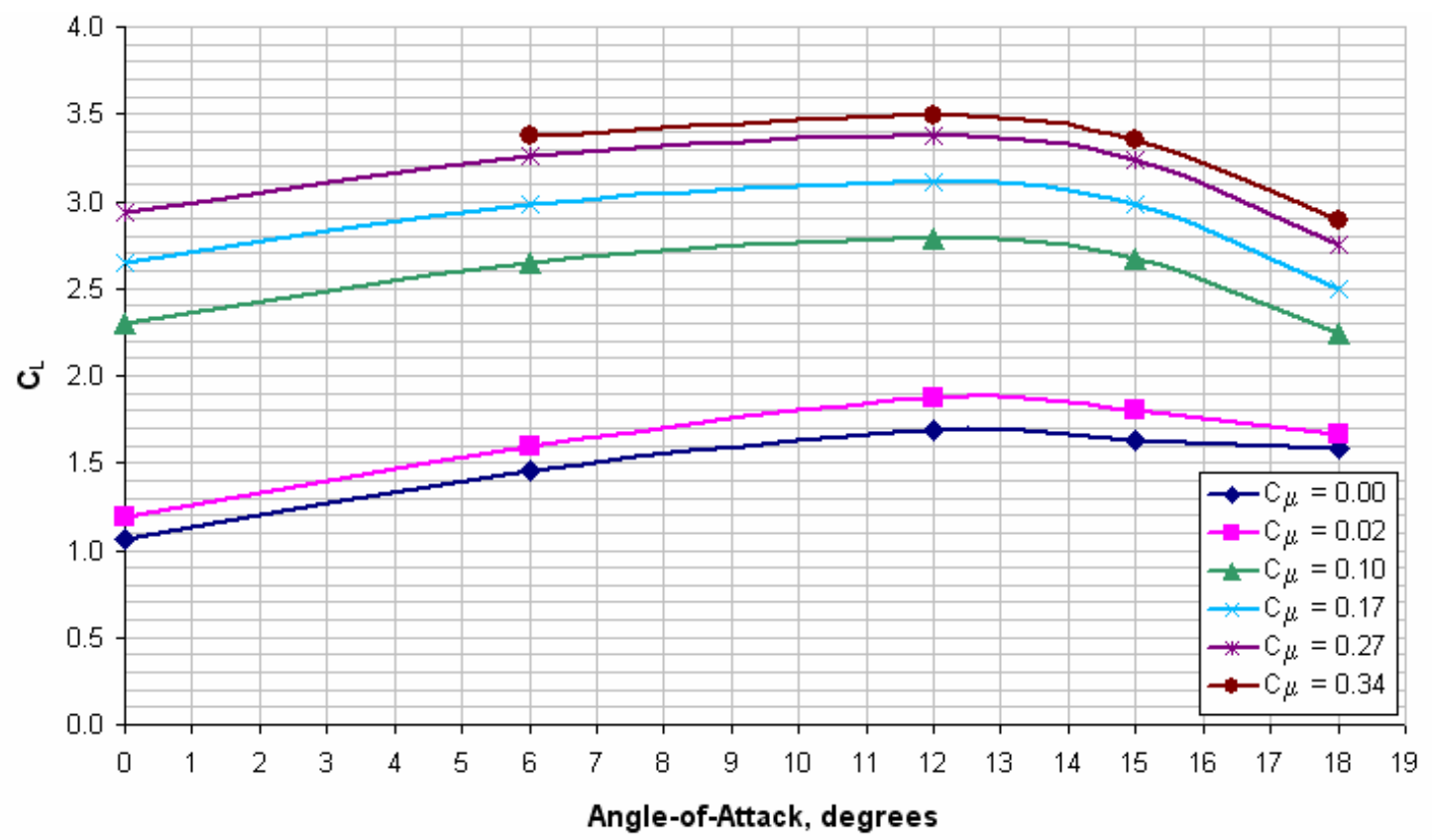

Figure 2-4: Lift Variation vs. Angle of Attack at Various Blowing Coefficients ${ }^{13}$

Several more similar plots were produced at the different flap deflections as well as the corresponding drag polars. This information was later used to calculate balanced field length calculations using an equation very similar to $\mathrm{Eq}(1)$. Some of the results of that analysis for a flap deflection of $60^{\circ}$ can be seen in Figure 2-5 from ref. 13. These results show how the BFL was reduced as the blowing coefficient was increased as well as the 
angle of attack. Although informative, this analysis was improved upon in several areas in the current research. These improvements will be summarized here but discussed in detail in later sections. First, instead of using the BFL equation found in $\mathrm{Eq}(1)$ for reasons already cited, the equations of motion were integrated throughout the takeoff for more accurate results.

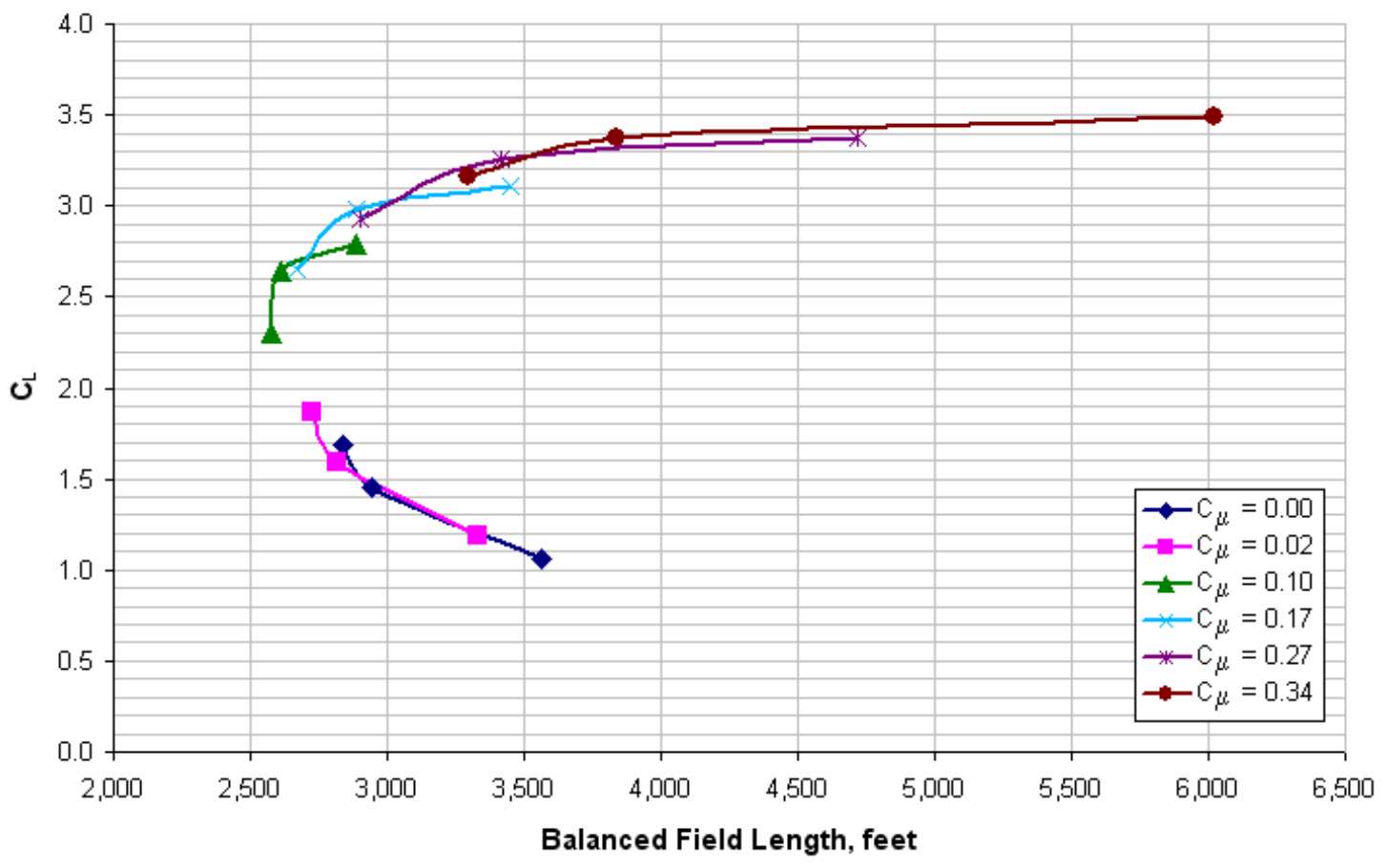

Figure 2-5: Montoya's BFL Calculations for a $60^{\circ}$ Flap $^{13}$

Second, a fourth design variable of freestream Mach number was added to incorporate its effect throughout the takeoff run. Third, and most significant, the number of flap deflections and blowing coefficients was increased significantly in order to determine optimal performance of the CCW during takeoff. With the use of Gaussian Processes to create a metamodel of the design space, all of this was achieved with only a third the number of CFD cases. 


\section{Turbulence Model Validation}

As with Montoya's research, it was important to verify which turbulence model would accurately predict the flow phenomenon produced by circulation control. As has been previously discussed, references 16, 17, and 19 all performed CFD using the Baldwin-Lomax, Spalart-Allmaras, k- $\omega$ SST, and k- $\zeta$ turbulence models. Because of availability and the good results previously shown for Spalart-Allmaras and k- $\omega$, these models were used in CFD cases in an attempt to match the same GTRI wind tunnel data which was previously presented.

\subsection{Spalart-Allmaras and $\mathrm{Y}^{+}$Analysis}

It was important to make sure the turbulence model behaved well at slow speeds in order for a takeoff run to be properly examined and also to correctly resolve the shear layer at the jet slot. In order to accomplish this, multiple two-dimensional grids were created around the dual radius circulation control airfoil which were then solved under the same conditions as those found in ref. 23 which contained published wind tunnel data. These two grids had maximum $\mathrm{y}^{+}$values of about 50 and 500 and are thus called the fine and coarse grids. The fine grid was solved using both turbulence models while the coarse grid only with Spalart-Allmaras. The lift as a function of blowing coefficient for the different grids and turbulence models can be seen in Figure 3-1. The coarseness of the grid and the type of turbulence model clearly has a significant impact on the results. The solution that most agrees with the experimental data utilized the coarse grid with the Spalart-Allmaras turbulence model. A possible reason for the significant difference between the Spalart-Allmaras results could be due to too much refinement in the fine grid. 


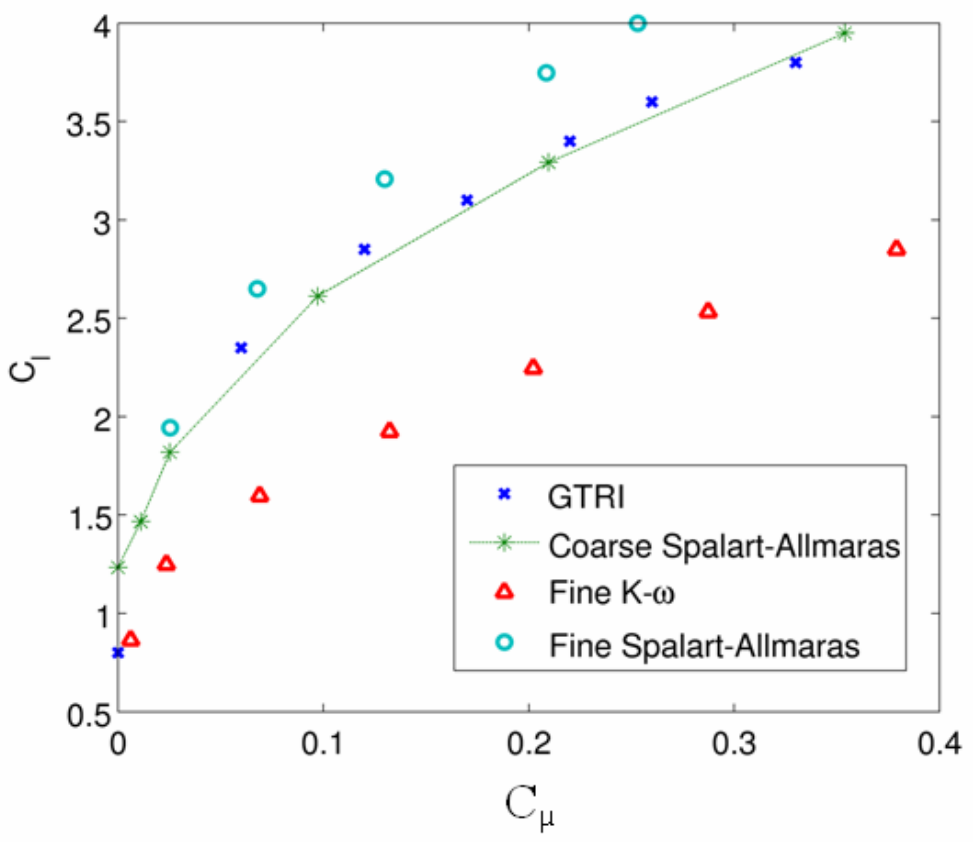

Figure 3-1: Solver and Grid Comparison

Since it had a maximum $y+$ of 50 , than a significant portion of the airfoil had lower values which put those boundary layer cells within the buffer region and laminar sublayer which in turn could have introduced some error. At any rate, the SpalartAllmaras model proved to be the better of the two as long as the $\mathrm{y}^{+}$was not within the lower boundary layer regions.

\subsection{Turbulence Model Separation Verification}

Flow separation is very difficult to predict accurately using CFD. With the higher dual radius flap deflections, it was found that a significant recirculation region existed directly aft of the flap. For more details as to how this separation was discovered, see section 6.5. In order to validate how well SA predicted the separation for a $2 \mathrm{D}$ case, an additional grid and case was generated and run using the k- $\varepsilon$ and k- $\omega$ SST turbulence models which have the option to use "enhanced wall treatment". With this option enabled, the viscosity-affected near-wall region is completely resolved all the way into 
the viscous sublayer ${ }^{24}$. This analysis was performed in only two dimensions to speed computation and to more closely analyze the separation.

Before this analysis could take place, a two-dimensional grid with a $\mathrm{y}^{+}$within the viscous sublayer $\left(\mathrm{y}^{+} \sim 1\right)$ needed to be generated. The low $\mathrm{y}^{+}$requirement necessitated the use of boundary layer cells over the entire airfoil surface because tetrahedrals could not be generated efficiently and small enough to achieve the desired $y^{+}$. The final grid with the boundary layer cells can be seen in Figure 3-2.
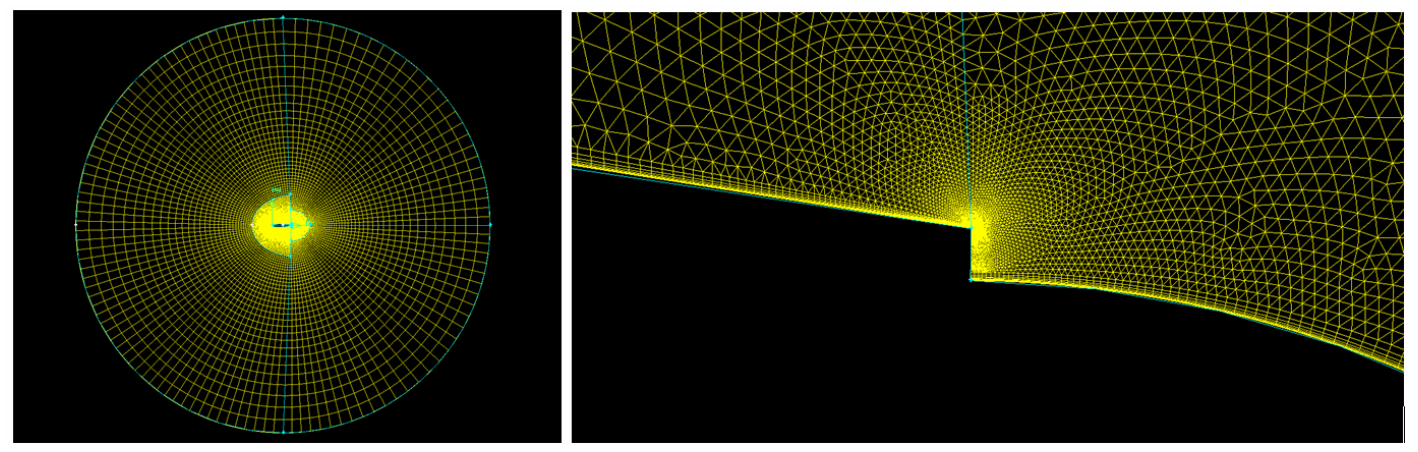

Figure 3-2: Fine Grid with $\mathrm{Y}^{+}$near 1 using Boundary Layer Cells

This grid was meshed using a technique that utilized triangular cells in the small region surrounding the airfoil and quadrilateral cells which extend to the outer boundaries. This technique provides fewer triangular cells which in turn reduces the amount of interpolation required between adjacent cells. With the refined grid generated with the required $\mathrm{y}^{+}$, the test case was then solved using $\mathrm{k}-\varepsilon$ with the enhanced wall functions enabled. The results of this case solved to the first order can be seen in Figure 3-3. 


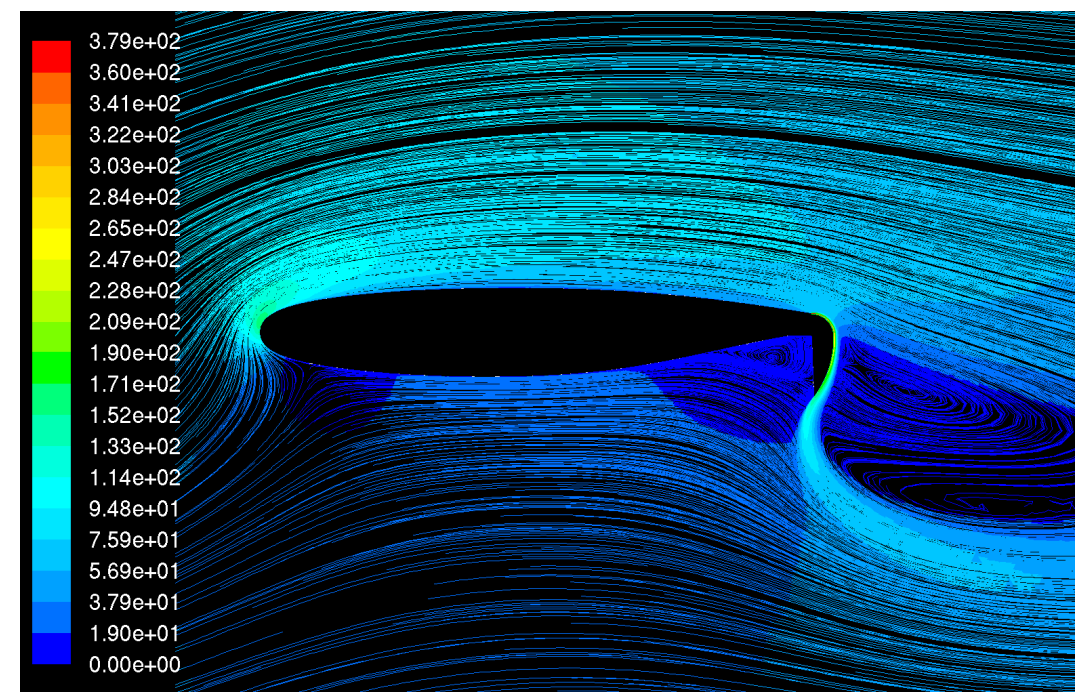

Figure 3-3: K-ع Solution with Enhanced Wall Treatment (velocity streamlines m/s)

The results can be seen to be very similar to those from the coarser SA solution which is shown in Figure 6-7. A second order solution was attempted but a steady state convergence was never obtained. Although the SA results using the coarse grid appeared very similar to those of $k-\varepsilon$, a steady state solution for the SA case using wall functions was never achieved. However, unlike the second order k- $\varepsilon$ case, the residuals showed periodic behavior, as seen in Figure 3-4, which indicated vortex shedding in the flowfield.

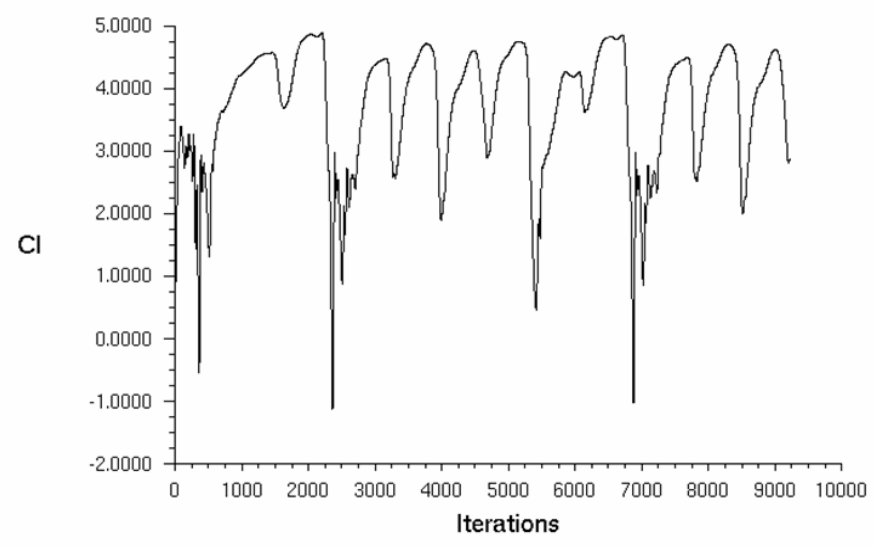

Figure 3-4: Periodic Behavior Due to Vortex Shedding with Coarse Grid 
The oscillations for this case were caused by vortices being shed from the upper surface of the airfoil which occurred because of the $7.9^{\circ}$ angle of attack coupled with the supercirculation and high flap deflection. This periodic behavior can be seen in Figure 3-5.

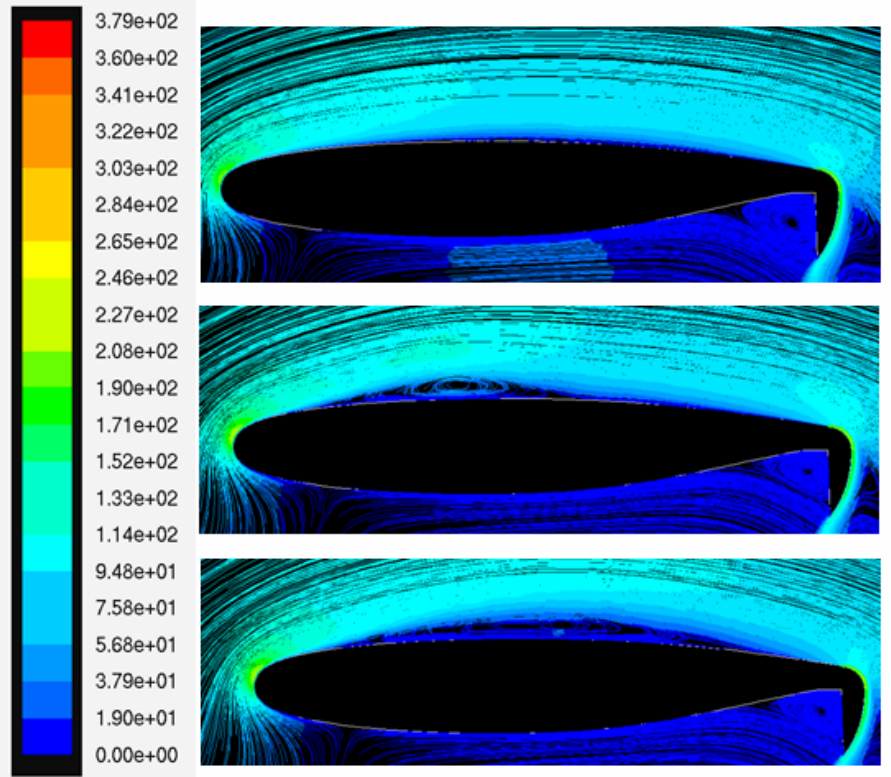

Figure 3-5: Vortex Shedding in Coarse SA Solution (velocity streamlines, m/s)

Although the coarse grid with SA resulted in unsteady values for $C_{l}, C_{d}$, and $C_{m}$, the average result actually compared reasonably well when compared to the two-equation models as can be seen in Table 3-1.

Table 3-1: Turbulence Model Results for Separated Case

\begin{tabular}{|c|c|c|c|}
\hline Turbulence Model & $\mathrm{C}_{\mathrm{l}}$ & $\mathrm{C}_{\mathrm{d}}$ & $\mathrm{C}_{\mathrm{m}}$ \\
\hline Spalart-Allmaras $\left(\mathrm{y}^{+} \sim 312\right)$ & $3.5 \pm 1$ & $0.3 \pm 0.1$ & $0.55 \pm 0.25$ \\
\hline $\mathrm{k}-\varepsilon\left(\mathrm{y}^{+} \sim 2\right)$ & $3.24 \pm 0.01$ & $0.3755 \pm 0.001$ & $0.955 \pm 0.005$ \\
\hline $\mathrm{k}-\omega \operatorname{SST}\left(\mathrm{y}^{+} \sim 2\right)$ & $2.9 \pm .02$ & $0.37 \pm .015$ & $0.83 \pm 0.02$ \\
\hline
\end{tabular}


Even though the results for the coarse grid were more unsteady than the others, the average integrated values actually compared relatively well. This argument supports the use of the coarse 3D grids using SA. Even though the exact flow features throughout the grid and wake were not captured perfectly, the integrated values were able to be obtained with a minimum of computational effort and could provide reasonable preliminary solutions for separation which could then be used in the takeoff performance model.

Although the flow features on the upper surface of the airfoil were not resolved completely with the coarse grid, it can be seen from Figure 6-7 and Figure 3-3 that the separation region behind the jet slot was largely independent of the vortex shedding on the upper surface. With this independence established, the next comparison between the models was the separation point of the freestream flow from the CC flap. Traditional separation can be located quite accurately by examining the $\mathrm{C}_{\mathrm{p}}$ on the airfoil surface. This technique does not apply well to the CC flap due to the high speed mass flow which remains completely attached to the flap. Instead, a graphical approach was used to estimate the angle at which the freestream flow detaches from the high speed ejected flow. The angle was measured from the inner flap corner for the three cases and is pictured in Figure 3-6. 


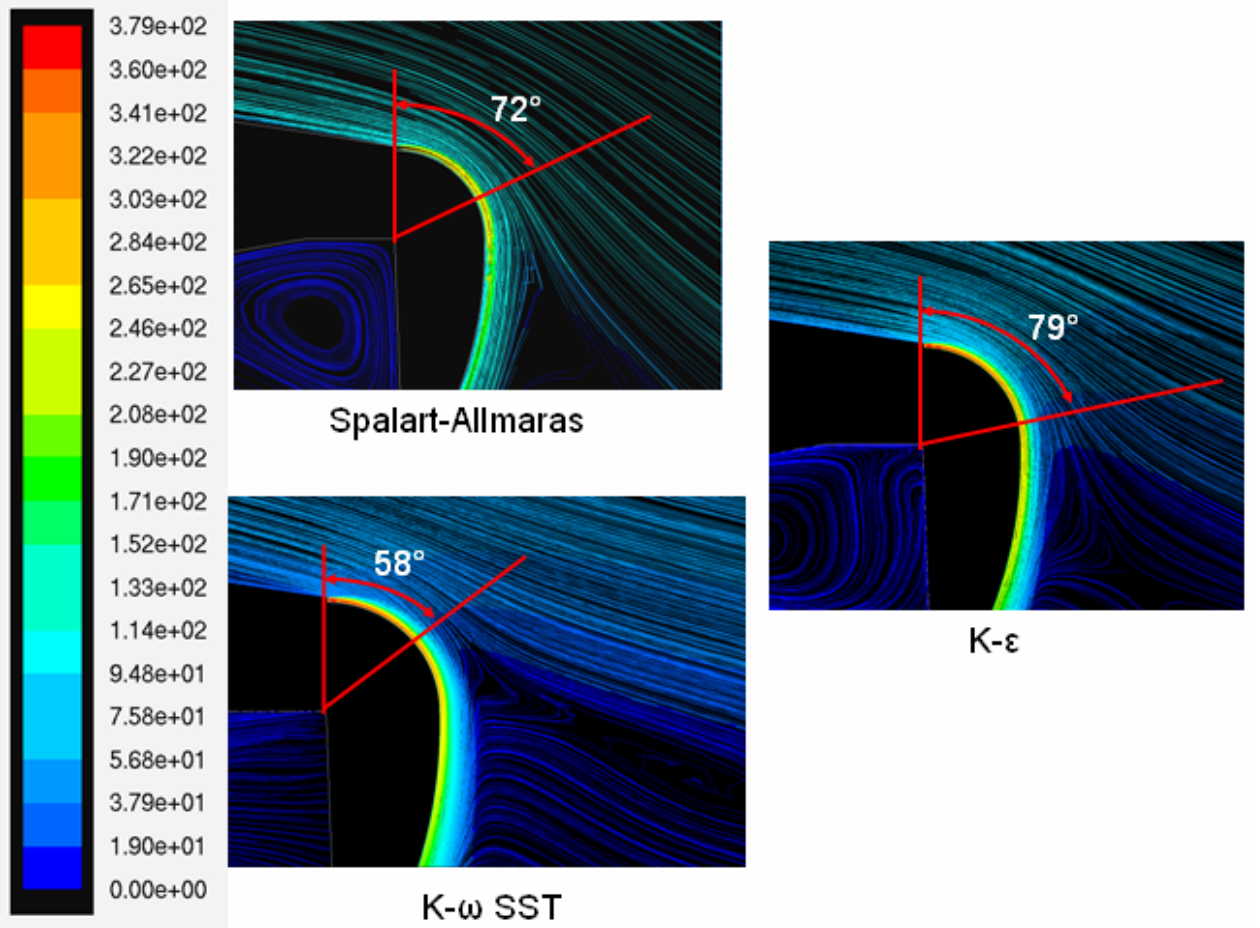

Figure 3-6: Trailing Edge Separation Comparison (velocity streamlines, $\mathbf{m} / \mathbf{s}$ )

From this figure, the coarse SA grid utilizing wall functions predicted the separation point about $7^{\circ}$ sooner than the k- $\varepsilon$ model and $14^{\circ}$ later than the k- $\omega$ SST model. Again, although the wall function approach did not capture the exact flow phenomenon found using the near wall two equation models, the results show that the Spalart-Allmaras model appears to provide comparable values for $\mathrm{C}_{\mathrm{L}}, \mathrm{C}_{\mathrm{D}}$, and $\mathrm{C}_{\mathrm{M}}$ as well as separation modeling. In addition, the two equation models have a $19^{\circ}$ difference in their separation points and without experimental data to validate against, it is difficult to say that the two equation models are predicting separation any better than the wall function approach. 


\section{Design of Experiments}

One of the major drawbacks for the current simplified methods for calculating takeoff distance is their lack of knowledge of how the lift and drag of an airplane vary throughout the takeoff run. Although the simplified models provide adequate results for traditional aircraft, the results for STOL aircraft are largely inadequate. This inadequacy is due to the fact that there are no analytical methods to accurately calculate the lift and drag of an aircraft using powered lift such as circulation control. A previous solution to this problem by Percey and Margason ${ }^{25}$ used available wind tunnel test data and developed a quadratic curve fit relationship between $\mathrm{C}_{\mathrm{Lmax}}$ and the blowing coefficient for USB. This technique of creating quadratic curve fits to wind-tunnel data was repeated several times throughout their paper in order to establish relationships of how lift, drag, and thrust vary during takeoff.

A similar but higher fidelity approach was used in this study, where CFD was performed on the previously described $\mathrm{CC}$ wing over a variety of Mach numbers, angles of attack, flap deflections, and mass flow rates. Instead of performing a computationally expensive full factorial experiment on the four design variables, a Monte Carlo approach was used to define the design space with a minimum number of CFD cases. For Mach number, angle of attack, and mass flow rate, the test points were satisfied the Monte Carlo by being purely random throughout each of their ranges: Mach between 0.06 and 0.35 , angle of attack between $-2^{\circ}$ and $8^{\circ}$, and mass flow rate between 0 and $20 \mathrm{~kg} / \mathrm{s}$. It would have been desirable to obtain solutions at even lower Mach numbers but for cases run at Mach numbers much less than 0.06, the solutions tended to become unstable and rarely converged. The purely random Monte Carlo had to be modified for the flap 
deflection because each deflection required a unique grid. It was assumed that the flap deflection behavior would be relatively stable with no irregular jumps or steps which allowed for the random flap deflections to be rounded to the nearest three degrees between zero and $90^{\circ}$ without compromising the benefits of the Monte Carlo. This discritization allowed for a maximum of 30 three-dimensional grids rather than a unique grid for every test case.

One of the common parameters that is used in $\mathrm{CC}$ performance is $\mathrm{C}_{\mu}$ or blowing coefficient which was defined by $\operatorname{Eq}(2)$. The blowing coefficient cannot be determined before a case is run due to the unknown state at the ejection slot. As a result, the Monte Carlo for the current study was performed over mass flow rate instead of blowing coefficient. However, the average jet velocity was recorded for each converged solution in order to retroactively calculate the $\mathrm{C}_{\mu}$ for each case so that its effects on lift and drag could be modeled with the Gaussian processes. It turned out that generating random points throughout the range of zero to $20 \mathrm{~kg} / \mathrm{s}$ for mass flow rate did not adequately define an equivalent range of blowing coefficients. This was due mainly to the coupled relationship between $\mathrm{C}_{\mu}$ and the freestream conditions. Because of this coupling, not enough data points were obtained to create a model that was entirely accurate based blowing coefficients which were used in this study. To prevent this problem from occurring in the future, a method of bisection iterative routine was added to the Fluent automation script which attempts to predict the blowing coefficient at the start of a case using a regression of mass flow rate data in order to predict the final jet velocity. This regression can be seen as $\mathrm{Eq}(18)$. 


$$
V_{\text {jet }}=-.1791 \dot{m}^{2}+20.3253 \dot{m}-2.6022
$$

This method is limited to cases run with a jet exit temperature of $298.15 \mathrm{~K}$ because the regression was fit to CFD data only run with that specific temperature. The iterative scheme of predicting $\mathrm{C}_{\mu}$ was not meant to be $100 \%$ accurate at predicting the final blowing coefficient, but to provide a solution to the coupling problem. Even with the iterative scheme in place, the actual $C_{\mu}$ would have been calculated after the solutions converged.

Another advantage the Monte Carlo approach had over full factorial was the ability to provide interim results rather than requiring the entire design space to be populated with data points to produce a model. In the middle of the experiments, a model could be generated and provide some useful information about the system even if it was not fully resolved. As soon as additional data points stopped significantly changing the model generated by the Gaussian Processes (GP), the model was said to be accurate. More about how the GP worked and performed as well as its results will be shown in the next section. 


\section{Modeling and Simulation}

Although generating all of the CFD grids and solutions was all work performed by the author, a majority of the work in generating the metamodel for the CFD data and the writing and implementing of the BFL code was performed by his associate Scott Turner and therefore much of the following text which was taken from the author and $\mathrm{Mr}$. Turner's conference paper ${ }^{26}$ and is included in this section as a reference.

\subsection{Metamodeling and Gaussian Processes}

While modeling techniques attempt to predict the behavior of a system, metamodels attempt to predict the behavior of a model. This is useful for models that are expensive to obtain data points, such as CFD experiments, because relatively few points can be used to build a metamodel of the entire design region. It is impractical to calculate design points for the entire design space for CFD situations because takeoff calculations are based on a number of changing parameters, and changing any one parameter requires a new solution that will take many hours for the CCW case addressed. This effect was compounded when running an iterative BFL code, which was the final goal of the research. Thus the goal was to build a metamodel that captured all the important flow phenomena and was also quick to evaluate across the entire takeoff range.

The first component was to determine an experimental design for the collection of CCW CFD data points from which to build a model. A variety of model types were investigated including, full factorial, partial factorial, Latin hypercube, and full random. The factorial designs were eliminated because statistical analysis is not valid for an incomplete dataset, which limits the usefulness of the design and are typically reserved for experimental datasets with noise error. Classic DOE methods do not apply to 
computer experiments because for a given set of inputs, the outputs will always be the same, effectively eliminating the random error. The main source of error for such experiments comes from the bias error of the system, that is, the way in which the metamodel differs from the functional behavior of the model. The Latin hypercube and full random cases were examined and the Latin hypercube added no additional benefits while adding complexity since a fully random case was possible. Therefore, the final scheme was fully random for all of the design variables with the flap deflection discretized into three degree increments to cut down on gridding.

The response data was lift, drag and moment coefficients, which were taken from the Fluent cases. The factors that were varied per run were the Mach number, angle of attack, mass flow rate, and flap deflection. Each of those design variables was essential for determining the takeoff performance of an aircraft using circulation control. Varying the Mach number was necessary in order to evaluate the CCW performance throughout the takeoff process. Examining angle of attack provided information about when and how much the aircraft should rotate without stalling. The mass flow rate was a key parameter that could be used to size either the primary engines if bleed air was the source, or imbedded gas generators. And finally, as with the amount of air being ejected from the slot, the dual radius flap deflection had a significant impact on the amount of lift and drag produced by the aircraft and therefore was another important design feature to include in the metamodel.

The primary method used for creating the metamodel for this study was Gaussian process (GP). GP metamodels can be made to deal with noiseless data and the parameters used to define them can be optimized to fit the data. These capabilities were 
highly desirable for the CFD analysis of CCW aircraft. The GP models were based off of code written by Carl Rasmussen ${ }^{27}$ where the hyper-parameters are chosen based on minimization of the log likelihood. This process is computationally expensive, relative to response surfaces, as the hyper-parameters are optimized by a gradient-based optimizer for the dataset and have to be re-optimized if new data is added and the optimal model is still desired. However, compared to the computational cost for one CFD run, computational time is insignificant. Also, once a model is built and no more data points need to be added, an optimization step is no longer needed, further reducing computation time.

An additional benefit of Gaussian process metamodels is that they can better capture complex fluid behavior which a response surface would average out across the entire design space $^{28}$. Figure 5-1 shows one such study where a complex function including exponential, sinusoidal and step function behavior was modeled. It is important to note that to really resolve the step a large number of points were needed which corresponds to a lot of data. This may be impractical depending on the time it takes to get data points but does display the ability to capture such flow features.
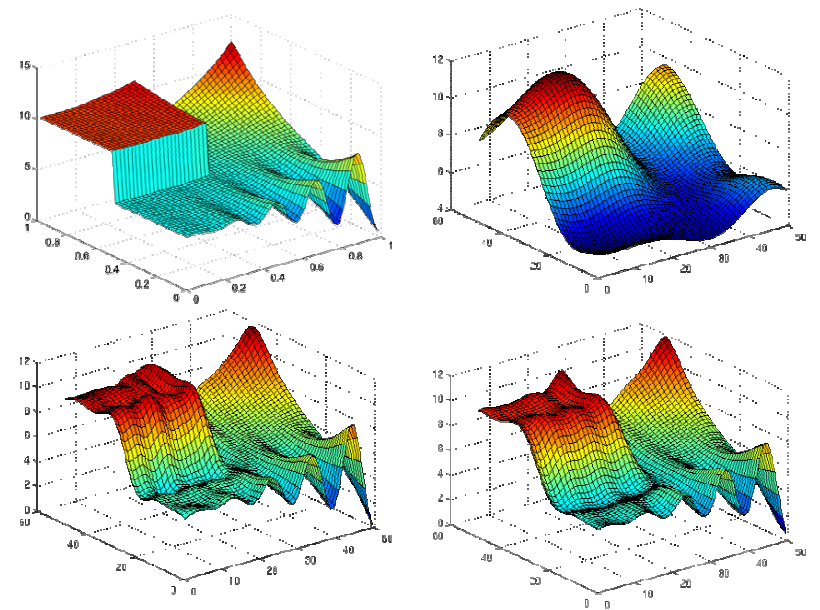

Figure 5-1: Complex Behavior modeled by Gaussian Process 
Once the hyperparameters are optimized they are then used along with the training data points to build the model. The number and effect of hyper-parameters are dependent on the covariance function chosen for the model. Covariance functions are analogous to basis functions in that they define the general shape and behavior of the Gaussian process model, but are still highly flexible. The hyperparameters are coefficients within the covariance functions that define the characteristic length scale of the function. This determines approximately how far the function can go before it has a large change in magnitude. Large length scales correspond to global models where many points affect the overall shape of the model, such as a response surface. Small length scales correspond to models that are affected by only neighboring points, such as splines. This idea is shown in Figure 1-1 reproduced from Gaussian Processes for Machine Learning ${ }^{29}$.
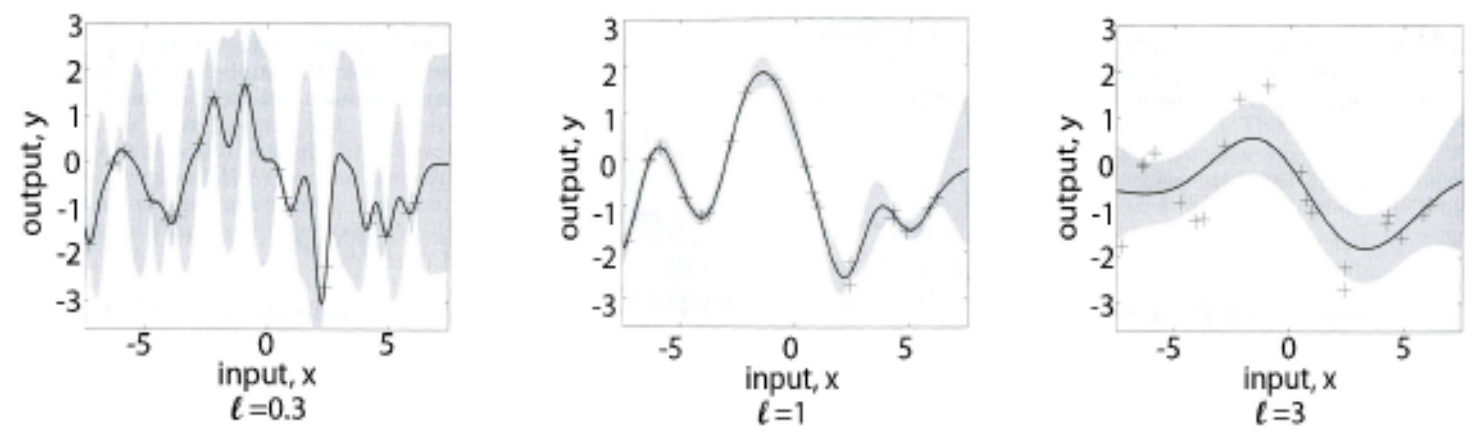

Figure 5-2: Illustration of the use of Length Scale in Gaussian Processes ${ }^{29}$

By optimizing the hyperparameters based on the log likelihood, the model that best fits the points can be created. The covariance function used for the initial work was a squared exponential covariance function, which was chosen to model the smooth behavior expected for subsonic takeoff aerodynamics.

The purpose of creating the model was so that the equations of motion could be numerically integrated over the course of a take-off run and the balanced field length 
could be calculated for a 3D CCW. Balanced field length calculations are computationally expensive by nature, since balanced field length is defined as the distance traveled to clear an obstacle at the end of the runway or come to a complete stop in the same distance given an engine failure on takeoff. With the engine failure point unknown, the equations of motion must be solved iteratively to establish when an engine out condition yields equivalent braking and takeoff distances. To allow for visual exploration of the design space, a graphical tool was created that allowed for visualization of the multidimensional Gaussian process the same way one could explore a response surface with the Matlab response surface tool. This tool can be seen in Figure 5-3 based of the 4D interim results.

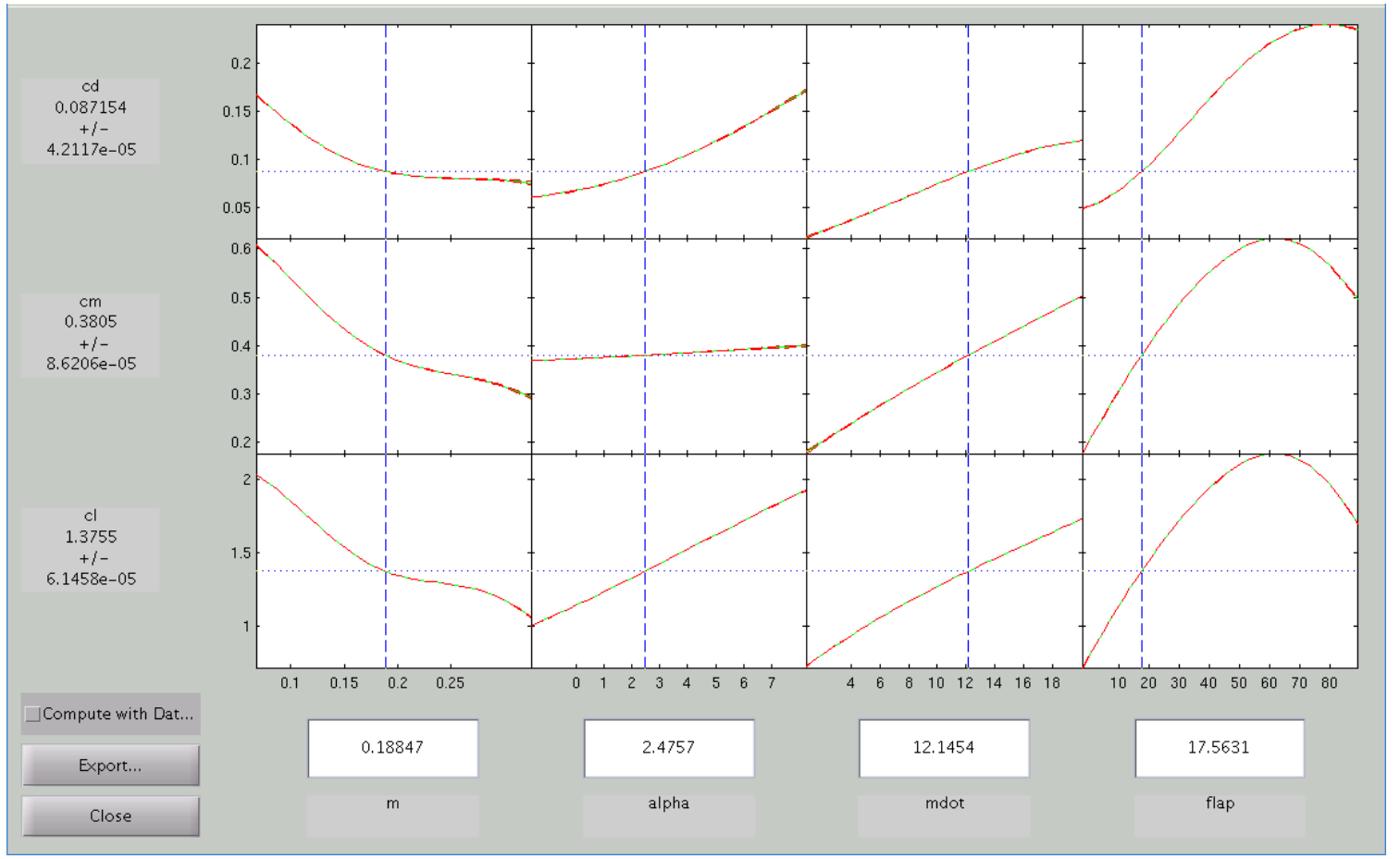

Figure 5-3: Gaussian Process Graphical Interface

The GP tool allowed the user to input the four design parameters, Mach number, angle of attack, mass flow rate, and flap deflection, and the tool would then calculate the 
values of the response data: $C_{L}, C_{D}$, and $C_{M}$ and display the values on the left. The plot windows showed how the design parameters correlated with each other. For example, from the figure it can be noted that the angle of attack and mass flow rate appear to be mostly linear as the variables increase while the Mach number and flap deflection show significant nonlinear qualities. From the plot it was also determined that the maximum lift occurred near a $60^{\circ}$ flap deflection. This was used later in performance calculations for BFL.

The ability to see individual variable effects was another beneficial advantage of modeling the multivariate data. For example, the Gaussian process was able to show the large loss of lift due to the significant separation at high angles of attack and flap deflection which prompted the previously detailed turbulence study. In this way the metamodel allowed for a focused look at a particular region of the flow in a large dataset.

\subsection{BFL Simulation}

With a metamodel constructed for the preliminary data set, it was possible to build a balanced field length code that integrated the equations of motion and used the Gaussian process model as the aerodynamic model for the aircraft. The equations of motion were coded in Matlab using the following assumptions to simplify the implementation of the model. Rotation was implemented as three seconds of additional ground roll after which the angle of attack was set to the climb angle of attack. Moments were ignored and rotation was assumed to be possible. Transition was not included and climb was assumed to follow rotation. A simplified block diagram shown in Figure 5-4 shows one loop of the BFL code and which model is used in each segment of the takeoff calculation. This resulted in a 3 degree of freedom model. 


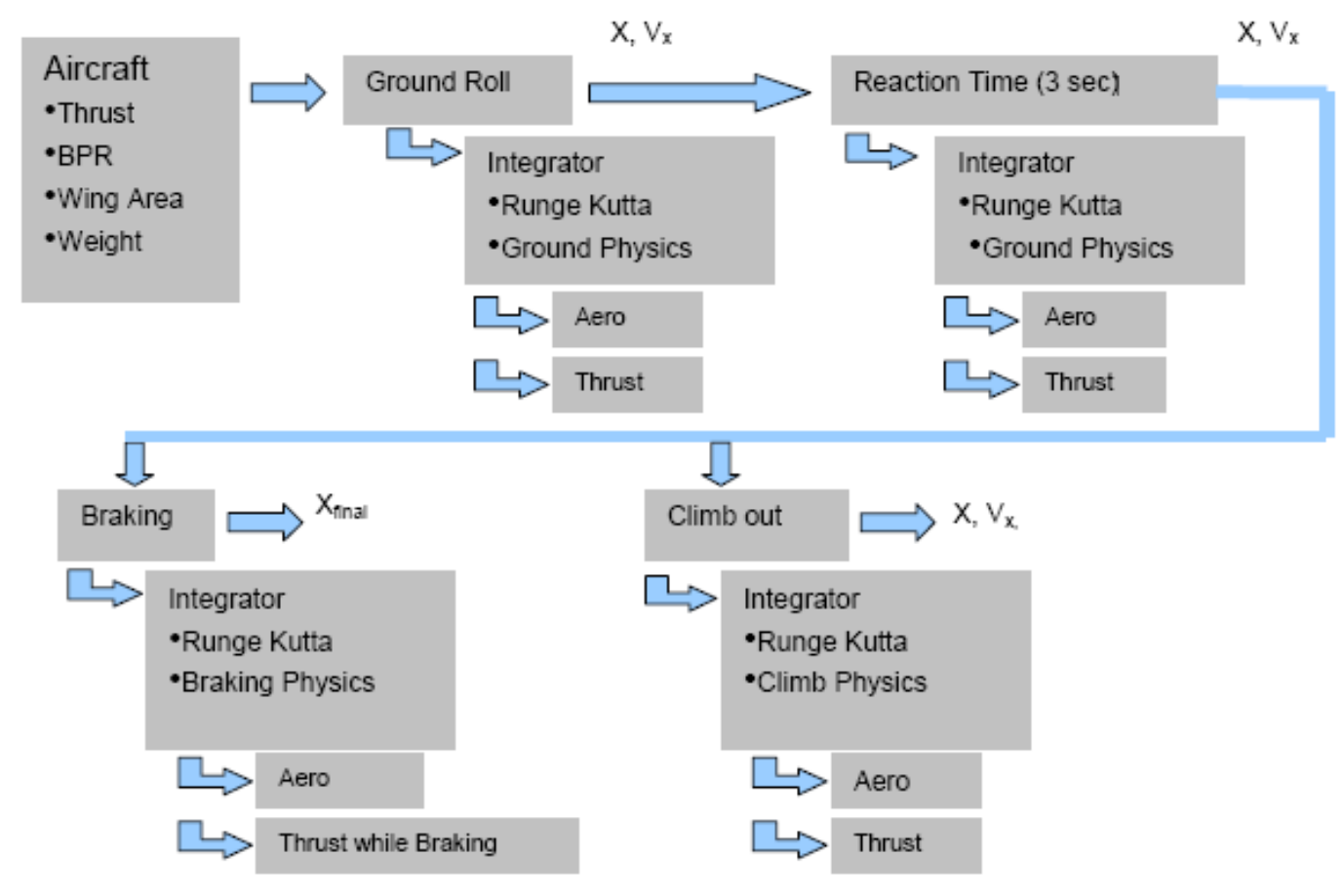

Figure 5-4: Block Diagram of Takeoff Model

There are additional assumptions that are built into the aerodynamics and thrust models. For the preliminary and interim results, the thrust model follows an assumed quadratic variation, which was determined from three known data points for two validation models. The aerodynamic model was assumed to be uncoupled from the propulsion model by the use of imbedded gas generators for the mass flow production. The drag of the wing is taken to be the total drag of the aircraft and no ground effect was taken into account. A Runge Kutta ordinary differential equation solver was used to integrate the equations of motion at different points in the ground roll. Each segment has a separate integrator so that different conditions could be implemented with the same physics model. For instance, while the rotation/reaction interval uses the same physics as the ground roll, it would be possible to switch out that physics model and integrate across a rotation as well as a velocity. The modular format also allows for implementation of 
different aerodynamic and thrust functions at different points in the takeoff. As will be examined in detail, the mass flow rate could be changed mid take off and the effect could be compared with a quadratic increase in mass flow. The architecture structure leaves room for many types of preliminary takeoff analysis all that is required is a thrust model, an aerodynamics model, and some basic aircraft parameters.

\subsubsection{Performance Validation}

In order to determine whether the results provided by the model were accurate, it was necessary to compare them to known results. Two validation cases were taken from an undergraduate study of preliminary takeoff analysis programs ${ }^{30}$. Among other things, the report aimed to compare different methods for using the equations of motion to analyze takeoff and compare those to known results. The known results which were used to validate the current model included data for the McDonnell Douglas DC-9 from NASA Langley's Flight Optimization System (FLOPS) and also flight test data ${ }^{31}$ for a Boeing 747-400. Those results were compared to two existing methods: "the simplified method proposed by Powers ${ }^{32}$ and the modified version of the method proposed by Krenkel and Salzman ${ }^{33}$."

Since the DC-9 FLOPS results included the results for BFL, it became the first validation case for the model. All of the aircraft parameters which were used it the FLOPS model were also included with reference 30 and were easily entered into the current model. These parameters included the quadratic thrust model, thrust, weight, wing area, and the assumed $\mathrm{C}_{\mathrm{L}}$ and $\mathrm{C}_{\mathrm{D}}$ for the different phases of takeoff. The detailed $\mathrm{CC}$ aerodynamic block was replaced with the simplified assumptions which were used in the FLOPS model in order to match the results. The only significant difference between the 
two models was the method for determining the rotation velocity. This validation case employed a modified rotation velocity calculation which varies from traditional methods of which FLOPS was assumed to use. For more detail about this modified rotation method, see Appendix D. With all of the input data entered into the current model, the BFL was calculated and the results are illustrated in Figure 5-5 and listed in Table 5-1. Although the different velocities tended to vary up to $12 \%$ from the FLOPS results, the overall distances were much closer with takeoff and BFL being about $4 \%$ and $7 \%$ different respectively. It is also interesting to note that the rotation velocities were relatively close with the modified rotation method predicting the occurrence at about $10 \%$ slower speeds. This variance seems reasonable when considering the assumptions that were made deriving the modified method.

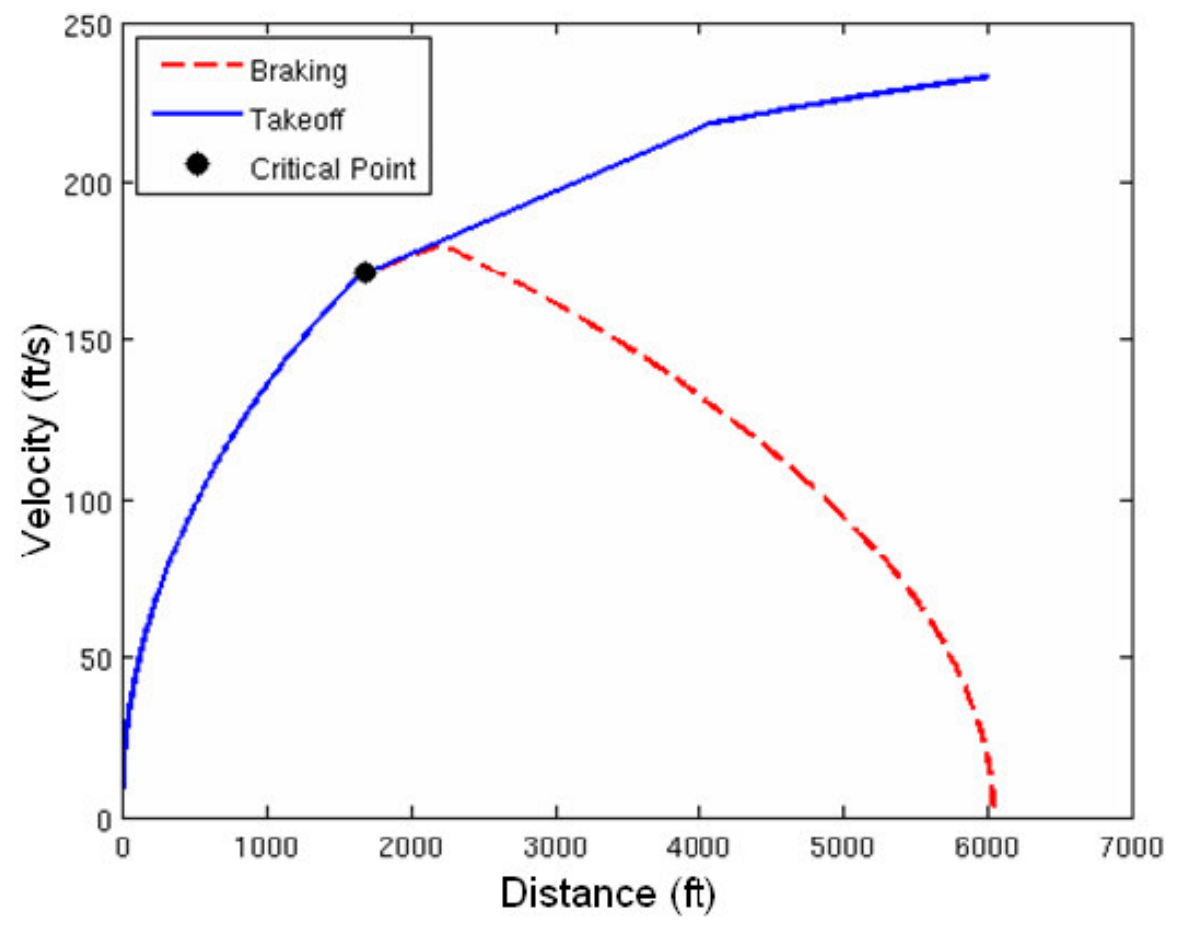

Figure 5-5: DC-9 Validation BFL 
Table 5-1: DC-9 Validation Results

\begin{tabular}{|l|c|c|c|}
\hline \multicolumn{1}{|c|}{ Parameter } & Our Model & FLOPS & \% Difference \\
\hline$V_{\mathbf{r}}(\mathbf{f t} / \mathbf{s})$ & $\mathbf{1 9 8 . 2}$ & $\mathbf{2 2 1 . 3}$ & $\mathbf{1 0 . 5 6}$ \\
\hline$V_{\text {lo }}(\mathbf{f t} / \mathbf{s})$ & $\mathbf{2 1 8 . 1 8}$ & $\mathbf{2 4 9 . 1}$ & $\mathbf{1 2 . 4 1}$ \\
\hline$V_{\text {ob }}(\mathbf{f t} / \mathbf{s})$ & $\mathbf{2 4 7 . 7 7}$ & $\mathbf{2 6 8 . 3}$ & $\mathbf{7 . 6 5}$ \\
\hline$S_{\text {to }}(\mathbf{f t})$ & $\mathbf{4 5 1 8}$ & $\mathbf{4 3 9 7}$ & $\mathbf{4 . 0 7}$ \\
\hline BFL $(\mathbf{f t})$ & $\mathbf{6 0 2 7}$ & $\mathbf{5 6 2 3}$ & $\mathbf{7 . 1 8}$ \\
\hline
\end{tabular}

The other validation case was that of a 747-400 with its flight test results ${ }^{30} 31$. Since the known results were from a flight test, was difficult to reconstruct an actual airplane to fit into a preliminary takeoff model. The same assumptions about thrust, lift, and drag which were used by reference 30 in the Powers and modified Krenkel and Salzman codes since they performed well at matching the test results within about 5\%. However, there was a discrepancy for the lift off distance with the Powers method, which produced a distance over $20 \%$ shorter than the given $7,500 \mathrm{ft}^{30}$. At any rate, the input parameters were entered into the current model. At first, there was a significant (about 18\%) difference between the takeoff distances. It was thought that it was possibly the modified rotation calculation causing some of the error so the case was run again with the traditional method for calculating rotation velocity based on the given $\mathrm{C}_{\mathrm{Lmax}}$. Those results can be in Table 3-1 and Figure 5-6.

Table 5-2: 747 Validation Results

\begin{tabular}{|l|c|c|c|}
\hline \multicolumn{1}{|c|}{ Parameter } & Our Model & Flight Test & \% Difference \\
\hline$V_{\mathbf{r}}(\mathbf{f t} / \mathbf{s})$ & $\mathbf{2 6 4}$ & $\mathbf{2 7 1}$ & $\mathbf{2 . 5 8}$ \\
\hline $\mathbf{V}_{\text {lo }}(\mathbf{f t} / \mathbf{s})$ & $\mathbf{2 7 5 . 7 8}$ & $\mathbf{2 8 3 . 4}$ & $\mathbf{2 . 7 9}$ \\
\hline $\mathbf{V}_{\text {ob }}(\mathbf{f t} / \mathbf{s})$ & $\mathbf{2 8 5 . 2}$ & $\mathbf{2 8 8 . 8}$ & $\mathbf{1 . 2 5}$ \\
\hline $\mathbf{S}_{\text {to }}(\mathbf{f t})$ & $\mathbf{8 1 2 3}$ & $\mathbf{8 6 4 5}$ & $\mathbf{6 . 0 4}$ \\
\hline
\end{tabular}




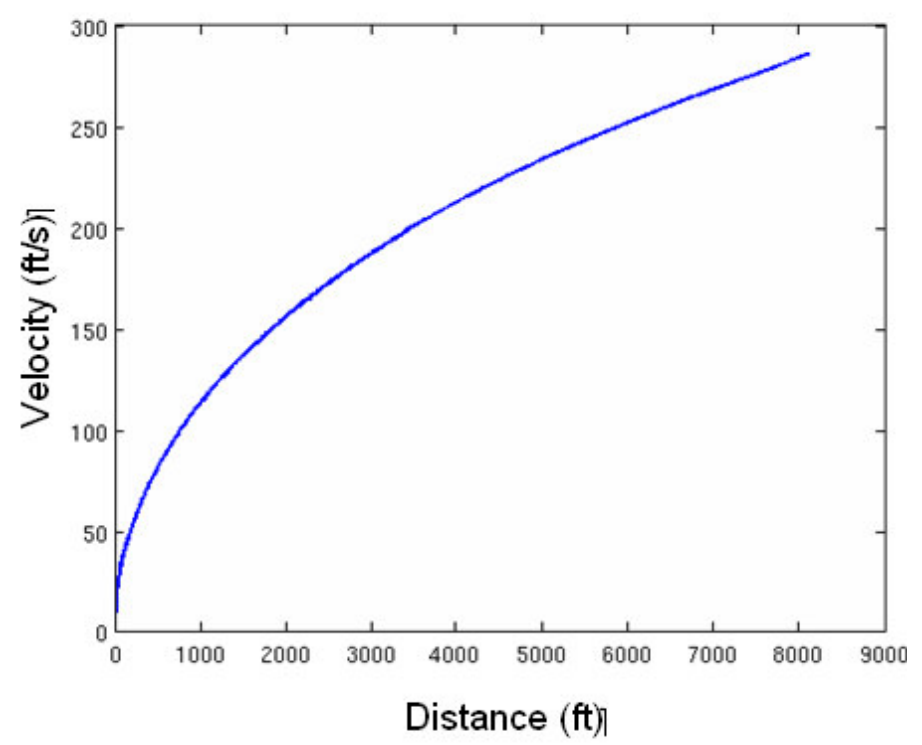

Figure 5-6: Takeoff Validation for 747

With the traditional rotation velocity calculation, the results matched very well with the flight test with error of the overall takeoff distance decreasing from $18 \%$ to $6 \%$ and the other velocities all matching within $3 \%$ or better. Although the traditional rotation calculations agreed better with the test data in this case, more research will have to be put forth to improve the idea that the rotation speed can be a function of lift over weight rather than solely $\mathrm{C}_{\mathrm{Lmax}}$. It is also worth noting that some of the error could have originated from the input data for the 747 since the Powers results ${ }^{30}$ also differed greatly from the test data. More test data or FLOPS results are needed to fully validate the model and the modified rotation velocity calculations. However, the model itself has shown that it works well at predicting takeoff performance for some traditional aircraft and next the preliminary model and results of a CCW STOL transport will be analyzed. 


\section{Preliminary Model}

This section aims to illustrate some of the major flow features present in the preliminary results. These preliminary results were obtained using the first set of grids generated using Gambit with the uniform growth technique. These preliminary results were based off of 40 cases on the thirty different flap deflections. This provided a good data set from which the performance model could be based. However, before BFL performance is discussed, a sample of the CFD results from the preliminary study will be presented. Three different kinds of results will be presented in this section. First, some 2D examples illustrating the CC effects, second some typical 3D results for the Gambit meshes on the coarser grids, and third the results of a study on the large separation region present in a number of the cases.

\subsection{Gridding with Gambit}

In order to accurately model how circulation control performed at different flap deflections, it was necessary generate several grids. As mentioned in the previous section, it was decided that 30 grids would be required to model every three degrees of flap deflection between zero and $90^{\circ}$.

\subsubsection{Gambit Limitations}

The primary software used to generate the grids for this study, Gambit, is the gridding counterpart included with Fluent. Although satisfactory grids were able to be produced using Gambit, the software does have some limitations which resulted in some gridding compromises. First, three-dimensional boundary layer cells were found to be extremely difficult to generate over the CCW. This largest part of the problem was thought to have been a result of the small notch in the geometry caused by the ejection 
slot. As a result, the grids were generated purely of tetrahedral cells which meant that the boundary layer was difficult to resolve with an efficient number of cells. In section 6.2.2 it will be discussed how $\mathrm{y}^{+}$adaption was used in an attempt to capture the boundary layer. The second major limitation to Gambit was its inability to handle geometry changes. Although a new flap deflection only meant that the flap was adjusted a few degrees, an entirely new geometry had to be generated and meshed from scratch. Additionally, this new geometry had similar but not exact reference names to its edges and surfaces which made fully automating the gridding process impossible. While these limitations were present for the preliminary and interim results, the final gridding and results was performed using grids created in Icem, a gridding software package which does not have the previously mentioned limitations, in order to check the validity of the Gambit results.

\subsubsection{Gridding Techniques}

Since the CCW was symmetric, only half of the wing needed to be modeled. The overall flow volume around the wing was a hemisphere which extended ten chord lengths forward and aft of the wing and ten chord lengths out from the root of the wing in order to capture all of the 3D effects. An example of a flow volume can be seen in Figure 6-1.
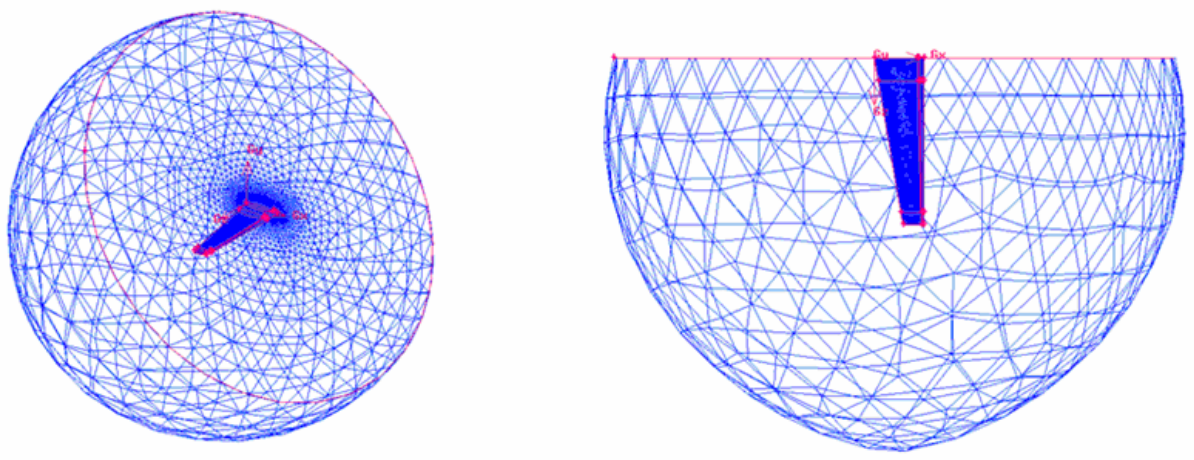

Figure 6-1: Hemispherical Flow Volume 
The basic process that was followed with Gambit for gridding involved specifying edge node distributions to provide proper refinement and cell transitions. With the edge meshes in place, the surfaces were then meshed by specifying what Gambit calls a sizing function. The sizing function was used to generate surface and volume meshes by growing the edge and then surface meshes at a specified rate. The flow volume was meshed using a meshed sizing function that was based off of all of the previously meshed surfaces as well as the symmetry plane.

\subsubsection{Gridding Automation}

Since very little difference existed in the wing geometry for $3^{\circ}$ flap increments, it was the goal to institute an automated method to generate the grids. Although Gambit allows the use of pre-written script files known as journal files to generate grids, the process was never able to be fully automated for reasons previously discussed. As a result, the process was only semi-automated. The reference points for each wing had to be defined by hand for each grid which then allowed for the remaining edge, surface, and volume grids to be completed automatically. The final grids were unstructured with about 1.2 million tetrahedral cells defining the wing surface and flow volume and an example of one of them can be seen in Figure 6-2. 


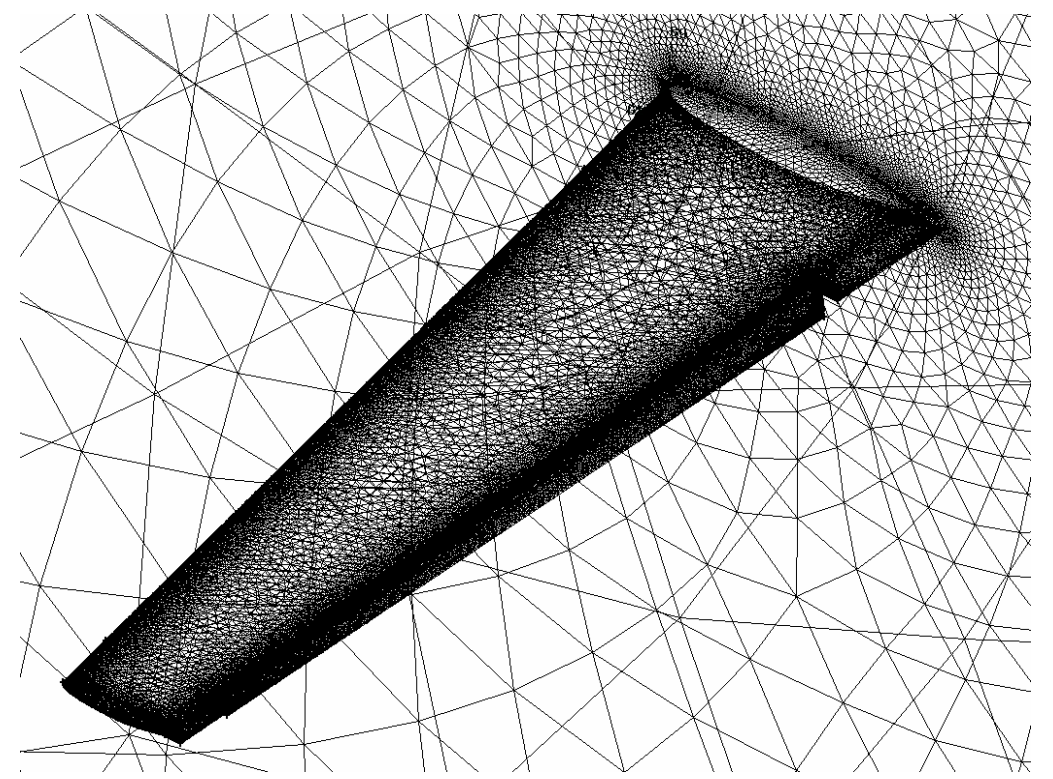

Figure 6-2: Example of a Grid Generated over the Circulation Control Wing

\subsection{Solving Methods}

With all of the required grids generated, the next step was to solve them in Fluent using the test conditions generated by the Monte Carlo of the design variables: Mach number, angle of attack, mass flow rate, and flap deflection. This section will summarize the major steps in setting up a case in Fluent as well as some solution acceleration methods which were employed.

\subsubsection{Setting up Fluent}

Setting up a Fluent case file requires a significant number of steps. First, the correct version of Fluent was initialized with the proper number of cores specified. Most of the cases for the first two models were run using the three dimensional version utilizing the two cores on the available computers. By specifying the number of cores, Fluent can then automatically partition the mesh for the different processors. After the grid has been read in and partitioned, the next major step is to specify the boundary 
conditions. For this research, there were only three different boundary conditions: pressure-far-field for the outer boundary, mass-flow-inlet for the ejection slot, and wall for the remainder of the wing. The freestream Mach number and temperature were specified directly within the pressure-far-field properties. In order to obtain results for various angles of attack without altering the grid, the direction of the freestream could be altered from the farfield boundary to produce the desired angle of attack. For the ejection slot boundary condition, the mass flow rate was entered directly in $\mathrm{kg} / \mathrm{s}$.

After the proper boundary conditions were specified, the next major step was to specify the turbulence model and multigridding which was to be used. The SpalartAllmaras model was used for this research with all of the default options provided by Fluent with the density based explicit solver. Four levels of multigridding were used with the preliminary and interim results as well as residual smoothing which allowed for a lower number of iterations to convergence. The specifics as to how those techniques work are presented in the next section. Due to the introduction of a small amount of error, multigridding was not used for the final results.

With the turbulence model set, the next step was to set the force monitors. Since the angle of attack was specified by modifying the freestream direction, the lift and drag force vectors needed to be altered so as to be perpendicular and parallel to the freestream respectively. If this step was neglected, the end results would have been entirely erroneous. Similarly as important was the final step of setting the reference conditions. If the reference area, length, and freestream dynamic pressure were not set correctly, the final lift, drag, and moment would have been severely scaled up if left with the default values. 
All of these steps as well as some other details, such as specifying the working fluid as air ideal gas and modifying the convergence criteria, were required for each Fluent case setup which would have resulted in at least 5-10 minutes of error prone tedious work for each case that was run. Since each case that was run was identical except for the four design parameters, the set up process was automated so as to eliminate the repetitious work of setting up the individual cases. The details of this automation will be presented in a section 6.2.4.

\subsection{2 $\quad \mathrm{Y}^{+}$Adaption}

The wall function approach was utilized throughout this research and in addition to this, the preliminary and interim results both utilized $\mathrm{y}^{+}$adaption. By using both techniques, the total number of cells in the grid could be kept to a minimum and thus reduce computational time. The adaption allowed for the first cell off the surface of the wing in the original grid to be even larger than the range previously specified. The technique involved reaching a steady state solution with the coarse grid with $\mathrm{y}^{+}$values in the several thousands to start and then adapt the grid within Fluent and reconverge. It was found that with four successive adaptions, the maximum $\mathrm{y}^{+}$of the first cell off the surface of the wing could be lowered to just above 600 with many regions even lower. An example of an adapted grid can be seen in Figure 6-3. 


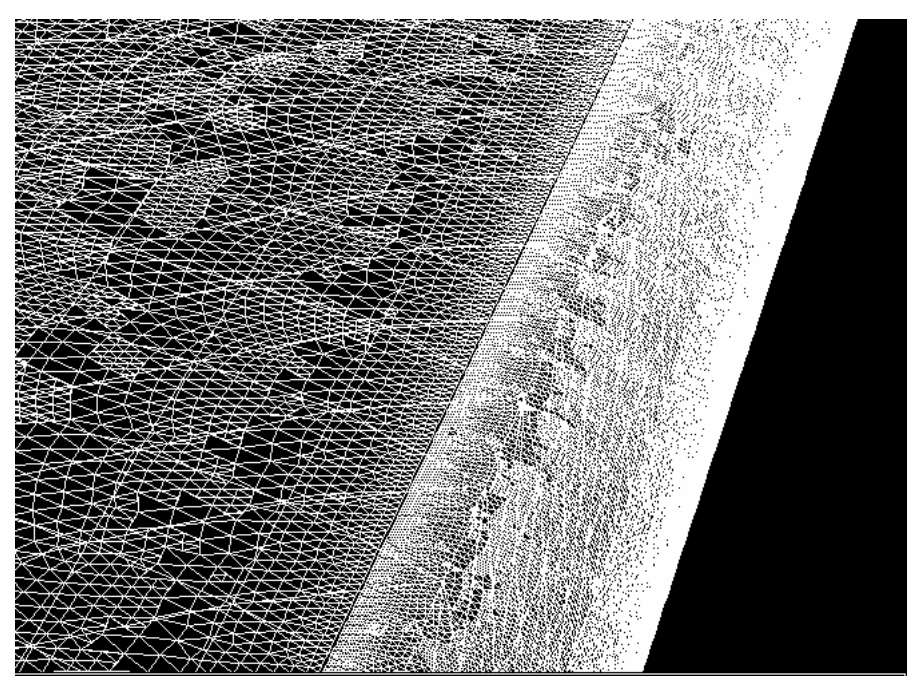

Figure 6-3: $\mathrm{Y}^{+}$Adaption on the Wing Surface and CC Flap

The tetrahedrals on a surface can be seen split in order to obtain more boundary layer refinement. Although a $\mathrm{y}^{+}$of 600 was not within the range which is considered to completely resolve the boundary layer with wall functions, computational limitations restricted any further adaptions for the preliminary grids. Each adaption added several hundred thousand cells to the grid which greatly increased the amount of time per iteration and required more memory. Four adaptions were all that the available computers could handle before running out of memory and therefore the $\mathrm{y}^{+}$of near 600 was the best that could be achieved for the preliminary and interim results. In many cases, only two or three adaptions could take place before the computers failed which resulted in $\mathrm{y}^{+}$values in greater than 2000 . However, the goal of this study was to provide a performance tool for calculating takeoff distances which only relied on the integrated values of $C_{L}$ and $C_{D}$. Even though all of different flow phenomenon near the surface was not captured, the coarse grids provided a computationally efficient approach to providing the desired performance tool. In later sections, these preliminary results will be 
compared to more refined results to add more accuracy to the performance tool and to improve upon the ability to capture the boundary layer.

\subsubsection{Multigridding}

A technique used to decrease convergence time which was investigated was the use of multigridding. Multigridding is a process of using successively coarser grids in order to eliminate low frequency error from the system. This ability is built into Fluent and, when enabled, allows the user to generate a specified number of coarser grids and control over many other properties related to the elimination of error from the system. Although sweeping through a series of grids took additional time for each iteration, it was found that the number of iterations required to reach convergence was an order of magnitude lower when multigridding was used when compared to a single grid. One of the drawbacks known to exist with multigridding is the loss of non-linear flow effects due to the interpolation between grids. To determine whether the results for circulation control wing were being altered by the multigridding, a case was solved with and without the use of multigridding and the resulting $\mathrm{C}_{\mathrm{L}}$ was found to be $1.6 \%$ different. These results are summarized in Table 6-1. These results clearly show the advantages provided by $\mathrm{y}^{+}$adaption as well as multigridding. For these cases, multigridding was initiated at different times in order to find out how best to reduce computation time. Clearly it was shown that starting the case with multigridding enabled provided the least number of iterations and the shortest run time. In addition, it can be seen here that four adaptions lowered the $\mathrm{y}^{+}$value from the $8000-9000$ range down to 638 . 
Table 6-1: Adaption and Multigridding Examination

\begin{tabular}{|c|c|c|c|c|c|c|c|}
\hline Method & $\begin{array}{l}\text { Iterations to } \\
\text { Convergence }\end{array}$ & $\begin{array}{l}\text { Run Time } \\
\text { (hrs) }\end{array}$ & Adaption(\#) & $\mathrm{Y}^{+}$ & $\mathrm{C}_{\mathrm{L}}$ & $\mathrm{C}_{\mathrm{D}}$ & $\mathrm{C}_{\mathrm{M}}$ \\
\hline $\begin{array}{l}\text { SA with } \\
\text { multigrid at } \\
2500\end{array}$ & 3000 & 15.88 & No & 8202 & 2.6234 & 0.4732 & 2.0722 \\
\hline $\begin{array}{l}\text { SA with } \\
\text { multigrid } \\
\text { from start }\end{array}$ & 650 & 12.4 & Yes (3) & 638 & 2.5830 & 0.4901 & 2.0450 \\
\hline $\begin{array}{l}\text { SA with } \\
\text { multigrid } \\
\text { from start }\end{array}$ & 500 & 3.75 & No & 8202 & 2.5980 & 0.4700 & 2.0534 \\
\hline $\begin{array}{l}\text { Laminar to } \\
\text { SA }\end{array}$ & 2000 & 4 & No & 9000 & 2.5550 & 0.4472 & 2.0187 \\
\hline
\end{tabular}

Although a significant difference was not found in the final results of $C_{L} C_{D}$ and $C_{M}$ for these test cases, this study continued to execute $\mathrm{y}^{+}$adaption in order to model the turbulent flowfield as accurately as possible.

Finally, the proper time to implement multigridding in order to minimize convergence time was investigated. It was thought that a solution might be obtained quicker if a laminar case was first converged and then the turbulence model was to be turned on. However, this was found not to be the case. The minimum convergence time was found to occur with multigridding and the Spalart-Allmaras turbulence model enabled throughout the case. It was found that before adaption, convergence occurred in less than 4 hours with 500 iterations. Once adaption was enabled, the time to iterate significantly increased which resulted in an additional 8.65 hours to iterate an additional 150 times to reach convergence. These results are also summarized in Table 6-1.

Although multigridding was shown to drastically reduce the time to convergence for these preliminary results, the amount of error which it introduced into the solutions was significant enough to warrant the disuse of multigridding for the final solutions which will be presented in section 8 for the final model. However, since the preliminary 
model was meant to be more as a learning experience, the error introduced from multigridding was acceptable.

\subsubsection{Fluent Automation Scripting}

One of the difficulties in solving several different test cases within Fluent was that setting up each individual case by hand was tedious and time consuming. In order to speed up the process of setting up and running many similar cases in Fluent, a Bash automation script was created. This script was designed to either function independently or coupled with a mesh transfer/generator script named Mesh-Sender. In its independent form, the Fluent-Setup script simply queried the user for the four design variables for the particular case, namely the Mach number, angle of attack, mass flow rate of the ejection slot, and the dual radius flap deflection. Later, it was modified to include two other variables: jet slot temperature and height off the ground. Since those were the only six parameters that were changing between runs, the Fluent-Setup script took those values, copied them into a template file and created a Fluent journal file. The script would finish by initiating Fluent with the newly generated journal file which completely set up, initialized, ran, and saved the particular case. The following sections aim to explain how that was accomplished and how the two complementary scripts work.

\subsubsection{Mesh Sender Script}

The Fluent-Setup script was designed to work with the Mesh-Sender script for use on remote computers. Before a Fluent case could be run, a mesh needed to be available for the script to call. The job of the Mesh-Sender script was query the user as to what grid was required, determine if that grid was available, and then send that grid along with 
required template and setup files to a remote computer. Once all the files were at the remote computer, the Fluent-Setup script was then executed.

The original purpose of the Mesh-Sender script was to simply move files from a source computer to the remote computer without having to type in the copy commands each time. That script was then expanded significantly to include many new features. These included several queries as to what grid was required: what would be the required flap deflection, whether or not there would be blowing, whether or not the ground will be included and if so, the altitude of the wing over the ground. The script searched for the appropriate grid and if it was found, it would send it along to the remote computer along with the required template file and some setup information. If the appropriate grid was not found, it would do one of two things. In the case of a flap deflection not found, the script prompted the user to specify a flap deflection between zero and 90 degrees at multiples of three. In the case where a ground height and/or a specific angle of attack were not available, the Mesh-Sender script actually called Gambit and created a new mesh with the specified ground height and angle of attack. Two-dimensional versions of both scripts were created and performed the same functions except that there was no restriction on flap deflection. The flap could be specified at any angle and not just multiples of three. For more detailed information about the large blocks of code in the Mesh-Sender script, see Appendix A.

\subsubsection{Fluent-Setup-Run Script}

The purpose of the Fluent-Setup-Run script was to completely set up, initialize, run, and write the converged case to a file. For the circulation control data that was being collected, the same turbulence model and boundary conditions were being used for each 
case with only minor changes in angle of attack, mass flow rate from the ejection slot, Mach number, and flap deflection. This script set up all the details required by Fluent and also copied in the required changes required by the particular case. The final case was given a name based on the four design inputs in order to ease the differentiation of the cases and speed the databasing process. A large percentage of the Fluent-Setup script was dedicated towards naming the case in a consistent manner. Each case name was the same number of digits long with truncated values of the test parameters built into the name. For example, a case with a Mach number of 0.1543 , angle of attack of 2.456 , mass flow rate of 12.123 and a flap deflection of 60 degrees would be M15A24M121F60.cas.

As was discussed in the section on design of experiments, the blowing for the CCW was specified by a mass flow rate instead of the non-dimensoinalized $\mathrm{C}_{\mu}$. However, the Fluent-Setup script allowed for the mass flow to be specified by either method, using the regression fit to predetermine $\mathrm{C}_{\mu}$ without knowing the converged jet velocity.

The majority of the work required to set up a Fluent case was not performed by the Fluent-Setup script but by the journal template file. All of the commands that Fluent was to execute were contained in the template file along with dummy variables which were replaced with the input parameters for the specific case. This technique easily allowed for a new template file with different conditions to be created and used with the script. For example, the boundary conditions had to be modified in order to run cases with no blowing on the CC flap. An additional template file was created based off the standard version with modifications to take care of the new boundary conditions. In the end, there were four main template files that were used to set up and run the cases 
determined by two parameters: whether or not there was blowing and whether or not there was ground effect.

An additional feature to the Fluent-Setup script was the option to run Fluent with or without the graphical interface. Since the setup script itself could be run from the command line, adding the feature to run Fluent entirely from the command line eliminated the need to pass unnecessary graphics between computers. In addition, if cases were to be run on a remote cluster, it is often a requirement that the case be run entirely from the command line since sending graphical information will slow down the computation. For more details about the Fluent-Setup script, see Appendix B. Also, the standard template file, which included the case with blowing and no ground effect, is included in Appendix C.

\subsection{D Results}

The first case which will be presented occurred at a Mach number of 0.15 , an angle of attack of $4^{\circ}$, a mass flow rate of $9.4 \mathrm{~kg} / \mathrm{s}$, and a dual radius flap deflection of $66^{\circ}$. Figure 6-4 shows this case with the streamlines colored by Mach number. The basic principles and performance of CC can clearly be seen in this figure. The high speed mass flow can clearly be seen ejected over the dual radius flap and continue down into the flowfield. Although the flap is only at $66^{\circ}$, the curvature of the two radii clearly deflect the flow at what appears to be nearly $90^{\circ}$. As a result of this, the forward stagnation point can clearly be seen shifted significantly down the lower surface of wing while the rear stagnation point lies at the tip of the flap. The curvature of the pathlines in the immediate flowfield indicates a significant increase in camber and therefore a reasonably high lift coefficient. 


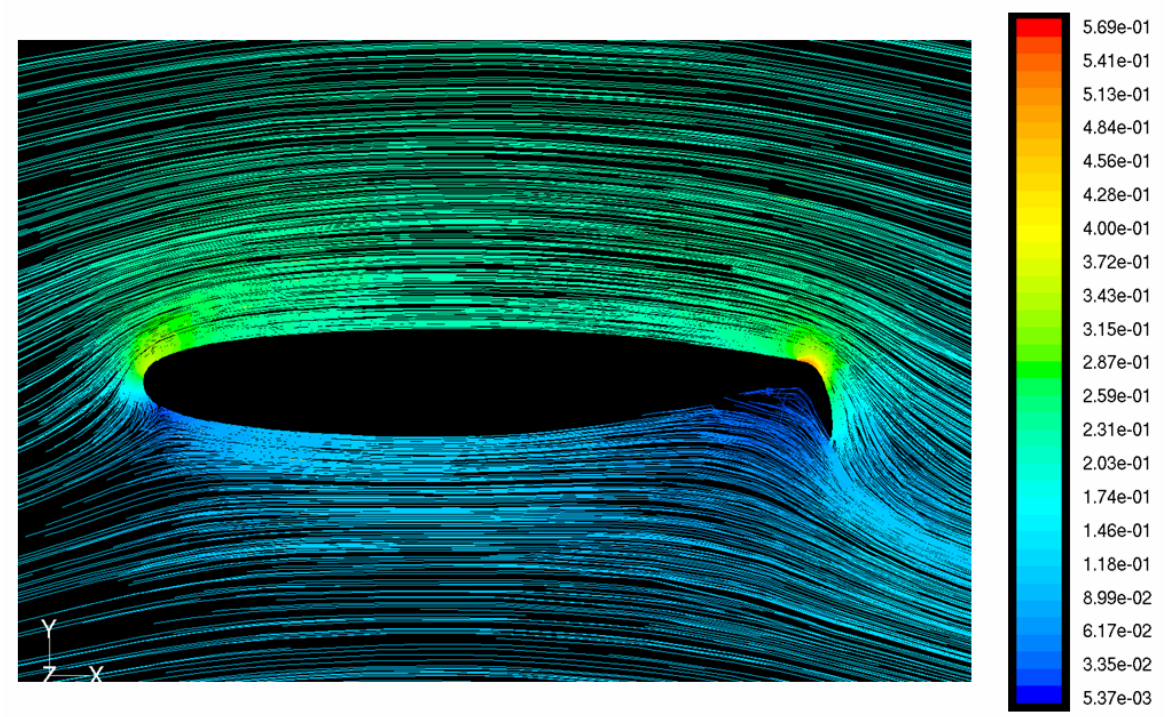

Figure 6-4: Typical CC Streamlines in 2-D, colored by Mach number

\subsection{D Typical Flowfield Results}

Although the major $\mathrm{CC}$ characteristics are easily shown in $2 \mathrm{D}$, three dimensional results were required in order to accurately resolve the lift and drag of $\mathrm{CCW}$. One of the important 3D effects can be seen in Figure 6-5.

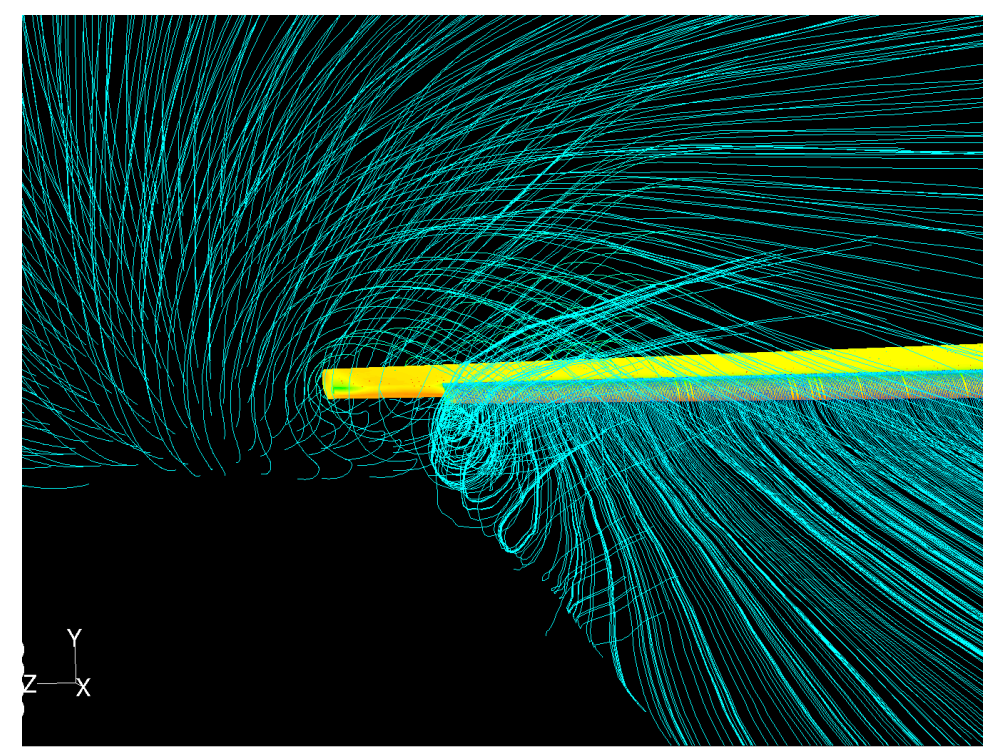

Figure 6-5: Two Tip Vortices Present in 3D Flow 
These results were for a Mach number of 0.081 , angle of attack of $5.7863^{\circ}$, mass flow rate of $5.1928 \mathrm{~kg} / \mathrm{s}$, and a flap deflection of $30^{\circ}$. Two distinct vortices are clearly present at the tip of the wing and the end of the $\mathrm{CC}$ flap. By capturing these vortices the solution was able to predict results for the induced drag and the pressure distribution and subsequently produce accurate values of $\mathrm{C}_{\mathrm{L}}$ and $\mathrm{C}_{\mathrm{D}}$.

\subsection{High Separation Results}

Although a majority of the preliminary results were similar to those of section 6.4 , this was not the case for high mass flows at high angles of attack and high flap deflections. For these cases, significant recirculation regions were found trailing the dual radius flap. An example of this can be seen in Figure 6-6 with the case defined as follows: Mach number of 0.15 , angle of attack of 7.9 degrees, mass flow rate of 19.9 $\mathrm{kg} / \mathrm{s}$, and a flap deflection of $90^{\circ}$. The separation can be seen more clearly in the $2 \mathrm{D}$ case shown in Figure 6-7. The only differences in the 2D case are that the flap deflection was reduced by $2^{\circ}$ to $88^{\circ}$ and the mass flow was set at $0.8 \mathrm{~kg} / \mathrm{s}$ to most closely match the jet velocity of the $19.9 \mathrm{~kg} / \mathrm{s}$ mass flow in three dimensions. This case illustrates the near maximum of three of the input parameters: angle of attack, mass flow rate, and flap deflection. The result of this extreme case can be seen by examining the location of the forward stagnation point. The freestream flow is being forced to turn all the way around the leading edge of the wing and then attempt to turn well over $90^{\circ}$ at the flap. 


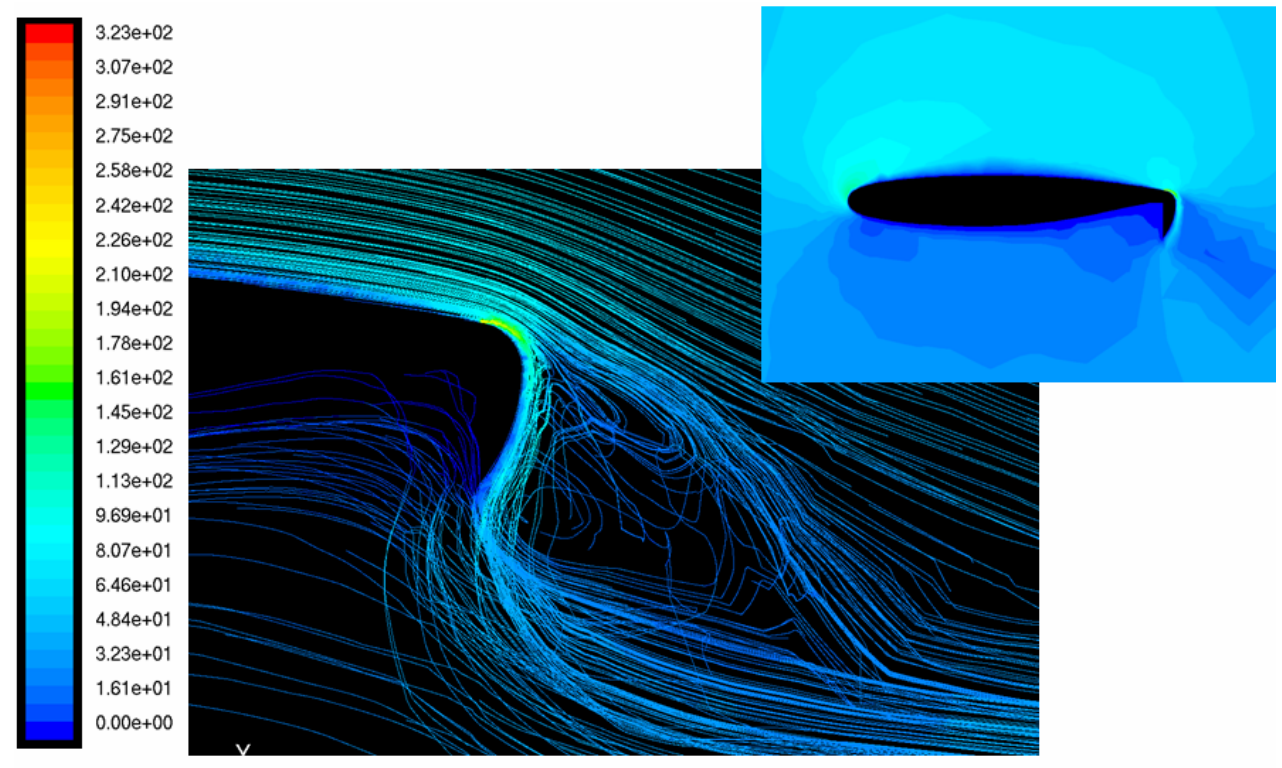

Figure 6-6: 3D Streamlines and Contours Illustrating Severe Separation (velocity streamlines, $\mathbf{m} / \mathbf{s}$ )

With the exception of the extremely high speed flow coming out of the slot, almost none of the flow turns completely around the flap. As a result, the large recirculation region appears directly aft of the airfoil and wing.

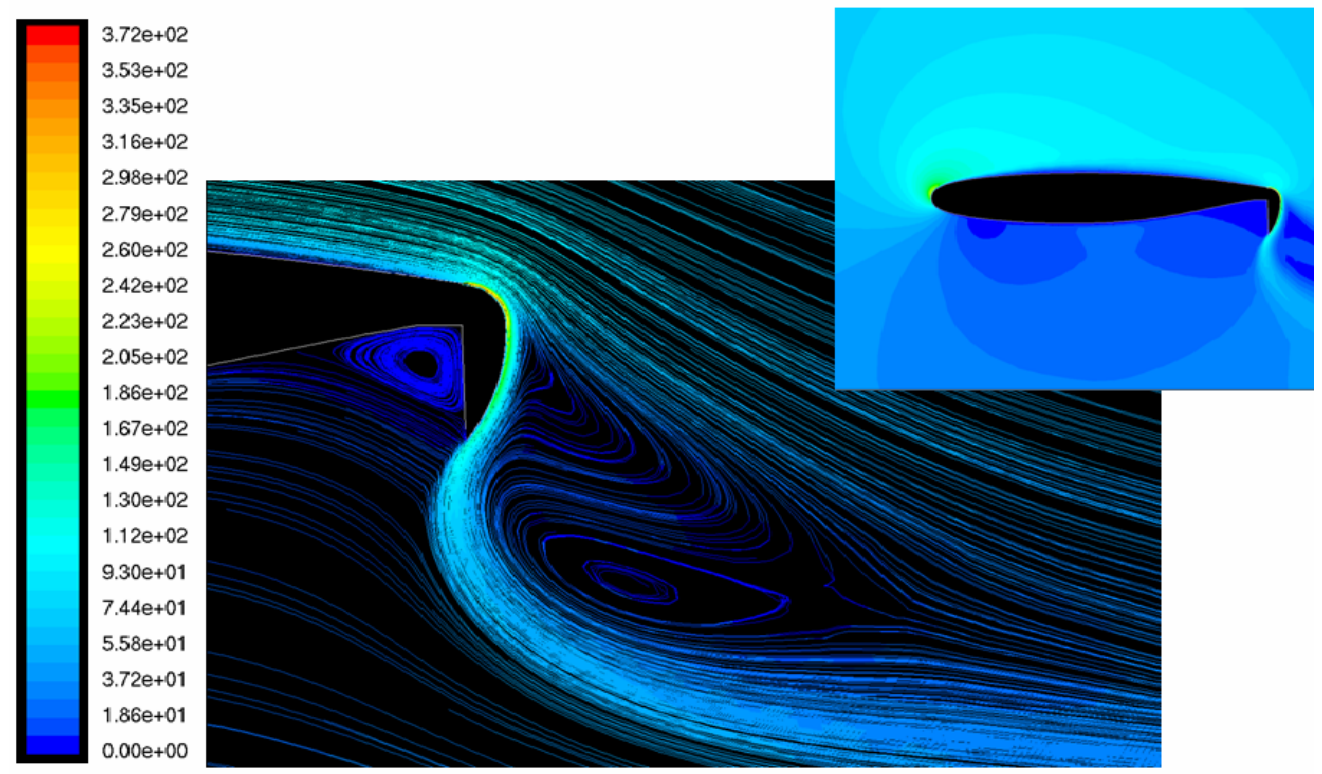

Figure 6-7: 2D Representation of Figure 6-6 (velocity streamlines, m/s) 
This loss of entrainment ended up significantly reducing the lift generated by the CC. On a slightly more positive note, the net drag ended up decreasing at the high flap deflections. This was determined to be a result of the decreased induced drag which decreased more than the added parasite drag from the recirculation region. When these results were presented ${ }^{26}$ in Reno, a question arose as to the validity of the extreme separation and whether or not any experimental data validated the results. In absence any available highly separated $\mathrm{CC}$ experimental work, the author chose to perform an additional validation for Spalart-Allmaras with the wall function approach and compare its performance to the two-equation models k- $\varepsilon$ and k- $\omega$ SST for highly separated CC flows which was presented in section 3.2.

\subsection{Preliminary Performance of CCW STOL Aircraft}

With the BFL code verified, a flap deflection and mass flow rate study was completed to determine optimal settings for BFL reduction. The result was that balanced fields lengths as low as 2400 feet could be achieved but those were obtained with diminishing returns since mass flow rates of $20 \%$ less could obtain a BFL of 2500 feet. This analysis was based on a STOL transport designed for the 2007 AIAA undergraduate design competition seen in Figure 6-8.

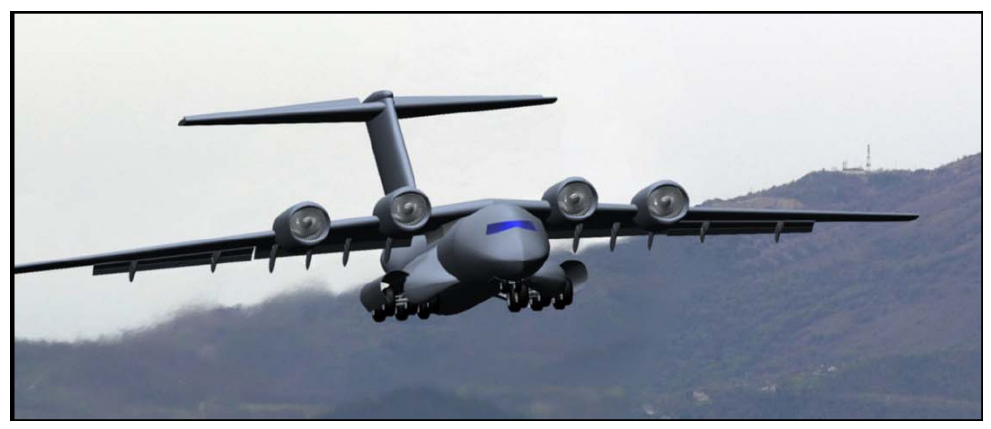

Figure 6-8: STOL Transport used in CCW BFL Model $^{26}$ 
Having recently finished this design competition, using this aircraft provided a design for which numbers for drag, engine sizing, and airfoil performance were all known. Perhaps most importantly, it provided a thrust, weight and wing area that were designed for a 2500 foot BFL, which helped with debugging and is also the range of current industry STOL transport work. The aircraft had a thrust of 130,000 lbs, a wing area of $2,200 \mathrm{ft}^{2}$, and a weight of $230,000 \mathrm{lbs}$ which gave it a thrust to weight ratio of 0.565. The thrust was increased above the original design up to $130,000 \mathrm{lbf}$ to provide a wider range of feasible takeoff conditions. The results of the mass flow and flap deflection study are shown in Figure 6-9.

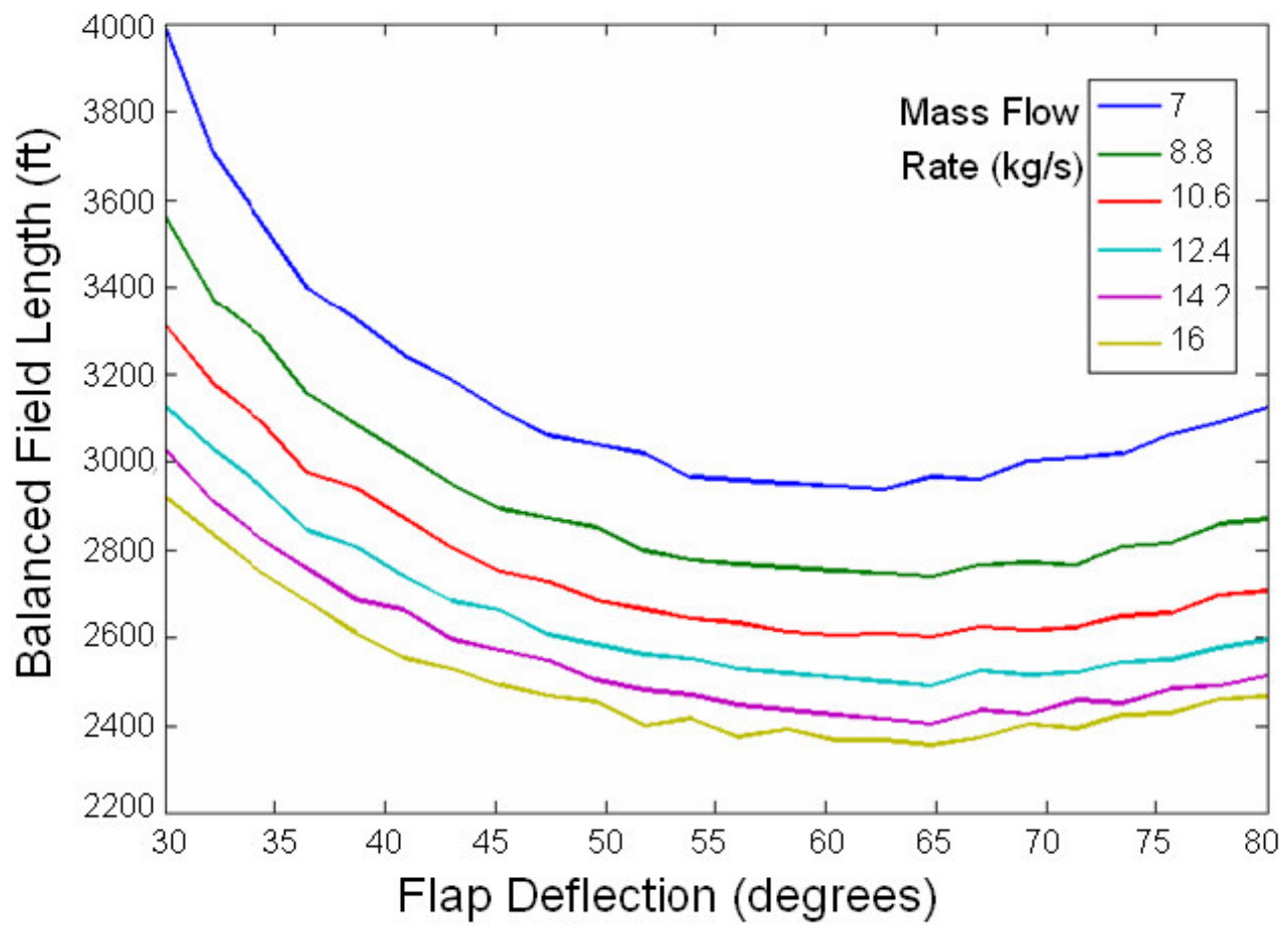

Figure 6-9: BFLs for STOL Concept with varying Mass Flow and Flap Deflection

As the flap deflection was increased there becomes a point where a minimum BFL was reached for any particular mass flow rate. This was due to the loss of 
supercirculation caused by the separated flow off the CC flap. One might expect that there would be a sharp change at this point due to increased drag caused by the recirculation region, but after looking at the metamodel and data points, it was noticed that the drag actually goes down in the regions that experience this separated flow. The reason the total drag decreased was the loss of lift caused a reduction of induced drag. This decrease in drag reduced the BFL while the loss in lift acted in the opposite direction. The net overall effect was that the shape of the curve changed its radius of curvature as compared to the small flap deflections and the BFL distances increased at a lower rate than they decreased. The mass flow rate term, unlike the flap, always provides an additional benefit to BFL, but the amount by which it improves is clearly diminishing. For a $22 \%$ decrease in mass flow, you lose only $4 \%$ of your balanced field length (2400 feet to 2500 feet). A possible explanation of this is that with additional flow the shear layer has a higher gradient across it and little additional energy is transferred into the adjoining flow. And finally, it is interesting to note that for high mass flow rates, the effect of flap deflection decreases.

The second major performance analysis was performed to determine the effects of varying the mass flow rate and flap deflection at different points in the takeoff. Specifically, it was the goal to determine the benefits of delaying the mass flow or flap deflection in order to reduce induced drag and therefore aid in the acceleration in the ground roll. Both flap deflections and mass flow were varied separately throughout the ground roll and compared to cases where they were held constant. The first case which was explored was the delay in mass flow rate. The flap was held constant throughout these cases at the optimum of $64^{\circ}$. Two different mass flow variations were chosen to 
investigate: 1) switch from essentially no mass flow to 10 or $20 \mathrm{~kg} / \mathrm{s}$ at a designated velocity in the ground roll and 2) vary the mass flow quadratically from zero to 10 or 20 $\mathrm{kg} / \mathrm{s}$. The first case was designated as the "step" input of mass flow while the second as the quadratic variation. Four cases of step inputs were examined which involved stepping at $100 \mathrm{or} 180 \mathrm{ft} / \mathrm{s}$ and for each of those, stepping to 10 or $20 \mathrm{~kg} / \mathrm{s}$. Two cases of quadratic variations were examined involving increasing the mass flow from zero to a maximum of 10 or $20 \mathrm{~kg} / \mathrm{s}$ at $180 \mathrm{ft} / \mathrm{s}$. An illustration of the step and quadratic variations to $20 \mathrm{~kg} / \mathrm{s}$ is shown in Figure 6-10.

Figure 6-10: L/D of a Takeoff with Variations in Mass Flow Rate

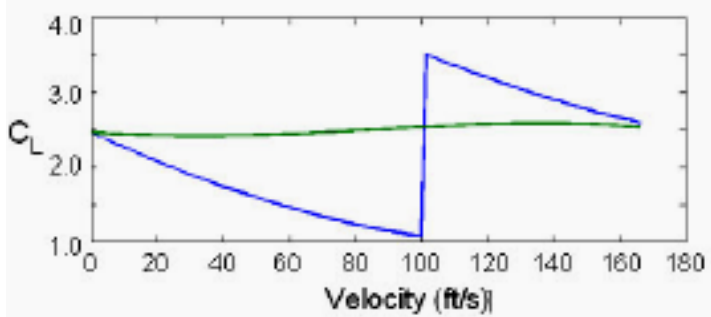

(a)

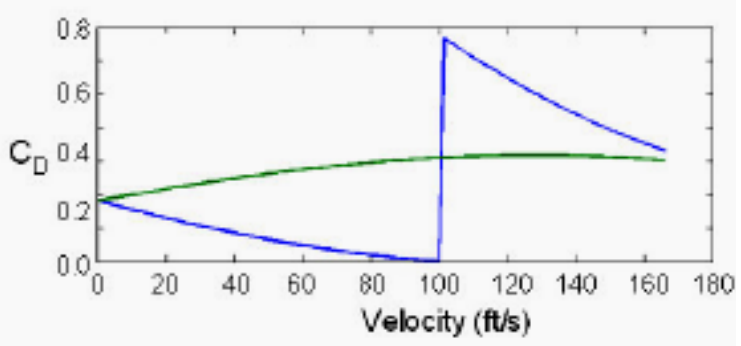

(b)

Figure 6-11: Variations in a) $C_{L}$ and b) $C_{D}$ over Takeoff with Varying Mass Flow

The step input of mass flow at $100 \mathrm{ft} / \mathrm{s}$ is clearly visible with the drastic decrease in $\mathrm{L} / \mathrm{D}$ at that point. It can be seen that although the $\mathrm{C}_{\mathrm{L}}$ at that point jumped roughly from 1.5 to 3.5 , the $C_{D}$ jumped from 0.2 to 0.8 which in turn decreased the $L / D$ value. This clearly shows one of the major difficulties of a powered lift aircraft which is that producing a lot of lift also produces a lot of induced drag. This is the reason why most STOL transports have thrust to weight ratios near or above 0.5 . The quadratic variation was also of interest to examine. The $\mathrm{C}_{\mathrm{L}}$ actually remained almost constant at a value of about 2.5 throughout the entire takeoff. The reason it remained constant was due to the 
effect of Mach number on CC which had been discovered previously with the metamodel. As the Mach number increased, the ability of the mass flow to entrain the freestream flow decreased and therefore the lift decreased. This decrease in performance for the $\mathrm{CC}$ was counteracted by the quadratic increase of mass flow. This may prove to be a useful comparison to traditional flapped wing takeoff since they generally are engaged for the entire ground roll providing a constant $\mathrm{C}_{\mathrm{L}}$. The results from the different mass flow trials can be seen in Table 6-2.

Table 6-2: Results for Mass Flow Variations

\begin{tabular}{|c|c|c|c|c|c|c|c|c|}
\hline & \multicolumn{7}{|c|}{ Speed at which Blowing was Turned on (ft/s) } \\
\hline & & \multicolumn{2}{|r|}{100} & \multicolumn{2}{|r|}{180} & \multicolumn{2}{|c|}{ Quadratic Increase to 180} & \multirow{2}{*}{$\begin{array}{l}\text { Whole Time } \\
\text { actual }\end{array}$} \\
\hline & & actual & $\%$ decrease & actual & $\%$ decrease & actual & $\%$ decrease & \\
\hline \multirow{2}{*}{$\begin{array}{c}10 \mathrm{~kg} / \mathrm{s} \\
\text { Mass Flow }\end{array}$} & Takeoff Distance (ft) & 2512 & 0.95 & 2479 & 2.25 & 2515 & 0.83 & 2536 \\
\hline & BFL (ft) & 2776 & 1.14 & 2692 & 4.13 & 2775 & 1.18 & 2808 \\
\hline $20 \mathrm{~kg} / \mathrm{s}$ & Takeoff Distance (ft) & 2191 & 0.63 & 2068 & 6.21 & 2157 & 2.18 & 2205 \\
\hline Mass Flow & BFL (ft) & 2395 & 1.68 & 2225 & 8.66 & 2357 & 3.24 & 2436 \\
\hline
\end{tabular}

From examining the results, it was clear that the largest decrease in BFL and takeoff distance occurred with the step variation of mass flow at the later speed. The maximum change occurred in the BFL distance with the $20 \mathrm{~kg} / \mathrm{s}$ mass flow step at $180 \mathrm{ft} / \mathrm{s}$ which decreased $8.66 \%$ from the BFL with the mass flow blowing the entire time. The only issue which would need to be resolved in order to perform such a takeoff would be the reliability of the mass flow source. Also, it is important to note that all of the results used in this model occurred at steady state and take no account of any transient behavior of turning on or diverting the mass flow source.

The next performance analysis involved keeping the mass flow steady and varying the deflection of the dual radius flap throughout the takeoff. A similar set of cases were set up as the mass flow variations with the flap having a step variation at certain speeds and also varying the flap deflection quadratically as the mass flow before. 
These tests were performed with the same two mass flow rates, 10 and $20 \mathrm{~kg} / \mathrm{s}$, and the step and quadratic variations behaved in a similar way as before only with the flap deflection instead of the mass flow. The results for a couple of the $20 \mathrm{~kg} / \mathrm{s}$ trials are shown in Figure 6-12.

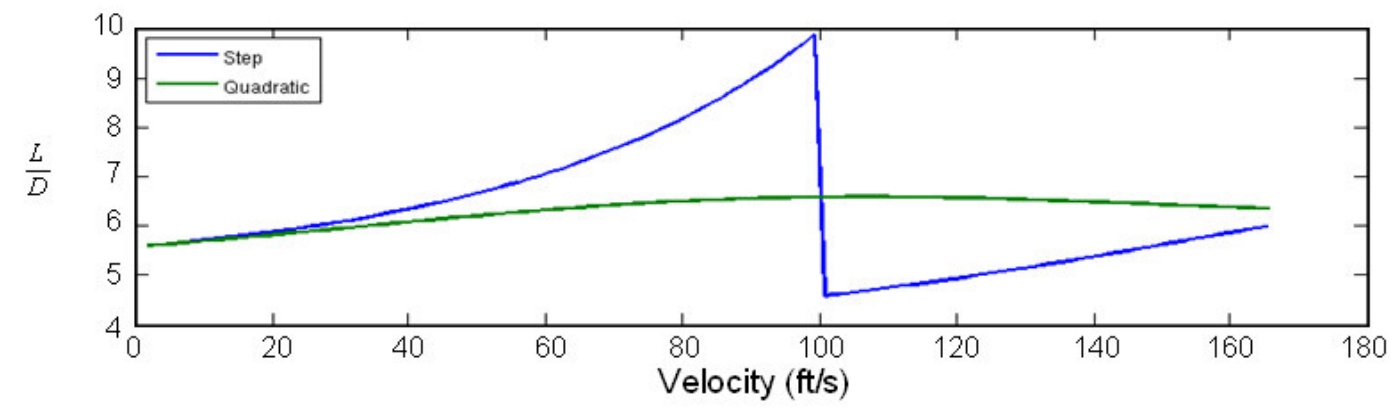

Figure 6-12: L/D throughout Takeoff with Flap Variations

The results were surprisingly similar to the variations in mass flow with only a few exceptions. For both the step and quadratic variations shown, the ratio of lift to drag began much lower than before and the step function increased at a faster rate while the quadratic variation remained almost perfectly steady at its initial value. The behavior of lift and drag individually can also be seen in Figure 6-13.

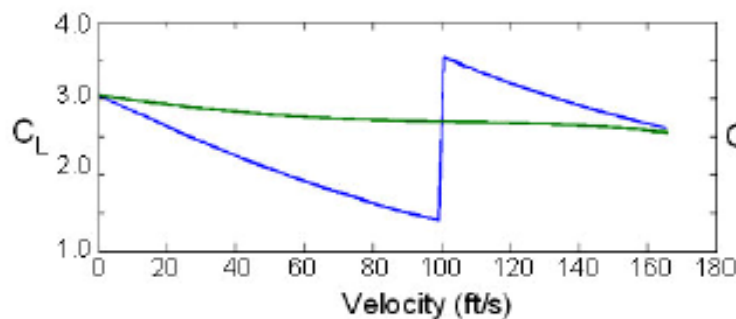

(a)

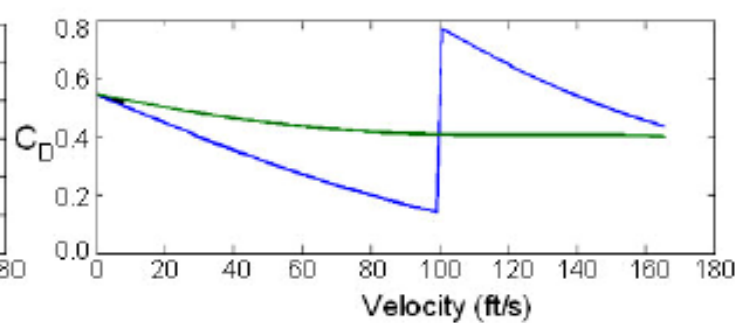

(b)

Figure 6-13: Variations in a) $C_{L}$ and b) $C_{D}$ during Takeoff with Flap Variations

The only major change between these results and those with the varying mass flow was a large increase in $C_{D}$ at the start of the takeoff run which was the source of the 
difference between the two L/D curves. From the figure it can be seen that there is a significant increase in drag with an addition of mass flow regardless of the flap deflection. The final numbers for the varying flap cases can be seen in Table 6 .

Table 6-3: Results for Variable Flap Deflections

\begin{tabular}{|c|c|c|c|c|c|c|c|c|}
\hline & \multicolumn{7}{|c|}{ Speed at which Flap was Deflected to $64^{\circ}$} \\
\hline & & \multicolumn{2}{|r|}{100} & \multicolumn{2}{|r|}{180} & \multicolumn{2}{|c|}{ Quadratic Increase to 180} & \multirow{2}{*}{$\begin{array}{c}\text { Whole Time } \\
\text { actual }\end{array}$} \\
\hline & & actual & $\%$ decrease & actual & $\%$ decrease & actual & $\%$ decrease & \\
\hline $10 \mathrm{~kg} / \mathrm{s}$ & Takeoff Distance (ft) & 2514 & 0.87 & 2441 & 3.75 & 2533 & 0.12 & 2536 \\
\hline Mass Flow & $B F L(f t)$ & 2780 & 1 & 2656 & 5.41 & 2780 & 1 & 2808 \\
\hline $20 \mathrm{~kg} / \mathrm{s}$ & Takeoff Distance (ft) & 2185 & 0.91 & 2069 & 6.17 & 2190 & 0.68 & 2205 \\
\hline Mass Flow & $B F L(f t)$ & 2385 & 2.09 & 2217 & 8.99 & 2387 & 2.01 & 2436 \\
\hline
\end{tabular}

The actual decreases in BFL and takeoff were remarkably similar to those seen with the variable mass flow. The best performance again was found with the later flap deflection with the higher mass flow with roughly a 9\% decrease in BFL. As far as reliability was concerned, the advantage the variable flap has over the variable mass flow could arguably be that only a small $10 \%$ chord length flap has to be deflected instead of large amounts of mass flow being diverted. In either case, it was interesting to find that for the same aircraft, the balanced field length could be shortened by up to $8 \%$ simply by delaying either the dual radius flap deflection or the mass flow out of the ejection slot. 


\section{Interim Model}

As this study progressed, improvements to different aspects of the model were continually being added. Among those improvements were a better thrust model and an improved aerodynamics model. Once these improvements were added, the BFL code was run again in order to generate a figure similar to Figure 6-9. This section will briefly discuss the details of those two improvements and compare these interim results to those presented in the previous section.

\subsection{Improved Thrust Model}

The original thrust model used for the STOL transport for the preliminary results utilized a quadratic variation of thrust with Mach number. The idea was to include the effect of the engines providing less than sea level static thrust as the aircraft increases in speed. The major motivation to improving the fidelity of the thrust model was a recently awarded NASA grant for a design of an $n+2$ generation STOL transport test bed that would investigate different advanced STOL concepts. The current study provided significant framework and methodology that will be used in that research.

In addition, the BFL code used in this study was used in order to implement a higher fidelity engine model which was provided by Professor Mark Watters. Rather than simply assuming the thrust decreased quadratically throughout takeoff, Mr. Waters' model includes many more capabilities. The performance of the engine can be completely determined by specific engine parameters including the rate and state of bleed air. This model provided a tool which could be used to size the primary engines which could provide the required amount of mass flow to power the $\mathrm{CCW}$. In addition, the coupled effect of engine and CC performance could also be modeled with this code. 
However, for the remaining results which will be presented, the improved thrust model was used but the coupling effect was not included. It is the author's hope that future research will incorporate not only the thrust and high lift coupling, but also the effects which both of those complex systems have on controllability.

\subsection{Improved Aerodynamic Model}

The preliminary results were based on a metamodel generated from the first CFD cases. These cases were based of grids generated by Gambit using the uniform volume growth rate which provided extremely high volume growth rates between the cells. Since the rule of thumb was that the growth rate was to be no more than $20 \%$ between cells, it was found that the preliminary aerodynamic model had grid dependent solutions. Obviously the grid dependent solutions needed to be improved in order to provide a more accurate metamodel. To accomplish this end, another series of 37 CFD cases were run using Gambit meshes which had the identical surface mesh as before, but a volume growth rate set to $30 \%$. Although these meshes still lacked boundary layer cells, which made resolving the turbulence difficult, these solutions allowed for the generation of a metamodel with less gridding error which could be used in interim BFL simulations to verify the preliminary results.

In order to determine how many points would be necessary to create the 4-D aerodynamic model, several different error analysis techniques were performed. One example, which is illustrated in Figure 7-1, was a technique developed which used a gradient based optimizer in order to find the maximum amount of error within the model. 


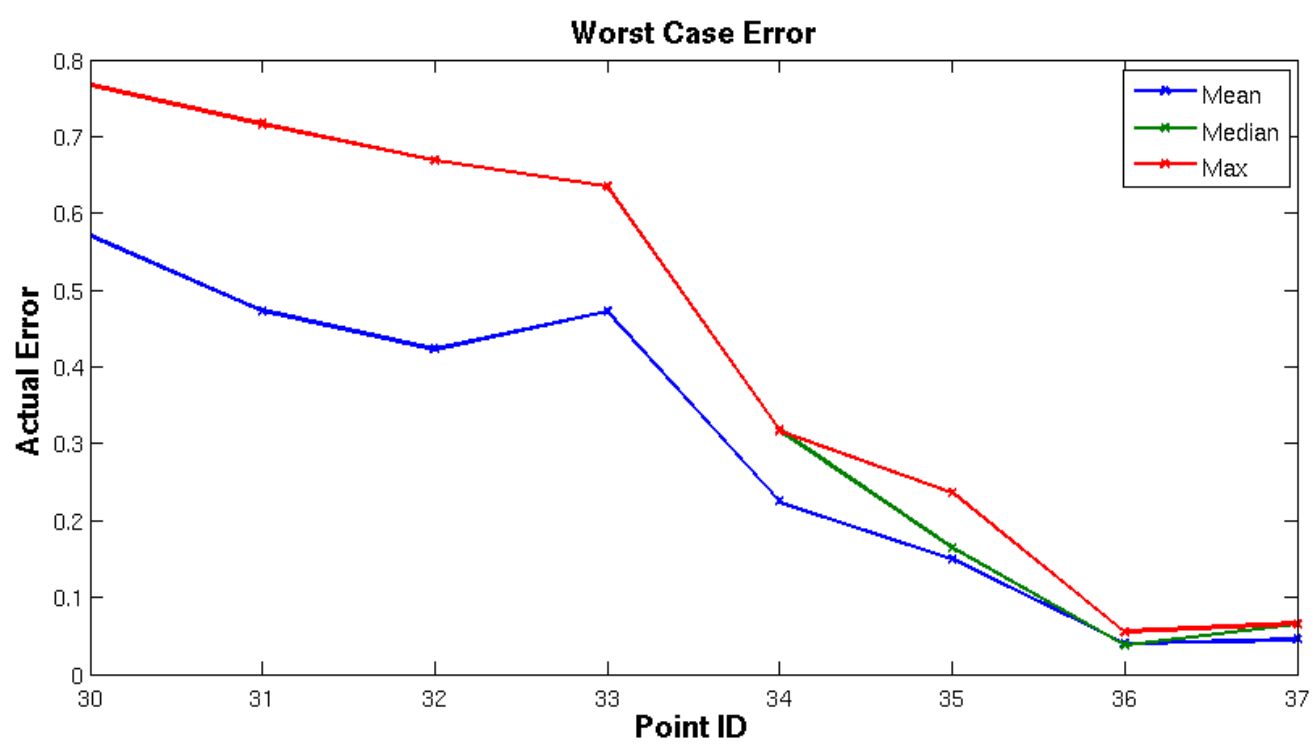

Figure 7-1: Worst Case Error Analysis for $\mathbf{C}_{\mathbf{L}}$

From the figure, it is clear that after 30 CFD cases were added to the model the error began to show a convergence trend which leveled out at between 36 and 37 cases. A similar approach was used in the preliminary model which showed the need of 35-40 cases to define the model. Since the dimensions of two the models were identical, the number of points required to define them were almost identical.

The interim metamodel showed the aerodynamic data with similar trends to the preliminary model throughout the design space. The largest difference between the models was the significant increase in lift and drag throughout the design space. For a Mach number of 0.15 , angle of attack of $5^{\circ}$, mass flow rate of $15 \mathrm{~kg} / \mathrm{s}$, and a flap deflection of $60^{\circ}$ the $C_{L}$ increased by $19.6 \%$ from 2.57 to 3.14 and the $C_{D}$ increased by $11.6 \%$ from 0.382 to 0.437 . There were some subtle differences provided by the higher quality grids. For example, the trend for $\mathrm{C}_{\mathrm{L}}$ as the Mach number decreased as seen in Figure 7-2 and Figure 7-3. 


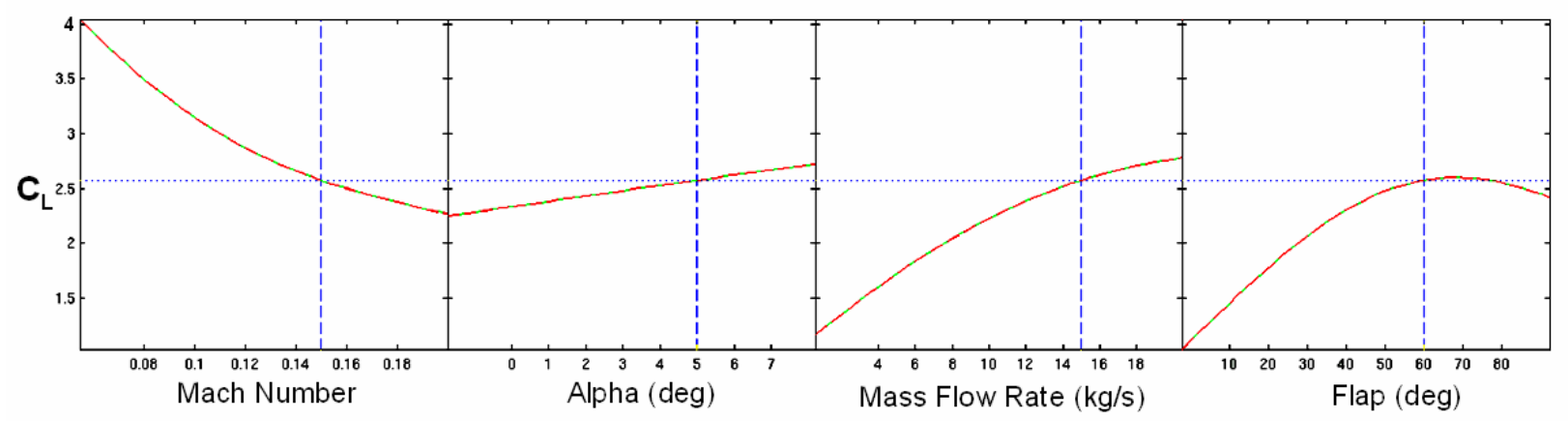

Figure 7-2: Metamodel for CL for the Preliminary Results

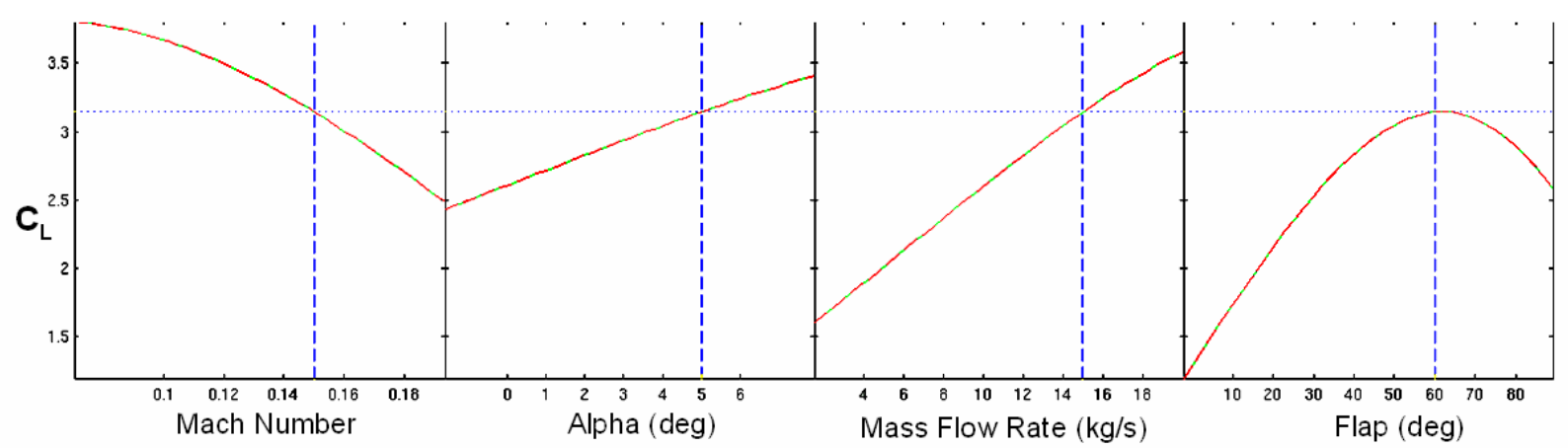

Figure 7-3: Metamodel for CL for the Interim Results

The interim results show the $\mathrm{C}_{\mathrm{L}}$ leveling off at a maximum value while the preliminary results indicated that the lift tended to asymptote towards infinity which does not make physical sense. The same relationship was found with the interim results for $\mathrm{C}_{\mathrm{M}}$ and $\mathrm{C}_{\mathrm{D}}$. Another difference which can be seen from the previous two figures is the rate at which lift decreases after the optimum flap deflection of about $60^{\circ}$. Because the interim results have nearly $20 \%$ more lift, the separation which occurs after $60^{\circ}$ reduces the amount of lift at an even higher rate. As a result, the value of $C_{L}$ at the $90^{\circ}$ flap for the interim model actually gets closer to the same value from the preliminary model. With the major trends verified between the two models compared, the next step was to run the BFL simulation and compare those results. 


\subsection{Interim BFL Results}

With the updated aerodynamics metamodel and the improved thrust model, another sweep of the BFL design space was performed similar to the results seen in section 6.6. The interim BFL results for the STOL transport is shown in Figure 7-4.

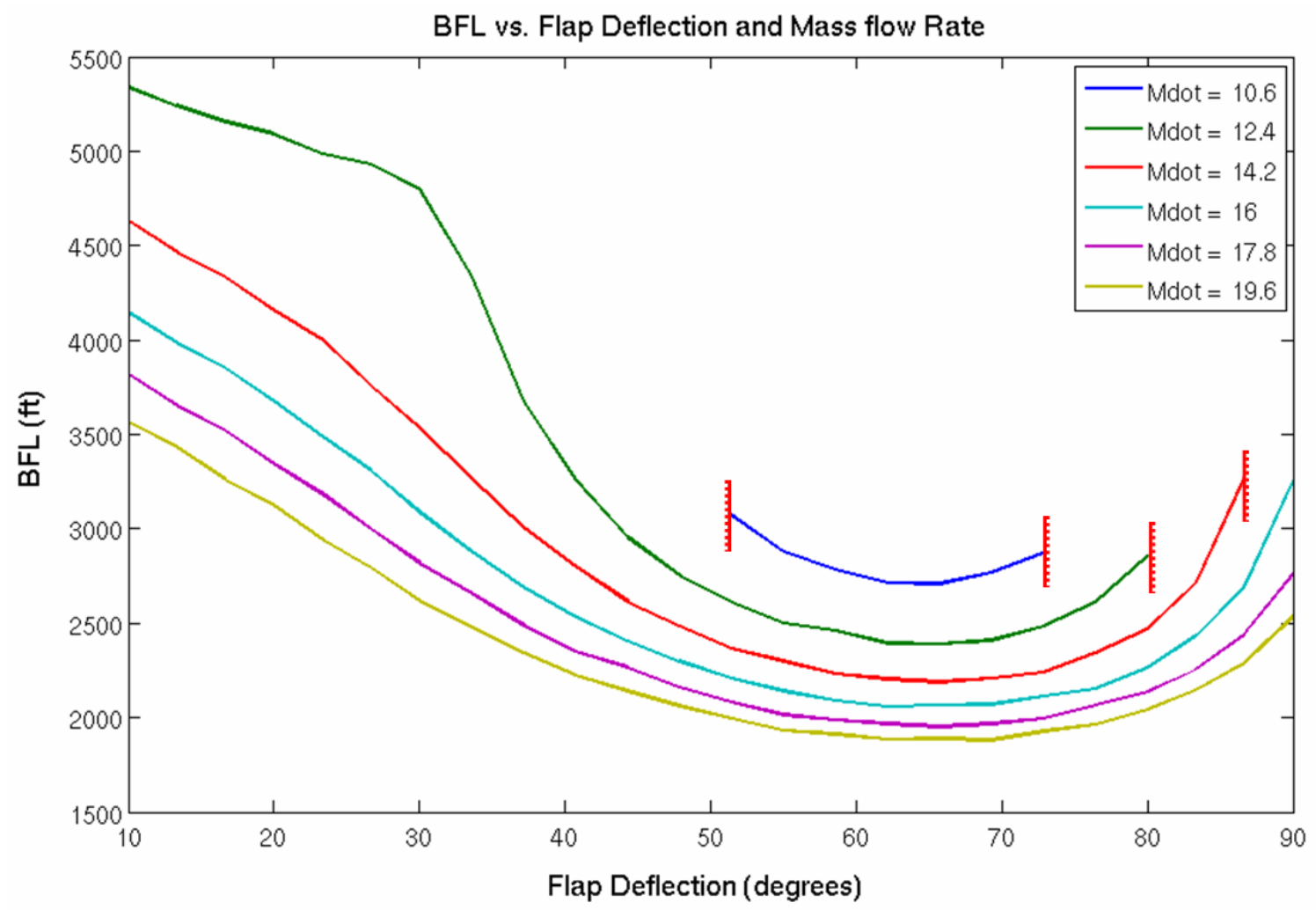

Figure 7-4: Interim BFL Performance

With the updated metamodel, the sweep of the design space was increased to include more of the lower and higher flap deflections in order to obtain a better picture of the BFL performance. It is important to note that the mass flow rates were increased for the interim results because the higher drag limited the transports ability to takeoff at the lower mass flow rates. The most obvious difference which can be seen from these results is the drastic change slop for the BFL contour with a mass flow of $12.4 \mathrm{~kg} / \mathrm{s}$. A similar 
slope change can be seen in the other mass flow rates, but not nearly to the same degree. The metamodeling visualization tool showed not abrupt changes in lift or drag at that mass flow rate and flap setting so it is difficult to ascribe the difference in slope to a coupling effect within the CC aerodynamics. It is possible that at lower mass flow rates that the CC shows a higher dependence on flap deflection at the lower values. However, since achieving the lowest BFL possible is the goal for STOL transports, these results of the lower flap deflections coupled with low mass flow rates were not explored in great detail.

The optimum flap deflection which occurred between $60-64^{\circ}$ agreed well with the preliminary results. Also, the same trend showing the diminishing returns with added mass flow rate appeared in both versions of the results. Some of the added detail from the increased range of flap deflections included some of the constraints to a BFL with CCW. The lowest mass flow rate contour had a constraint on the left due to not achieving enough lift to obtain the $81 \%$ lift to weight ratio which was used to define the rotation point.

On the other end of the contour, the transport was able to rotate but was unable to climb enough to clear the obstacle at the higher flap deflections. This constraint also occurred for the next two mass flow rates at successively higher flap deflections. These constraints provide valuable information for the design of a STOL transport. Clearly, there is a minimum amount of mass flow from the ejection slot which is required before a large transport such as this could takeoff with any length of runway. In addition, the flap deflection and mass flow rate should be coupled in a way in order to stay clear of the previously mentioned constraints. Although these interim results provided a significant 
improvement in the quality of the CFD and therefore the BFL results, the next section will detail how the final results included all of the turbulence from the boundary layer in the model in order to develop the final metamodel and BFL performance results. 


\section{Final Model}

The objective of this study was to provide data points for a model to be used in a takeoff simulation. Although capturing every distinct flowfield phenomenon for each case would have been ideal, for this study the main goal was to obtain reasonable results for $\mathrm{C}_{\mathrm{L}}$ and $\mathrm{C}_{\mathrm{D}}$ at a minimum amount of computation. However, it is also evident that a solution that does not adequately capture the turbulence near the surface of the wing will not produce adequate results for lift and drag. The purpose of this section is to present an additional study on grid dependence to measure the error in the preliminary study due to lack of surface resolution and to provide a higher fidelity model to more accurately predict the minimum BFL based off the trends present the preliminary and interim results.

\subsection{Gridding with Icem}

Although Gambit performed well for all of the grids it produced for the preliminary and interim studies, Icem was found to be far superior in its ability to perform many different meshing techniques. This section discusses the gridding process that was used on the three dimensional test case with a $30^{\circ}$ flap deflection.

One of the capabilities provided with Icem was the ability to use blocking throughout the mesh. Blocking allows for the user to easily grid large sections of structured hexahedral cells quickly. This technique was utilized by placing a bounding box around the CCW and gridding that section with unstructured tetrahedral cells, and then blocking the remaining flowfield with hexahedral cells. This can be seen pictured in Figure 8-1 with the $\mathrm{CCW}$ in the center. The blocking technique allows for a composite grid created from the unstructured mesh near the $\mathrm{CCW}$ and the structured mesh filling the 
flow volume. Figure 8-2 shows the merged tetrahedral and hexahedral grids in a cut plane providing a conformal grid interface. In addition to providing the increased cell qualities of hexahedral cells, the blocking method easily allows for different cell growth rates to be specified in different directions. For example, in this grid, the hexahedral cells were grown at a rate of $6 \%$ aft of the wing in order to capture the turbulent wake while the area forward of the wing grew at $10 \%$. Each of the other three flow directions, above the wing, below the wing, and out from the tip were grown at $6 \%$.

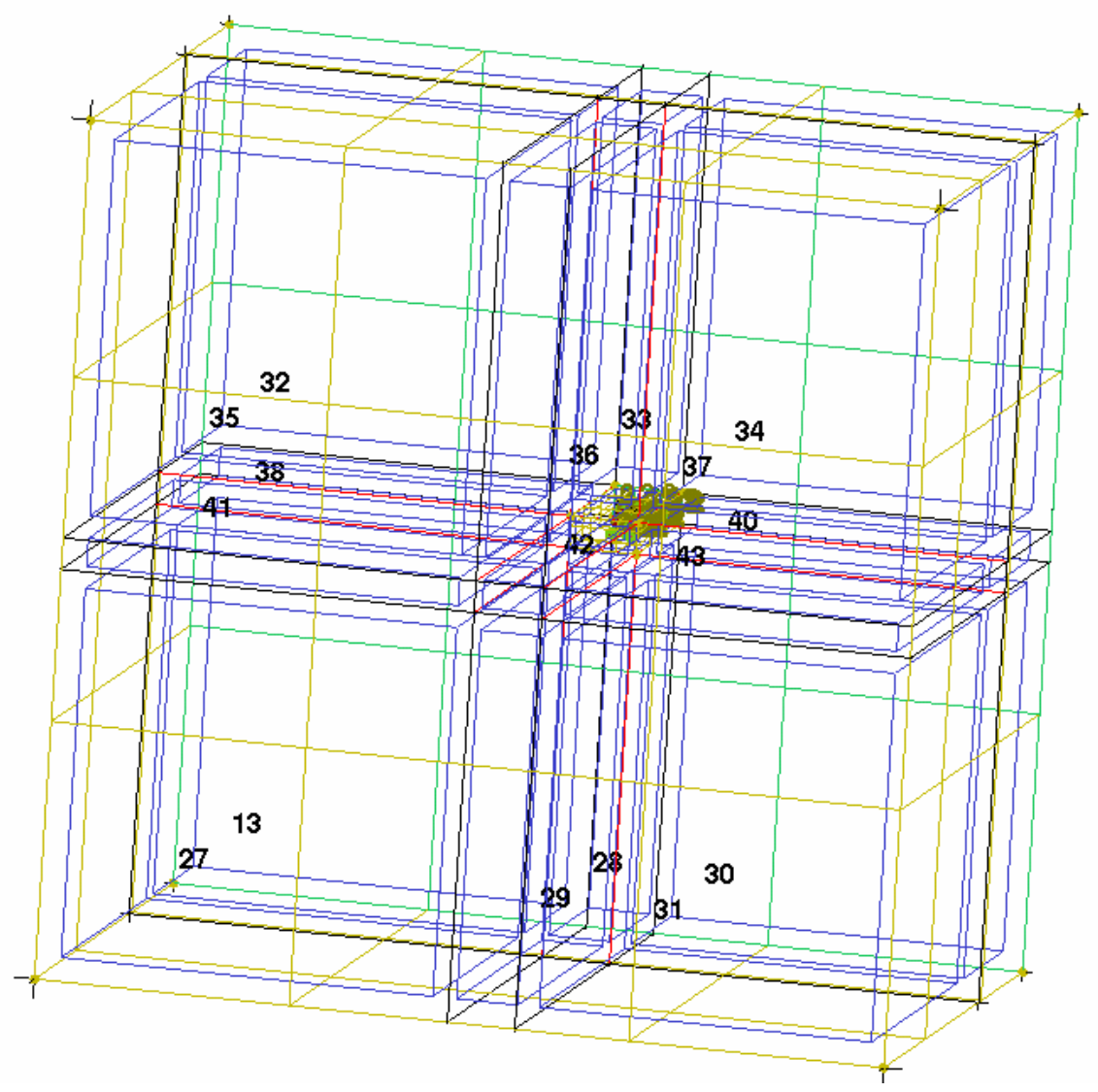

Figure 8-1: Blocking Technique 


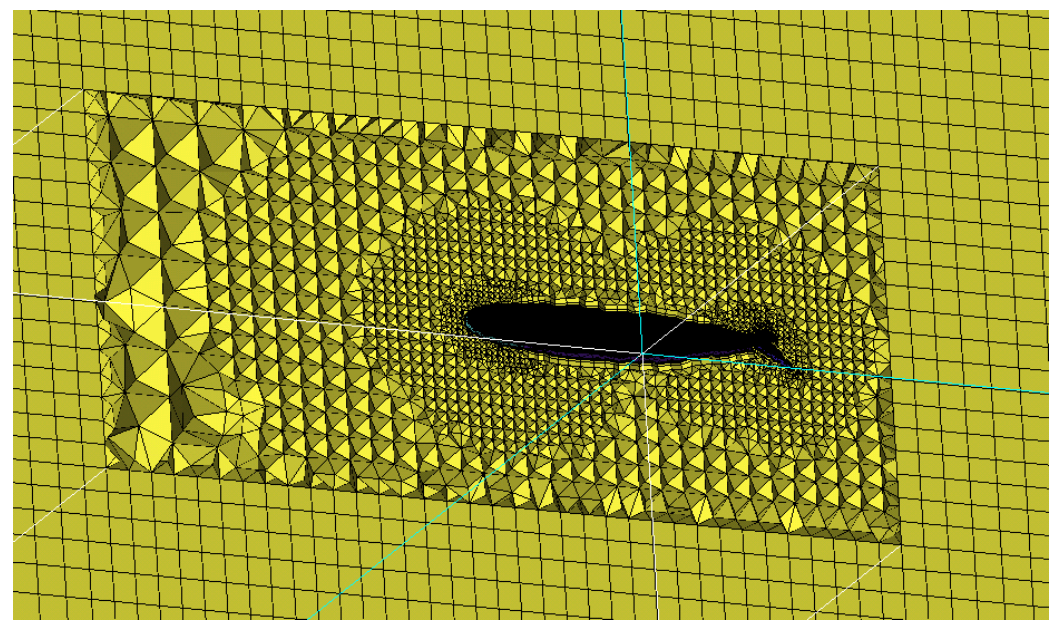

Figure 8-2: Composite Unstructured and Structured Grid

In order to refine areas of interest on the wing, like the jet slot, different parts within Icem needed to be defined along with their maximum edge length allowed. Because there were several different refinement levels on the $\mathrm{CCW}$, several different parts were defined as well as their respective cell and boundary layer parameters. Figure 8-3 shows an example of the setup window where all of these parameters were entered for the different parts.

\begin{tabular}{|c|c|c|c|c|c|c|c|c|c|c|}
\hline Part & Prism & $\begin{array}{l}\text { Hexa } \\
\text { Core }\end{array}$ & $\begin{array}{l}\text { Max } \\
\text { Size }\end{array}$ & Height & $\begin{array}{c}\text { Height } \\
\text { Ratio }\end{array}$ & $\begin{array}{l}\text { Num } \\
\text { Layers }\end{array}$ & $\begin{array}{c}\text { Tetra } \\
\text { Size Ratio }\end{array}$ & $\begin{array}{l}\text { Tetra } \\
\text { Width }\end{array}$ & $\underset{\text { Limit }}{\text { Min Size }}$ & $\begin{array}{c}\text { Max } \\
\text { Deviation }\end{array}$ \\
\hline BIG_BOX & \lrcorner & & 0 & 0 & 0 & 0 & 0 & 0 & 0 & 0 \\
\hline BOX & \lrcorner & & 0 & 0 & 0 & 0 & 0 & 0 & 0 & 0 \\
\hline ENDPLATE & 四 & & 0.04 & 0.00058 & 2.2 & 5 & 1.15 & 0 & 0 & 0 \\
\hline FLAPS_30 & 回 & & 0.08 & 0.00058 & 2.2 & 5 & 1.15 & 0 & 0.005 & 0 \\
\hline FLAPS_30_TINY & 四 & & 0.04 & 0.00058 & 1.9 & 4 & 1.15 & 0 & 0 & 0 \\
\hline GAP_30 & 回 & & 0.06 & 0.00058 & 1.8 & 6 & 1.2 & 0 & 0 & 0 \\
\hline LEADING_EDGE & 四 & & 0.05 & 0.00036 & 2 & 5 & 1.15 & 0 & 0 & 0 \\
\hline SLOT & 回 & & 0.0023 & 0.00036 & 1.4 & 3 & 1.4 & 0 & 0 & 0 \\
\hline SLOT_TOP & 回 & & 0.06 & 0.00036 & 2.2 & 5 & 1.15 & 0 & 0 & 0 \\
\hline STAT_FLAPS & 四 & & 0.07 & 0.00036 & 2.2 & 5 & 1.15 & 0 & 0 & 0 \\
\hline SYMMETRY & \lrcorner & & 0 & 0 & 0 & 0 & 0 & 0 & 0 & 0 \\
\hline TINY_SLOT_TOP & 可 & & 0.04 & 0.00036 & 1.9 & 4 & 1.15 & 0 & 0 & 0 \\
\hline WING & 回 & & 0.18 & 0.00058 & 2.2 & 6 & 1.15 & 0 & 0 & 0 \\
\hline AIR_INNER & 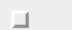 & च & 0.0 & & & & & & & \\
\hline
\end{tabular}

Figure 8-3: Boundary Layer and Tetrahedral Growth Parameters 
In the middle of this window is a column labeled "Tetra Size Ratio" which specifies the growth rate of tetrahedral cells away from the specified surface. To ensure that there were enough cells on the wing surfaces and the volume, these rates were all set to grow at $15 \%$ with the exception of the cells adjacent to the jet slot which were set to $40 \%$ on account of there very small size. Although the $40 \%$ growth at the slot helped to reduce the number of cells in that region, all of the other surfaces with their $15 \%$ rates dominated the $40 \%$ rate and thus maintained proper cell volume ratios throughout the volume.

One of the significant advantages Icem had over Gambit was in its ability to grow boundary layer cells in three dimensions. Boundary layer cells are not only good at capturing the turbulence at the surface, but they are also very efficient in terms of total cell count. The same amount of surface resolution provided by purely tetrahedral cells would require an exponentially greater number of cells. Icem had many options for the boundary layer cells, including the initial cell height, the growth rate, and the total number of cells. One of the objectives in gridding is to ensure proper cell volume ratios between neighboring cells and this was accomplished with the prism boundary layer cells using a spreadsheet like the one pictured in Figure 8-4.

\begin{tabular}{|r|r|r|r|r|}
\hline \multirow{2}{*}{\begin{tabular}{l} 
Max Cell Edge \\
\cline { 3 - 5 } Ratio
\end{tabular}} & \multicolumn{2}{|r|}{0.08} & & \\
\cline { 3 - 5 } & & 2.2 & Maximum Vol & 0.00006 \\
\hline Cell & Height & Top Cell Vol & max AR & Volume Ratio \\
\hline 1 & 0.0005800 & 0.000002 & 137.93 & 37.54 \\
\hline 2 & 0.0012760 & 0.000004 & 62.7 & 17.06 \\
\hline 3 & 0.0028072 & 0.000008 & 28.5 & 7.76 \\
\hline 4 & 0.0061758 & 0.000017 & 12.95 & 3.53 \\
\hline 5 & 0.0135868 & 0.000038 & 5.89 & 1.6 \\
\hline 6 & 0.0298911 & 0.000083 & 2.68 & 0.73 \\
\hline 7 & 0.0657603 & 0.000182 & 1.22 & 0.33 \\
\hline 8 & 0.1446728 & 0.000401 & 0.55 & 0.15 \\
\hline & & & & \\
\hline
\end{tabular}

Figure 8-4: Prism Volume Transition Spreadsheet 
Using this spreadsheet, the proper number of prism layers could be calculated to ensure a reasonable volume transition between the top prism cell and its neighboring tetrahedral. For this test case, the initial boundary layer height was set to 0.00058 meters (or $0.58 \mathrm{~mm}$ ) on the wing surfaces to obtain a target $\mathrm{y}^{+}$value of 50 . With that initial cell height, maximum edge size and growth rate within the boundary layer were modified until the volume transition to the tetrahedral cell was reasonable. Since the maximum edge length was used for these calculations, the actual volume ratios for a majority of the surface would be lower then the value predicted by the spreadsheet. A cut plane of the boundary layer as it turns around the jet slot can be seen in Figure 8-5.
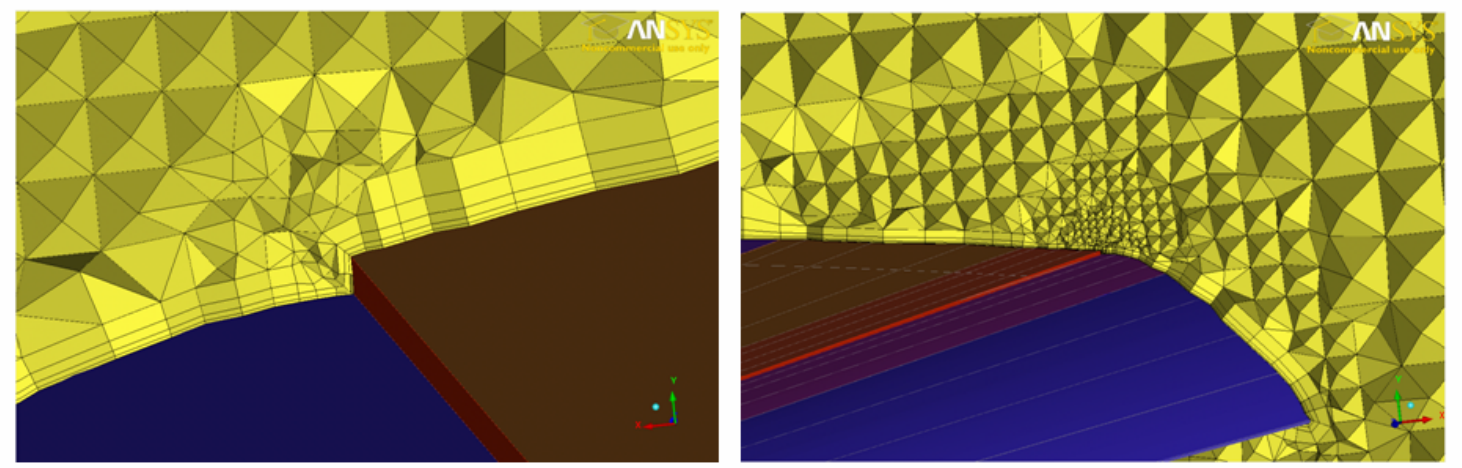

Figure 8-5: Boundary Layer Cells Turning Around Jet Slot

Several different iterations were required to determine the correct number of boundary layer cells in order to best resolve the flow without requiring highly skewed tetrahedrals to be grown from the top layer. In Figure 8-5, the left image shows highly skewed cells adjacent to the top boundary layer cells at the slot. More surfaces were added so that the cells could be refined in this specific area and also reduce the number of prism layers which is pictured on the right. This same procedure was repeated in several problem areas on the $\mathrm{CCW}$, such as the flap junction pictured in Figure 8-6, until a good balance between boundary layer cells and skewed tetrahedral cells could be achieved. 


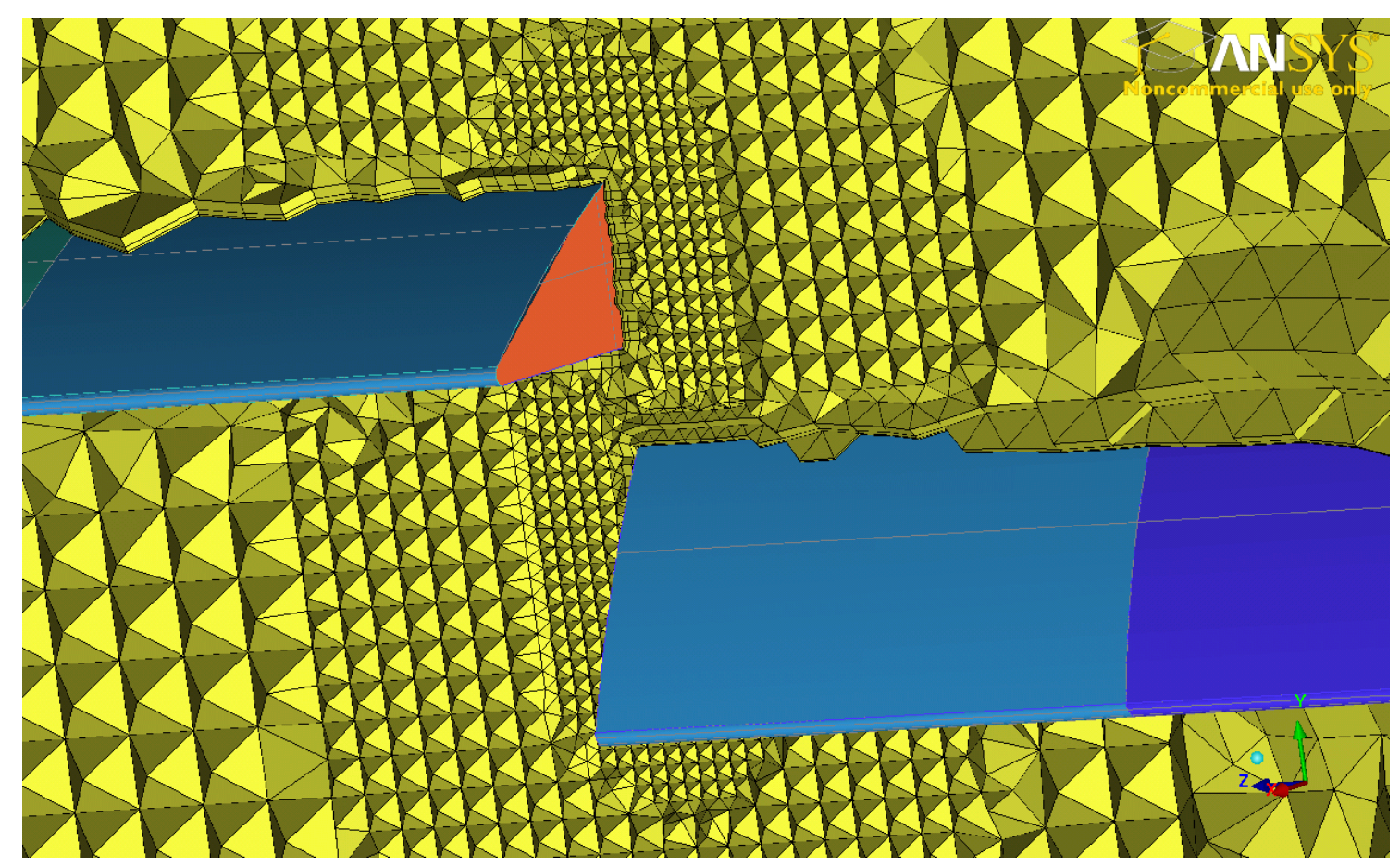

Figure 8-6: Refinement and Boundary Layer Cells at Flap Junction

The three major areas on the wing which needed the most resolution were the leading and trailing edges as well as the region near the ejection slot. Since the ejection slot was so small to begin with, resolving that region affected the mesh density of many of the other surfaces on the wing. The slot was meshed with an unstructured grid, 3 to 4 cells high and over 1,000 cells long. This produced triangular cells with low skewness and also resulted in triangular prisms which extruded from the slot surface.

With the density at the slot determined, all of the surfaces near the slot were then meshed with specific attention given to cell skewness and volume transition. The basic process involved entering different cell maximum cell edge lengths into the table shown in Figure 8-3 until a compromise between the number of cells required to adequately define the CCW geometry and the cell quality. If a greater number of small cells were 
used to define a surface, it was much easier to produce high quality tetrahedral and boundary layer cells. However, since there is an upper limit on the amount of computational power available, a grid with an infinite number of cells was obviously not possible which thus led to the compromise.

Several regions, specifically near the slot, required a great attention to detail in order to obtain quality boundary layer cells. Two methods exist in Icem in which the number of layers of boundary layer cells can be specified. First, an automated method existed in which a maximum cell aspect ration was defined along with a maximum number of layers. The second method simply involved specifying the number of layers for each of the surfaces which were defined for the wing. For this research, it was found that a combination of both methods allowed for the best grids. For a majority of the meshing, the wing was simply split into its major features, like the leading edge, slot, and flap. Once they were defined as separate surfaces the meshed proceeded as previously mentioned. Some additional care was needed at the areas where some of the larger wing surfaces neared the ejection slot. Since the cells in those highly refined regions were much smaller than the surrounding wing surfaces, a number of smaller surfaces needed to be defined to ensure a proper transition between the boundary layer cells from the different surfaces. Such transition surfaces can be seen in Figure 8-5 and Figure 8-6. At these transition surfaces, the number of boundary layer levels was reduced from the larger wing surfaces and raised from the more refined slot areas. In this way, the boundary layer would not gain or lose too many layers at one time and cause poor cell quality. 
Another feature provided with Icem was the ability to convert the volume within the bounding box around the $\mathrm{CCW}$ to hexahedral cells. Although tetrahedral cells work well at forming complex geometry, they also introduce error due to the faces not being aligned with the freestream flow. The cell conversion feature within Icem called hexacore converts 12 tetrahedral cells into a single hexahedral cell and significantly reduces the number of cells within the inner block. The surface cells and interface cells remained as tetrahedrals in order to define the complicated geometry, but the majority of the inner block volume was converted to hexahedral cells as can be seen pictured in Figure 8-7.

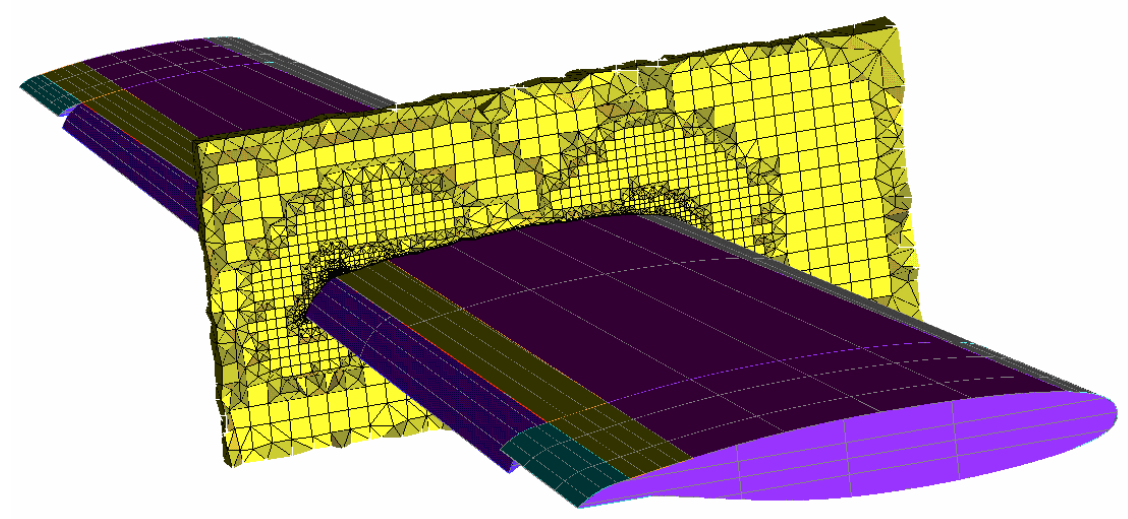

Figure 8-7: Cut-plane Displaying Hex-Core Conversion

Icem proved to be the superior gridding software when it came to refining the boundary layer and hexahedral blocking techniques. The final grid produced using Icem contained just fewer than 10 million cells throughout the flow volume and can be seen in Figure 8-8. How this highly refined grid performed in comparison with its counterparts generated in Gambit will be presented next. 


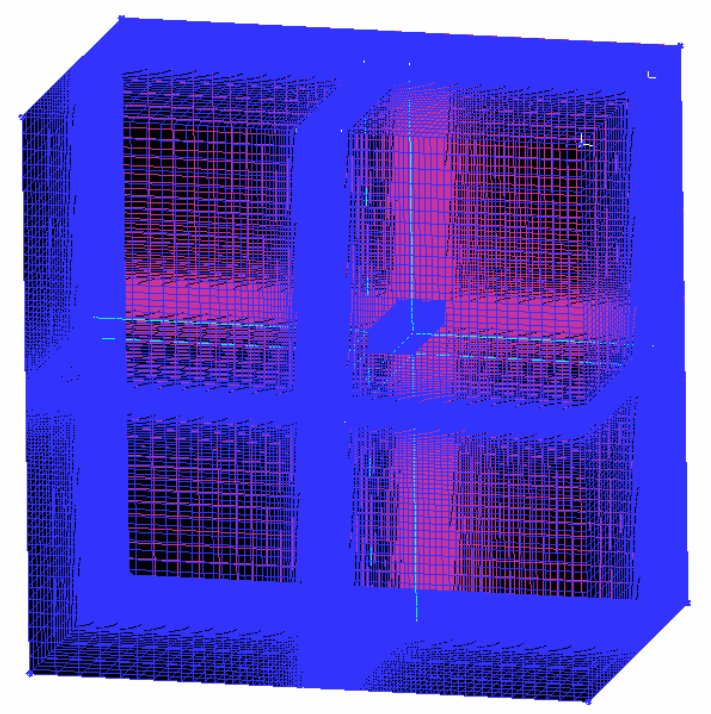

Figure 8-8: Final Mesh for 30 Degree Flap

\subsection{Solution Comparisons and Grid Improvements}

This section compares the interim results from the Gambit grids to the more refined Icem grids in order to determine how to correctly resolve the boundary layer as well as error estimations due to discritization. In addition, a comparison of the surface and flowfield properties will be presented.

\subsubsection{Grid Resolution}

One of the major sources of error in a CFD study has to do with the quality of the grid. Even if a solution converges, poor grid quality can alter the solution and provide erroneous results. This section will present results from using grids of various sizes and examine the variations in the results. However, a more detailed grid independence study will follow in section 8.2.3.

This grid study used a case with the following parameters: Mach number of 0.081 , angle of attack of $5.7863^{\circ}$, mass flow rate of $5.1928 \mathrm{~kg} / \mathrm{s}$, and a flap deflection of $30^{\circ}$. 
This case had already been run in the interim model and was run using the refined $30^{\circ}$ flap deflection grid created in Icem. Two variations of the Icem grid were also used in this comparison. The first variation was used to verify if a higher growth rate could be used within the CCW bounding box which would reduce the number of cells from 10 million to 3.5 million. The fine Icem grid employed a $15 \%$ growth rate within the bounding box while the coarse version of the Icem grid utilized the default growth rate of 1.5. The higher growth rate was significantly higher than the 1.2 rule of thumb and therefore required verification. The third variation to the Icem grid utilized hexacore conversion to the fine Icem grid in order to discover if the reduced number of cells provided by the hexacore grid could produce the same results. A summary of the results for the different grids is provided in Table 8-1.

Table 8-1: Grid Type and Resolution Results

\begin{tabular}{|l|c|c|c|c|c|}
\hline Volume Rate (\%) & $\begin{array}{c}\text { Number } \\
\text { of Cells }\end{array}$ & $\mathbf{C}_{\mathbf{L}}$ & $\mathbf{C}_{\mathbf{D}}$ & $\mathbf{C}_{\mathbf{M}}$ & $\begin{array}{l}\% \mathbf{C}_{\mathbf{L}} \text { Difference } \\
\text { from RE }\end{array}$ \\
\hline Gambit (Uniform) & $1.2 \times 10^{6}$ & 1.83 & 0.187 & 0.453 & 7.35 \\
\hline Gambit (30) & $1.73 \times 10^{6}$ & 1.9475 & 0.1684 & 0.47915 & 1.40 \\
\hline Icem (15) Hex-Core & $7.44 \times 10^{6}$ & 1.9386 & 0.16786 & 0.48157 & 1.86 \\
\hline Icem Default & $3.49 \times 10^{6}$ & 1.9189 & 0.16492 & 0.4735 & 2.85 \\
\hline Icem (15) & $9.88 \times 10^{6}$ & 1.9563 & 0.16755 & 0.48842 & 0.96 \\
\hline
\end{tabular}

The last column the table expresses the numerical error due to the grids based on a Richardson Extrapolation (RE). A RE is a method for obtaining a higher-order estimate of the continuum value, which is the value at which the cell spacing is zero (i.e. an infinite number of cells). The basic equation for the RE is given as $\mathrm{Eq}(19)$.

$$
f_{h=0}=f_{1}+\frac{f_{1}-f_{2}}{r^{p}-1}
$$


The values for $f_{l}$ and $f_{2}$ are for the solution functional values, which for this study was $\mathrm{C}_{\mathrm{L}}$, $r$ is a function of the refinement ratio between the two grids, and $p$ is a function of the order of accuracy of the solution. The RE was based on the three Icem grids since those grids used similar gridding methods rather than including the Gambit grids composed entirely of tetrahedral cells. For these results, the RE will provide at a minimum a thirdorder estimate for the continuum value of $\mathrm{C}_{\mathrm{L}}$.

The first interesting result that can be seen from Table 8-1 is that the 30\% Gambit mesh with an error of only $1.40 \%$ surprisingly performed better than two of the more refined Icem meshes with 1.86 and $2.85 \%$ errors respectively. If these results were correct with all three grids providing results with less than $3 \%$ discritization error, the obvious choice for an additional higher fidelity experiment would be the $30 \%$ Gambit grids since it had less error than the hexacore grid with only $16.2 \%$ of the required cells. However, an additional piece of information was provided in the flowfield comparisons which determined that the drag obtained by the Gambit grids were actually $10 \%$ lower than that obtained with the highly refined Icem case. This discrepancy in viscous drag calculations as well as flowfield comparisons between the Gambit and Icem meshes will be addressed in the next section which will then lead to the grid independence study and the final results.

\subsubsection{Flowfield Comparisons and the Boundary Layer}

In addition to providing accurate results for the integrated values, it was also important to ensure that the important flowfield properties were also present. Four different comparisons of the more refined Gambit and higher fidelity Icem results will be 
presented in this section: the $C_{P}$ surface and flowfield distributions, velocity contours, the wake capture, and finally a comparison of the surface $\mathrm{y}^{+}$.

The $C_{P}$ distributions over the wing and in the surrounding flowfield show the regions that generate the highest lift. Figure 8-9 shows the contours on the $\mathrm{CCW}$ with a cut-plane near the wing tip.
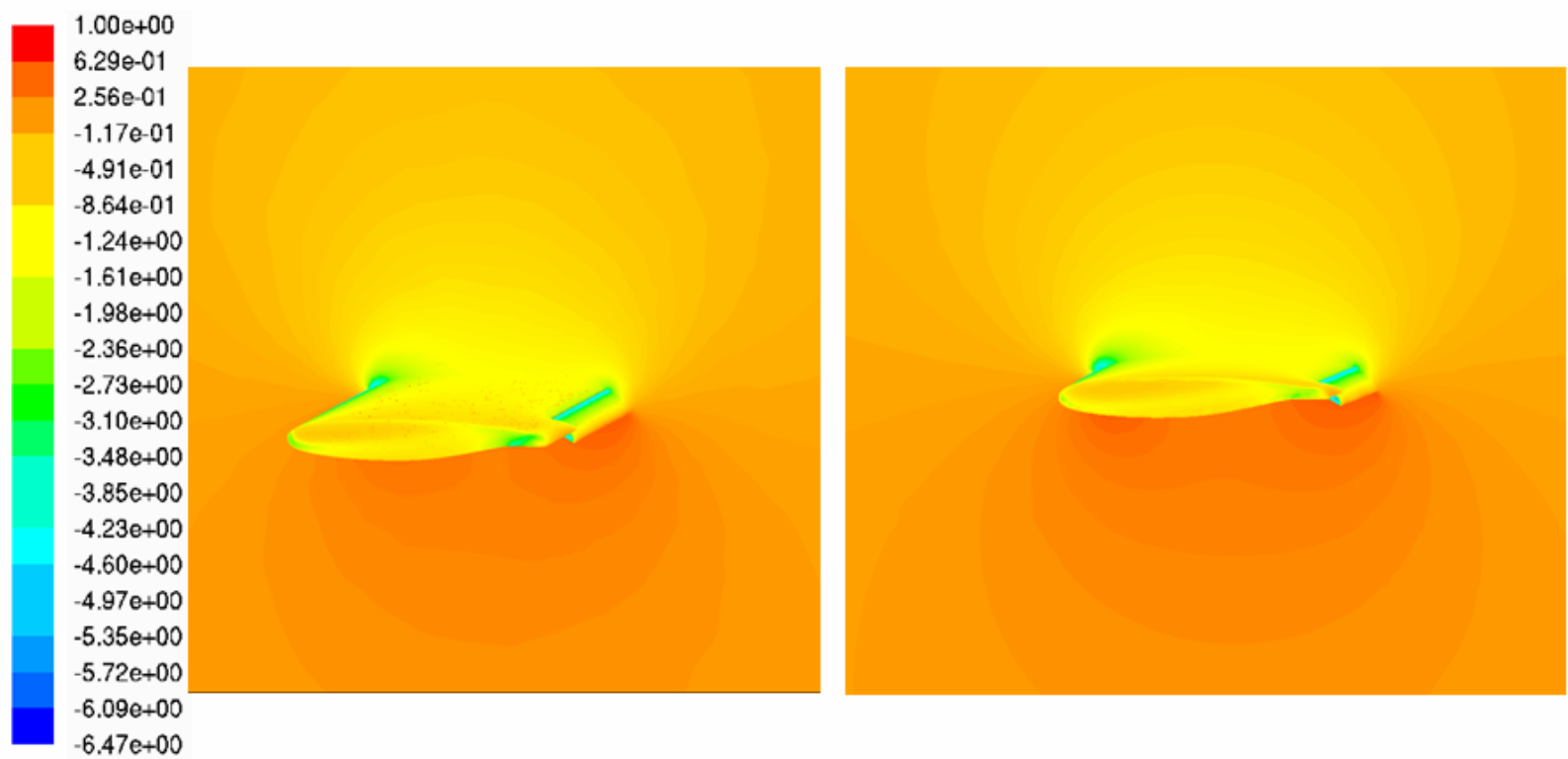

Figure 8-9: $\mathrm{C}_{\mathrm{p}}$ Contours for left: Gambit Mesh and right: Icem Mesh

The contours are remarkably similar for the two grids despite the eight million cell difference. The contours not only have the same levels, but also the same elliptical shapes. Also, the region with the highest $\mathrm{C}_{\mathrm{P}}$ occurs under the $\mathrm{CC}$ flap for both cases. Some of these similarities can also be seen in Figure 8-10 where the cut-plane was created perpendicular to the previous example. 


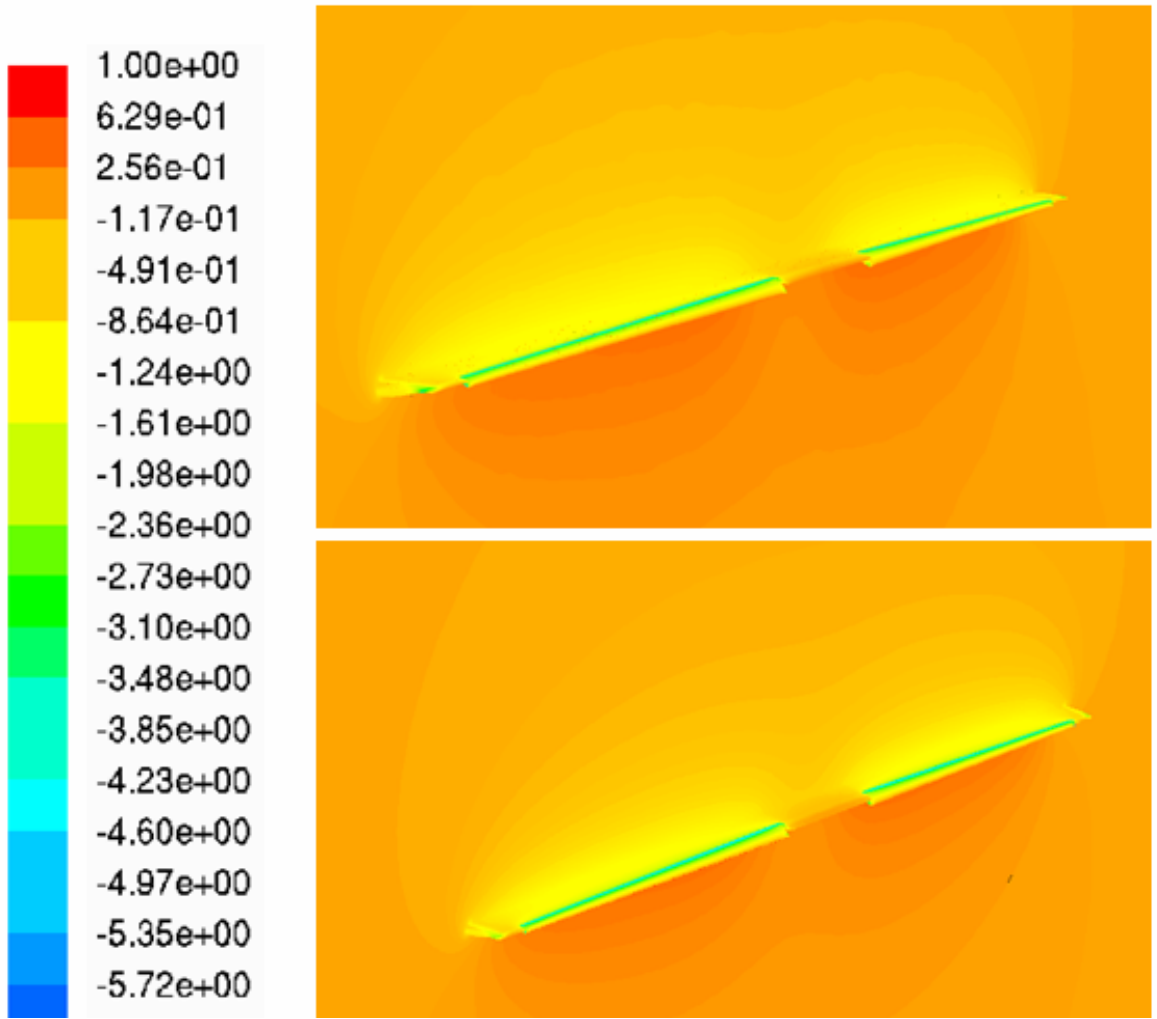

Figure 8-10: $\mathrm{C}_{\mathrm{p}}$ Contours for top: Gambit Solutions and bottom: Icem solution

Again, the two solutions appear to produce very similar contours surrounding the CCW. Both solutions show a dip in pressure in the center region without the $\mathrm{CC}$ flap. However, this contour does show a subtle difference with the Gambit solution. Upon close inspection, it can be seen that the outer contour lines appear to be slightly more jagged than the well refined contours from the Icem solution. This was attributed to the much higher cell growth rate which was used for the Gambit grid.

In addition to the $\mathrm{C}_{\mathrm{P}}$ distribution, the velocity contours in the flowfield also show some of the similarities and differences in the resolution of the two cases. The first difference was the low velocity region aft of the flap where the higher fidelity solution shows a smaller separation region. As with the results presented in section 6.5 , the freestream flow appeared to be separating from the high speed flow out of the ejection 
slot and this effect was slightly reduced in the Icem solution. This discrepancy between the results was mainly due to the higher resolution of the shear layer between these flows which allowed for the higher fidelity grid to better predict the separation point.

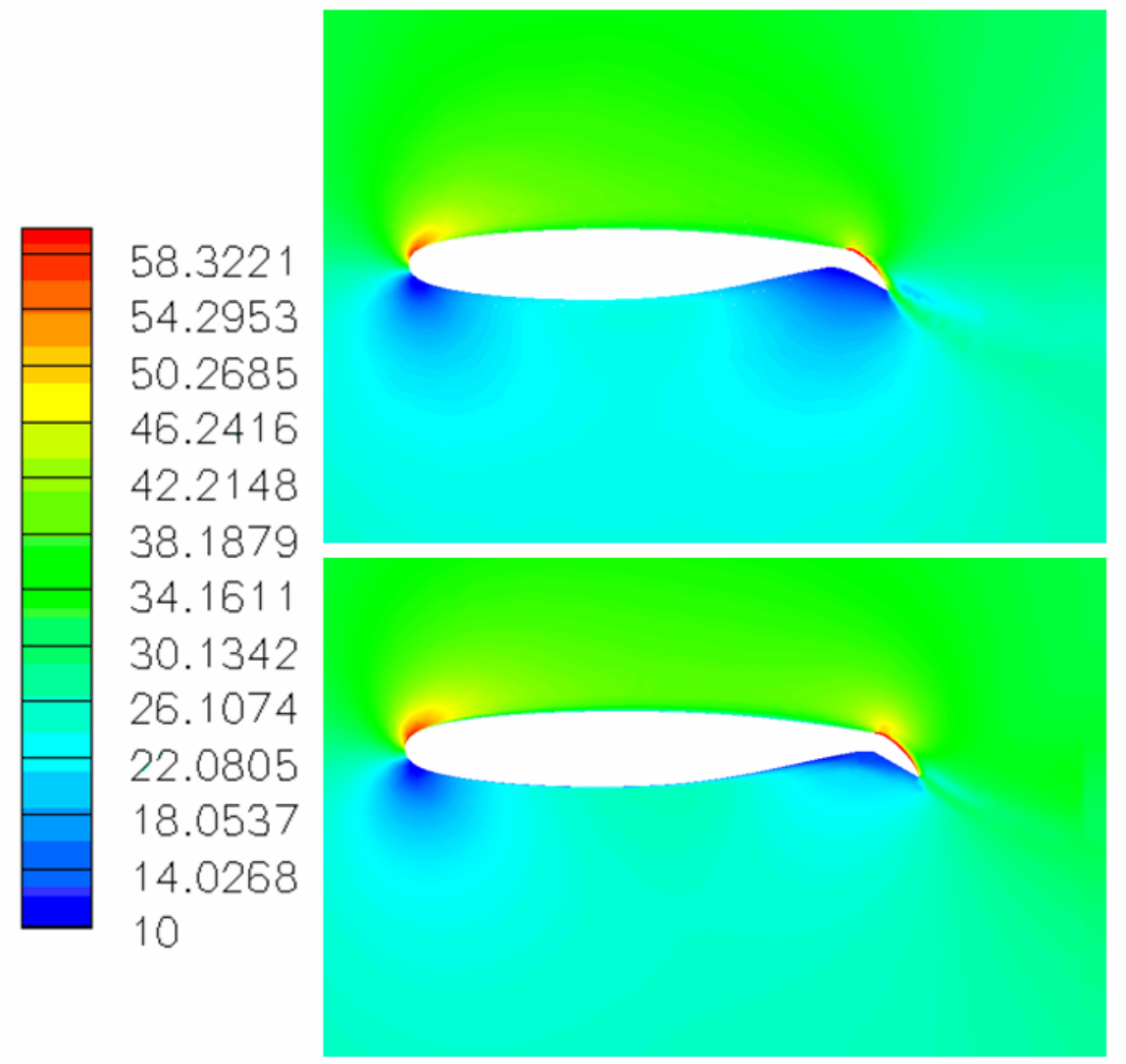

Figure 8-11: Velocity Contours of top: Gambit and bottom: Icem Solutions (m/s)

An additional difference which is easier to see was the larger recirculation region underneath the flap for the Gambit solution. The Icem solution showed a much smaller stagnation region which again can be attributed to the better resolution of the boundary layer turbulence.

The third comparison was for the ability of the meshes to capture the wake generated by the CCW. Currently it is still up for debate as to whether or not resolving 
the wake actually affects the quality of the results for lift and drag. For this study, a significant difference in wake resolution can be seen in Figure 8-12.

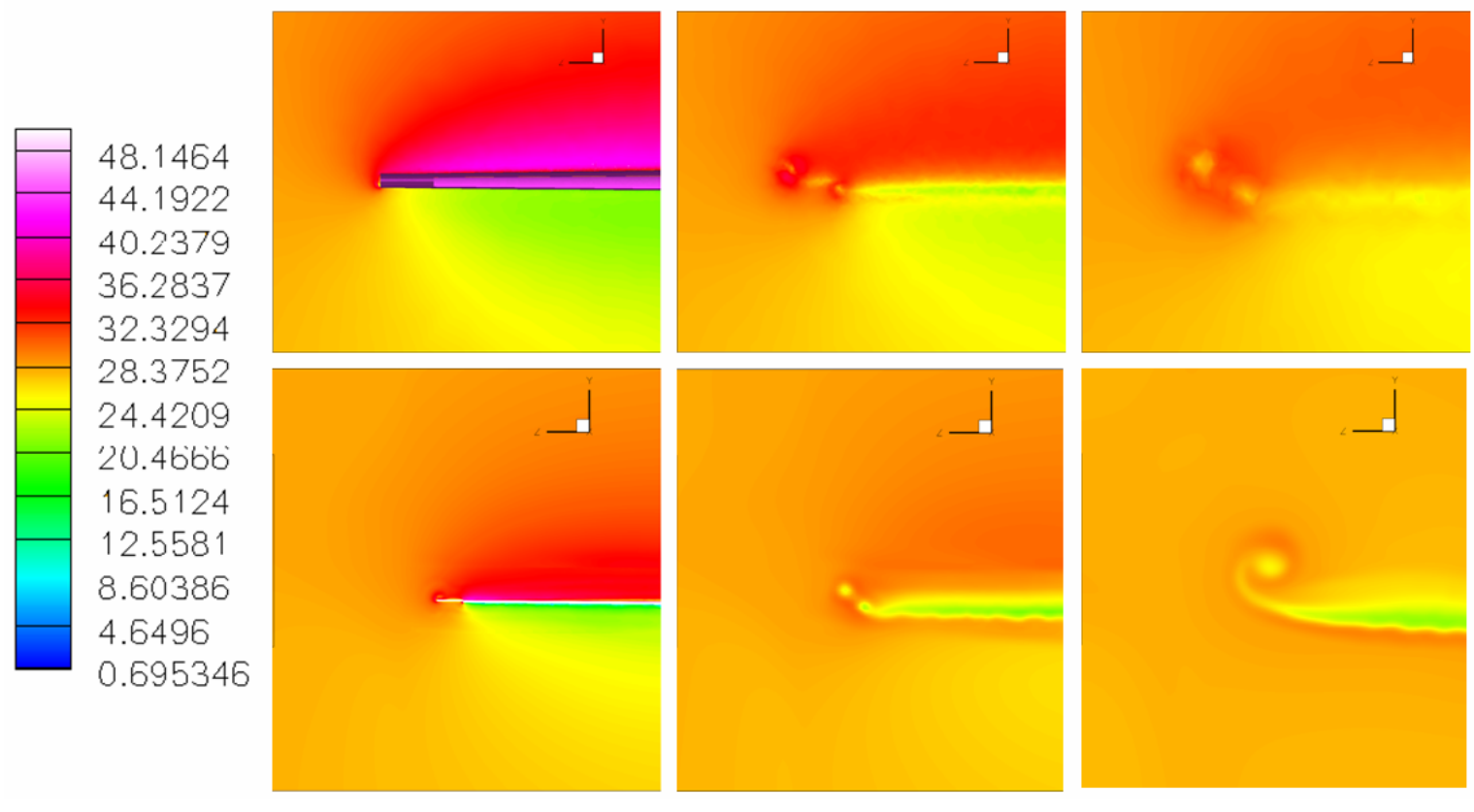

Figure 8-12: Velocity Slices (m/s) of the wake for top 3: Gambit, bottom 3: Icem

Figure 8-12 shows velocity magnitude slices as they progress downstream of the wing. The slices on the far left show the velocity of the flow at the aft end of the wing while the other two sections to the right are slices progressively farther downstream. Several key differences between the two solutions were apparent. First, the wake was clearly better defined for the more resolved Icem solution. The two major vortices shed at the wing tip and at the tip of the $\mathrm{CC}$ flap were much clearer in the Icem solution and propagated all the way to the end of the computational domain while the fuzzy vortices in the Gambit solution diffused into the freestream within a few meters past the wing.

The next comparison that will be given is the surface $\mathrm{y}^{+}$results obtained from the two solutions which can be seen in Figure 8-13. It is clear from these results that the surface resolution was far superior with the Icem solution. This was to be expected 
because of the lack of boundary layer cells in the Gambit mesh. However, the refinement level of the Icem grid was actually found to be too high for standard wall functions which is apparent in the darkest blue regions which fell below the $\mathrm{y}^{+}$of $30 \log$ layer lower boundary.

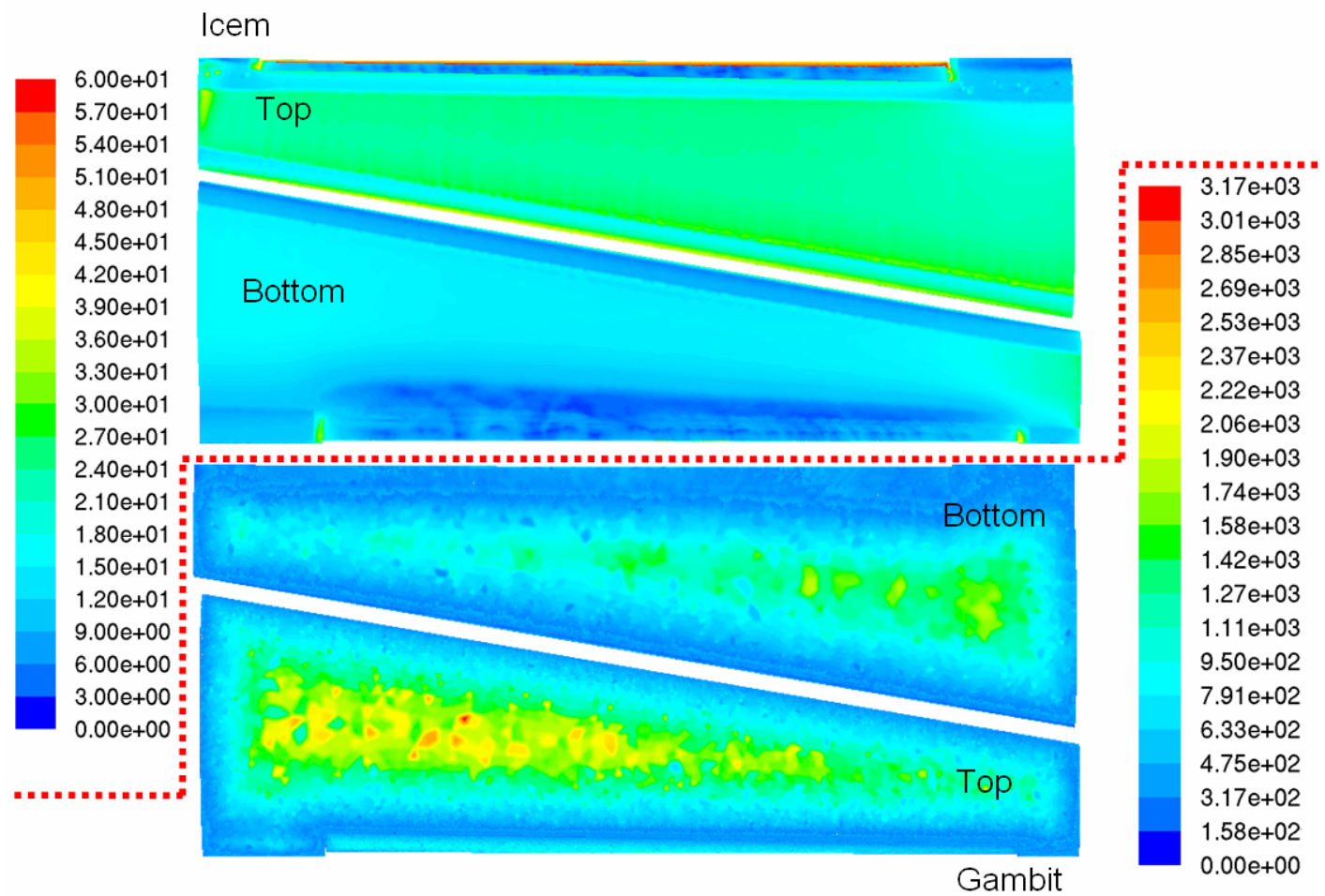

Figure 8-13: Contours of $\mathrm{Y}^{+}$- Icem vs. Gambit

This over refinement for standard wall functions added some error to the solution because for best results with the Spalart-Allmaras model $^{24}$, either a very fine near-wall mesh spacing should be used (on the order of $\mathrm{y}^{+}=1$ ) or a mesh spacing such that $\mathrm{y}^{+}>30$. To show exactly what percentage of cells were within this region, a histogram was created which is shown in Figure 8-14. 


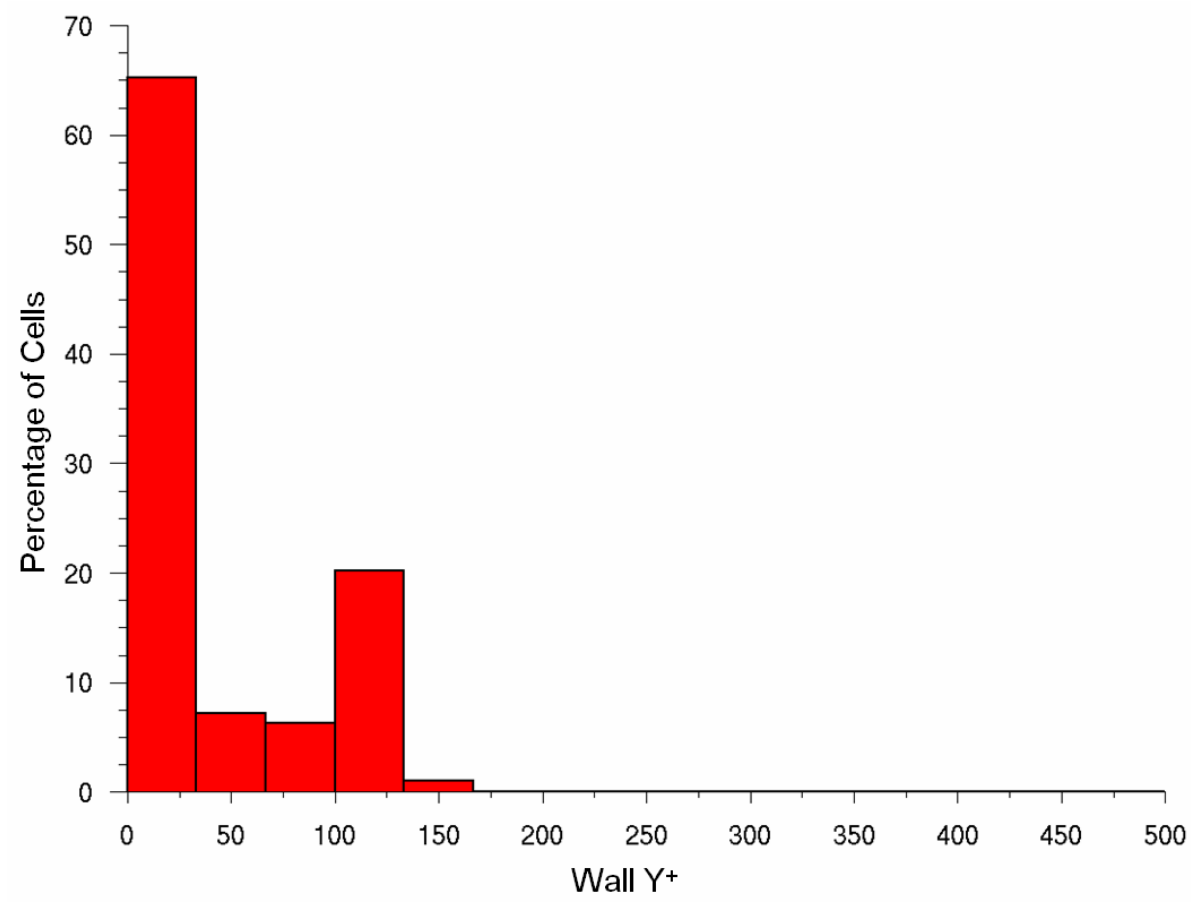

Figure 8-14: Histogram of surface $\mathrm{Y}^{+}$for refined Icem Solution

Although the contour plot gave the indication that a significant portion of the wing had $\mathrm{y}^{+}$ values outside the buffer region, the histogram indicates that $65 \%$ of the cells lie within it. In order to verify if the over refinement significantly impacted the results, two additional grids were created with higher initial cell heights in the areas shown to be over refined in the previous results.

In order to create the grids with better surface $\mathrm{y}^{+}$values, two attempts were made. First, the initial heights of the boundary layer cells were scaled up for the regions that were over refined. The result of this was that most of the wing surfaces were outside the buffer region except for the large recirculation area underneath the $60^{\circ}$ flap. This led to the second attempt in which the wing surfaces which contained cells both inside and outside the buffer region were isolated according to the contours from Figure 8-13 in order to raise the initial cell height and produce $\mathrm{y}^{+}$which would fall outside the buffer 
region. The $\mathrm{y}^{+}$value was assumed to vary linearly with cell height in these regions. With this assumption, the new initial cell heights were determined by scaling up the previous heights.

The two different modified grids were used in the test case as follows: Mach number of 0.15 , angle of attack of $5^{\circ}$, mass flow rate of $15 \mathrm{~kg} / \mathrm{s}$, and this time a flap deflection of $60^{\circ}$. The first attempt resulted in many fewer cells within the buffer region, but enough to warrant additional considerations which led to the second attempt. Contours of surface $\mathrm{y}^{+}$for both attempts can be seen in Figure 8-15.

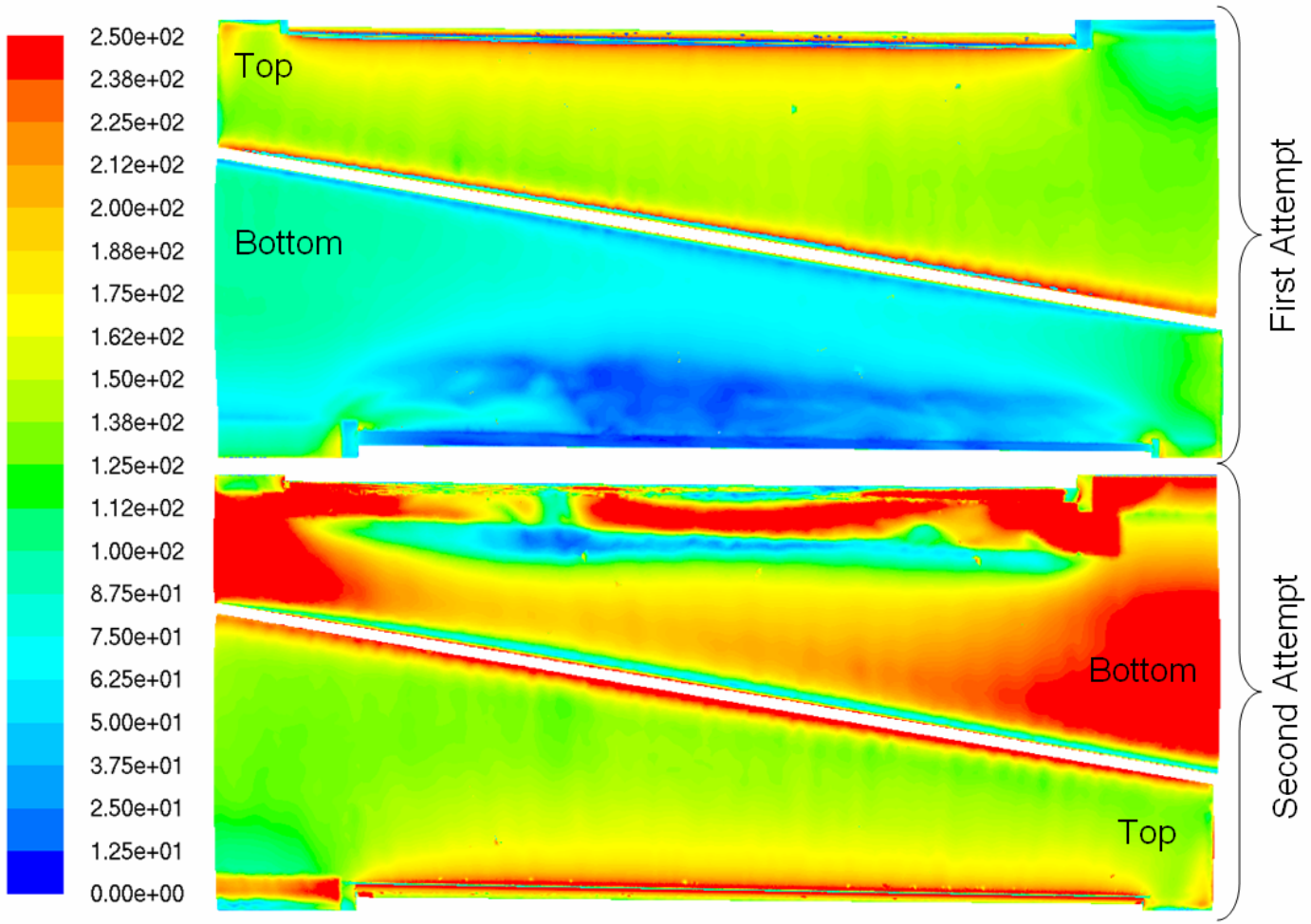

Figure 8-15: Contours of Surface $\mathrm{Y}^{+}$with a $60^{\circ}$ flap

The upper surface of the wing remained largely the same between the two attempts while there were some major differences on the lower surfaces. Clearly the region underneath the flap required additional work to ensure the $\mathrm{y}^{+}$was high enough to provide good turbulence calculations. The large recirculation region significantly 
reduced the velocity of the flow which in turn required a larger initial cell height to maintain proper $\mathrm{y}^{+}$values. The boundary layer cells in this region ended up being very large compared to the rest of the wing and it was found that they were not necessary at all on the lower edge of the flap. It can also be seen that the initial cell heights can actually be scaled down slightly as indicated by the large amount of red seen on the bottom surface of the second attempt. However, 250 was still a reasonable $\mathrm{y}^{+}$value and therefore its solution was taken to be accurate. Histograms showing the surface $y^{+}$value can be seen in Figure 8-16 and Figure 8-17.

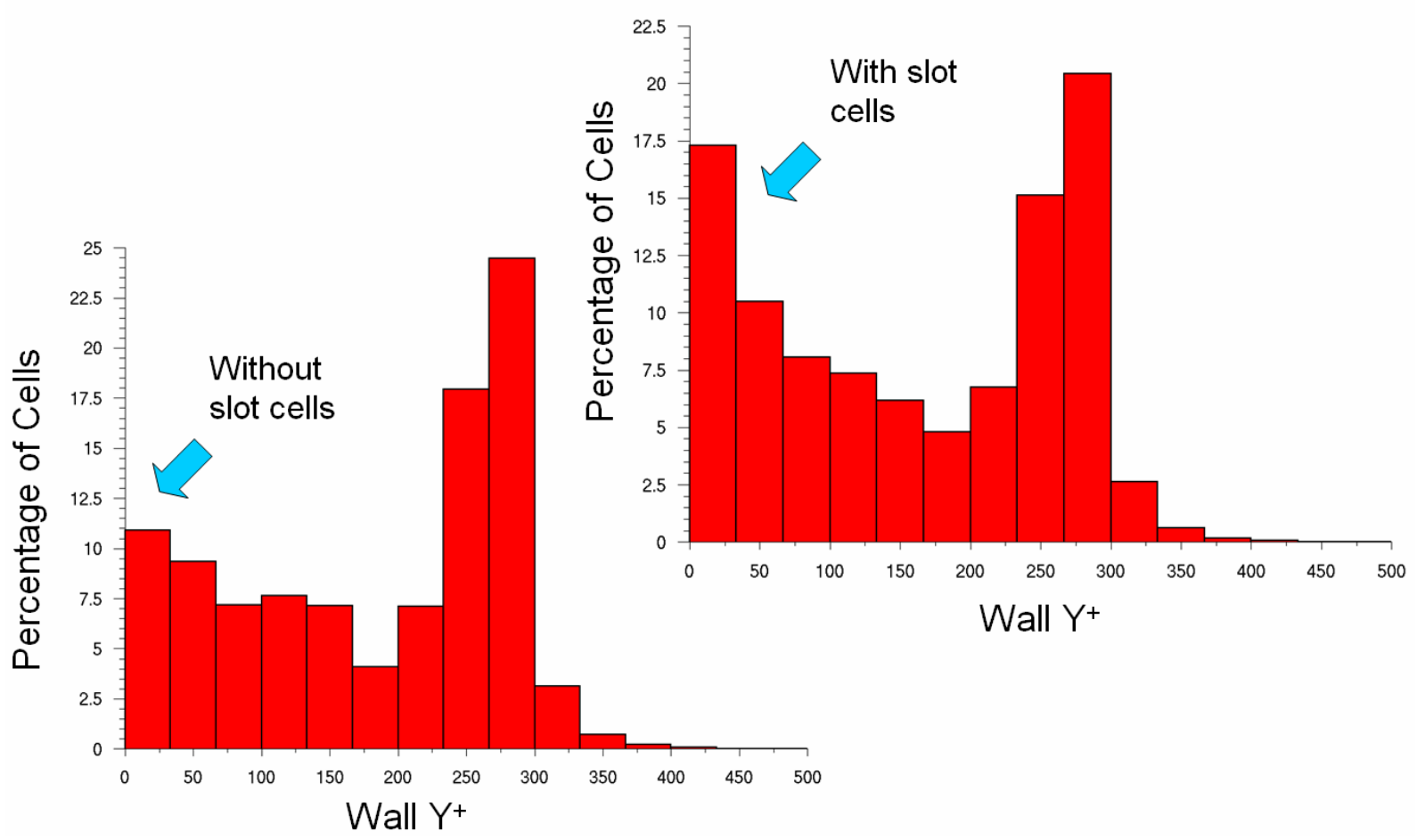

Figure 8-16: Histogram of First Attempt to Achieve Good Y+ Values 


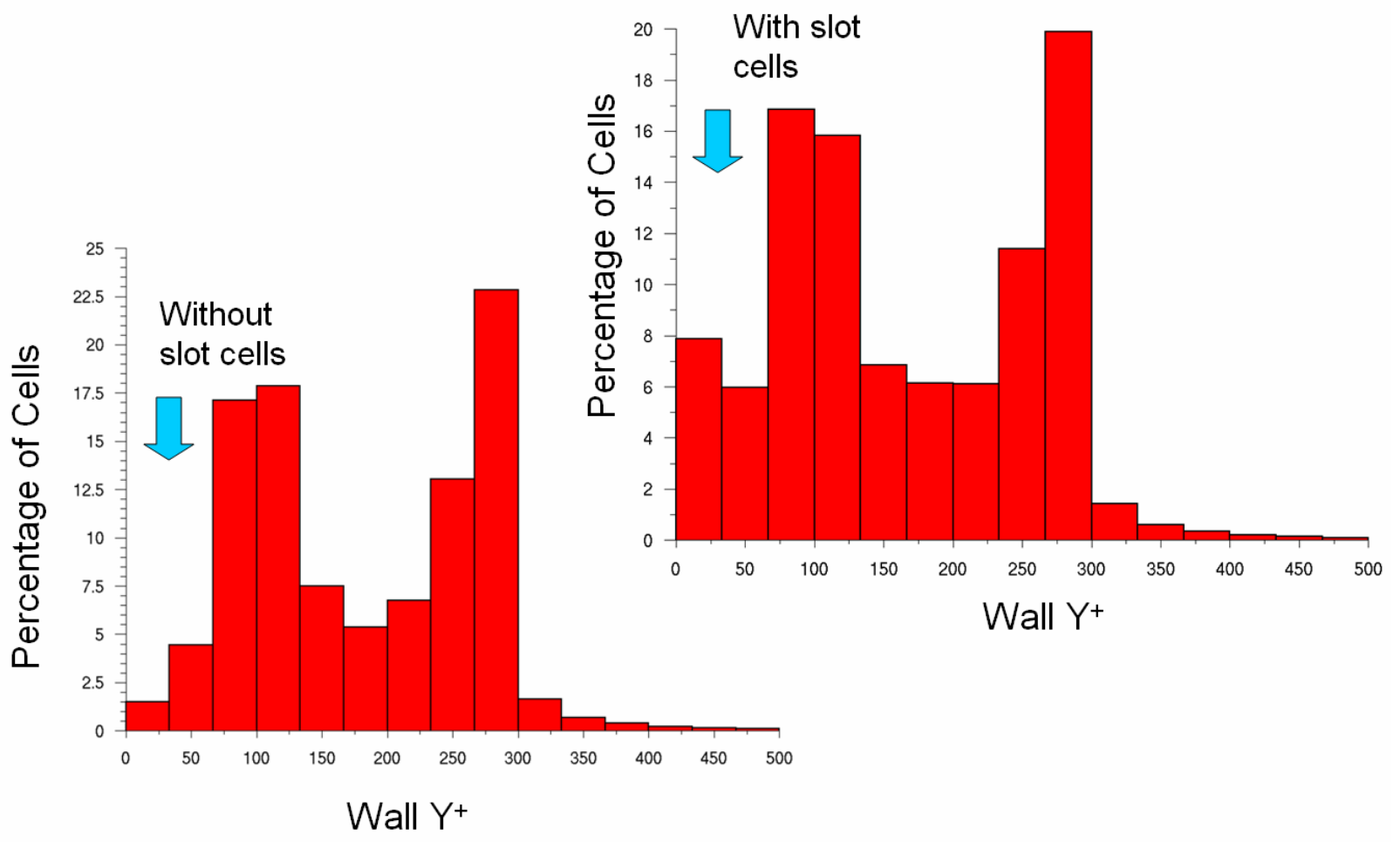

Figure 8-17: Histogram of Second Attempt to Achieve Good Y+ Values

The effect of the over-resolved stagnation region can also be clearly seen from the histograms. It is important to note that two histograms for each solution were included with and without the slot cells included. The slot was the most difficult area to grid and it was determined that since the slot was such a small feature $(0.5 \mathrm{~cm}$ in height $)$ with respect to the wing surface area, increasing the initial cell height for the slot was very difficult and unnecessary. The amount of turbulence error introduced by such a small feature was deemed to be insignificant. With those cells ignored, the percentage of cells within the buffer region decreased from about $11 \%$ for the first attempt to just over $1 \%$ for the second. The overall results for lift and drag for these two cases along with the $30 \%$ Gambit equivalent can be seen in Table 8-2. 
Table 8-2: Surface $\mathrm{Y}^{+}$Results

\begin{tabular}{|l|c|c|c|c|c|}
\hline Volume Rate (\%) & $\mathbf{C}_{\mathbf{L}}$ & $\mathbf{C}_{\mathbf{D}}$ & $\mathbf{C}_{\mathbf{M}}$ & $\begin{array}{c}\% \mathbf{C}_{\mathbf{D}} \\
\text { Difference }\end{array}$ & $\begin{array}{c}\% \mathbf{C}_{\mathbf{L}} \\
\text { Difference }\end{array}$ \\
\hline Gambit $(30)$ & 2.6779 & 0.35109 & 0.83716 & 9.08 & 0.42 \\
\hline Icem $(15)-1^{\text {st }}$ Try & 2.6870 & 0.38699 & 0.84653 & 0.21 & 0.45 \\
\hline Icem $(15)-2^{\text {nd }}$ Try & 2.6667 & 0.38619 & 0.84563 & n/a & n/a \\
\hline
\end{tabular}

The results from this table finally show how the addition of the boundary layer cells alters the value for $C_{D}$. Since a majority of the drag for this high lift wing was induced drag, these results show that once the $\mathrm{y}^{+}$values were within the proper range for the turbulence model, the total drag increased by only $9.08 \%$. However, this much of an increase in viscous drag would have a significant impact on the BFL performance. Therefore, based on these results, one final metamodel was created to produce a final BFL performance plot.

\subsubsection{Grid Independence}

Since a majority of the error for a CFD solution originates from the quality of the grid, it is very important to perform a grid independence study before the results can be used for performance calculations. In section 8.2.1, a grid resolution study was performed on grids which led to grids with a higher quality boundary layer resolution as was just presented. With the boundary layer cells finally performing well at predicting lift as well as drag, a grid independence study was performed to determine how many cells would be required to produce accurate results.

Since a very fine grid of over 8.5 million cells was created for the $\mathrm{y}^{+}$analysis from the previous section, only two additional grids needed to be generated and solved to complete the grid independence study. Like the grid resolution study, the volume growth rate into and off of the surfaces was varied using two methods: the default growth method 
and a growth rate of 1.4. However, unlike the grid resolution study, the grids were now known to be free from errors due to boundary layer resolution leaving discritization error to be determined by the grid independence study. The three grids with drastically different cell counts were generated and the results of their solutions can be seen in Table 8-3 and Figure 8-18. A Richardson Extrapolation was performed in order to predict the $\mathrm{C}_{\mathrm{L}}$ which would be obtained with an infinite number of cells and therefore quantify the amount of discritization error within the three grids.

Table 8-3: Grid Independence

\begin{tabular}{|c|c|c|c|c|}
\hline Number of Cells & $\mathbf{C}_{\mathbf{L}}$ & $\mathbf{C}_{\mathbf{D}}$ & $\mathbf{C}_{\mathbf{M}}$ & \% $\mathbf{C}_{\mathbf{L}}$ Difference from $\mathbf{R E}$ \\
\hline $3.70 \times 10^{6}$ & 2.6192 & 0.37487 & 0.82438 & 1.8438 \\
\hline $5.76 \times 10^{6}$ & 2.6592 & 0.39505 & 0.84042 & 0.3447 \\
\hline $8.95 \times 10^{6}$ & 2.6667 & 0.38619 & 0.84563 & 0.0637 \\
\hline
\end{tabular}

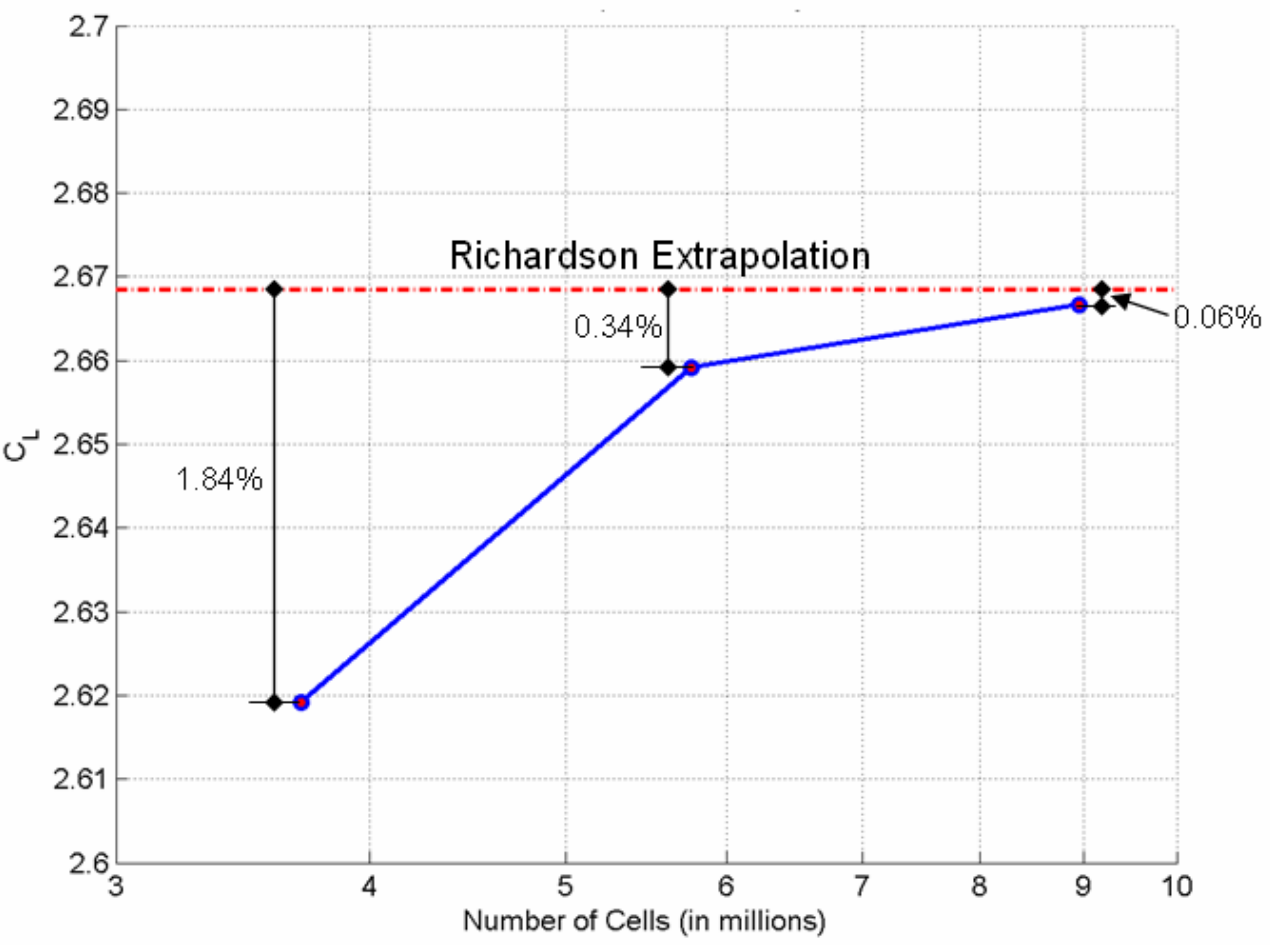

Figure 8-18: Grid Independence Study 
Figure 8-18 clearly shows a convergence trend towards the Richardson Extrapolation value of 2.6684. Another important conclusion that can be made from these results is that the addition of 5.25 million cells only reduces the discritization error by $1.78 \%$. Because creating a metamodel requires the use of many CFD cases, it becomes impractical to use the extra fine grid. As a result, for the final model the CFD cases were based off of solutions obtained using the 3.7 million cell grid which was used in this grid independence study. The following sections will describe the final aerodynamic model, examine the flowfield characteristics, and finally use the model to perform additional BFL calculations.

\subsection{Final Aerodynamic Model}

The goal of the final model was to build upon the previous results and provide an accurate model at an optimum flap deflection. Since both the preliminary and interim results showed an optimal flap deflection between $60-64^{\circ}$, the final higher fidelity results were based off of a single flap deflection of $60^{\circ}$. The grid that was used was based off of the second attempt at correctly resolving the boundary layer which from the previous section was shown to have only a $1.84 \%$ discrepancy from RE continuum result. This grid was found to provide an excellent compromise between exact results and computation time.

However, before the final aerodynamic model will be presented, it is important to once again examine the important features in the flowfield. The first results were from a case with a Mach number of 0.21 , an angle of attack of $6^{\circ}$, a mass flow rate of $17.1 \mathrm{~kg} / \mathrm{s}$, and the $60^{\circ}$ flap deflection which was used for all of the final cases. That case resulted in a $\mathrm{C}_{\mathrm{L}}$ of 2.47 , a $\mathrm{C}_{\mathrm{D}}$ of 0.324 and a $\mathrm{C}_{\mathrm{M}}$ of 0.6294 . One of the properties that clearly 
illustrates such high lifts from a CCW is pressure coefficient, the contours of which can be seen in Figure 8-19.

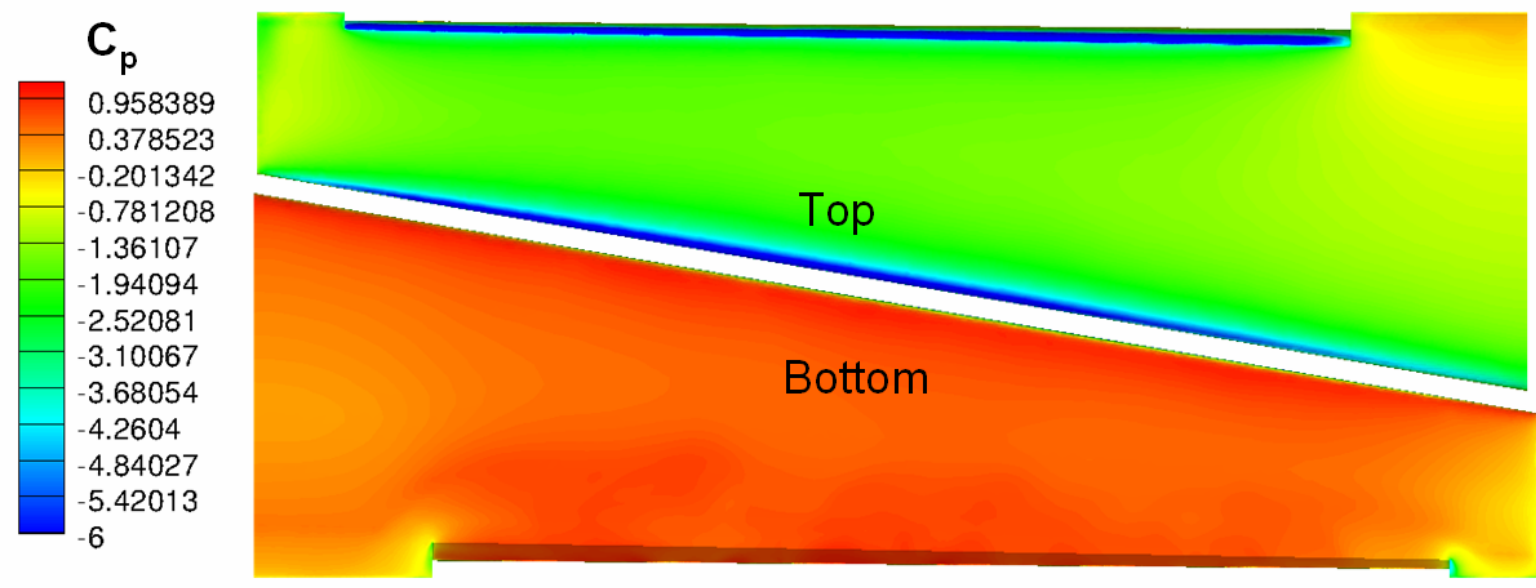

Figure 8-19: Surface $C_{p}$ Contours

From the figure the extreme negative $C_{P}$ values on the upper surfaces of the leading edge and CCW flap can be seen as well as the very high pressure located on the lower surface. The highest and lowest pressures for the lower and upper surfaces respectively primarily fell within the regions of the wing which had the dual radius flap. To illustrate the span-wise variation of $\mathrm{C}_{\mathrm{P}}$, the values at several cross sections were extracted at the locations shown in Figure 8-20 and are illustrated in Figure 8-21.

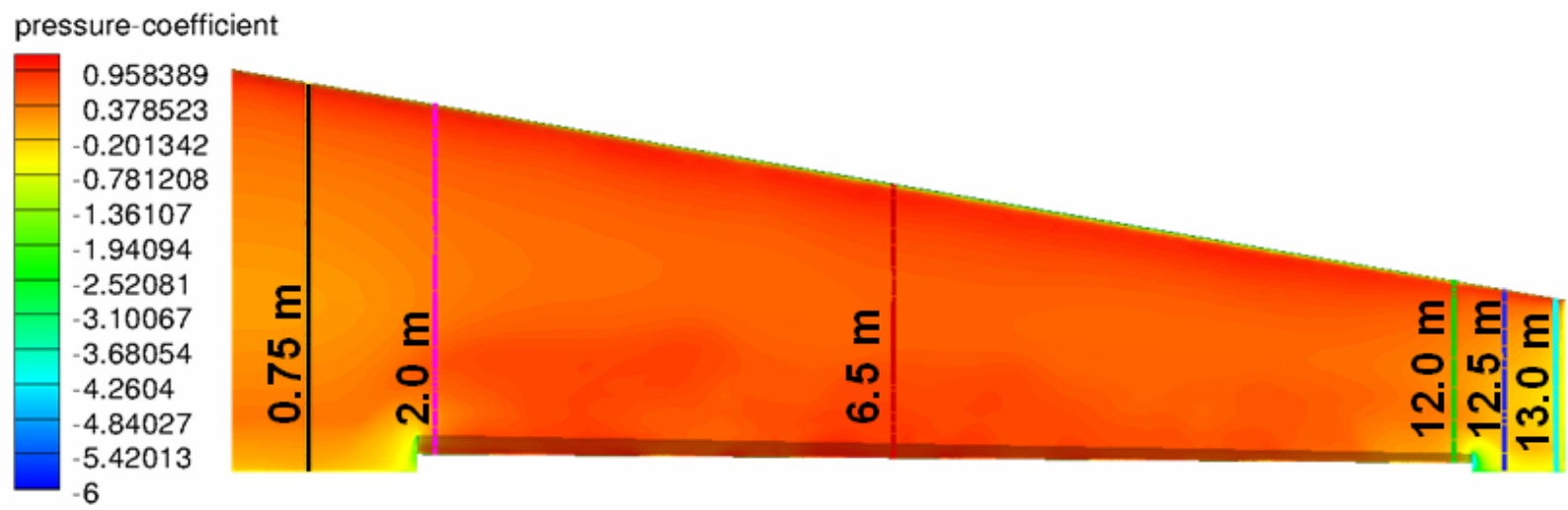

Figure 8-20: CP Cross Section Locations 


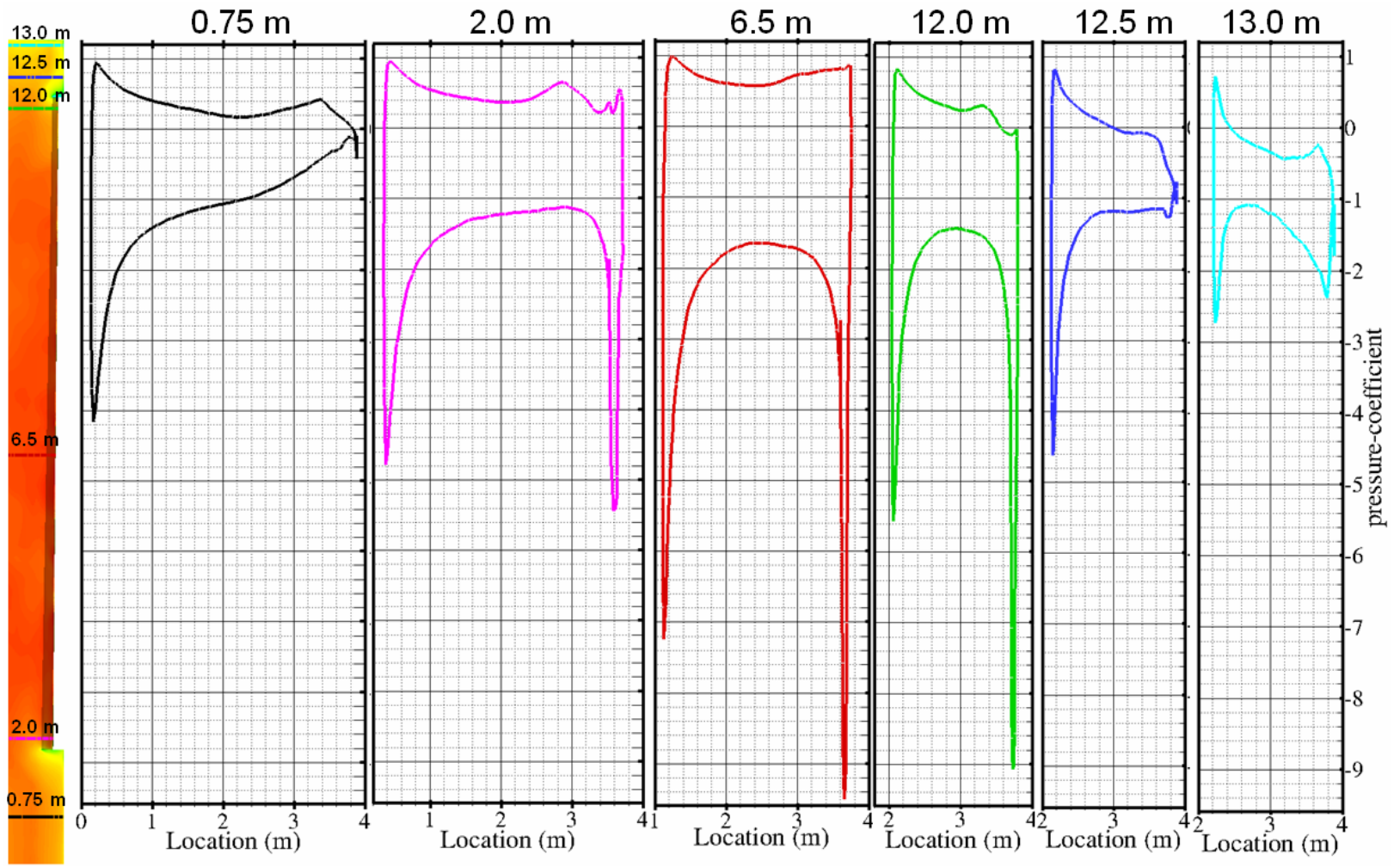

Figure 8-21: $\mathrm{C}_{\mathbf{P}}$ Sections

The location, as depicted by the various $\mathrm{X}$-axes, was measured from the leading edge of the root chord while the spanwise slices of $0.75 \mathrm{~m} ., 2.0 \mathrm{~m} ., 6.5 \mathrm{~m} ., 12.0 \mathrm{~m} ., 12.5 \mathrm{~m}$. , and $13.0 \mathrm{~m}$. were simply measured from the root chord. From the figure it can be seen that the center of the center of the wing with the $\mathrm{CC}$ flap clearly produces the most amount of lift with the minimum $\mathrm{C}_{\mathrm{P}}$ dropping below negative nine. It is also interesting to note that the outboard junction $(12 \mathrm{~m})$ of the $\mathrm{CC}$ flap has a much lower $\mathrm{C}_{\mathrm{P}}$ values than the inward junction $(2 \mathrm{~m})$. From these results it can be inferred that the spanwise lift distribution for this $\mathrm{CCW}$ is skewed towards the outboard section of the wing. Also, as with many high lift devices, the large pitch-down moment from the CCW can clearly be seen from the large negative $C_{P} s$ at the trailing edge which for this case generated a $C_{M}$ of 0.6294 . The large moments along with the outwardly skewed lift distribution will require significant 
consideration in the design of a STOL transport, specifically in the areas of structural support for the CCW and tail as well as the overall tail sizing and controllability of the aircraft.

As was shown before, an additional example of the flowfield characteristics for $\mathrm{CCW}$ is in the wake. The final case for which the wake was analyzed occurred at a Mach number of 0.15 , an angle of attack of zero degrees, a mass flow rate of $15 \mathrm{~kg} / \mathrm{s}$, and a flap deflection of $60^{\circ}$.

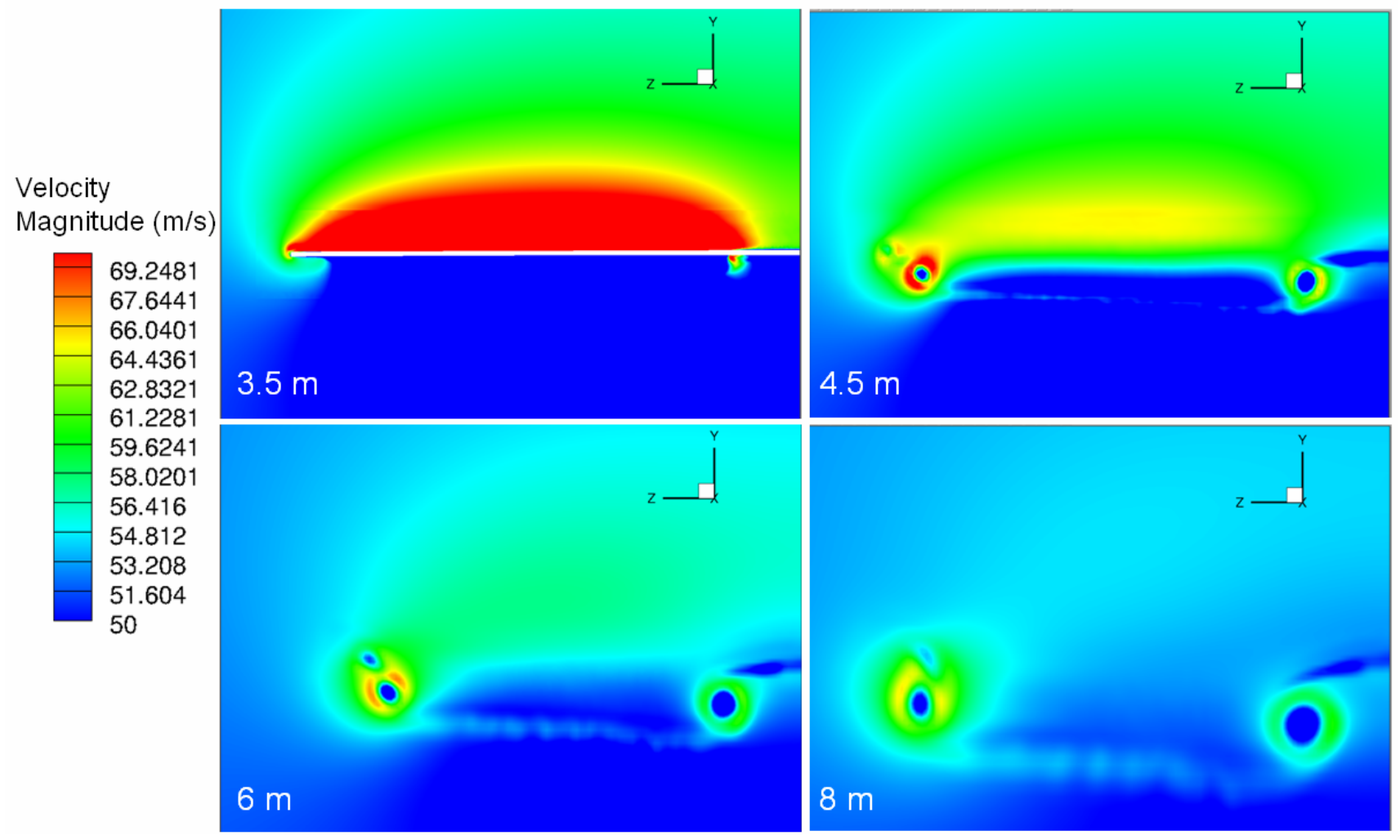

Figure 8-22: Wake Profile with Trailing Vortices

As before, the figure shows the velocity magnitude contour slices at several locations through the flowfield. The slices of $3.5 \mathrm{~m} ., 4.5 \mathrm{~m} ., 6 \mathrm{~m}$., and $8 \mathrm{~m}$. were measured from the leading edge of root chord. In the $3.5 \mathrm{~m}$. slice, the entire half wing can be seen from the back as well as the high speed flow over the top of the wing and the beginnings of the 
trailing vortices. The other three slices illustrate how the three major vortices from the two edges of the CC flap and the wing tip progress downstream of the wing. From the last three slices it is clear that the vortex which emits from the outboard junction the $\mathrm{CC}$ flap has a much higher intensity than the inward vortex. This agrees with the previous analysis of Figure 8-21 which shows the $C_{P}$ in the outboard region to be much more negative than that of the inboard.

With the high quality Icem grids producing solutions that accurately resolved the boundary layer and flowfield turbulence, the next step was to incorporate this data into the final aerodynamic metamodel. However, before the model could be used in the BFL simulator, it was important to ensure that there were enough CFD cases defining the model. As with the previous models, a point by point error analysis was performed in order to analyze the new metamodel. The results of one of the error analysis techniques can be seen in Figure 8-23.

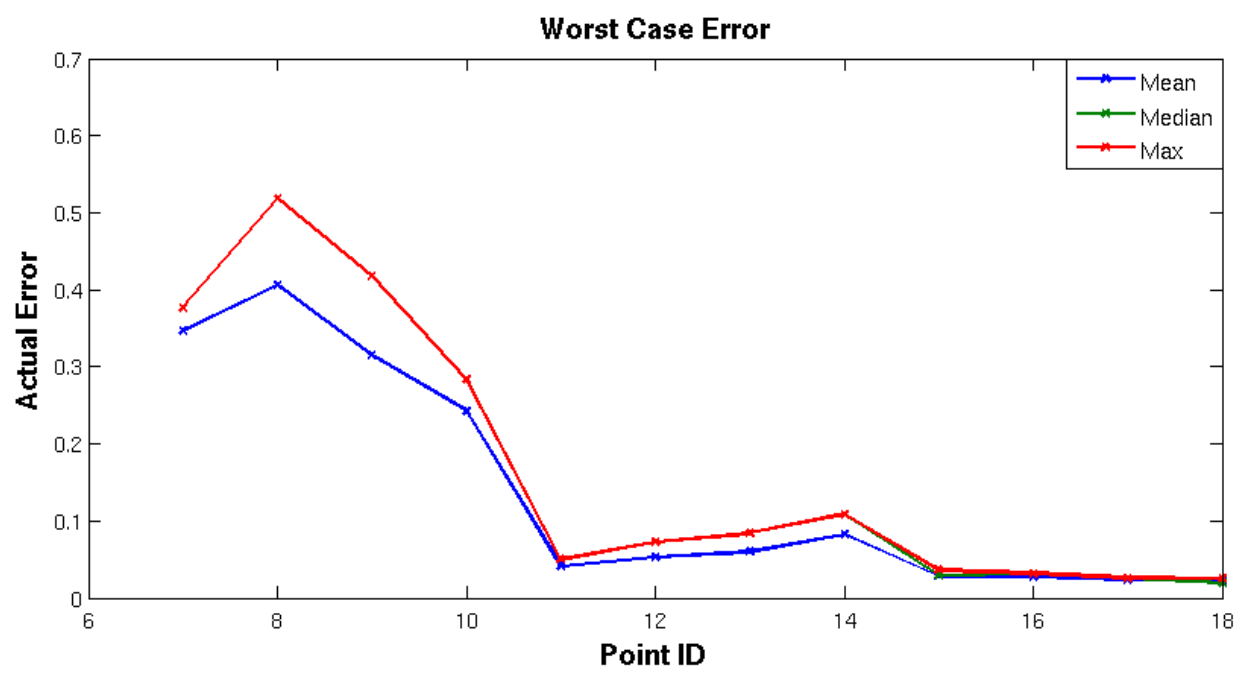

Figure 8-23: Error Analysis for Final Metamodel

For the final metamodel, the number of dimensions was reduced from four to three, eliminating flap deflection. With the design space reduced by one dimension, the number 
of CFD points required to fully define the design space significantly decreased. As can be seen from Figure 8-23, only eight points were required before the error began to show a convergence trend. After eighteen cases, it was determined that the error of the model had reached steady state and therefore the 3D model had been fully defined. For more detail on the error analysis, including additional error methods, please consult Mr. Turner's thesis ${ }^{34}$. A slice of the final 3D metamodel can be seen in Figure 8-24 set at a Mach number of 0.205 , angle of attack of $2.83^{\circ}$, and a mass flow rate of $8.12 \mathrm{~kg} / \mathrm{s}$.

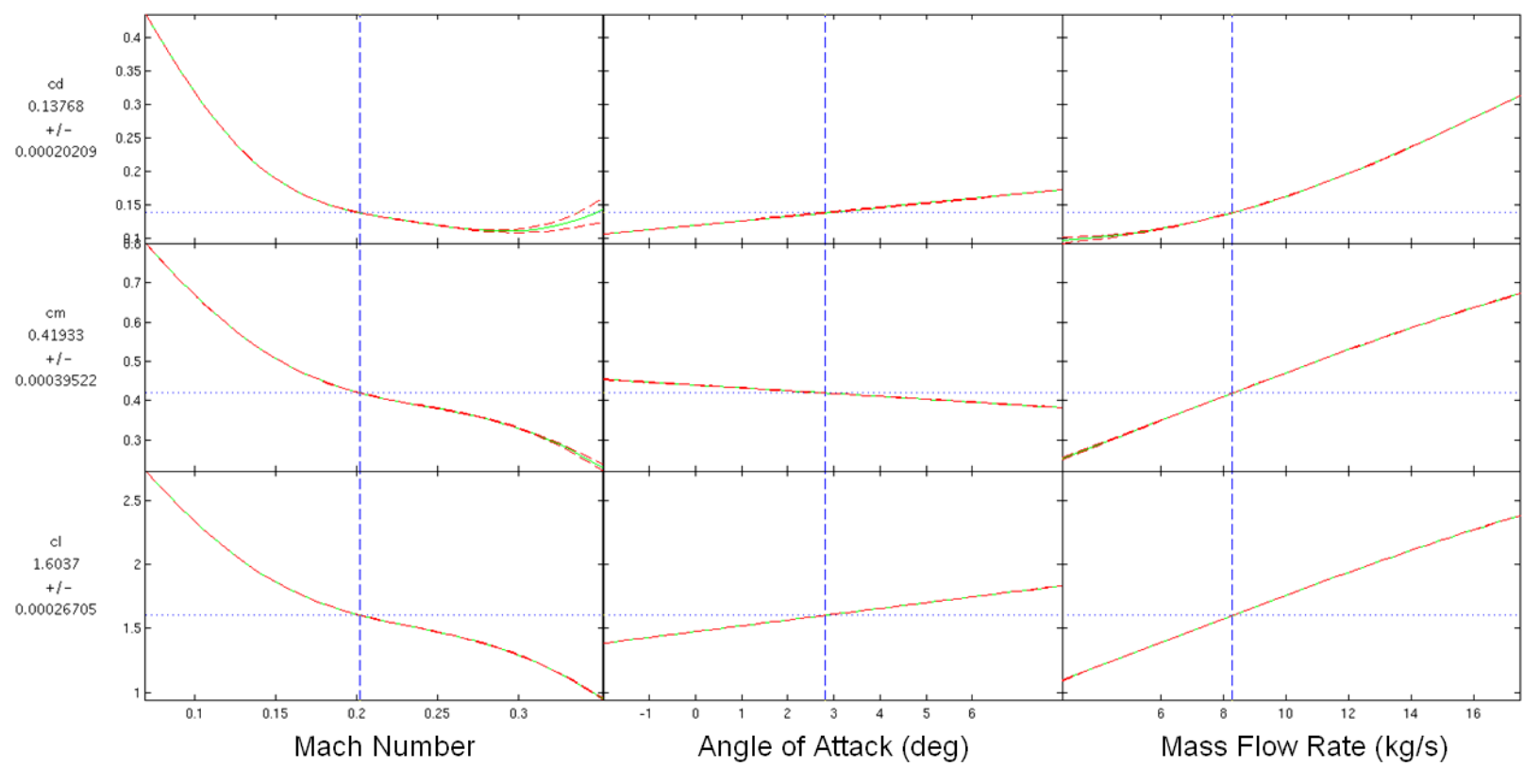

Figure 8-24: Final Metamodel

After examining the results of this metamodel, it was found that some of the results did not match commonly known trends. For example, a traditional drag polar depicts a nonlinear relationship between $\mathrm{C}_{\mathrm{D}}$ and angle of attack. Another unexpected result was the lack of stall in the $\mathrm{C}_{\mathrm{L}}$ vs. alpha relationship. Many studies of CCW have shown that the enormous amount of lift generated by the supercirculation can cause leading edge separation to occur at even moderate angles of attack. However, the metamodel showed no hint of decreased performance up to almost $8^{\circ}$. 


\subsection{Metamodel Verification}

To verify the relationships between $C_{L}$ and $C_{D}$ versus angle of attack, several more $3 \mathrm{D}$ cases were run holding the Mach number and mass flow rate constant and varying the angle of attack. Specifically, the Mach number was set to 0.181 and the mass flow rate was set at $15.4 \mathrm{~kg} / \mathrm{s}$ while the angle of attack was varied from $0-15^{\circ}$.

The first trend which will be analyzed is the relationship between $\mathrm{C}_{\mathrm{L}}$ and angle of attack. Traditionally there is a linear relationship which eventually changes to a nonlinear after stall. The metamodel predicted simply a linear trend between lift and angle of attack with no hint of stall. After running five new CFD cases, the relationship between $C_{L}$ and angle of attack could be verified and can be seen in Figure 8-25.

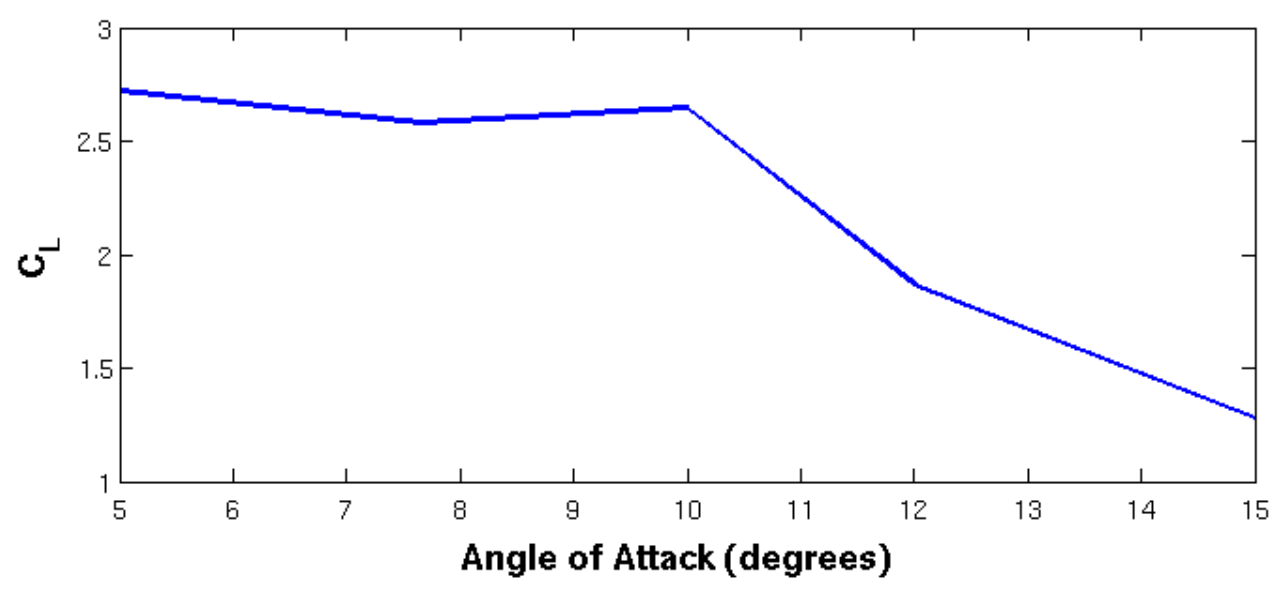

Figure 8-25: $C_{L}$ as a Function of Angle of Attack

The first feature from this figure which stands out is the stall region which occurs after $10^{\circ}$ angle of attack. It is also clear that the results before stall are mostly linear with very little increase in $C_{L}$ at higher angles of attack. These results agree with the metamodel results which also show the linear relationship. The nonlinear section does show up in the metamodel because stall does not occur until after $10^{\circ}$ while the metamodel included 
angles of attack between $-2^{\circ}$ and $8^{\circ}$. If the stall region is a feature which one would want in the metamodel, the design space should be increased to beyond $10^{\circ}$. However, from these results and from the metamodel it is clear that the increase in lift at higher angles of attack is minimal so that from a performance standpoint it is unnecessary to rotate the aircraft anywhere near the stall region. The Mach number, mass flow rate, and flap deflection have a much larger impact on $\mathrm{C}_{\mathrm{L}}$ for a $\mathrm{CCW}$ aircraft than angle of attack.

The next verification to take place was for the relationship between drag and angle of attack. Traditionally, drag increases non-linearly with angle of attack. However, the metamodel showed nearly a linear relationship. The results from the five additional CFD cases which show the relationship between drag and angle of attack can be seen in Figure 8-26.

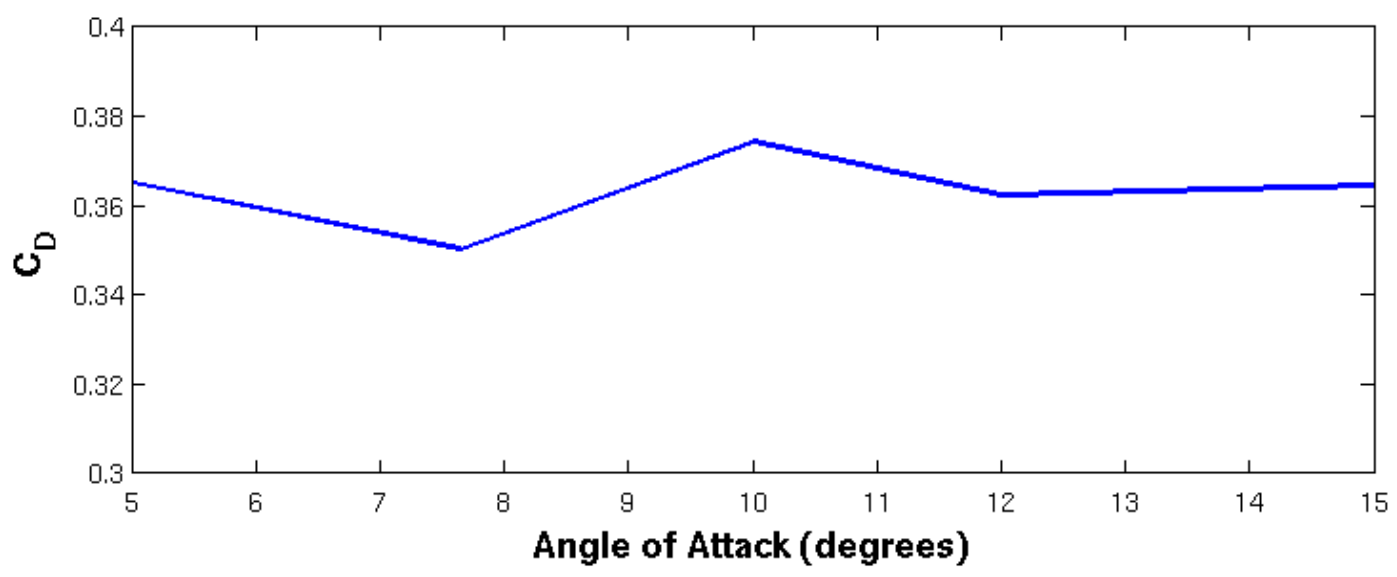

Figure 8-26: $C_{D}$ as a Function of Angle of Attack

A couple of conclusions can be made from these results. First, the non-linear relationship which was not present in the metamodel is also not present here. The next interesting point comes from observing the behavior of $C_{D}$ in the stall region after $10^{\circ}$. Normally, the drag will increase after stall whereas these results show a small decrease in CD. This could in part be explained by the huge amount of induced drag which was no longer 
present after the wing stalled. The decrease in induced drag counteracted the increase in viscous drag and caused the results which were shown. To verify this explanation, the induced drag for each of the cases was calculated using Eq(20), assuming an efficiency factor of 0.8 and with an aspect ratio of 9.5 .

$$
C_{D_{i}}=\frac{C_{L}^{2}}{\pi e A R}
$$

The viscous drag was calculated simply by subtracting the estimated induced drag from the total drag obtained from the CFD. The relationship of the calculated viscous drag with angle of attack can be seen in Figure 8-27.

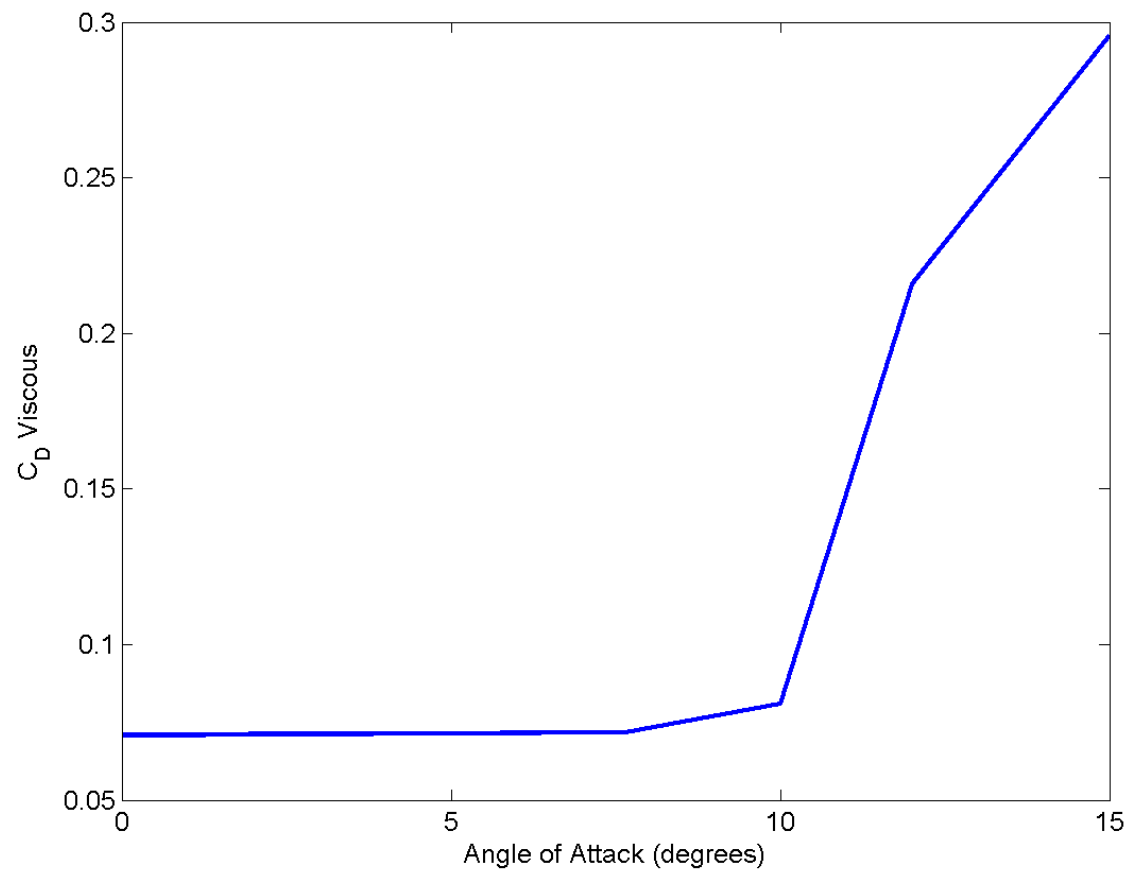

Figure 8-27: Non-Linear Relationship of $C_{D}$ and Angle of Attack

From this figure it is clear that the non-linear relationship was being captured in the CFD but was completely obscured by the large amounts of induced drag. These results show 
that the amount of induced drag present before separation was roughly equal to the amount of viscous drag after separation. This explains why the metamodel correctly showed the linear relationship between $\mathrm{C}_{\mathrm{D}}$ and angle of attack

\subsection{Design Space Examination}

With the final metamodel in place, some additional analysis was performed of the BFL design space. The original 4D design space of Mach number, angle of attack, mass flow rate, and flap deflection was specified in such a way so that the BFL could determined by integrating the equations of motion throughout the takeoff. However, with the final model in place, it was of interest to examine a typical execution of the BFL simulation in order to find the actual values of $\mathrm{C}_{\mathrm{L}}, \mathrm{C}_{\mu}$, and Mach number which the aircraft experiences during the BFL and how much the metamodel was required to extrapolate.

To examine the design space, the BFL simulator was run with the same STOL transport as before with a constant flap deflection of $60^{\circ}$ and a constant mass flow rate of $15 \mathrm{~kg} / \mathrm{s}$. The first results to be discussed will be $\mathrm{C}_{\mathrm{L}}$ as it varied with time and velocity as shown in Figure 8-28. Since this analysis is for a balanced field length, two curves are shown: one for the aborted takeoff and one for the continued takeoff. For this case, the critical point at which the engine was said to fail occurred at 8.3 seconds after which the reaction time of three seconds occurred, and finally both the braking and takeoff sequences were simulated. 

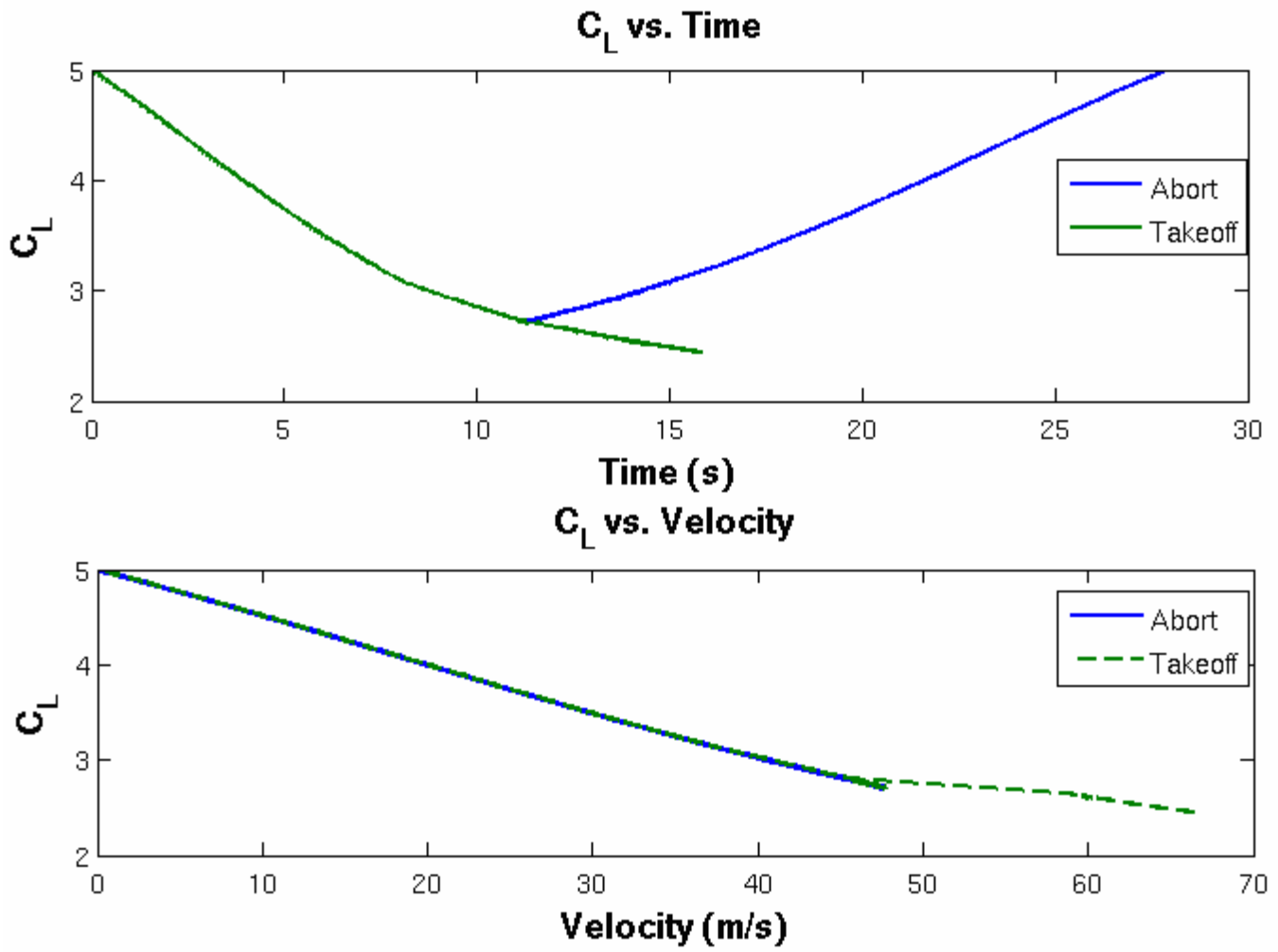

Figure 8-28: $C_{L}$ Variations during BFL

From the plots, we can see that the $\mathrm{C}_{\mathrm{L}}$ begins and ends at about 5 as the aircraft begins moving and as it comes to a stop. The higher $\mathrm{C}_{\mathrm{L}}$ values occur at the lower Mach numbers where $\mathrm{CCW}$ is known to create more lift. For this case with mass flow rate of $15 \mathrm{~kg} / \mathrm{s}$, and $\mathrm{C}_{\mathrm{L}}$ values over about 3.8 are being extrapolated due to limited solutions at the lower Mach numbers. As a result, the metamodel was forced to extrapolate for about 10 seconds of the aborted takeoff and 5 for the continued takeoff. However, this problem could not simply be fixed by running more CFD cases since stability issues prevented cases from converging at Mach numbers less than 0.06. The extrapolation will have to continue until a solution to the instability problem can be found.

The next examination of the design space was of the Mach number versus time during the same BFL, the results of which are shown in Figure 8-29. 


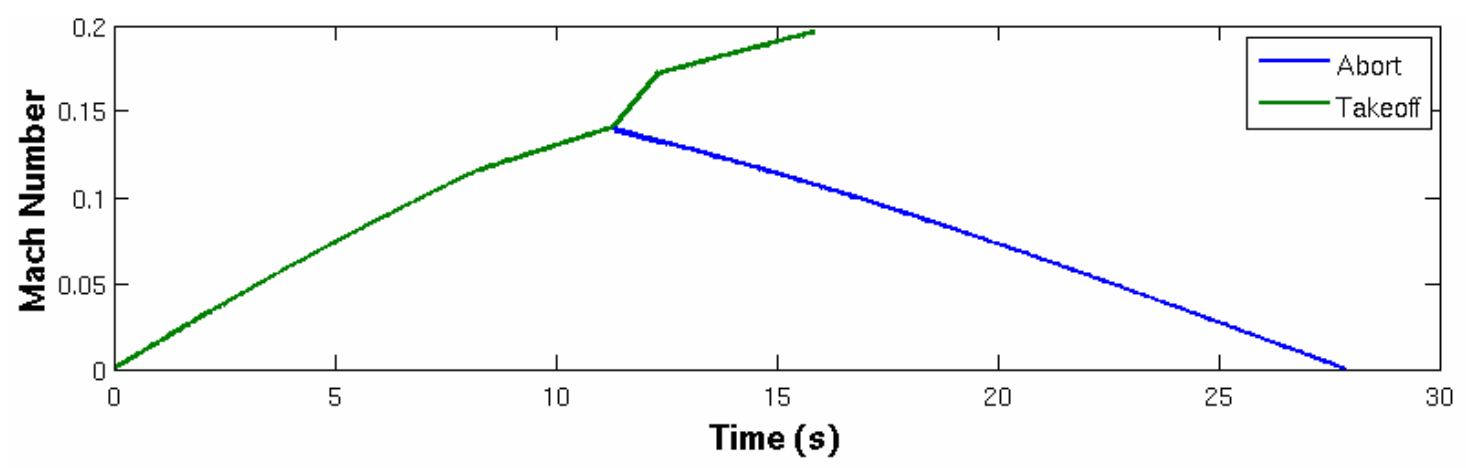

Figure 8-29: Mach Number During a BFL

From this plot it can be seen that for this case the Mach number does not exceed 0.2. The design space included data that went all the way to Mach 0.35 in order to capture every type of BFL. However, from this plot it is clear that Mach 0.35 is much higher then necessary. If the aerodynamic model was to be created again, the Mach number space could be reduced to a little over Mach 0.2. Again, since the CFD cases were limited on the lower end to Mach numbers of 0.06, it can be seen that the metamodel was forced to extrapolate for about ten seconds of the aborted takeoff and less than five for the continued takeoff.

The final examination of the design space showed how the blowing coefficient $\mathrm{C}_{\mu}$ varied versus time and velocity during the same BFL. The results of this analysis can be seen in Figure 8-30. 

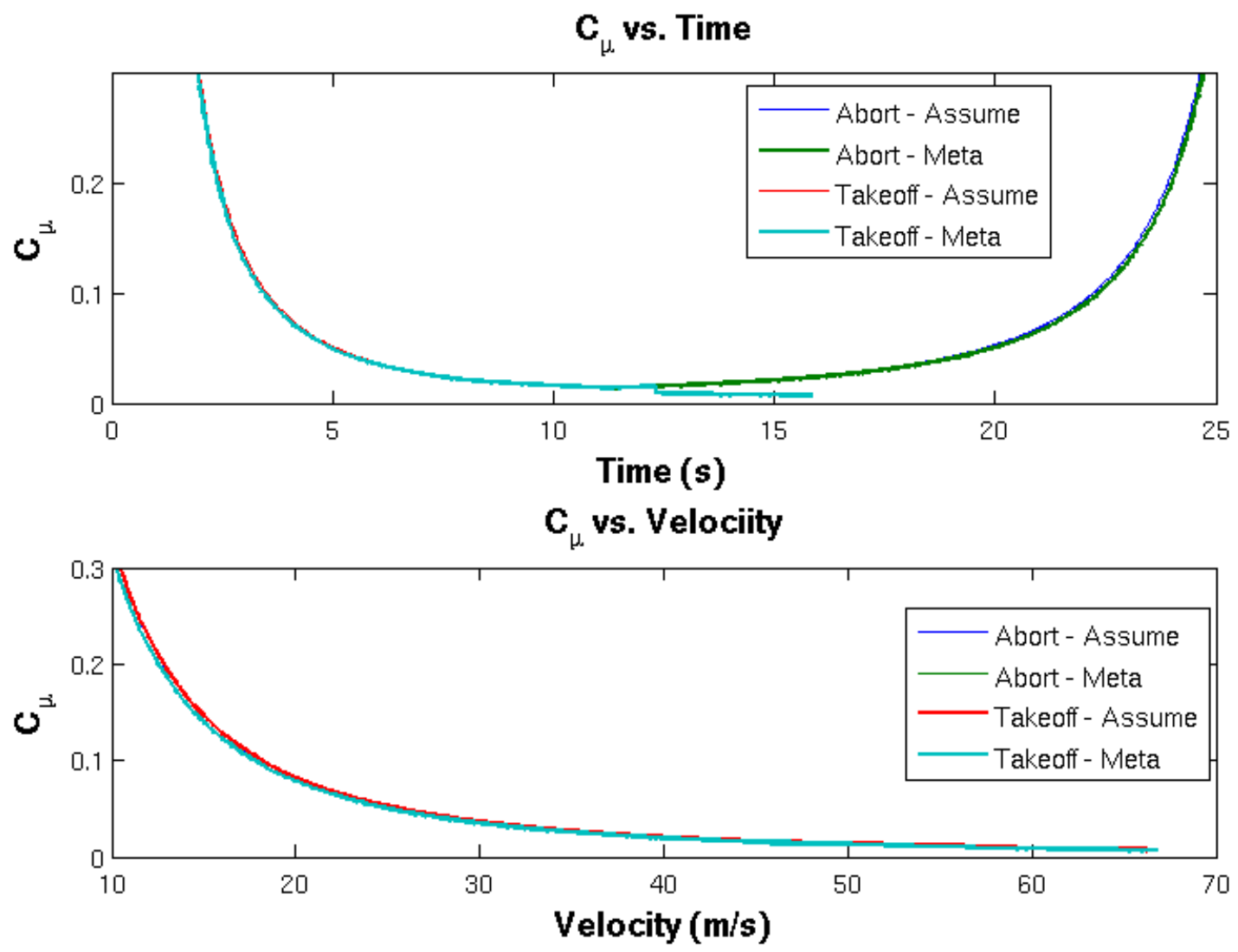

Figure 8-30: Cmu Variations During a BFL

The $\mathrm{C}_{\mu}$ was calculated using two different methods for these plots, the solutions of which agreed really well. The first method, known as the assumed method, used the assumption that the density at the ejection slot was the same as the freestream to calculate an unknown jet velocity, the equation for which is given as $\mathrm{Eq}(21)$.

$$
C_{\mu}=\frac{\dot{m} V_{j e t}}{q_{\infty} S}=\frac{\dot{m^{2}}}{q_{\infty} S A_{j e t} \rho_{j e t}}
$$

The other method, termed "meta", used the regression relationship as given in Eq(18) and repeated here again for convenience as $\mathrm{Eq}(22)$.

$$
V_{j e t}=-.1791 \dot{m^{2}}+20.3253 \dot{m}-2.6022
$$


Again, the two methods agreed very well for this test case. However, the results would have been very different if the temperature at the jet slot was not set to the ambient temperature, as were all of the cases throughout this research. By changing the jet temperature, the density at the ejection slot would vary according to the perfect gas law and would therefore be very different from the freestream density. Also, the regression from $\mathrm{Eq}(22)$ would be invalid since it was also built off data with the jet temperature set to the ambient temperature.

However, since both models worked very well for the case at hand, a couple of conclusions can be made from Figure 8-30. First, since $C_{\mu}$ is a function of the freestream dynamic pressure, the figure shows how $\mathrm{C}_{\mu}$ dramatically increases at the lower velocities. For this research, the design space was discritized using mass flow rate rather than $\mathrm{C}_{\mu}$ for reasons discussed in section 4 . If $C_{\mu}$ was used in its place, the very high $C_{\mu}$ values would either need to be achieved by specifying an unrealistic high mass flow rates or by lowering the freestream Mach number. Again, since there are instability constraints at the lower Mach numbers, achieving the high $\mathrm{C}_{\mu}$ solutions becomes difficult.

As a result of the extrapolation in the regions of high $\mathrm{C}_{\mu}$ values, the lift and consequently the drag increase significantly in those regions. Although both $C_{L}$ and $C_{D}$ were being overestimated at the lower Mach numbers, the increased amount of drag only extended the BFL rather than error on the short side. Until a better method for obtaining CFD solutions at lower Mach numbers is found, the extrapolation used in those regions will need to be built into the BFL distance as a safety factor. 


\subsection{Final BFL Performance}

Finally, with the model verified, the BFL for the STOL transport could be simulated for a third and final time. Although the preliminary and interim models included errors due to grid dependence, $\mathrm{y}^{+}$resolution, and thrust simplifications, these errors were all corrected for the final model, the results of which can be seen in Figure 8-31. Some of the previous discrepancies can easily be seen in the figure. The preliminary results underestimated the amount of lift and drag by over $7 \%$ which added over 300 feet to the minimum BFL and the interim results had over a $9 \%$ underestimate in the total drag which predicted the minimum BFL over 130 feet short. The final model clearly shows that the $230,000 \mathrm{lb}$. STOL transport using CC can achieve BFLs between $2,884 \mathrm{ft}$ to $2,073 \mathrm{ft}$ depending on how much mass flow is available.

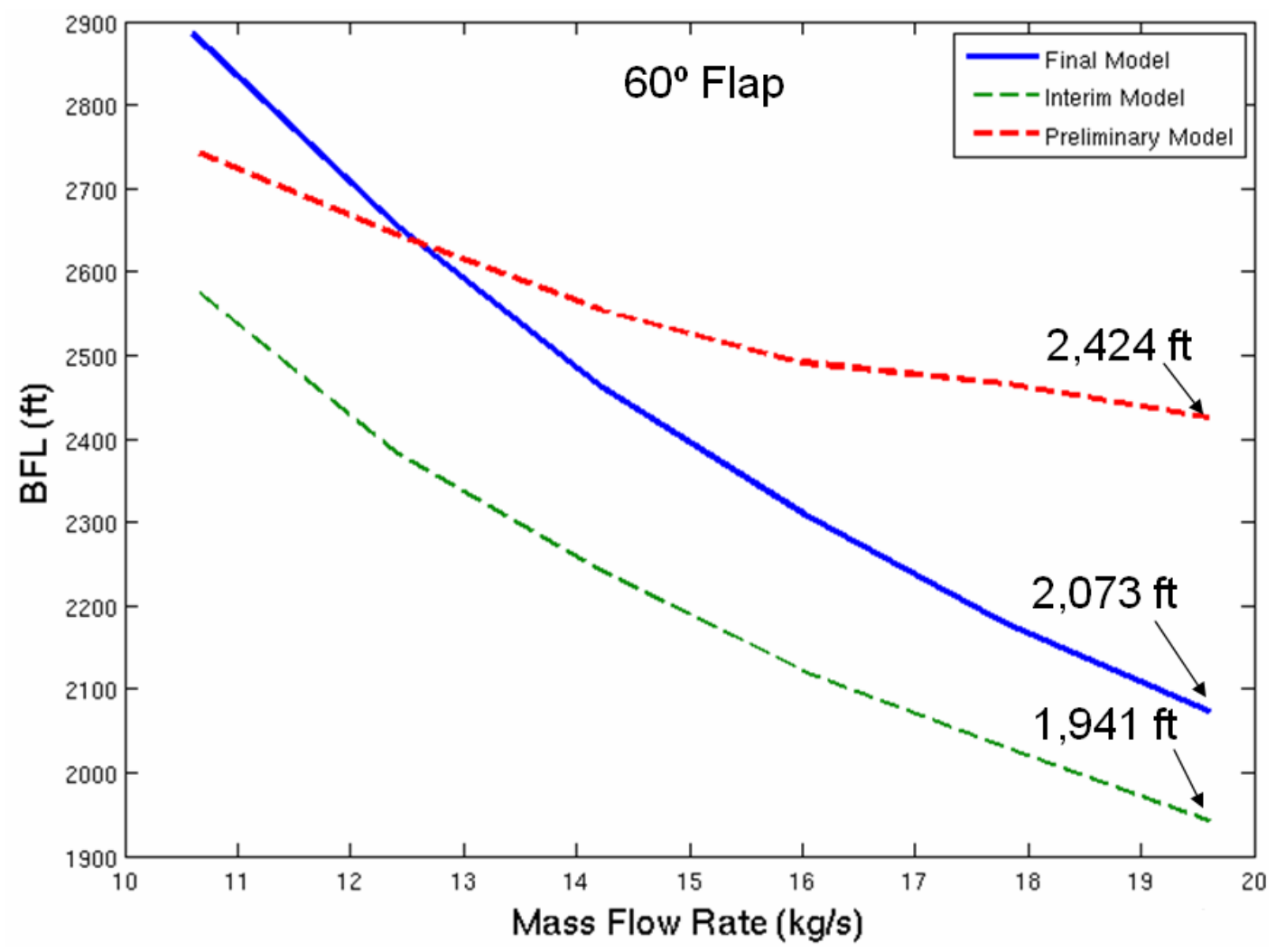

Figure 8-31: BFL (ft) vs. Mass Flow Rate (kg/s) for all Three Models with $6^{\circ}$ Flap 


\section{Conclusions}

Although calculating a balanced field length is an essential component in designing a STOL aircraft, no accurate methods exist for powered lift aircraft. Almost all traditional techniques rely totally on historical data based off traditional aircraft. Circulation control, like USB and EBF, is a high lift device which can enable a large aircraft to achieve takeoff and landings on runways with distances less than $2500 \mathrm{ft}$.

Three different metamodels were created using a Monte Carlo approach with Gaussian Processes, each with increasing fidelity. The first two models were based off of aerodynamic data obtained with Fluent from 3D grids generated using Gambit. The first model allowed for the basic framework to be laid for populating the four-dimensional design space with CFD points. The second model was also created using Gambit, but with a better $30 \%$ volume growth rate. Although the $30 \%$ growth rate performed reasonably well with respect to a couple of lower growth rates also performed in Gambit, the turbulence generated from the boundary layer was not able to be resolved well with any of them since the grids did not contain boundary layer cells to reduce the surface $\mathrm{y}^{+}$ values. This problem was finally remedied with the use of the final metamodel which was based off of aerodynamic data generated from grids with Icem. For each of these metamodels, the gridding and solving methodologies and automations were detailed and samples of some of the automation scripts were provided in the appendices.

The flow features of $\mathrm{CC}$ were examined throughout each of the three models including the effects of supercirculation on the surrounding flowfield and the effect the turbulence model has on predicting separation from the circulation control flap. It was found that the one-equation Spalart-Allmaras model predicted the freestream separation 
from the high speed jet flow at a similar point and with similar values for $C_{L}$ and $C_{D}$ as the k- $\varepsilon$ and k- $\omega$ SST two-equation turbulence models.

Throughout this study, the effects of obtaining reasonable surface $\mathrm{y}^{+}$values were repeatedly tested. The first models attempted to model turbulence without any boundary layer cells and rely on the base surface meshes and $\mathrm{y}^{+}$adaption within Fluent. Although this technique allowed for a lower initial cell count, the adaptions ended up adding a significant number of cells and never completely lowered the surface $\mathrm{y}^{+}$to acceptable levels. With these grids, there was little effect to the $C_{L}$ and surprisingly little effect on $\mathrm{C}_{\mathrm{D}}$. The boundary layer was finally resolved with the final metamodel which incorporated boundary layer cells on all of the wing surfaces. However, three iterations of generating grids with modified initial cell heights were required before nearly all of the surface cells had $\mathrm{y}^{+}$values outside of the buffer region which then provided good results for the viscous drag.

The BFL results for each of the metamodels were provided by inserting the models into a simulation code developed for this process. The equations of motion were integrated for each of the major segments of takeoff as well as during braking for the engine out case. The definition of the rotation velocity was derived to be a function of lift to weight rather than being based on $C_{\mathrm{Lmax}}$ which is a difficult parameter to define for an aircraft using circulation control. The result for the first two metamodels included performance plots of how the BFL varied with respect to dual radius flap deflection and mass flow rate. The first metamodel also was used to explore how a BFL could be optimized by varying the flap or mass flow rate during the ground roll which resulted in almost a 9\% decrease in BFL. The contours of BFL vs. flap deflection at various mass 
flow rates presented an optimal flap deflection between $60-64^{\circ}$ which was then used in the final model. From the results of the three models using the STOL transport configuration, the shortest BFL varied from 2,424 ft. for the preliminary model, to 1,941 $\mathrm{ft}$. for the interim, and finally to $2,073 \mathrm{ft}$. with the final model.

As a result of this research, a tool is now available for use which can be used in early design of a STOL aircraft utilizing circulation control. By generating high fidelity CFD aerodynamic data and creating Gaussian Process metamodels, the BFL performance of a STOL aircraft utilizing circulation control can now be accurately predicted for many different takeoff configurations. 


\section{Future Work}

This research could be taken in a number of different directions which could add both to its capabilities and accuracy. Any of the simplifications which were used in this research can be replaced with the real world equivalent in order to add more accuracy to the results. For example, a plenum chamber could be modeled instead of simply applying a mass flow inlet boundary condition to the ejection slot face. Along those same lines, some kind of inlet can be included as the source for the blowing which could either be one of the aircrafts primary engines or a dedicated gas generator within the wing with its accompanying inlet. An additional simplification that was made in this research was that the lift and drag for the wing was taken to be the total lift and drag for the aircraft. This can be improved by including half a fuselage as well as a tail. Along with the tail comes the aspect of controllability. As with all high lift devices, CC produces an enormous pitching moment which needs to be trimmed with the tail. Additional research could be performed which couples in the ability to size the tail and or the use of $\mathrm{CC}$ on the tail. Finally, the addition of ground effect to the metamodel would add another level of accuracy to the simulation since in the real world the ground will be present and either add to or detract from the CCW performance. The framework has been laid for any of this additional research to be performed and included into a metamodel and within the BFL simulation. 


\section{References}

\footnotetext{
${ }^{1}$ U.S. Airforce Central. www.centaf.af.mil/photos.

${ }^{2}$ Advanced Medium STOL Transport (AMST): YC-14 and YC-15 http://www.globalsecurity.org/military/systems/aircraft/amst-pics.htm. April 27, 2005

${ }^{3}$ Englar, Robert J, Hemmerly et al. "Design of the Circulation Control Wing STOL Demonstrator Aircraft." Journal of Aircraft, Vol. 18 No. 1. January 1981. AIAA79-1842R

${ }^{4}$ Mayfield, Jerry. "Circulation Control Demonstrates Greater Lift." Aviation Week and Space Technology, March 19, 1979.
}

5 Anderson, John D. Aircraft Performance and Design. McGraw-Hill Science Engineering, New York. Ch 6. 1998.

${ }^{6}$ Federal Aviation Administration. Regulatory and Guidance Library.. Washington D.C. 2007. Part 25: 105, 107, 109, 111, 113, 121. http://rgl.faa.gov/Regulatory_and_Guidance_Library

${ }^{7}$ Torenbeek, Egbert. Synthesis of Subsonic Airplane Design. Delft University Press, Delft, The Netherlands. Ch5 and App. K 1982.

${ }^{8}$ Englar, Robert J. Overview of Circulation Control Pneumatic Aerodynamics: Blown Force and Moment Augmentation and Modification as Applied Primarily to Fixed-Wing Aircraft. NASA/ONR Circulation Control Workshop, March 16-17, 2004, NASA/CP-2005-213509.

${ }^{9}$ Englar, Robert J., and Gregory G. Huson. "Development of Advanced Circulation Control Wing HighLift Airfoils.” Journal of Aircraft, Vol. 21, No. 7, (1984): 476-483.

${ }^{10}$ White, Frank M. Viscous Fluid Flow, Third Edition. McGraw-Hill Co. Inc. New York, New York. 2006

${ }^{11}$ Tannehill, John C., Anderson, Dale A., Pletcher, Richard H. Computational Fluid Mechanics and Heat Transfer, Second Edition. Taylor and Francis, 1997. pp. 301-305

${ }^{12}$ Hoffmann, Klaus A., Chiange, Steve T. Computational Fluid Dynamics, Volume III, Fourth Edition. Engineering Education System. Wichita, Kansas. 2000

${ }^{13}$ de la Montanya, Julianna Belle. Circulation Control and Its Application to ESTOL Vehicles, Master's Thesis, California Polytechnic State University, San Luis Obispo, CA. June 2006.

${ }^{14}$ Abramson, J., and E.O. Rogers. High-Speed Characteristics of Circulation Control Airfoils. AIAA-830265.

15 Jones, Gregory S., Robert J. Englar. Advances in Pneumatic-Controlled High-Lift Systems through Pulsed Blowing. AIAA Paper 2003-3411.

${ }^{16}$ Lui, Yi et al. Computational Evaluation of the Steady and Pulsed Jet Effects on the Performance of a Circulation Control Wing Section. 2004 NASA/ONR Circulation Control Workshop, March 16-17, 2004, NASA/CP-2005-213509, pp.295-336

${ }^{17}$ McGowan, Gregory and Gopalarathnam, Ashok. Computational Study of a Circulation Control Airfoil Using FLUENT. AIAA Paper. North Carolina State University, North Carolina. 2005 
${ }^{18}$ Jones, G. S., Viken, S. A., Washburn, A. E., Jenkins, L. N., and Cagle, C. M., An active Flow Circulation Controlled Flap Concept for General Aviation Aircraft Applications. AIAA Paper 2002-3157, 2002

${ }^{19}$ McGowan, Gregory, Gopalarathnam, Ashok, and Xiao, Xudong. Role of Turbulence Modeling in Flow Prediction of Circulation Control Airfoils. AIAA Paper. 2005.

${ }^{20}$ Abramson, J., and Rogers., E. O., High-Speed Characteristics of Circulation Control Airfoils. NASA/ONR Circulation Control Workshop, March 2004

${ }^{21}$ McGowan, Gregory, Rumsey, Christopher, Swanson, R. Charles, Hassan, Hassan. A Three-Dimensional Computational Study of a Circulation Control Wing. AIAA Paper 2006-3677. North Carolina State University, June 2006.

${ }^{22}$ Hall, Dave et. al. Summer 2004 CalPoly/NASA ESTOL Work Presented to NASA/Ames ESTOL Vehicle Systems Sector. PowerPoint Presentation. August 27, 2004.

${ }^{23}$ Liu, Yi, Sankar, Lakshmi N., Englar, Robert J., Ahuja, Krishan K. Numerical Solutions of the Steady and Unsteady Aerodynamic Characteristics of a Circulation Control Wing. GTRI Report A5928/2003-1 App. B. Georgia Tech Research Institute, GA 2003.

${ }^{24}$ Fluent 6.3 Users Guide Fluent Inc. www.fluentusers.com/fluent/doc/ori/html/ug/main_pre.htm

${ }^{25}$ Percey, Bobbitt J., Eagle Aeronautics, Margason, Richard. Analysis of the Take-off and Landing of Powered Lift Aircraft. AIAA, 2007

${ }^{26}$ Ball, Tyler, Turner, Scott, Marshall, David D. Short Takeoff Performance Using Circulation Control. AIAA 2008-174

${ }^{27}$ Rasmussen, Carl Edward, Williams, Christopher K.I. Gaussian Processes for Machine Learning. The MIT Press, Cambridge Massachusetts, 2006

${ }^{28}$ McDonald, Robert A. Error Propagation and Metamodeling for a Fidelity Tradeoff Capability in Complex System Design. Ph.D Thesis, Georgia Institute of Technology, GA, 2006

${ }^{29}$ Rasmussen, Carl Edward, Williams, Christopher K.I. Gaussian Processes for Machine Learning. The MIT Press, Cambridge Massachusetts, 2006

${ }^{30}$ Lynn, Sean. Summary Report for an Undergraduate Research Project to Develop Programs for Aircraft Takeoff Analysis in the Preliminary Design Phase, Undergraduate Research Project, Virginia Polytechnic Institute and State University. Blacksburg, Va, May 11, 1994.

31 Hank, C.R., et al, "The Simulation of a Jumbo Jet Transport Aircraft. Volume 2: Modeling Data," NASA CR 114494, also N73-10027 Sept. 1970.

32 Powers, S. A., "Critical Field Length Calculations for Preliminary Design,” Journal of Aircraft, Vol. 18, No. 2, 1981

33 Krenkel, A.R., Salzman, A., "Takeoff Performance of Jet-Propelled Conventional and Vectored-Thrust STOL Aircraft," Journal of Aircraft, Vol. 5, No. 5, 1968

34 Turner, Scott. Applied Metamodeling for Aerospace Applications. Master's Thesis, California Polytechnic State University, San Luis Obispo, CA, May 2008. 


\section{Appendix A - Mesh-Sender Script}

The purpose of this appendix is to briefly explain the major blocks of code in the Mesh-Sender script. It was written entirely in Bash shell scripting language and can be executed simply by typing "./Mesh-Sender" into the command line with the script in the working directory.

\section{Lines 5-32: Flap Deflection}

This while loop queries the user for the flap deflection. Since each flap deflection required a different grid, this question helped to narrow which grid was going to be used. Lines 14-18 make sure there is a leading zero in front of the flap deflection in case it is less than ten degress. Lines $20-30$ check to see if the requested flap deflection grid is available.

\section{Lines 36-52: Blowing or No Blowing}

For several reasons, it was necessary to run Fluent cases with the circulation control turned off. Within Fluent, the software was unable to handle a mass flow inlet with zero mass flow. To solve this problem, an additional grid was created for each flap deflection that changed the boundary condition of the jet slot from mass flow inlet to a wall. The decision as to whether or not there is blowing altered what template file was used for the Fluent case and whether or not a new grid would be created by Gambit. This will be discussed for lines 274-335.

\section{Lines 55-242: Ground Effect}

This significant portion of the script asked whether or not ground effect was going to be used, checked to see if the grid was available, and if not, generated a new grid. 
Since there were so many combinations of ground height and angle of attack, each of which required a new grid, many ground effect grids were created from this script.

\section{Lines 62-63: Mesh Folder and Template}

The 3-D meshes were separated into two folders: Meshes and G-Meshes which held all the currently available normal meshes and ground effect meshes respectively. There were four template files used for different Fluent setups. The different setups were for with and without blowing and with and without ground effect and all the

permutations. By making both of those decisions, the required template file was determined and would later be sent to the remote computer.

\section{Lines 68-83: Ground Effect Height}

This section asks what ground effect height is required. It has an upper limit of 100 meters and a lower limit of 1.5 meters which can easily be changed if it is so desired.

\section{Lines 87-106: Angle of Attack with Ground Effect}

For normal cases without ground effect, the angle of attack is altered simply by changing the direction of the freestream flow. With a ground in place, that option is not available. The freestream flow should travel parallel to the ground. Therefore, the angle of attack actually needs to be set by rotating the wing. The upper and lower limits were between -10 and 10 degrees.

\section{Lines 110-143: Angle of Attack Name}

As well as sending the correct files to set up the next Fluent case, the MeshSender program also passed along information like the angle of attack and whether or not there was blowing to the Fluent-Setup script so the same question would not need to be asked again. These several lines parse out the given angle of attack and put them into a standard naming format which the grids all used. 


\section{Line 149: Angle of Attack Sign Convention}

Normally a positive sign for angle of attack indicates a pitch up for an aircraft. When the rotation command is performed in Gambit, the positive sign rotated the wing in the opposite direction. This line switched the sign so that the wing would be rotated correctly.

\section{Line 153: Folder1}

There are two different folder names used to organize the ground effect files. Folder1 indicates the main folder for a given flap deflection. For example, if the case with a flap deflection of 42 degrees was required, Folder1 would be 42_flap.

\section{Lines 157-179: Height Name}

To limit the number of digits that would be in a file name, the height in meters was truncated to include only the first two digits. If the height was less than 10 degrees, a leading zero was added. For example, a height of 4.246 meters would show up as 04 .

\section{Line 188: Folder2}

Inside Folder1 (see line 153), a new folder is created for each additional ground effect case generated. Those folders are named in order to indicate the ground effect height (the first two digits in meters), the angle of attack (first two digits, for positive, a negative sign and the first digit for negative), and the flap defletion. For example, for a flap deflection of 42 degrees, a height of 5.723 meters, and an angle of attack of 0 degrees, Folder2 would be G05A00F42. The mesh for these grids uses the exact same naming scheme with ".msh" added to the end of the file. Those meshed are all located in the "G-meshes" directory. 


\section{Lines 192-230: Checking Available Journals and Creating new Ground Effect Grid}

For each flap deflection, a ground effect journal file was required in order to create additional ground effect grids. This journal file gets inserted with the appropriate ground height and then it is moved to the appropriate directory (which is created) to be run by Gambit. Lines 216-219 are where the actual inserting occurs. This process also tells Gambit where to save the files when it is finished with the grid. Line 225 is the actual command to run Gambit with the new ground effect journal file.

\section{Lines 233-242: No Ground Effect}

If the user chooses not to use ground effect, the script skips to here where the mesh folder and template name are then identified for later file transfers.

\section{Lines 247-270: SSH}

With the grid and template files specified, the next step is to transfer them to the remote computer. These lines simply ask for the login name and IP address for the remote computer.

\section{Lines 272-335: Template File Finalization and Zero Blowing}

With all of the design variables finalized, the final templates could be determined as well as the zero blowing grids. This section comes near the end because to create a zero blowing grid for a specific ground height, the blowing grid for that ground height and angle of attack need to exist. After they are created, this section determines whether or not they need to be modified to turn off the blowing. This is where the four permutations come into play 
1. Lines 272-273: Ground Effect With blowing

This may be redundant, but it makes sure that the correct template is sent.

2. Lines 274-303: Ground Effect Without Blowing

These lines specify the correct template file for ground effect without blowing and will also create a Gambit journal file to change the boundary condition of the jet slot to the wall and save that mesh in the appropriate location which is inserted in lines 290 and 291.

3. Lines 304-305: No Ground Effect With Blowing

This is the "normal" case that was run without ground effect and with blowing. That is why this template file is named "template" while the others add " $\mathrm{z}$ " and/or "g" to specify ground effect and zero blowing.

4. Lines 306-335: No Ground Effect Without Blowing

As with the second case (Lines 274-303), this section creates a Gambit journal file to modify a currently available grid so that the jet slot is a wall instead of a mass flow inlet. The files are then saved in the appropriate places which are specified in the journals via lines 324 and 325.

\section{Lines 337-340: Information for Fluent-Setup Script}

If the Fluent-Setup script is run independently of the Mesh-Sender, it will query the user to figure out what grid it is supposed to load and what angle of attack to set the freestream. This information is saved in a text file named "inputs" which is then read by the Fluent-Setup script so that those questions pertaining to that information can be skipped. 


\section{Lines 342-348: Transfer Files}

There are four different files that are transferred to the remote computer for use by the Fluent-Setup script:

1. The mesh file

2. The Fluent-Setup script

3. The appropriate template file

4. The Input file

Each of these files are copied or, in the case of the inputs file, moved to a directory on the Desktop called TF. TF is simply a transfer directory so that only one SCP command would be required to move the entire directory rather than each of the four files individually. This method requires only a single password prompt instead of four. Line 348 removes the TF directory after it has been successfully copied to the remote computer. This copies the TF directory to a directory named .ST which is located in the home directory of the remote computer.

\section{Line 349: SSH and Fluent-Setup Run}

The final line of the script uses SSH to login to the remote computer and runs the Fluent-Setup script. Once the Fluent-Setup script is done running, the prompt returns to the local computer. 


\section{Appendix B - Fluent-Setup-Run Script Documentation}

The purpose of this appendix is to briefly explain the major blocks of code in the Fluent-Setup-Run script. It was written entirely in Bash shell scripting language and can be executed simply by typing "./Fluent-Setup-Run" into the command line with the script in the working directory.

\section{Lines 5-6: Changing the directory}

These lines were actually included for instances when the scrip was run in the home directory instead of the .ST directory. These lines could probably be removed.

\section{Lines 11-34: Reading "inputs"}

If an "inputs" file existed in the .ST directory, these lines would parse out the text in the file and use that information to determine six pieces of information:

1. The particular mesh that was sent from Mesh-Sender

2. The Angle of Attack (if there was ground effect)

3. The Template file to be used

4. The Flap Deflection

5. Whether or not there was to be blowing

6. Whether or not there was ground effect

Each of those six pieces of information were cut out of the "inputs" file to be inserted later into the template along with the other information required to fully define the case file. They are also used to help create the case name.

\section{Lines 35-57: Mach Number}

These lines query the user for the desired Mach number of the freestream flow. For this study, the Mach number was limited to be between 0.05 and less than one. The 
lower end was determined by Fluent's ability to converge on a solution at such low Mach numbers. Even a Mach number of 0.05 was quite unstable. This lower limit may actually be able to be lowered if better grids were used.

\section{Lines 59-75: Uniform Mach Name}

Since each Fluent case file was automatically saved according to the input parameters, it was important that the parameters were all uniform in their naming scheme. This section cuts up the digits for Mach number so that it keeps the first two digits after the decimal place. For example, a given freestream Mach number of 0.15456 would be truncated to 15 .

The format for the entire case name was originally as follows: M\#\#A\#\#M\#\#\#F\#.cas. The decimal points are all removed and the "M", "A", "M", and "F" precede the values for the Mach number, angle of attack, mass flow rate, and flap deflection. The Mach number is the first two digits after the decimal point (Mach 0.4565 $=45)$, the angle of attack contains one digit before and one after the decimal point $(8.456$ degrees $=84)$, the mass flow rate contains two digits before the decimal point and one after $($ Mdot of $4.564 \mathrm{~kg} / \mathrm{s}=045)$, and the flap deflection contains two digits before the decimal place (flap of 6 degrees $=06$ ). A later addition to the script added temperature of the ejection slot as a variable, so it was then added to the case name as well. It falls between the mass flow rate and the flap deflection preceded by a "J" and then listing the first three digits of the temperature $(421.456 \mathrm{~K}=421)$. Ground effect is the last variable to be included and, if it is used, an " $\mathrm{H}$ " followed by two digits of the height off the ground in meters $(8$ meters $=08)$. The current case names are written as M\#\#A\#\#M\#\#\#Ј\#\#F\#\#H\#\#.cas. 


\section{Lines 77-105: Angle of Attack}

The first thing that happens in this block is to check whether or not the angle of attack was sent in the "input" file. If it was not sent, this section queries the user for the angle of attack. It is restricted to be between plus and minus 10 degrees.

\section{Lines 108-141: Naming the Angle of Attack}

As with the other three input parameters, the angle of attack was used in the name of the Fluent case file. The angle of attack was truncated to include one digit before and one after the decimal point with the decimal point removed. For example, an angle of attack of 3.9453 degrees would be truncated to 39 . If it was less than one degree, a leading zero was added to fill the missing digit. An angle of attack of zero would be written as 00 .

\section{Lines 147-150: Converting to Radians}

As with many computational programs, the floating point calculator used in these scripts, BC, works in Radians instead of degrees. These lines convert the angle of attack into radians and then find the components of the angle of attack in the directions of lift and drag. These fractional values are used to set the freestream flow directions and to set the lift and drag forces so they are calculated along the proper vector.

\section{Lines 152-184: Jet Temperature}

This section checks to see if the jet temperature was sent in the "inputs" file and if it was not, proceeds to query the user for the desired jet temperature. It was bounded by 200 and $600 \mathrm{~K}$ for this study. The jet temperature name was defined on line 184 as the first three digits of the temperature $(452.98 \mathrm{~K}=452)$.

\section{Lines 186-280: Mass Flow Rate}


This large block of code allows for two different options in specifying the mass flow rate if it was not already specified by "inputs". Mass flow rate can either be specified in $\mathrm{kg} / \mathrm{s}$ or by the non-dimensionalized parameter $\mathrm{C}_{\mu}$. Since $\mathrm{C}_{\mu}$ is determined by $\mathrm{Eq}(\mathrm{B} 1)$

$$
C_{\mu}=\frac{\dot{m} V_{j e t}}{q_{i n f} S}
$$

Since the mass flow rate and the velocity at the jet are coupled, $\mathrm{C}_{\mu}$ can not be solved for explicitly. A method of bisection technique was used to solve for a mass flow rate that would give the required $\mathrm{C}_{\mu}$. This iterative technique uses a model that relates the velocity at the jet to the mass flow rate as can be seen in $\mathrm{Eq}(\mathrm{B} 2)$.

$$
V_{j e t}=-.1791 \dot{m}^{2}+20.3253 \dot{m}-2.6022
$$

This model was created using a quadratic fit to CFD solutions at different mass flow rates. Obviously there is some error in this model since for zero mass flow there is a negative velocity at the jet. This was found to be acceptable because this iterative solve was meant to provide a $C_{\mu}$ that was close to the one asked for by the user. It was assumed that this code would be used for a study with random placement of the data points so that if the $C_{\mu}$ values moved a small amount than they would still be random. The actual $\mathrm{C}_{\mu}$ value could be calculated when the solution was converged and which could then be used in meta-models. One major restriction for the current model is that the model relating the mass flow rate and the jet velocity has no slot temperature dependence. If the predicted $\mathrm{C}_{\mu}$ is to converge near the final $\mathrm{C}_{\mu}$ value, a new metamodel which is dependent both on mass flow rate and temperature needs to be created and implemented. 


\section{Lines 282-326: Mass Flow Rate Name}

This section takes apart each variation of how the mass flow rate could be entered and puts the digits into the standard form. For example, for a mass flow rate of .2456 $\mathrm{kg} / \mathrm{s}$, the name will be 002 . The mass flow rate in $\mathrm{kg} / \mathrm{s}$ had a range between 0 and 20 for the original study while the $C_{\mu}$ range only varies between 0 and 0.5 . This means that if $\mathrm{C}_{\mu}$ was to be used, the name would only keep one of the significant figures which would be led by two zeros.

\section{Lines 328-416: Flap Deflection}

This section of code defines which flap deflection will be used and therefore which grid is going to be loaded into Fluent. Originally, the script was set up to ask what flap deflection was going to be used and it would load that in. After more experimentation using different grids for the same flap deflection, it became apparent that it might be nice to have the option to load in a grid by its name. Currently, the user has to option to specify the grid by either the flap deflection, if the standard grid is going to be used, or any grid name.

If the grid is specified by flap deflection, a couple procedures are performed. The specified name is converted to the standard name and then there is a search to see if that grid is in the current directory. If it is not, the user will again to prompted to specify the flap deflection. If the second option is used for specifying a grid with any name, the name is modified to make sure that ".msh" is removed in order to be consistent and then the name is stored as usual. Keep in mind that the name of the mesh no longer contains the same number of digits as the standard name since there is no uniform method for shorting an arbitrary name. This functionality was meant as more of a convenience in 
order to test a new grid rather then run an entire study with an inconsistent naming scheme.

\section{Lines 418-601: Ground Effect}

This is the largest of the blocks in the code which performs a number of functions. The two major functions are that the ground height is determined in order to finalize which grid is being used and the template is locked down after this section.

\section{Lines 427-470: Ground Height and Name}

Once ground effect is chosen, a distance to the ground needs to be specified in meters. The script is set up to have an acceptable range between 100 and 1.5 meters. The ground height is then manipulated to be in the consistent form of including the two digits before the decimal point. For example, a height of 8.456 would be cut to 08 .

\section{Lines 450,483-493: Folder Names and Template}

There are two different folder names used to organize the ground effect files. Folder1 indicates the main folder for a given flap deflection. For example, if the case with a flap deflection of 42 degrees was required, Folder1 would be 42 flap.

Inside Folder1 (see line 450), a new folder is created for each additional ground effect case generated. Those folders are named in order to indicate the ground effect height (the first two digits in meters), the angle of attack (first two digits, for positive, a negative sign and the first digit for negative), and the flap deflection. For example, for a flap deflection of 42 degrees, a height of 5.723 meters, and an angle of attack of 0 degrees, Folder2 would be G05A00F42. The mesh for these grids uses the exact same naming scheme with ".msh" added to the end of the file. Those meshed are all located in the "G-meshes" directory. 
At this point of the script, the mass flow is specified, as well as the flap deflection and ground effect height. With that much information, the appropriate template file can be determined as can be seen on lines 484 and 490 .

\section{Lines 495-542: Alternate Name of the Mesh}

As with specifying the flap deflection, this section gives the user an option as to how the mesh is specified. It searches for an available grid with the given flap deflection, angle of attack, and ground effect height. If it is not found, this section of the code gives the user the chance to specify a different name for the mesh if the default method was not used (G\#\#A\#\#F\#\#.msh). If a different name was specified, then the script would search for the new mesh and make sure it was there before running Fluent. Since ground effect was to be specified, it became necessary to modify the boundary condition of the ground. Instead of having the wing move through the fluid as it would in reality, Fluent moves the fluid around the wing. To create the relative velocity of the wing and the ground, the ground needed to become a moving boundary which moved at the same velocity as the freestream flow. Lines 530-532 incorporate this into the journal to be run at Fluent's initialization.

\section{Lines 544-548: Ground Speed}

These are redundant lines which do the same thing as lines 530-532 except for the standard naming scheme rather than an arbitrary one. It could have been written in a more efficient manner where the same calculations only had to be performed in one place in the code, but it was easier simply to repeat it in two places.

\section{Lines 554-577: No Ground Effect}

Since ground effect was never used for a complete study, these lines of code were more frequently used than the previous blocks. The appropriate template file is locked 
down here and the script checks to see if the required mesh is in the local directory. If not, it lets the user know the mesh file is not present and exits out of the script.

\section{Lines 580-601}

If the "inputs" was present and contained all of the appropriate information about ground effect, this section extracted it and parsed it to the correct variable names. Again, the ground speed was set as well as the appropriate template file.

\section{Lines 603-639: Case and Data Name}

After all the work of creating a standardized naming scheme for the different cases, this section finally pulls is all together and creates the file names. Lines 606-611 add the leading letter in front of the truncated variables: M, A, M, J, F, G which precede the Mach number, angle of attack, mass flow rate, jet slot temperature, flap deflection, and ground height (if ground effect was specified). Lines 615-636 gave the different naming schemes for with and without ground effect and also specified the appropriate variables for the freestream flow and the lift and drag forces. The first three lines in each of the two cases (with and without ground effect) simply created the standardized name for the case and data file. The third lines (618 and 628) added -ur to the end of the names which stands for "unresolved". The cases were set to run for a certain number of iterations before solution adaption was used to resolve the boundary layer. Before the adaption occurred, the solution was saved with the -ur case name in case there were any problems during the adaption.

There are two different schemes for directing the freestream flow for with and without ground effect. With ground effect, the angle of attack was modified by actually rotating the wing while the freestream flow continued to flow parallel to the ground. That is why lines 619-624 have the freestream flow variables and lift and drag variables 
purely as zeros and ones instead of components of the angle of attack as can be seen in lines 629-632. In the standard cases without ground effect, angle of attack is obtained by adjusting the freestream flow and the lift and drag force vectors along the components of the angle of attack.

\section{Lines 641-647: Saving the Ground Height and Angle of Attack}

Since the case name simply uses truncated values for the ground height and angle of attack rather than the actual values, the actual values are stored in a text file by the name of "height_\#\#". This information was not available in the Fluent case or data files. If it was not recorded here, the original Gambit .dbs file would need to be reopened and examined to find the actual values.

\section{Lines 656-669: Copying Variables into the Template}

These lines of the code actually incorporate all of the previous lines of code into the preselected template file (which should be in the current directory) which is then renamed with the same naming scheme as the case and data files with a ".jou" at the end. This section actually creates several files in this process. The "sed" requires that you copy each line from the template to a new file name or else a blank text file will be created instead of the template with the inserted variables. To facilitate this process, eleven different journal files are created. It could actually be done with two, bouncing back and forth, but this process allows for better debugging. The error could be traced to one of the particular journal files and therefore the line of code which referenced it. Line 669 gets rid of all of the eleven superfluous journal files so as not to clog up the working directory. 


\section{Lines 671-673: Removing the Inputs File}

Since the inputs file was automatically read into the script instead of querying the user, it was important that old or stray input files not be left behind which would be inserted into future cases. These lines removed the inputs file to eliminate that possible risk. The Fluent-Setup script could still run without the inputs but the user would then need to specify them manually.

\section{Lines 676-695: GUI or no GUI}

One of the capabilities of this script is the ability to run Fluent without the use of the graphical user interface (GUI). This option allows for cases to be run on remote computers without the need to forward the graphics back to the host computer. In addition, if journals created by the Fluent-Setup script were ever to be used on a cluster, it would most likely be required to run command line only in order to reduce the workload of the computers. In order to implement this method, only the "-g" option needed to be added to the end of the Fluent initialization line which is exactly what these lines of code do. The hard work was altering the template files so that all of the Fluent commands were command line based rather than GUI based. The template files became much easier to read and each line of the file was much shorter without all of the GUI commands which resulted in much simpler template files. The command line template files are the default templates used for any journal created by this script. The old GUI based templates exist in the same directory as the command line template files with "gui" leading the respective template name.

\section{Lines 697-714: Which Computer is Fluent Running On}

The particular parallization method which was used differed between the computers in the ATL versus the remote computers that were previously in 41-211 (with 
which almost all the cases were run). In addition, the Fluent directory was located in different locations on the different machines. These lines of the code resolve these issues and then initialize Fluent with the newly created journal file which will set up and run the previously defined case. 


\section{Appendix C - Example Template File}

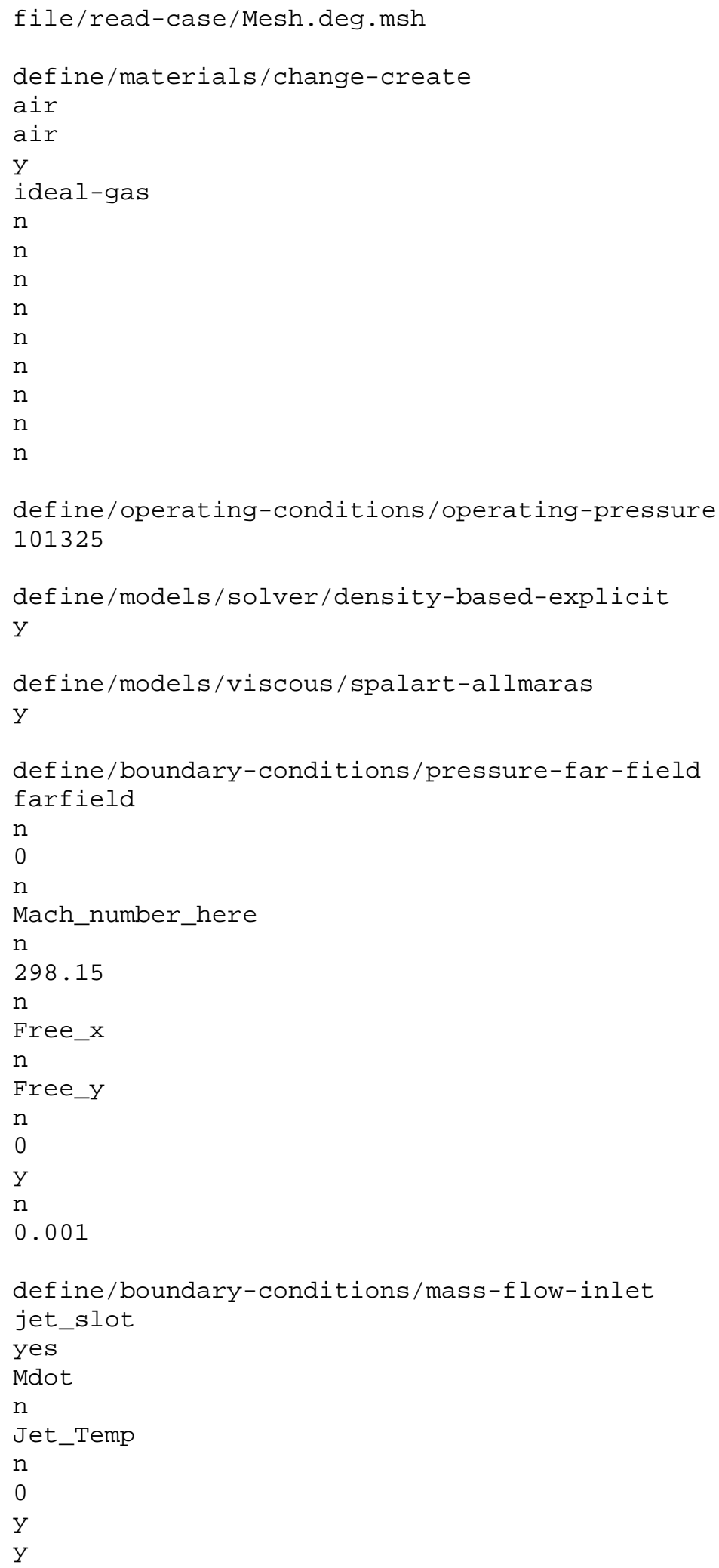




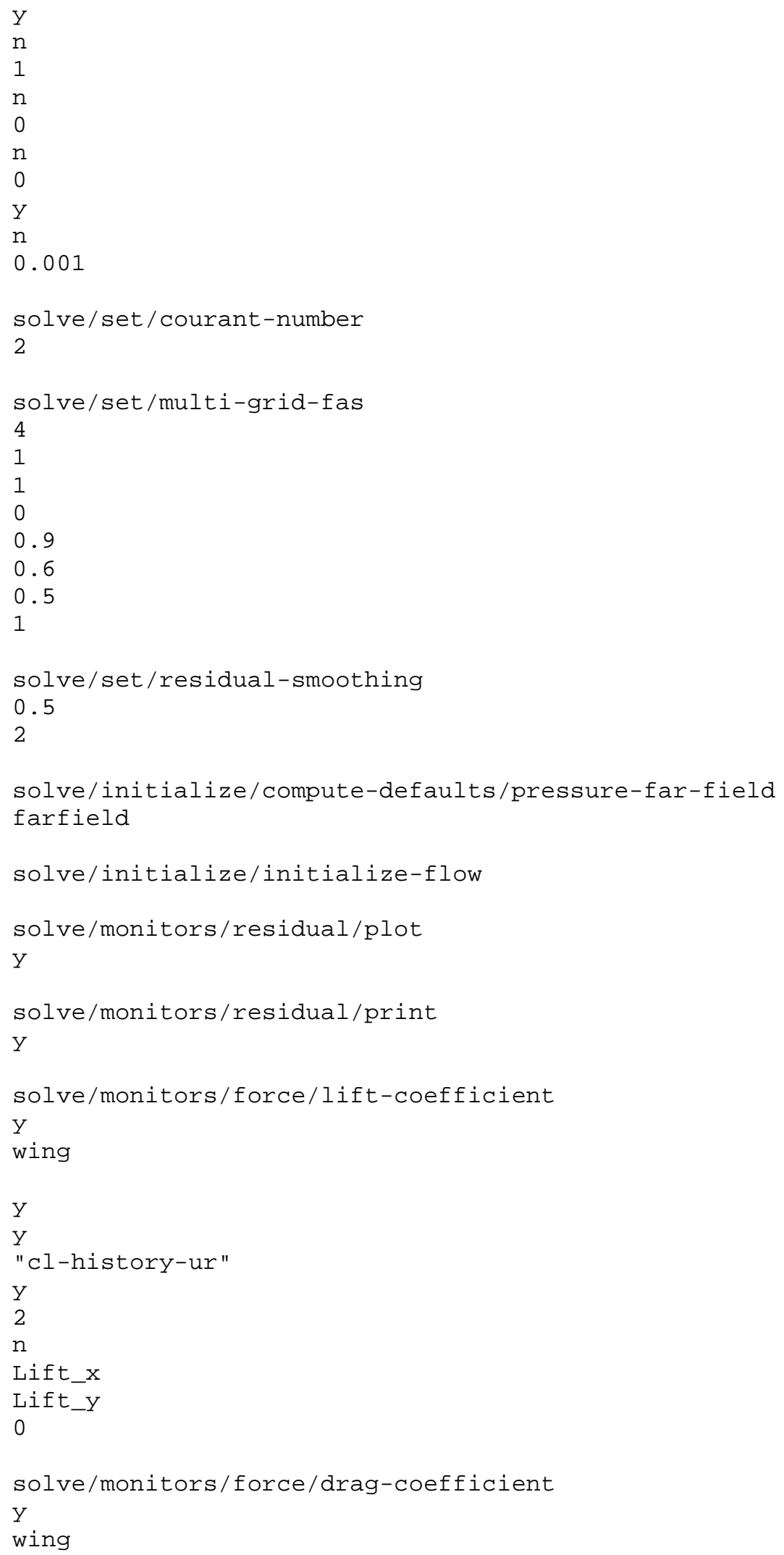




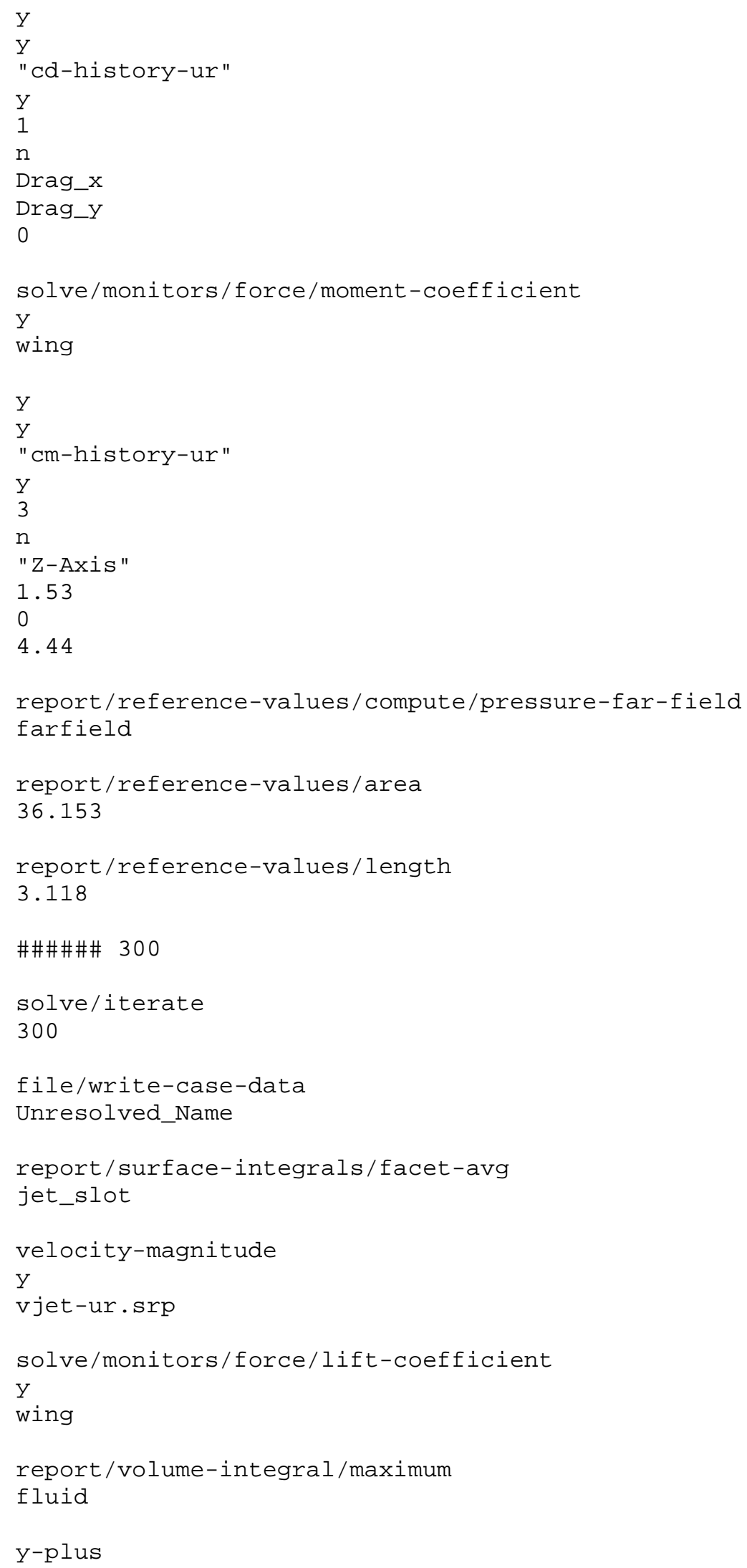




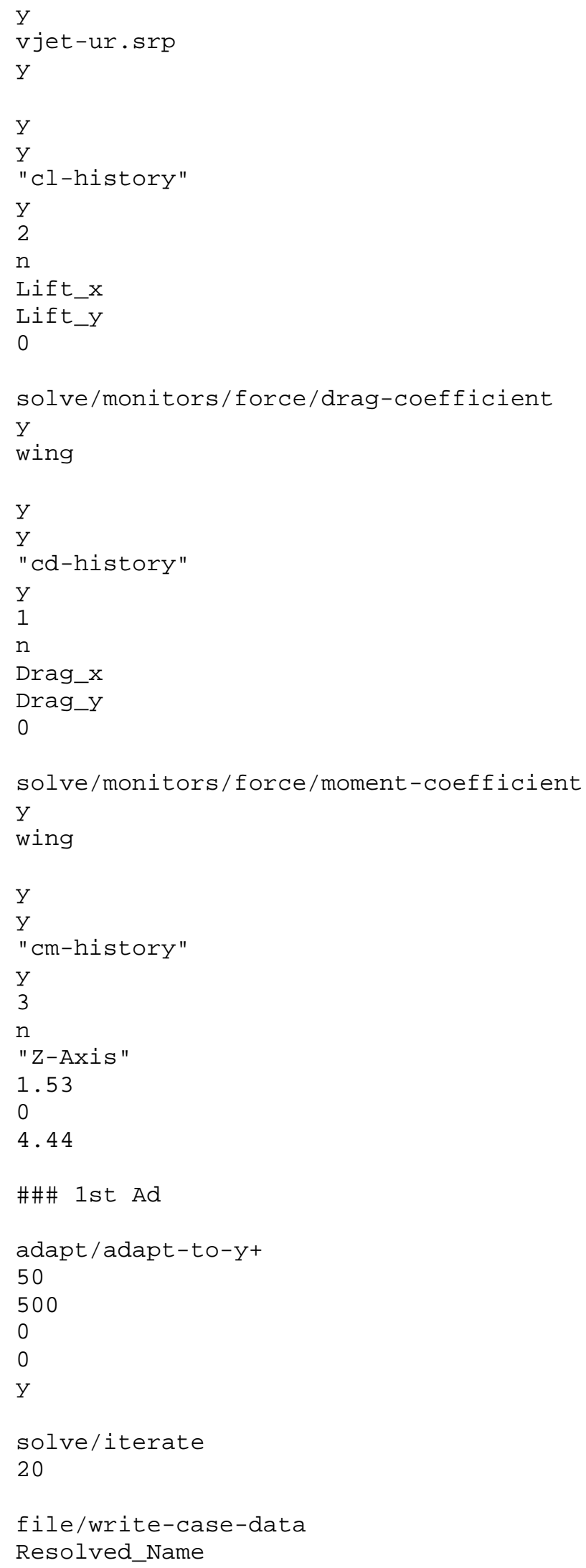




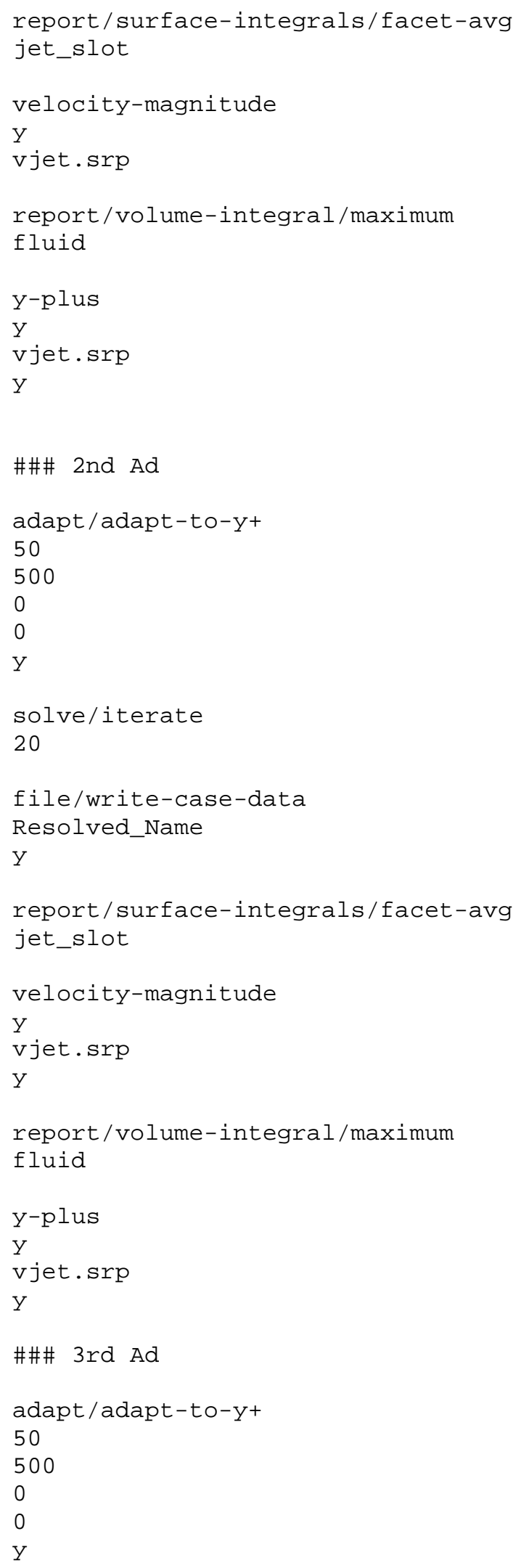




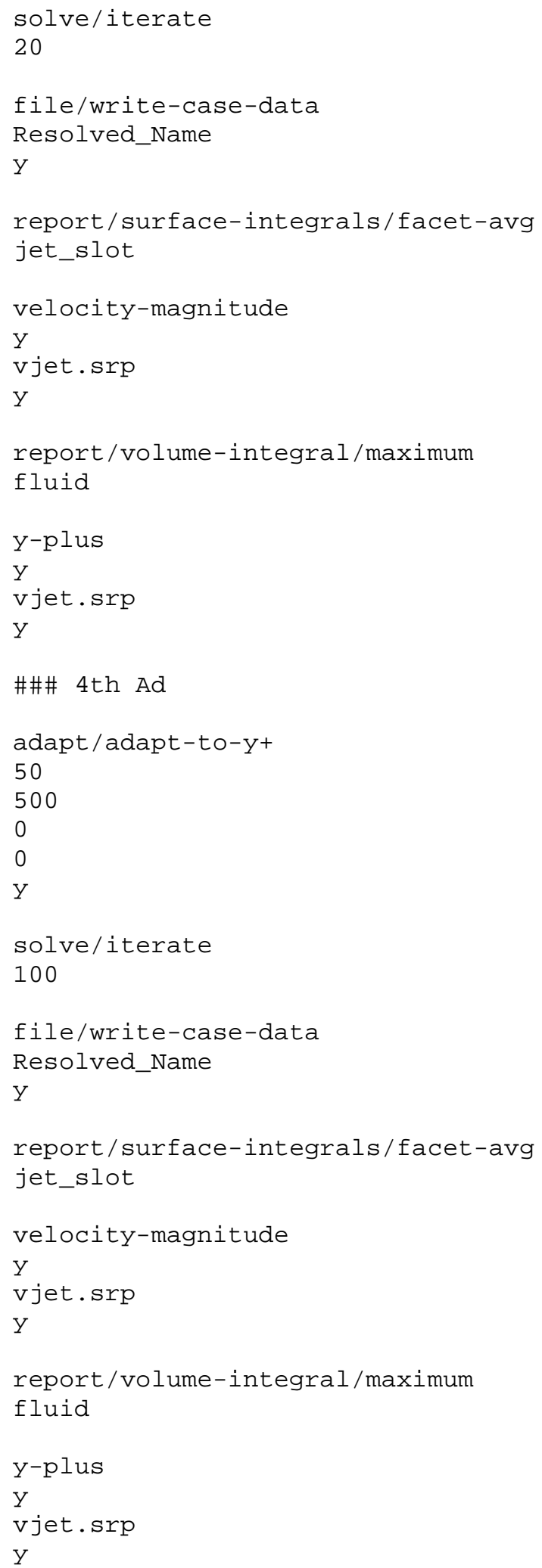




\section{Appendix D - Modified Rotation Calculations}

A way around the problem of how to determine the rotation velocity is to redefine the rotation velocity based on the actual lift produced throughout the takeoff run and the weight of the aircraft. During takeoff there should be some point when the lift reaches some percentage of the weight and rotation can be achieved. If the rotation speed can be defined by this metric it would be independent of the $\mathrm{C}_{\mathrm{L}}$ and could be applied to any aircraft rather than only traditional designs. At rotation, the lift over weight will be some percentage, constant $\mathrm{k}$, of the lift over weight at stall, which is 1 as seen in $\mathrm{Eq}(\mathrm{D} 1)$.

$$
\frac{L_{\text {stall }}}{W}=k * \frac{L_{r}}{W}
$$

Now, if some common assumptions are used that relate the max lift coefficient to the rotation lift coefficient and the stall velocity to the lift velocity, one can reduce Eq(D1) using the following logic.

Simplifying $\operatorname{Eq}(\mathrm{D} 1)$ yields:

$$
\begin{gathered}
L_{\text {stall }}=k * L_{r} \\
V_{\text {stall }}^{2} * C_{L_{\max }}=V_{r}^{2} * C_{L_{r}} * k
\end{gathered}
$$

Below are the equations for the assumptions:

$$
V_{r}=C * V_{\text {stall }} \approx 1.05 \mathrm{~V}_{\text {stall }} \quad \frac{C_{L_{r}}}{C_{L_{\max }}}=0.9
$$

Substituting back into Eq(D1): 


$$
\begin{aligned}
& \frac{V_{\text {stall }}^{2}}{0.9}=\left(1.05 V_{\text {stall }}\right)^{2} * k \rightarrow k=1.223 \\
& 1=\frac{1.223 * L_{r}}{W} \\
& \frac{L_{r}}{W}=0.817
\end{aligned}
$$

This yields a criterion for when rotation occurs that is not dependent on the actual $C_{\text {Lmax }}$ of the aircraft only the ratio of $\mathrm{C}_{\text {Lrotation }}$ and $\mathrm{C}_{\mathrm{Lmax}}$. This follows the same basic format as the other preliminary design guidelines stating that the rotation velocity is a percentage of the stall velocity. This makes it easy to define a rotation speed in a number of different cases using the same flavor of regulations.

The downside for this is the need to have $\mathrm{C}_{\mathrm{L}}$ data at each velocity along a ground trajectory in order to figure out where the lift to weight ratio reaches 0.817 . However, this is not particularly constraining because it works with a constant CL model as well as more complicated aerodynamics models. All that is needed is to run a series of test cases and then find the velocity that matches the ratio. Optimization processes could be used if desired but are by no means required. This also works well with an integration of the equation of motions into a trajectory model since all of the required data would be available after the integration. 